\title{
The Dynamics of Personality and Identity in
}

\author{
Adolescence
}

Theo Klimstra 


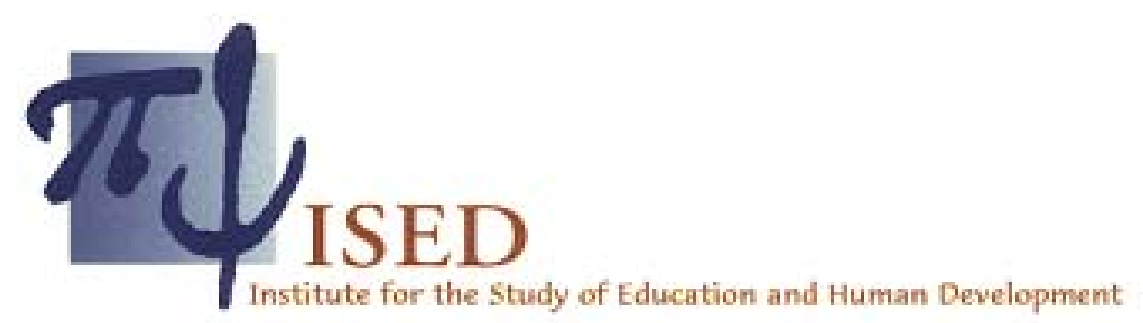

ISBN: 978-90-393-5333-2

Printed by Ipskamp Drukkers, Enschede, The Netherlands

\section{(C) Theo Klimstra}

All rights reserved. No part of this publication may be reproduced, stored in a retrieval system, or transmitted in any form or by any means, mechanically, by photocopy, by recording, or otherwise, without permission from the author. 


\title{
The Dynamics of Personality and Identity in Adolescence
}

Persoonlijkheid en Identiteit in de Adolescentie

(met een samenvatting in het Nederlands)

\begin{abstract}
Proefschrift
ter verkrijging van de graad van doctor aan de Universiteit Utrecht op gezag van de rector magnificus, prof. dr. J. C. Stoof, ingevolge het besluit van het college voor promoties in het openbaar te verdedigen op dinsdag 25 mei 2010 des middags te 2.30 uur

door
\end{abstract}

Theo Albert Klimstra

geboren op 8 juli 1982, te Harlingen 


\section{Promotor:}

Prof. dr. W. H. J. Meeus

\section{Co-promotoren:}

Dr. W. W. Hale III

Dr. Q. A. W. Raaijmakers 


\section{Leden Beoordelingscommissie}

Prof. dr. T. F. M. ter Bogt

Dr. H. A. Bosma

Prof. dr. M. Deković

Prof. dr. L. Goossens

Prof. dr. M. J. A. M. Verkuyten
Universiteit Utrecht

Rijksuniversiteit Groningen

Universiteit Utrecht

Katholieke Universiteit Leuven

Universiteit Utrecht 

Foar Heit en Pake 



\section{Contents}

Chapter $1 \quad$ General Introduction 11

Chapter 2 Maturation of Personality in Adolescence 29

Chapter 3 Longitudinal Associations Between Personality Traits and Problem 63

Behavior Symptoms in Adolescence

Chapter 4 A Developmental Typology of Adolescent Personality 91

Chapter $5 \quad$ Longitudinal Associations Between Personality Profile Stability and 111

Adjustment in College Students: Distinguishing Among Overall Stability,

Distinctive Stability, and Within-Time Normativeness

Chapter 6 Too Early or Too Late: Hypermaturity and Immaturity of Personality

in Adolescent Boys and Girls

Chapter 7 Identity Formation in Adolescence: Change or Stability?

Chapter 8 Short Term Fluctuations in Identity: Introducing a Micro-level

Approach to Identity Formation

Chapter 9 Identity Formation in Juvenile Delinquents, Clinically Referred Youth, 201 and Adolescents from the General Population

Chapter $10 \quad$ General Discussion 219

$\begin{array}{ll}\text { References } & 245\end{array}$

$\begin{array}{ll}\text { Summary } & 261\end{array}$

Samenvatting (Summary in Dutch) 263

Dankwoord (Acknowledgements in Dutch) 265

$\begin{array}{ll}\text { Curriculum Vitae } & 267\end{array}$

$\begin{array}{ll}\text { Publication List } & 269\end{array}$ 

Chapter 1

General Introduction ${ }^{1}$

${ }^{1}$ Portions of this chapter will be published as: Klimstra, T. A., Hale, W. W., Raaijmakers, Q. A. W., Branje, S. J. T., \& Meeus, W. H. J. (in press). Personality assessment and maturation. In R. J. R. Levesque (Ed.), Encyclopedia of adolescence. Heidelberg, Germany: SpringerVerlag. 


\section{Chapter 1. General introduction}

When persons describe themselves, they are typically referring to certain characteristics, such as "outgoing", "nervous", "messy", “friendly" and "creative”. Exactly these kinds of characteristics define one's personality. In personality psychology, which focuses on individual differences in the just-mentioned characteristics, there has been a longstanding debate on what would be the most appropriate set of characteristics to describe people by. In the last twenty years, there has been a growing consensus on this issue, as a majority of researchers now agree that personality characteristics can be subsumed in five broad traits: the Big Five (Caspi, Roberts, \& Shiner, 2005). These five broad factors are Extraversion, Agreeableness, Conscientiousness, Emotional Stability, and Openness to Experience (McCrae $\&$ Costa, 1987).

Extraversion (as opposed to introversion) refers to being dominant, outgoing and energetic in interpersonal situations. Extraverted individuals tend to enjoy social attention and experience frequent positive moods. Agreeableness encompasses a wide range of characteristics that are all indicative of an individual's competence and willingness to maintain positive and reciprocal relationships with others; in other words, pro-social behavioral tendencies. Conscientiousness refers to personal characteristics indicative of behavioral and cognitive control. Conscientious individuals are neat, orderly, and responsible persons, who have little trouble with dividing their attention in an optimal way. Emotional Stability (as opposed to Neuroticism) is indicative of a person's ability to deal with negative emotions in an effective manner. A person with low levels of Emotional Stability is often insecure, has a tendency to experience negative moods, gets frustrated easily, and is commonly anxious. Openness to Experience refers to a person's style of dealing with new information and opportunities. It comprises aspects such as creativity, imagination, originality, and curiosity.

Another aspect of the self that becomes more salient in adolescence is identity (Erikson, 1950, 1968). Identity has been conceptualized as "self-sameness and continuity to... the style of one's individuality, and that this style coincides with the sameness and continuity of one's meaning for significant others in the immediate community" (Erikson, 1968, p. 50). Although Erikson's $(1950,1968)$ initial work on identity sparked an interest in research on the topic, it could not be directly translated into an empirically testable construct. To overcome this problem, Marcia (1966) elaborated on Erikson's work and developed the identity status paradigm. In this paradigm, the focus is on two key processes of identity 
formation: exploration of several life choices in relevant developmental domains, and commitment, signifying the degree to which one has made a choice and identifies with that choice in a certain developmental domain. Based on processes of exploration and commitment, individuals can be classified in either of four distinguishable identity status: Diffusion (little exploration and little commitment), Foreclosure (strong commitment, but little exploration), Moratorium (active exploration, but a lack commitment), and Achievement (strong commitment, after a period of extensive exploration).

In the current dissertation, the focus will be on these two central features of an individuals self-concept: "who am I as a person" (personality), and "can I identify myself with certain aspects of the life I am currently living" (identity formation). First, several approaches to personality development will be discussed. Second, we will move to a discussion of contemporary approaches to identity formation.

\subsection{Development of Personality from Adolescence through Early Adulthood}

Adolescence is the period in life in which individuals gradually change from a child to an adult (Erikson, 1950). Along with physical changes (e.g., Petersen, Crockett, Richards, \& Boxer, 1988) and neurocognitive changes (e.g., Giedd et al., 1999), role expectations also change substantively. In this context, adolescents are expected to perform school work in an increasingly independent manner, form interdependent (hence more mature) friendships (De Goede, Branje, Delsing, \& Meeus, 2009; Furman \& Buhrmester, 1992), explore romantic relations, and encounter their first occupational experiences (e.g., becoming a paperboy/girl or working during the weekends in the local supermarket). Taking up such tasks brings increased social expectations, as individuals are expected to behave in an increasingly more mature manner. For example, when taking on a job, one is expected to show up on time and work in a structured manner. Characteristics related to these capacities are subsumed under the trait of Conscientiousness. The capability to maintain reciprocal relationships, even with people that one does not necessarily like (a capability subsumed under the trait of Agreeableness) is also important when getting a job, as one cannot choose one's colleagues. Apart from that, the capability of maintaining reciprocal relationships is, of course, also important for establishing friendships and romantic relations. Several other characteristics are also important for adolescents, such as dealing with setbacks (indicated by Emotional Stability), and convincing others of ones capability by attracting their attention (a capability indicated by Extraversion). These are only a few examples, but one can imagine that the aforementioned capabilities are 
useful in many contexts. Thus, as social expectations increase, it is likely, or it would at least be beneficial, for an adolescents' personality to change accordingly. In other words: adolescents are expected to exhibit maturation of personality.

To assess personality maturation, one can either pursue variable-centered approaches (i.e., focusing on how particular traits change on average in a group of individuals, and whether individual differences in traits are stable or fluctuate across time), or person-centered approaches focusing on how a configuration of traits is organized within an individual person. Both kind of approaches provide unique information about how the nature of personality changes across time (e.g., Block, 1971). We will now discuss the specific variable-centered and person-centered approaches to research on personality that will be employed in the various studies of this dissertation.

\subsubsection{Variable-Centered Approaches to Personality}

Two variable-centered approaches to change and stability in personality will be employed in this dissertation. The first one, mean-level change, is quite obvious. Mean-levels of personality traits can change in a population. For example, adolescents can on average become more agreeable with age. Mean-level change is typically assessed with techniques such as Repeated Measures Analysis of Variance, or Latent Growth Models (e.g., Duncan, Duncan, Stryker, Li, \& Alpert, 1999).

Second, there is rank-order stability, which indicates to what extent inter-individual differences on personality traits remain stable across time. Regardless of whether the average levels of personality traits change, these inter-individual differences can be stable or can fluctuate over time (e.g., Roberts, Walton, \& Viechtbauer, 2006; Roberts \& DelVecchio, 2000). This makes it important to consider rank-order stability alongside mean-level change. If one is interested in increases of rank-order stability across time (i.e., whether interindividual differences become more set when adolescents grow older), one needs to employ ordinary pearson test-retest correlations. It is, however, also possible to predict the rank-order of individuals on a certain variable at a particular point in time (e.g., depression at time 2) from the rank-order of these same individuals on another variable at a previous point in time (e.g., extraversion at time 1). In that case, a cross-lagged panel model (Burkholder \& Harlow, 2003 ) is the most appropriate type of analysis. 


\subsubsection{Person-Centered Approaches to Personality}

It has been argued that variable-centered approaches do not capture the true nature of personality, because they provide little information as to how a configuration of traits is organized within an individual person (e.g., Allport, 1954; Robins, John, Caspi, Moffitt, \& Stouthamer-Loeber, 1996). To investigate the true nature of personality, person-centered approaches with a focus on stability and change of a configuration of multiple personality traits within a single person are needed.

One of these person-centered approaches to change and stability of personality is profile stability. Profile stability provides information on the stability of a constellation of traits for every single person in a research sample. To assess profile stability, the intraindividual consistency of the mean scores on personality traits is computed. It is possible, for example, that a person is more agreeable than conscientious, more conscientious than emotionally stable, more emotionally stable than extraverted, and more extraverted than open to experience. The degree to which this pattern of traits within a person remains the same across time indicates that person's profile stability (e.g., Furr, 2008; Klimstra, Luyckx, Hale, Goossens, \& Meeus, in press; Roberts, Caspi, \& Moffitt, 2001). Thus, profile stability indicates the extent to which, for example, the outgoing and friendly, but disorganized person is still like that one year later.

A general and empirically supported assertion with regard to profile stability is that it is indicative of psychological health, with individuals with a more stable profile displaying less internalizing and externalizing problem behaviors (e.g., Donnellan, Conger, \& Burzette, 2007; Furr, 2008; Klimstra, Luyckx et al., in press; Ozer \& Gjerde, 1989; Roberts et al., 2001). However, Furr (2008) asserted that it may not be the stability of a personality profile that is associated with psychological health. Furr (2008) explained this as follows: within a population mean-levels of Big Five traits might be quite stable across two subsequent measurement occasions. In such a case, the norm personality profile (i.e., a profile based on mean-level scores on all Big Five traits) is quite stable across time. When an individual has a personality profile that matches the norm profile at both measurement occasions, he or she would reflect high profile stability. However, in that case it is not clear what drives the associations with psychological health: (a) the degree to which an individual's personality profile is similar to the norm profile or (b) the stability of this individual's personality profile. Because of this, Furr (2008) asserted that researchers should correct for normativeness of personality profiles (i.e., the degree to which an individual's personality profile matches the norm profile within a population) when examining the effect of personality profile stability. 
For that purpose, Furr (2008) advocated a distinction between overall stability (profile stability uncorrected for normativeness), distinctive stability (profile stability corrected for normativeness) and normativeness of personality profiles. Overall stability, distinctive stability, and normativeness can be calculated with $q$-correlations.

Overall stability, distinctive stability, and normativeness are not the most commonly used person-centered approach to personality. By far the most prominent person-centered approach to adolescent personality is Block and Block's (1980) typological approach. They distinguished three replicable personality types: Resilients, Undercontrollers, and Overcontrollers, based on the amount of ego-control and ego-resiliency. Ego-control is indicative of a person's ability to deal with his or her impulses, whereas ego-resiliency signifies an individual's ability to modify levels of ego-control as a function of changing environmental demands. Resilients have high levels of ego-resiliency, and are therefore able to flexibly adjust levels of ego-control to changing environmental and situational demands. Both Overcontrollers and Undercontrollers have low levels of ego-resiliency, which causes them to have relatively stable levels of ego-control. In Overcontrollers, levels of ego-control are excessively high, which means that Overcontrollers contain their impulses, and display high levels of inhibition with regard to action and affect. Undercontrollers have excessively low levels of ego-control, resulting in an inability to delay gratification, and a tendency to respond immediately and impulsively to environmental triggers (Block \& Block, 1980).

Because Robins et al. (1996) demonstrated that Resilients, Undercontrollers, and Overcontrollers have clearly distinguishable Big Five profiles, several subsequent studies (e.g., Akse, Hale, Engels, Raaijmakers, \& Meeus, 2004; Asendorpf, Borkenau, Ostendorpf, \& Van Aken, 2001; Dubas, Gerris, Janssens, \& Vermulst, 2002) extracted personality types directly from Big Five data. For this purpose, they applied cluster analysis to Big Five ratings (see for example Akse, Hale, Engels, Raaijmakers, \& Meeus, 2007, for an explanation of this technique). However, these studies all constructed personality types using data obtained on one point in time. As such, they constructed cross-sectional personality types. Originally, Block (1971) made a case for the study of types of personality development (i.e., types based on mean-levels and changes in personality traits) instead of just types of personality (i.e., types solely based on mean-levels of personality traits). Such developmental personality types can be identified with Latent Class Growth Analysis (LCGA; Nagin, 1999, 2005). 


\subsection{Identity Formation in Adolescence: a Dynamic Approach}

After Marcia (1966) provided an empirical elaboration on Erikson's (1950; 1968) theoretical writings on the concept of identity, Marcia's identity status approach has dominated research on identity formation for several decades. However, the preoccupation of this approach to the classification of individuals into statuses, and its inability to explain changes in identity after an identity achievement status has been reached, led to critical comments by several authors (Bosma, 1985; Côté \& Levine, 1988; Grotevant, 1987) and Marcia himself (Marcia, 1993). This critique triggered the development of new models of identity formation (Meeus, 1996; Luyckx, Goossens, Soenens, \& Beyers, 2006), which emphasize that identity formation is a dynamic process. A key assumption of these models is that there is no endpoint in identity formation, as commitments will, to some extent, always be re-evaluated. One of the most recent of these models, the three-dimension model developed by Meeus (Crocetti, Rubini, \& Meeus, 2008), will be employed in the current dissertation.

In the model by Crocetti, Rubini, and Meeus (2008), three key processes in identity formation are distinguished: commitment, reconsideration, and in-depth exploration. Commitment is similar to the original definition by Marcia (1966), and refers to being committed to one's choice of identity and engaging in relevant activities towards the implementation of that choice. However, instead of Marcia, who only examined one type of exploration, two types of exploration are distinguished in the three-dimension model: in-depth exploration and reconsideration of commitment. In-depth exploration represents the extent to which adolescents explore current commitments actively, reflect on their choices, and search for additional information about these choices. It refers to adolescents exploring the merits of their current commitments, without questioning these commitments. Reconsideration of commitment refers to adolescents comparing their present commitments with possible alternatives. When reconsideration occurs, it has a short-term detrimental effect on adolescents, as it reflects uncertainty about commitments. Therefore, reconsideration is positively related to problem behaviors, such as depression and delinquency (Crocetti, Rubini, \& Meeus, 2008). However, when the developmental context of an individual changes, it can be necessary to re-evaluate or even replace old commitments. Thus, the long-term effects may, in fact, be positive since adapting one's commitments can be necessary when current commitments are no longer suitable in a new context.

Recent theoretical work by Lichtwarck-Aschoff, Van Geert, Bosma, and Kunnen (2008) pointed out that it is important to consider time-scale when examining identity formation. 
More specifically, they proposed that it is important to distinguish among the short-term (i.e., micro) and long-term (i.e., macro) dynamics of identity formation. For example, one could imagine that it matters whether adolescents report on their sense of commitment across the last day (i.e., micro-level commitment) or on their sense of commitment in general (i.e., macro-level commitment). In the current dissertation, we will not only assess identity formation at a macro-level, but we will also explore how the micro-level relates to the macrolevel.

\subsection{Research Questions Personality Development}

\subsubsection{How do adolescent's personalities develop?}

In chapter 2 we will explore the dynamics of adolescent personality development. Following previous work (e.g., Roberts et al., 2001), several aspects of personality will be distinguished: (a) mean-level change, (b) rank-order stability, and (c) profile stability. Adolescence is generally perceived as a period of maturation, and the purpose of chapter 2 was therefore to examine whether an adolescent personality also matures. Personality maturation would be reflected in increasing mean-levels, inter-individual differences becoming more set (i.e., increases in rank-order stability), and increasingly more stable personality profiles. Because girls tend to mature earlier then boys, we will also explore gender differences in all indicators of change and stability.

\subsubsection{How does the developing personality relate to the development of problem behavior} symptoms?

Numerous studies have found that personality traits and problem behavior symptoms tend to be related to one another. The direction of effect between personality and problem behavior is, however, unclear. With regard to these effects, there are two hypotheses: (a) the scar hypothesis, proposing that problem behavior symptoms affect one's personality traits, and (b) the vulnerability hypothesis, which states that certain personality characteristics are risk factors for the development of problem behavior. To determine the direction of effects between personality and problem behavior, we will simultaneously test the scar and the vulnerability hypotheses in Chapter 3. For that purpose, we will use longitudinal cross-lagged panel models. 


\subsubsection{Are traditional personality types replicable as trajectories of personality development?}

Since Robins et al. (1996) demonstrated that Block and Block's (1980) personality types (i.e., Resilients, Undercontrollers, and Overcontrollers) have specific Big Five profiles, a substantive amount of studies on these types have emerged. However, Block (1971) originally made a case for types of personality development instead of types of personality in order to examine how these types manifest themselves at different points in time. That is, Block advocated the incorporation of normative developmental trend in the classification of individuals into types. Block distinguished several of these longitudinal types, but since the publication of this seminal work in his book "Lives Through Time", several methodological improvements have been made. As such, we will revive Block's search for a developmental typology of personality with state-of-the-art methodology (Latent Class Growth Analysis; Nagin, 1999, 2005) in Chapter 4. To validate the personality types, we will examine their associations with several adjustment measures.

\subsubsection{How do different aspects of personality profile stability relate to adjustment?}

Throughout the years, several studies have shown that personality profile stability is positively related to adjustment (Donnelan et al., 2007; Ozer \& Gjerde, 1989; Roberts et al., 2001). Recently, Furr (2008) proposed that profile stability (which he referred to as overall stability), should be subdivided into normativeness (i.e., the degree to which an individual's personality profile matches the average personality profile in a population) and distinctive stability (i.e., overall profile stability adjusted for normativeness). Moreover, Furr hypothesized that it would not be the stability of a profile (i.e., distinctive stability), but the degree to which a person's profile matches the average profile in a population (i.e., normativeness) that would be related to adjustment. We will test this assertion in Chapter 5, using longitudinal path models in which several adjustment measures (i.e., depression, selfesteem, and delinquency) will be related to three aspects of profile stability (i.e., overall stability, distinctive stability, and normativeness).

\subsubsection{What are the correlates of off-time personality development?}

It has previously been demonstrated that off-time pubertal development has a detrimental effect on adolescent psychosocial adjustment. To our knowledge, there are, remarkably, no studies on off-time personality development. As such, we will explore the correlates of offtime personality development in Chapter 6. 
Because personality refers to a patterning of a set of traits within a single-person (Allport, 1954), multiple traits (i.e., all Big Five traits) need to be considered at the same time when examining off-time personality development. Furr (2008) recently suggested a way to calculate hypermaturity (i.e., being ahead) and immaturity (i.e., lagging behind) in personality development. Hypermaturity would be evident when a young individual (e.g., a 12-year-old) would have a personality profile that correlated strongly with the normative personality profile of an older individual (e.g., the profile of the average 20-year-old), and immaturity would be indicated by an older individual (e.g., a 20 -year-old) having a personality profile strongly correlating with the normative profile of a younger individual (e.g., the profile of the average 12-year-old). Associations between profiles are calculated with $q$-correlations for every single person in a dataset. $Q$-correlations therefore have a mean and a standard deviation and can be treated in the same way as one would treat scores on, for example, a depression scale. As such, we will correlate $q$-correlations representing hypermaturity and immaturity of personality with several adjustment measures (i.e., anxiety, depression, delinquency, aggression, and conflict with parents) to examine the correlates of off-time personality development. In addition, we will examine whether the possible associations between off-time personality development and problem behavior hold across time by examining development of the mentioned indicators of adjustment in adolescents that reflect different levels of hypermaturity and immaturity. All analyses will be run for boys and girls separately, because of anticipated gender differences in personality development.

\subsection{Research Questions Identity Formation}

\subsubsection{What is the course of adolescent identity formation?}

Identity formation is perceived to be a central developmental task of adolescence (Erikson, 1950). There have been quite a few studies examining the dynamics of identity formation in adolescence, but only a few have employed longitudinal data to examine changes in commitment and exploration processes. To our knowledge, there have been no studies examining changes in these processes across the entire period from early to late adolescence. Therefore, we will explore the change and stability in identity formation from early to late adolescence in Chapter 7.

Following previous work on personality development (e.g., Roberts et al., 2001), we will follow the same strategy as we did in Chapter 2 by distinguishing among several aspects of change and stability: (a) mean-level change, (b) rank-order stability, and (c) profile 
stability. Theoretically, identity formation should be characterized by increases in commitment, decreases in reconsideration, and increases in in-depth exploration. Moreover, inter-individual differences in these identity dimensions, and identity profiles should become more stable as adolescents grow older. Because girls tend to mature earlier then boys in many respects, we will also examine whether girls are also ahead on boys with regard to identity formation.

\subsubsection{How do the short-term dynamics of identity formation operate?}

Change and stability in identity formation have to some extent been examined at the macro level (i.e., considering changes in a general sense of commitment and exploration), whereas there has been very little work focusing on the micro level of identity formation (i.e., considering changes in one's sense of commitment and exploration on a weekly or even daily basis). In Chapter 8, we will explore the short-term dynamics (i.e., micro level) of identity formation. First, we will explore whether identity formation can be measured in a reliable and valid manner with single-item measures. Second, reciprocal day-to-day effects between commitment and reconsideration will be examined. Third, the impact of day-to-day fluctuations in commitment and reconsideration on subsequent levels of these two identity dimensions will be assessed. A domain-specific approach to identity formation will be adopted, as we will distinguish among an ideological domain (i.e., educational identity) and an interpersonal domain (i.e., relational identity with regard to friendships).

\subsubsection{What are the differences and similarities between identity formation in juvenile} delinquents, clinically referred youth, and adolescents drawn from the general population? Numerous studies have explored the links between identity formation and problem behavior symptoms in samples representative of the general adolescent population. It is, however, unclear to what extent the findings obtained in these studies are indicative of differences in identity formation between youth drawn from the general population, and youth displaying severe problem behavior. Therefore, Chapter 9 provides an attempt to compare identity formation among juvenile delinquents, clinically referred youth, and adolescents drawn from the general population. 


\subsection{Study Designs and Measures}

In the current dissertation, we employed data from three longitudinal studies: CONflict And Management Of Relationships (CONAMORE; Meeus et al., 2006, chapter 2, 3, 4, 6, and 7), Research on Adolescent Development And Relationships (RADAR, e.g., Eichelsheim et al., 2009, chapter 8), and Leuven - Trajetories of Identity DEvelopment Study (L-TIDES, Luyckx, Goossens, \& Soenens, 2006). In addition, we used one cross-sectional dataset involving juvenile delinquents and clinically referred youth (Klimstra, Crocetti, Hale, Kolman, Fortanier, \& Meeus, 2009).

\subsubsection{CONAMORE Samples and Design}

CONAMORE is an ongoing large-scale longitudinal study tracking psychosocial development in Dutch adolescents. The main focus of the study is on the development of the self (i.e., personality and identity), problem behavior, and relationships with parents and peers. For this purpose, 1313 adolescents have already been followed during five annual measurement waves, with follow-up measurement waves in early adulthood currently underway. The 1313 adolescents are subdivided into an early to middle adolescent cohort $(n=$ $923 ; 12.4$ years of age on average at time $1, S D=.6 ; 50.7 \%$ boys), and a middle to late adolescent cohort ( $n=390 ; 16.7$ years of age on average at time $1 ; S D=.8 ; 43.3 \%$ boys). With regard to ethnicity, $83.4 \%$ of the early to middle adolescents identified themselves as Dutch and 16.6\% indicated that they belonged to ethnic minorities (e.g., Surinamese, Antillean, Moroccan, Turkish). In the middle to late adolescent cohort these figures were $87.4 \%$, and $12.6 \%$, respectively.

\subsubsection{CONAMORE Measures}

Personality. Personality was assessed with the shortened Dutch version of Goldberg's Big Five questionnaire (Gerris et al., 1998; Goldberg, 1992). In this instrument, a 7-point likert scale, with a response format ranging from 1 (completely untrue) to 7 (completely true), is used to assess five personality dimensions: Extraversion, Agreeableness, Conscientiousness, Emotional Stability, and Openness to Experience. All dimensions are measured with 6 items each, such as: 'talkative' (Extraversion), 'sympathetic' (Agreeableness), 'systematic' (Conscientiousness), 'worried' (Emotional Stability, inversely scored), and 'creative' (Openness to Experience). 
Identity Formation. Identity formation was assessed with the Utrecht-Management of Identity Commitments Scale (U-MICS), a self-report measure designed by Meeus (Crocetti, Rubini, \& Meeus, 2008) based on the U-GIDS (Meeus, 1996). With this instrument, 5-point Likert-scale items, with a response format ranging from 1 (completely untrue) to 5 (completely true), are used to assess three identity dimensions: commitment (five items), in-depth exploration (five items), and reconsideration (three items). The same items can be filled out to assess identity dimensions in ideological domains (i.e., education) and interpersonal domains (i.e., friendships). Sample items are: 'My education/best friend gives me certainty in life' (ideological/interpersonal commitment), 'I think a lot about my education/best friend' (ideological/interpersonal in-depth exploration), 'I often think it would be better to try and find different education/a different best friend' (ideological/interpersonal reconsideration). Although U-MICS allows for identity dimensions to be measured in different content domains, we focused on identity dimensions at a global level in chapters 7 and 9. For this purpose we followed the scale construction procedure described by Crocetti, Rubini, and Meeus (2008). A detailed description of the validity of U-MICS is provided by Crocetti, Rubini, and Meeus (2008), and Crocetti, Rubini, Luyckx, and Meeus (2008).

Internalizing Problem Behavior Symptoms. Anxiety symptoms were measured with the Screen for Child Anxiety Related Emotional Disorders (SCARED). The SCARED is a selfreport questionnaire, which is used to measure symptoms of DSM-IV linked anxiety disorders in children and adolescents. It has been shown to be a reliable and valid measure (Birmaher et al., 1997; Muris, Merckelbach, Van Brakel, \& Mayer, 1999; Muris \& Steerneman, 2001). Its factor structure has consistently been replicated among early and middle adolescents, and boys and girls (Hale, Raaijmakers, Muris, \& Meeus, 2005). The SCARED consists of 38 items and contains five subscales: panic disorder symptoms (13 items), social anxiety symptoms (4 items), separation anxiety symptoms ( 8 items), generalized anxiety symptoms ( 9 items) and school phobia symptoms (4 items). In this study, the composite anxiety score is measured, hence the subscales are not investigated separately. Sample items include 'When frightened, it is hard to breathe', 'I don't like to be with people I don't know', 'I get scared when I sleep away from home', 'I worry about others not liking me' and 'I get headaches or stomach aches when I am at school'. The items are scored on a 3-point scale, ranging from 'hardly ever', 'sometimes' to 'often'

Depressive symptoms were measured with the Children's Depression Inventory (CDI; Kovacs, 1985), a self-report questionnaire aimed at screening (subclinical) depressive 
symptomatology in children and adolescents. This scale has demonstrated convergent and discriminant validity, good internal consistency and adequate test-retest reliability in previous studies (Craighead, Smucker, Craighead, \& Ilardi, 1998; Hodges, 1990). The CDI consists of 27 items (e.g., 'I'm sad all the time'). The items were scored on a 3-point scale, ranging from 1 (false), to 3 (very true).

Externalizing Problem Behavior Symptoms.. Direct aggression was measured with a selfreport aggression questionnaire designed by Björkqvist, Lagerspetz, \& Kaukiainen (1992). Reliability and construct validity have been shown to be strong in adolescent samples (e.g., Hale, Vandervalk, Akse, \& Meeus, 2008). The questionnaire consists of the subscales direct aggression, indirect aggression and withdrawal, but in this study only direct aggression is taken into account. This subscale contains 5 items. Sample items for direct aggression include: When I'm mad at a classmate, I will 'use abusive language about him/her in every situation', 'hit or kick the other' and 'try to trip him/her up'. The items are scored on a 4-point scale, ranging from 'never', 'sometimes', 'often' to 'very often'.

Delinquency was measured with an adapted version of a self-report questionnaire, measuring the frequency of several minor offences (Baerveldt, van Rossem, \& Vermande, 2003). The use of self-report data is widespread in criminology, and it is a valid instrument when restricted to petty crime (Baerveldt, 2000). Adolescents were asked how many times they had committed 14 minor offences, such as 'being caught by the police for doing something bad' or 'stealing a bike', in the past 12 months. The corresponding 14 items were scored on a 4-point scale, ranging from 1 (never), to 4 (four times or more). The original scale by Baerveldt et al. (2003) also contained items measuring drug use. These items were excluded from the delinquency scale.

Drug use was measured with one item from the previously mentioned self-report questionnaire by Baerveldt et al. (2003). Adolescents were asked how many times they had used marihuana and hash in the past 12 months. This item was scored on a 4-point scale, ranging from 1 (never), to 4 (four times or more).

Conflict. The Interpersonal Conflict Questionnaire (Laursen, 1993, 1995) was used to measure the frequency of conflicts. Adolescents indicated whether they had had an argument or fight with their fathers and mothers over the past 7 days with regard to 35 issues. A 5-point Likert scale was used, ranging from never to often. Sample items are 'manners', 'privacy', 'not doing what you are asked to do', 'homework', and 'being honest'. The last item included 
the option "other," which adolescents could use to fill out an additional conflict topic not covered by the list. Similar to previous studies (e.g., van Doorn, Branje, \& Meeus, 2008), we averaged the 35 items to compute mean scores for conflict with fathers and mothers.

\subsubsection{RADAR Sample and Design}

RADAR (e.g., Eichelsheim et al., 2009) is an ongoing longitudinal study with a main focus on development of relationships, problem behaviour, personality and identity. The sample comprises 651 adolescents that participate in annual measurements. Of these 651 adolescents, 626 (54.8\% boys; mean age 13.1 years at the initial measurement occasion) are engaged in three additional internet measurement weeks per year. The remaining 25 participants, who had been selected for the study because they had been victims of violent acts, did not participate in internet assessments to limit the burden on these vulnerable adolescents. Each internet measurement week comprises five consecutive daily measurement occasions of identity, self-concept, relationship quality, and mood.

\subsubsection{RADAR Measures}

Commitment-Reconsideration Dynamic: Identity at the Macro Level. To measure

Commitment and Reconsideration we used the same measure of identity formation as we did in the CONAMORE study (U-MICS; see paragraph 1.5.2). However, instead of focusing on three identity dimensions, we now focused on the two most salient dimensions for early adolescents (i.e., commitment and reconsideration). We again studied one ideological and one interpersonal domain, thus a total of 16 items were used to assess commitment (10 items) and reconsideration (6 items).

Commitment-Reconsideration Dynamic: Identity at the Micro Level. To measure identity on a daily basis (i.e., at the micro level), we used a single-item version of U-MICS developed by Meeus (Crocetti, Rubini, \& Meeus, 2008). Similar to the former version of U-MICS, 5-point Likert-scale items with a response format ranging from 1 (completely untrue) to 5 (completely true) were used. Ideological (i.e., school) and interpersonal (i.e., relation with best friend) domains were assessed with one item for each dimension (i.e., commitment and reconsideration). The items were: "Today, I felt confident about myself because of my education/best friend" (ideological/interpersonal commitment) and "Today, I felt that I could better look for a different school/best friend" (ideological/interpersonal reconsideration). 


\subsubsection{L-TIDES Sample and Design}

L-TIDES (Luyckx, Goossens, \& Soenens, 2006) is a 7-wave longitudinal study on psychosocial development in college students from the faculty of Psychology and Educational Sciences at a large Belgian university. The first wave was collected at the end of 2002 and the interval between each of the waves was six months. The main focus of the study is on the development of identity, personality, and psychosocial adjustment. At the initial measurement occasion, a total of 565 college students $\left(85.3 \%\right.$ female, $14.7 \%$ male; $M_{\text {age }}=18.63$ years $(S D$ $=.61)$ participated.

\subsubsection{L-TIDES Measures}

Personality. As a measure of Costa and McCrae's (1992) Five Factor Model of personality, participants completed the Dutch version of the well-established 60-item NEO Five-Factor Inventory (NEO-FFI; Hoekstra, Ormel, \& De Fruyt, 1996). The five personality factors are measured with 12 items each, such as 'Sometimes I feel completely useless' (Neuroticism), 'I enjoy being around other people' (Extraversion), 'I often try new and foreign food' (Openness), 'In general, I try to be attentive and caring' (Agreeableness), and ' I keep my belongings clean and tidy' (Conscientiousness).

Problem Behavior. We used a 12-item shortened version of the Center for Epidemiologic Studies Depression Scale (CES-D; Radloff, 1977) to assess depressive symptoms. Items were scored on a 4-point Likert-type rating scale (ranging from 'seldom' to 'most of the time or always') and refer to cognitive, somatic and psychological symptoms of depression.

Participants were asked to indicate how often they experienced these symptoms during the past week. A sample item is 'During the last week, I felt depressed'.

General self-esteem was measured with the Rosenberg Self-Esteem Scale (RSES; Rosenberg, 1965). A sample item is 'I feel that I have a number of good qualities'. This scale contains 10 items scored on a 4-point Likert-type rating scale (ranging from 'does not apply to me at all' to 'applies to me very well').

Delinquency was assessed with 11 items adapted from the Deviant Behavior Scale (DBS; Weinmann, 1992). Participants were asked to indicate on a 5-point Likert-type rating scale (ranging from 'does not apply to me at all' to 'applies to me very well') the degree to which they engaged in deviant behaviors (such as shoplifting and destroying other people's property) during the past 6 months. 


\subsubsection{Delinquent and Clinically Referred Adolescents: Sample}

The delinquent and clinically referred youth assessed in chapter 9 were contacted in the institutions in which they resided. The juvenile delinquent sample comprised of 30 boys (16.83 years of age, $S D=2.03$ ) residing in a penitentiary youth institution in the Netherlands. All were referred to the institution by a Dutch court. Our sample of clinically referred youth included 29 adolescents $(15.45$ years of age, $S D=1.06)$ residing in a residential youth institution in the Netherlands. These youth required institutional care because their parents were no longer able to provide adequate care, because of severe behavioural problems of the adolescent themselves or because of their fathers' and/or mothers' lack of adequate parenting skills. They participated in a psychological foster care program, aimed at teaching them the psychological and social skills they would eventually need to earn an independent living. They were compared to 30 adolescents (16.63 years of age , $S D=1.87)$ from the general population, matched to the delinquent sample with regard to background characteristics (e.g., age, gender, educational background of the parents). These 30 adolescents were derived from the previously mentioned CONAMORE project.

\subsubsection{Delinquent and Clinically Referred Adolescents: Measures}

We used the same identity questionnaire (U-MICS) as we did in the CONAMORE study. For a description of this measure, the reader is referred to paragraph 1.5.2.

\subsection{Outline of the dissertation}

In the next eight chapters, the research questions introduced in paragraphs 1.3 and 1.4 are addressed in eight empirical studies. The final chapter of this dissertation contains a summary of the results obtained in the eight empirical studies, and a general discussion of these studies. Finally, a reference list, summaries (in English and in Dutch), an acknowledgment, a curriculum vitae (in English and in Dutch), and my publication list are presented. 


\section{Chapter 2}

Maturation of Personality in Adolescence ${ }^{2}$

${ }^{2}$ Klimstra, T. A., Hale III, W. W., Raaijmakers, Q. A. W., Branje, S. J. T., \& Meeus, W. H. J. (2009). Maturation of personality in adolescence. Journal of Personality and Social Psychology, 96, 898-912. 


\begin{abstract}
The present research assesses adolescent personality maturation by examining three measures of change and stability (i.e., mean-level change, rank-order stability, and profile similarity) of Big Five personality traits, employing data from a five-annual-wave study with overlapping early to middle ( $n=923)$ and middle to late $(n=390)$ adolescent cohorts. Results indicated that mean levels of Agreeableness and Emotional Stability increased during adolescence. There was mixed evidence for increases in Extraversion and Openness. Additionally, rank-order stability and profile similarity of adolescent personality traits clearly increased from early to late adolescence. For all change facets, we found evidence for gender differences in the timing of adolescent personality maturation, as girls were found to mature earlier than boys.
\end{abstract}




\subsection{Introduction}

Adolescence is generally regarded as being the formative period in a person's life. In this period, individuals physically transform from a child to an adult (Petersen, Crockett, Richards, \& Boxer, 1988). These physical changes are paralleled by psychosocial changes: a transition takes place from being a child relying strongly on his or her parents' teachings to becoming an adult who makes his or her own informed decisions (Erikson, 1950). Adolescence has been shown to be a period where individuals, for example, gain an increasingly more stable identity status (Meeus, Iedema, Helsen, \& Vollebergh, 1999), form stable cultural orientations (Vollebergh, Iedema, \& Raaijmakers, 2001), and establish increasingly more salient and intimate relations with peers and romantic partners (Furman \& Buhrmester, 1992). It is likely that these changes towards maturation in biological and psychosocial domains are also reflected by changes in personality traits.

Adolescent personality maturation should be indicated by changes, signified by (normative) growth of personality traits (e.g., Caspi, Roberts, \& Shiner, 2005). Maturation should also be reflected by increases in stability, as inter-individual differences should become more settled (e.g., Costa \& McCrae, 1994), and personality profiles should become more stable and better organized (e.g., Roberts, Caspi, \& Moffitt, 2001). Nevertheless, adolescent personality maturation, as measured by indices of change and stability, has received much less attention than adult personality maturation (Caspi et al., 2005). In the last decade, this situation has changed as an ever-increasing number of studies have focused on maturation of personality during adolescence. Despite this increased attention, studies that longitudinally track personality maturation across adolescence on an annual basis in large population samples are still lacking. Therefore, the current study will set out to assess various facets of change and stability in personality maturation across the entire period of adolescence (i.e., ages 12 to 20), using five-annual wave data on overlapping early to middle adolescent and middle to late adolescent cohorts.

\section{Measuring Personality Maturation in Adolescence}

Throughout the last two decades there has been a growing consensus on the higher-order structure of personality, as a majority of researchers now agree that personality can be subsumed in five broad traits: the Big Five (Caspi et al., 2005). Big Five personality traits include Extraversion, Agreeableness, Conscientiousness, Emotional Stability, and Openness to Experience (McCrae \& Costa, 1987). Extraversion refers to dominance and activity in 


\section{Maturation of Personality}

interpersonal situations, Agreeableness refers to the willingness to maintain positive and reciprocal relationships with others, Conscientiousness refers to organizational and motivational aspects of a person's behaviour, Emotional Stability indicates the ability to deal effectively with negative emotions, and Openness to Experience refers to how a person deals with new information at a personal and experiential level. It has been demonstrated that the Big Five traits apply to adolescent personality in the same way these traits do to adult personality (Digman \& Inouye, 1986; John, Caspi, Robins, Moffitt, \& Stouthamer-Loeber, 1994), and that the use of self-reports is a reliable and valid method to measure Big Five traits in adolescents (e.g., De Fruyt, Mervielde, Hoekstra, \& Rolland, 2000; Soto, John, Gosling, \& Potter, 2008).

Several recent empirical studies (Akse, Hale, Engels, Raaijmakers, \& Meeus, 2007; Branje, Van Lieshout, \& Gerris, 2007; De Fruyt et al., 2006; McCrae et al., 2002; Pullmann, Raudsepp, \& Allik, 2006; Roberts et al., 2001) and meta-analyses (Roberts \& DelVecchio, 2000; Roberts, Walton, \& Viechtbauer, 2006) have examined aspects of adolescent personality maturation. For this purpose, these studies have focused on the three earlier mentioned indicators of maturation: (1) mean-level growth (i.e., increases) on the Big Five traits, (2) an increase of rank-order stability of Big Five traits, and (3) a more stable and consistent organization of the personality profile (i.e., increases in profile similarity). These three indicators of maturation will now be explored.

\section{Mean-Level Change}

A large number of longitudinal studies on mean-level change is summarized in a metaanalysis by Roberts, Walton, and Viechtbauer (2006). They demonstrated that adolescents generally become more Extraverted, more Emotionally Stable, and more Open to Experience as they grow older. The adolescent personality traits of Agreeableness and Conscientiousness tend to stay relatively stable over time. There is, however, a lack of agreement on the amount and the direction of change, as the statistic indicating heterogeneity of results among the studies included in the meta-analysis reached significance for all Big Five dimensions, except for Emotional Stability. There are several possible causes for this heterogeneity: (a) several studies included a limited number of participants $(n<100)$, limiting the generalizability of results from specific studies; (b) a vast majority of the included longitudinal studies had a high attrition rate $(>20 \%)$, (c) studies were published across a long period of time (19682004), possibly causing birth cohort effects (e.g., Twenge, 2000, 2001); and, as the authors 
themselves already indicated: (d) a wide variety of measures was used that were not all specifically designed to measure the Big Five, thereby causing measurement variance.

Recent longitudinal studies on adolescent personality development (Branje et al., 2007; De Fruyt et al., 2006; McCrae et al., 2002; Pullmann et al., 2006) that were not included in the meta-analysis of Roberts (2006) all use measures exclusively designed to measure the Big Five. Nevertheless, these studies also show considerable heterogeneity of findings. One study, specifically focusing on early adolescents (De Fruyt et al., 2006), found increases in Emotional Stability, decreases in Openness and Conscientiousness, and stable levels of Extraversion and Agreeableness. Two other studies focused on the period from early to middle adolescence (Branje et al., 2007; McCrae et al., 2002). Both studies found increases in Openness and stable levels of Emotional Stability, but did not agree on the developmental course of the other three dimensions. Whereas Branje et al. (2007) found decreases in Extraversion, but increases in Agreeableness and Conscientiousness, McCrae et al. (2002) found stable levels of Extraversion and Agreeableness, and decreases in Conscientiousness. These inconsistencies might be due to specific sample characteristics, as the sample of McCrae et al. (2002) only included gifted students, and the sample of Branje et al. (2007) only included adolescents from middle-class two parent families with at least two children. Finally, one study (Pullmann et al., 2006) compared personality changes in early, middle, and late adolescents. Overall, they found more mean-level change in early and middle adolescence than in late adolescence. Specifically, their early adolescents increased in Emotional Stability and Extraversion, decreased in Agreeableness, and had stable levels of Openness and Conscientiousness. The middle adolescents displayed increases in Emotional Stability, Extraversion and Openness, but stable levels of Agreeableness and Conscientiousness. Late adolescents increased in Openness, but exhibited no changes on the other four Big Five traits. Although the study by Pullmann et al. (2006) provides valuable information on adolescent personality maturation by covering the entire adolescent period, they were only able to measure personality traits twice in the same individuals. Therefore, they were unable to test whether personality maturation is described by a linear or a curvilinear pattern. In addition, their attrition rates were high (22\%-57\%), which could have had an influence on their results.

In sum, the meta-analysis (Roberts et al., 2006) and the recent empirical studies (Branje et al., 2007; De Fruyt et al., 2006; McCrae et al., 2002; Pullmann et al., 2006) agree that levels of Openness increase after early adolescence, while there is also strong evidence for increasing levels of Emotional Stability. Concerning the other three dimensions, there is less agreement on mean-level change. Nevertheless, when considering the results of the 


\section{Maturation of Personality}

mentioned studies the number of Big Five dimensions where mean-level increases, and thus maturation was found, exceeds the number of dimensions where decreases were found. Overall, previous studies suggest that when personality traits change, they tend to change in the direction of maturation, although there are inconsistencies regarding the specific traits that mature and regarding the period of adolescence in which maturation takes place.

\section{Rank-order Stability}

Indices of rank-order stability indicate whether the rank-order of individuals on a certain trait is maintained over time. Mean-levels on a certain trait might change, but the rank-order can remain stable at the same time. Hence, mean-level change and rank-order stability can be interpreted as two independent constructs (Roberts \& DelVecchio, 2000; Roberts et al., 2006). If mean-level changes are accompanied by high rank-order stability, the observed mean-level changes reflect normative change. Normative changes are changes that occur to a similar degree in most people in the population, and they are considered to reflect universal maturation processes (e.g., Helson, Kwan, John, \& Jones, 2002; Kasen, Chen, Sneed, Crawford, \& Cohen, 2006; Roberts et al., 2006). Therefore, to asses whether there are such universal maturation processes, indices of rank-order stability need to be assessed alongside indices of mean-level change. In addition, an increase in rank-order stability can, by itself, also be interpreted as maturation, since inter-individual differences should become more set with age (Costa \& McCrae, 1994).

A meta-analysis by Roberts and DelVecchio (2000), and empirical studies by Akse et al. (2007) and Pullmann et al. (2006) indicated that rank-order stability of personality indeed increases as adolescents grow older. However, the meta-analysis by Roberts and DelVecchio (2000) not only used Big Five measures, but considered a wide range of measures such as ego-identity questionnaires, temperament scales, and Rorschach tests. Akse et al. (2007) and Pullmann et al. (2006) only had a limited number of measurement occasions for rank-order stability (i.e., two and three, respectively), and could therefore not systematically examine increases. In sum, the studies that are available up to now indicate that rank-order stability of personality traits increases as adolescents grow older, just as Costa and McCrae (1994) predicted. However, the aforementioned studies only have a limited number of measurement occasions and do not provide information that helps us to understand exactly when in adolescence personality traits start to become more settled. For that purpose, longitudinal studies systematically assessing year-to-year changes in rank-order stability in adolescent Big Five traits are needed. 


\section{Profile Similarity}

Profile similarity provides information on the stability of a constellation of traits over time within a person, and is therefore, unlike rank-order stability and mean-level change, not informative on changes in individual personality traits. It is also different from other change indices, because profile similarity indicates to what extend a person's personality configuration changes over time, and is not indicative of changes at the sample level. Profile similarity is usually calculated with $q$-correlations. Like Pearson correlations, $q$-correlations range from -1 to 1 . The higher the $q$-correlation, the more stable a constellation of traits within a person is (e.g., Ozer \& Gjerde, 1989; Roberts et al., 2001). A more stable constellation of traits indicates that a person's personality profile is well organized, and is therefore an indicator of maturation (Roberts et al., 2001).

Previous studies on profile similarity of personality by Block (1971), Roberts et al. (2001), and De Fruyt et al. (2006; the only Big Five study on adolescent profile similarity) found average $q$-correlations exceeding .70 in adolescence. The only study reporting on longitudinal changes in profile similarity from early adolescence to late adolescence (Ozer \& Gjerde, 1989), found that profile similarity was stable across time. However, since only a small sample of adolescents $(n=84)$ was employed, the generalizability of these results is debatable. Large-scale longitudinal studies reporting on systematic increases of profile similarity in adolescents are not yet available.

\section{The state of the art of studies on personality maturation in adolescence}

Our discussion of research on the three change measures reveals that there have been quite a few studies examining aspects of personality maturation in adolescence, specifically during the last decade. However, studies on mean-level change do not agree on exactly what Big Five dimensions maturation processes are most evident, there are no studies available that can inform us on exactly where in adolescence inter-individual differences in personality traits start to become more settled, and large-scale longitudinal studies systematically tracking changes in profile similarity are not yet available. In addition, most of the mentioned studies did not asses all three indicators of maturation (i.e., increases on the Big Five traits, increasingly stable rank-order with regard to inter-individual differences, and a better organized personality profile) in one design, although it is only possible to gain a comprehensive perspective on maturation of personality if all three indicators are assessed (e.g., Roberts et al., 2001; Caspi \& Roberts, 1999). The one study (De Fruyt et al., 2006) that did examine all three indicators, only assessed maturation in childhood and early adolescence. 


\section{Maturation of Personality}

The three indicators of maturation have so far not been assessed together in studies covering other periods in adolescence.

Another issue is that a majority of the published studies, including the one by De Fruyt et al. (2006), only measured adolescent personality traits twice for the same participants. Changes found in studies with only two longitudinal measurement occasions may be caused by temporary factors that influence scores on one of the measurement occasions, whereas consistent change across several measurement occasions is more likely to reflect reliable developmental trends. Consequently, the reliability of change trajectories has been shown to increase steadily as the number of measurement occasions increases (Willett, Singer, \& Martin, 1998). In addition, two measurement occasions only allow for assessment of linear change, which implies that it is possible that no changes are found if the underlying pattern is curvilinear. Finally, studies with only two measurement occasions are unable to investigate systematic increases in rank-order stability and profile similarity.

In sum, to advance the knowledge on adolescent personality maturation, studies that longitudinally and systematically measure various indicators of personality change and stability in a large and broad sample of adolescents, using multiple measurement waves, are needed. The current study aims to provide such a comprehensive perspective on personality maturation.

\section{Gender Differences in Personality}

Since previous studies found gender differences in adolescent personality change, we will also consider the role of gender in adolescent personality maturation. We will now discuss the gender differences found by these previous studies.

With regard to the first aspect of personality maturation (i.e., increases in mean-levels of Big Five traits), three of the aforementioned longitudinal studies on mean-level change in adolescent personality traits explicitly examine gender differences. Two of those studies compared overall mean-levels of personality traits of adolescent boys and girls. Branje et al. (2007) found that boys tended to be more extraverted and open to experience than girls, whereas McCrae et al. (2002) concluded the exact opposite. In addition, McCrae et al. (2002) found higher levels of Agreeableness and lower levels of Emotional Stability in girls when compared to boys, whereas Branje et al. (2007) found no gender differences on these two dimensions. With regard to changes, there is also little agreement among studies. Branje et al. (2007) found decreases for boys, and increases for girls in Extraversion. For Agreeableness, Conscientiousness, and Openness, they found stability for boys, but increases for girls. Both 
studies agreed that there were no gender differences in Conscientiousness. The other two longitudinal studies reporting on gender differences (McCrae et al., 2002; Pullmann et al., 2006), found a more advantageous developmental pattern for Emotional Stability for boys than for girls, as Pullmann et al. (2006) reported increases for boys and stability for girls, and McCrae et al. (2002) reported stability for boys and decreases for girls. Emotional Stability was the only dimension where Branje et al. (2007) did not find gender differences in change rates. Thus, there is little agreement on gender differences in both mean-levels and mean-level changes in Big Five traits.

Gender differences concerning the second aspect of maturation (i.e., increases in rankorder stability) were examined in three studies. In their meta-analysis, Roberts and DelVecchio (2000) did not find gender differences in rank-order stability across the life span. Due to the underrepresentation of studies assessing gender differences in personality change in the meta-analysis, they were unable to focus on a specific period, such as adolescence. Furthermore, studies that specifically focused on adolescents either found that girls were somewhat more stable than boys (Pullmann et al., 2006), or found no gender differences (McCrae et al., 2002). However, because none of the aforementioned studies systematically measured year-to-year changes in rank-order stability in adolescence, it is not possible to use these studies to explore whether inter-individual differences in personality traits start to become more set at a similar age for adolescent boys and girls. To examine such possible gender differences in the timing of longitudinal changes in rank-order stability for boys and girls, studies that assess year-to-year changes in rank-order stability are needed.

Finally, with respect to the third aspect of adolescent personality maturation (i.e., an increasingly more organized personality profile), only two studies have examined gender differences. Ozer and Gjerde (1989) found no substantial differences, while Roberts et al. (2001) reported higher profile similarity in girls, when compared to boys. However, as previously mentioned, the former study employed a small sample and the latter focused on the transition from adolescence into adulthood. In addition, neither of these two studies focused on Big Five trait profiles. Thus, gender differences in mean-levels and increases in Big Five profile similarity still need to be investigated.

In sum, previous studies have examined gender differences for all three aspects of adolescent personality maturation. However, there is little agreement (i.e., for mean-level change), or mixed evidence (i.e., for rank-order stability and profile similarity) with regard to gender differences in personality change. These inconsistencies in findings of previous studies could be due to the possibility that gender differences mainly exist in the timing of personality 


\section{Maturation of Personality}

development, as suggested by Branje et al. (2007). They proposed that the gender differences they found at age 16 (i.e., more increases in girls than in boys, and hence higher mean-levels on several personality traits for girls) could be caused by a pubertal timing effect in personality. Branje et al. (2007) therefore suggested that boys could catch up with girls with regard to personality in late adolescence. However, they were unable to test this hypothesis. Such differences in the timing of personality maturation might exist, as there are profound gender differences in indices of pubertal timing and neurological maturation processes.

On one of the most used indicators of pubertal timing, peak height velocity (i.e., the age at which an adolescent exhibits the fastest growth rate), girls have been shown to be two years ahead on average, when compared to boys (e.g., Beunen et al., 2000; Petersen et al., 1988). In addition, brain development in several areas is up to one year ahead in girls (Giedd et al., 1999; Lynn, 1994). Due to these gender differences in pubertal timing and neurological development, girls usually look more mature and have a cognitive advantage (Colom \& Lynn, 2004) in the earlier stages of adolescence. In a review, Paikoff and Brooks-Gunn (1991) indicated that adolescents who mature earlier are also ahead on several factors related to Big Five traits, such as social maturity, peer-prestige, leadership and self-esteem. Gender differences in pubertal timing and neurological maturation are, therefore, likely to be reflected in gender differences in Big Five personality maturation.

\section{Hypotheses}

In sum, we will examine whether adolescent personality maturation is reflected by: (1) increasing mean-levels of Big Five traits, (2) increasingly more settled inter-individual differences, (3) and better organized personality profiles. We expect increasing mean-levels of Big Five traits through adolescence, but also substantial inter-individual differences in these increases. Previous studies consistently found evidence for increases in Openness in adolescence, but it is unclear in exactly which other traits maturation is reflected. Increasingly more settled inter-individual differences are expected to be signified by increases in rankorder stability of personality traits, whereas a better organized personality profile is expected to be indicated by increases in $q$-correlations. Finally, we expect gender differences in the timing of all three aspects of personality maturation. Specifically, girls should exhibit high mean-levels, rank-order stability, and profile similarity of personality at an earlier stage in adolescence than boys. 


\subsection{Method}

\section{Participants}

Data for this study were collected as part of a five-wave longitudinal research project on Conflict And Management Of Relationships (CONAMORE; Meeus et al., 2006), with a oneyear interval between each of the waves. The longitudinal sample was comprised of 1,313 adolescents, who were divided into an early to middle adolescent cohort ( $n=923 ; 70.3 \%)$, who were 12.4 years of age on average $(S D=.59)$, and a middle to late adolescent cohort $(n=$ $390 ; 29.7 \%)$ with an average age of 16.7 years $(S D=.80)$ during the first wave of measurement. Because both age groups were assessed during five measurement waves, a total age range from 12 to 20 years was available.

The early to middle adolescent cohort consisted of 468 boys (50.7\%) and 455 girls (49.3\%), and the middle to late adolescent cohort consisted of 169 boys (43.3\%) and 221 girls (56.7\%). In the younger cohort, $85.1 \%$ indicated that they were living with both their parents, and in the older cohort a similar percentage of adolescents reported the same (84.3\%). Other adolescents lived with their mother $(7.9 \%$ and $7.2 \%$ in the younger and older cohort, respectively) or elsewhere (e.g., with their father, with one biological parent and one stepparent, or with other family members). The composition of the two cohorts did not significantly differ with regard to ethnicity. In the younger cohort, $83.4 \%$ identified themselves as Dutch and 16.6\% indicated that they belonged to ethnic minorities (e.g., Surinamese, Antillean, Moroccan, Turkish). In the older cohort these figures were $87.4 \%$, and $12.6 \%$, respectively. In the year that the current study was initiated (2001), $21 \%$ of the Dutch early to middle adolescents and $22 \%$ of the Dutch middle to late adolescents belonged to ethnic minorities (Statistic Netherlands, 2008a). Thus, ethnic minorities were slightly underrepresented in our sample. With regard to education, all participants initially were in junior high and high schools. Given the Dutch educational system, most participants changed school at least once during the study. Specifically, participants in the younger cohort switched from junior high school to high school, whereas most of the participants in the older cohort switched from high school to college. Because of the sample recruitment procedure, $100 \%$ of our middle to late adolescents were in high school or college, whereas national demographic statistics (Statistic Netherlands, 2008a, 2008b, 2008c) reveal that 96\% of the Dutch middle to late adolescents was in some form of education at that age during the period covered with the current study (i.e., 2001-2005). 
Sample attrition was $1.2 \%$ across waves: in waves $1,2,3,4$, and 5 the number of participants was 1,313, 1,313 (923 early to middle adolescents and 390 middle to late adolescents), 1,293 (923 early to middle adolescents and 370 middle to late adolescents), 1,292 (923 early to middle adolescents and 369 middle to late adolescents) and 1,275 (913 early to middle adolescents and 362 middle to late adolescents), respectively. For each analysis we used the optimal method to estimate missing values. For the Latent Growth Curve Analyses (used to asses mean-level change), we therefore used the Full Information Maximum Likelihood (FIML) procedure in Mplus (Muthén \& Muthén, 2007). As the analyses concerning rank-order stability and profile similarity were run in SPSS, where FIML is not available, we used the EM-procedure to estimate missing values for these analyses. Across waves $4.9 \%$ of the data was missing. Little's Missing Completely At Random Test (Little, 1988), a regular test to compare imputed with non-imputed data, revealed a normed $\chi^{2}$ $\left(\chi^{2} / \mathrm{df}\right)$ of 1.35 . According to guidelines by Bollen (1989), this indicates a good fit between sample scores with and without imputation.

\section{Procedure}

The participating adolescents were recruited from several, randomly selected schools in the province of Utrecht, The Netherlands. Of the 20 schools that were approached, 12 schools $(60 \%)$ decided to participate. There were no general differences between participating and non-participating schools. All offered comparable educational programs. We were unable to collect data on personality or other variables among the pupils of the non-participating schools. Participants and their parents received an invitation letter, describing the research project and goals, and explaining the possibility to decline from participation. More than $99 \%$ of the approached students decided to participate. All participants signed the informed consent form. The questionnaires were completed at the participants' own (junior) high school or at home, during annual assessments. Confidentiality of responses was guaranteed. Verbal and written instructions were offered. The adolescents received $€ 10$ (approximately US \$15) as a reward for every wave they participated in.

\section{Measures}

Personality. Personality was assessed with a shortened 30-item Dutch version of Goldberg's Big Five questionnaire (Gerris et al., 1998; Goldberg, 1992). In this instrument, a 7-point likert scale, with a response format ranging from 1 (completely untrue) to 7 (completely true), is used to assess five personality dimensions: Extraversion, Agreeableness, 
Conscientiousness, Emotional Stability, and Openness to Experience. All dimensions are measured with 6 items each, such as: talkative (Extraversion), sympathetic (Agreeableness), systematic (Conscientiousness), worried (Emotional Stability, reversed scored), and creative (Openness to Experience). Previous studies (e.g., Branje, van Lieshout, \& van Aken, 2004; De Fruyt et al., 2006; Dubas, Gerris, Janssens, \& Vermulst, 2002; Scholte, van Aken, \& van Lieshout, 1997) have demonstrated that this measure provides a valid and reliable estimate of adolescent Big Five Personality Traits. In the current study, reliability was high across waves, as the ranges of internal consistency coefficients (Cronbach's alphas) for each Big Five Trait in early to middle adolescents were: Extraversion (.76 - .85), Agreeableness (.80 - .88), Conscientiousness (.81 - .88), Emotional Stability (.81 - .84), and Openness to Experience (.76 - .78). For middle to late adolescents, these figures were: Extraversion (.86 - .91), Agreeableness (.81 - .88), Conscientiousness (.87 - .92), Emotional Stability (.79 - .85), and Openness to Experience (.74 - .79).

\section{Strategy of Analyses}

We examined three types of change and stability (i.e., mean-level change, rank-order stability, and profile similarity) in early to middle and middle to late adolescents, and examined gender differences in these change measures. Mean-level change was estimated with Latent Growth Curve Modeling (LGCM; e.g., Duncan, Duncan, Stryker, Li, \& Alpert, 1999), using Maximum Likelihood estimation (ML) in Mplus (Muthén \& Muthén, 2007). LGCM provides mean initial levels (i.e., intercepts) and mean change rates (i.e., slopes), which are based on individual growth trajectories of all participants. To indicate the extent to which there are inter-individual differences in growth, intercept and slope variances are also incorporated in the analysis and provided in the output. Therefore, LGCM integrates individual-level change and mean-level change in one analysis (e.g., Branje et al., 2007). Different shapes of development can be tested, as linear and curvilinear shapes of development can be specified with the slopes. Because latent variables are used in LGCM to estimate mean-level change, it controls for measurement error (e.g., Duncan et al., 1999), which is not possible with classical techniques, such as Repeated Measures ANOVA, used in a majority of previous studies on adolescent personality maturation (except for Branje et al. (2007) who also used LGCM).

To assess gender differences in mean-levels and change, we used a multigroup design, with four groups: early to middle adolescent boys and girls, and middle to late adolescent boys and girls. All participants in the younger cohort were in their first year of secondary education at $\mathrm{T} 1$ and in their fifth year of secondary education at T5, whereas the participants 
from the older cohort were in their fifth year of secondary education at T1. Measurement wave was fully confounded with the number of years adolescents had experienced secondary education (i.e., grade level), and was also largely confounded with age (see sample description). No data were available concerning the exact chronological age of the participants. Therefore, we used measurement occasion, which was fully confound with grade level, to specify our growth models. For convenience of presentation, we will refer to the stage of adolescence (i.e., early, middle and late adolescence), instead of measurement wave or grade level, when discussing our results.

One wave of overlap existed between the two age cohorts. During this one wave of overlap (i.e., T5 for early to middle adolescents and T1 for middle to late adolescents), adolescents were 16 years of age on average, and all had experienced 5 years of secondary education. Therefore, we placed the intercept at T5 for early to middle adolescents, and at T1 for middle to late adolescents (i.e., slope factor loadings were $-4,-3,-2,-1$, and 0 for the five consecutive measurement occasions for early to middle adolescents, and 0, 1, 2, 3, and 4 for middle to late adolescents). We used the same growth factor loadings for boys and girls. The kind of growth model that best applied to our data was chosen by testing models with linear growth, as well as models with curvilinear growth, for all Big Five traits separately. We used the unconstrained models to determine growth in the various groups (i.e., early to middle adolescent boys and girls, and middle to late adolescent boys and girls), and used chi-square difference tests to assess differences between these groups in growth estimates (i.e., means and variances of intercepts and slopes). Model fit of the various models was judged by assessing Root Mean Square Errors of Approximation (RMSEA's), Comparative Fit Indices (CFI's), and Tucker-Lewis Indices (TLI's). RMSEA's below .08, and CFI's and TLI's over .95 indicate an adequate model fit; relatively lower RMSEA's, and higher CFI's and TLI's indicate a better fit when comparing models (Kline, 1998). Intercepts and slopes within the same model were allowed to correlate, as this significantly improved the fit of all models.

Pearson correlations were used to assess rank-order stability. To test for gender differences and age-related increases in rank-order stability, correlation coefficients were transformed to $z$-scores using Fisher's $r$-to- $z$ transformation. Gender differences in $z$-scores were tested for significance by comparing a test-retest $z$-score for a certain measurement wave interval within an age cohort for boys, with $z$-scores obtained for girls at that same measurement wave interval within the same age cohort (e.g., the T1-T2 $z$-score for early to middle adolescent boys was compared to the T1-T2 $z$-score for early to middle adolescent girls). To test for age effects, we compared $z$-scores for the same between-wave interval for 
the two cohorts within gender cohorts (e.g., the T1-T2 $z$-scores obtained for early to middle adolescent girls were compared to the T1-T2 $z$-scores for middle to late adolescent girls).

Profile similarity was assessed with $q$-correlations (e.g., Block, 1971; Roberts et al., 2001). A $q$-correlation was calculated for each individual separately, by correlating a rankordered set of Big Five traits at one measurement occasion (e.g., T1) with a rank-ordered set of the same Big Five traits at the subsequent measurement occasion (e.g., T2). Biesanz, West, and Kwok (2003) proposed that LGCM can be used to assess mean-level changes in profile similarity, if enough measurement occasions are available. As this was the case in the current study, we conducted LGCM to assess age-related increases in mean $q$-correlations. Since profile similarity was measured from $\mathrm{T} 1$ to $\mathrm{T} 2, \mathrm{~T} 2$ to $\mathrm{T} 3, \mathrm{~T} 3$ to $\mathrm{T} 4$, and $\mathrm{T} 4$ to $\mathrm{T} 5$, four measurement occasions were available for each cohort. We chose a multigroup design with four groups: early to middle adolescent boys and girls, and middle to late adolescent boys and girls (for the four consecutive measurement occasions, factor loadings were $0,1,2$, and 3 , for all groups). To assess gender and age effects, and to judge model fit, we followed the same procedure previously described for the LGCMs on mean-level change.

\subsection{Results}

\section{Mean-level Change}

To assess mean-level change, we ran a set of five univariate multigroup Latent Growth Curve Models to assess personality change and gender differences herein. Four groups were distinguished: early to middle adolescent boys and girls, and middle to late adolescent boys and girls. Observed means and standard deviations of these groups are provided in Table 2.1. For all Big Five factors, the best fitting and most parsimonious models were models for curvilinear growth. In curvilinear models, growth is a combination of linear and curvilinear slopes. Because that makes it hard to judge growth patterns from a table only, estimated growth of Extraversion, Agreeableness, Conscientiousness, Emotional Stability, and Openness is displayed in Figure 2.1a (boys) and Figure 2.1b (girls). Fit indices of the unconstrained models appear in Table 2.2. Growth parameters of the unconstrained models, and between-group differences as tested with Chi-square difference tests, are displayed in Table 2.3.

We found only some evidence for increases in Extraversion in early to middle adolescence. There was, however, a cohort effect, as levels of Extraversion were lower in the middle to late adolescent cohort than in the early to middle adolescent cohort. There was little 
Maturation of Personality

Table 2.1

Observed Means and Standard Deviations of Personality in Early to Middle Adolescent boys $(N=468)$ and girls $(N=455)$ and Middle to Late Adolescent boys $(N=169)$ and girls $(N=221)$.

\begin{tabular}{|c|c|c|c|c|c|c|c|c|c|c|}
\hline & \multicolumn{10}{|c|}{ Early to Middle Adolescence } \\
\hline & \multicolumn{2}{|c|}{ T1 } & \multicolumn{2}{|c|}{$\mathrm{T} 2$} & \multicolumn{2}{|c|}{ T3 } & \multicolumn{2}{|c|}{ T4 } & \multicolumn{2}{|c|}{ T5 } \\
\hline & $M$ & $S D$ & $M$ & $S D$ & $M$ & $S D$ & $M$ & $S D$ & $M$ & $S D$ \\
\hline \multicolumn{11}{|l|}{ Boys } \\
\hline E & 4.87 & .98 & 4.75 & .97 & 4.89 & .98 & 4.88 & 1.06 & 4.96 & 1.08 \\
\hline A & 4.93 & 1.16 & 5.05 & 1.03 & 5.00 & .94 & 5.14 & .96 & 5.31 & .78 \\
\hline $\mathrm{C}$ & 4.05 & 1.13 & 4.16 & 1.18 & 3.93 & 1.13 & 3.89 & 1.16 & 4.01 & 1.19 \\
\hline ES & 4.70 & 1.16 & 4.60 & 1.10 & 4.78 & .97 & 4.81 & .99 & 4.89 & 1.00 \\
\hline $\mathrm{O}$ & 4.38 & 1.14 & 4.56 & 1.10 & 4.40 & 1.00 & 4.45 & 1.04 & 4.57 & 1.01 \\
\hline \multicolumn{11}{|l|}{ Girls } \\
\hline $\mathrm{E}$ & 4.95 & 1.05 & 4.93 & 1.14 & 4.91 & 1.17 & 4.83 & 1.18 & 4.91 & 1.14 \\
\hline A & 5.20 & .97 & 5.37 & .91 & 5.33 & .89 & 5.47 & .74 & 5.57 & .67 \\
\hline $\mathrm{C}$ & 4.23 & 1.09 & 4.29 & 1.14 & 4.25 & 1.16 & 4.28 & 1.17 & 4.35 & 1.19 \\
\hline ES & 4.57 & 1.08 & 4.36 & 1.12 & 4.40 & 1.14 & 4.39 & 1.08 & 4.36 & 1.08 \\
\hline $\mathrm{O}$ & 4.40 & 1.01 & 4.60 & 1.04 & 4.57 & 1.07 & 4.75 & .96 & 4.83 & .94 \\
\hline
\end{tabular}

\begin{tabular}{|c|c|c|c|c|c|c|c|c|c|c|}
\hline & \multicolumn{10}{|c|}{ Middle to Late Adolescence } \\
\hline & \multicolumn{2}{|c|}{ T1 } & \multicolumn{2}{|c|}{$\mathrm{T} 2$} & \multicolumn{2}{|c|}{ T3 } & \multicolumn{2}{|c|}{ T4 } & \multicolumn{2}{|c|}{ T5 } \\
\hline & $M$ & $S D$ & $M$ & $S D$ & $M$ & $S D$ & $M$ & $S D$ & $M$ & $S D$ \\
\hline \multicolumn{11}{|l|}{ Boys } \\
\hline E & 4.63 & 1.20 & 4.66 & 1.08 & 4.78 & 1.01 & 4.87 & 1.08 & 4.93 & 1.13 \\
\hline $\mathrm{A}$ & 5.17 & .98 & 5.49 & .71 & 5.58 & .58 & 5.58 & .59 & 5.68 & .60 \\
\hline $\mathrm{C}$ & 4.10 & 1.20 & 4.18 & 1.21 & 4.22 & 1.10 & 4.23 & 1.12 & 4.32 & 1.18 \\
\hline ES & 4.58 & 1.11 & 4.56 & 1.02 & 4.68 & 1.02 & 4.85 & .94 & 4.77 & .99 \\
\hline $\mathrm{O}$ & 4.72 & 1.08 & 4.90 & .89 & 4.89 & .82 & 4.87 & .93 & 4.95 & .85 \\
\hline \multicolumn{11}{|l|}{ Girls } \\
\hline $\mathrm{E}$ & 4.62 & 1.21 & 4.78 & 1.18 & 4.82 & 1.20 & 4.79 & 1.14 & 4.77 & 1.18 \\
\hline A & 5.50 & .74 & 5.67 & .57 & 5.66 & .61 & 5.73 & .56 & 5.73 & .55 \\
\hline $\mathrm{C}$ & 4.38 & 1.21 & 4.47 & 1.22 & 4.54 & 1.22 & 4.64 & 1.24 & 4.71 & 1.19 \\
\hline ES & 4.09 & .98 & 4.04 & 1.00 & 4.21 & .97 & 4.22 & 1.05 & 4.24 & 1.04 \\
\hline $\mathrm{O}$ & 4.83 & .88 & 4.97 & .84 & 4.91 & .87 & 4.97 & .82 & 4.87 & .86 \\
\hline
\end{tabular}

Note. $\mathrm{E}=$ Extraversion; $\mathrm{A}=$ Agreeableness; $\mathrm{C}=$ Conscientiousness; $\mathrm{ES}=$ Emotional Stability; $\mathrm{O}=$ Openness 
Table 2.2

Fit Indices for Unconstrained Univariate Multigroup Latent Growth Curve Models

\begin{tabular}{lcccccc}
\hline \multicolumn{1}{c}{$\chi^{2}$} & $d f$ & CFI & TLI & RMSEA & $\begin{array}{c}\text { 90\% C.I. of } \\
\text { RMSEA }\end{array}$ \\
\hline Extraversion & $44.80^{* *}$ & 24 & .99 & .99 & .05 & $.03-.07$ \\
Agreeableness & $55.47 * *$ & 32 & .99 & .98 & .05 & $.03-.07$ \\
Conscientiousness & $37.35^{*}$ & 24 & 1.00 & .99 & .04 & $.01-.07$ \\
Emotional Stability & $65.25 * * *$ & 27 & .98 & .98 & .07 & $.05-.09$ \\
Openness & $63.25 * * *$ & 27 & .99 & .98 & .06 & $.04-.09$ \\
\hline Note. ${ }^{*} p<.05, * * p<.01, * * * p<.001$. & & & & &
\end{tabular}

evidence for gender differences in Extraversion. In early to middle adolescence, boys displayed a small curvilinear increase, whereas girls displayed a non-significant increase in Extraversion. These gender differences failed to reach significance. In middle to late adolescence, we found no significant changes for boys, but girls displayed a curvilinear pattern that resulted in very little changes. Therefore, boys and girls had similar levels of Extraversion throughout adolescence.

Adolescents in our sample became much more agreeable as they grew older, evidenced by increases in early to middle and middle to late adolescent boys and girls. These changes usually followed a curvilinear pattern. Only in early to middle adolescent girls, change was described by a linear pattern. In early adolescence, girls had higher levels of Agreeableness than boys. Since both boys and girls displayed similar increases towards middle adolescence, these gender differences were still present in middle adolescence. However, boys exhibited somewhat stronger increases than girls in middle to late adolescence. As a result, gender differences in Agreeableness had almost disappeared by late adolescence.

Levels of Conscientiousness were mostly stable throughout adolescence. There were, however, profound gender differences. Boys began with lower levels of Conscientiousness than girls in early adolescence. Their levels of Conscientiousness decreased in a curvilinear fashion towards middle adolescence, whereas girls displayed stable levels Conscientiousness. In middle to late adolescence the initial gender differences remained, as levels of Conscientiousness did not change significantly for both boys and girls. By late adolescence, girls were still more conscientious than boys. 
Maturation of Personality

Table 2.3

Growth Factors of the Unconstrained Univariate Multigroup Latent Growth Curve Models for Early to Middle and Middle to Late Adolescent Boys and Girls

\begin{tabular}{|c|c|c|c|c|}
\hline \multirow[t]{2}{*}{ Growth Factors } & \multicolumn{2}{|c|}{ Early to Middle Adolescence } & \multicolumn{2}{|c|}{ Middle to Late Adolescence } \\
\hline & Mean & $\sigma^{2}$ & Mean & $\sigma^{2}$ \\
\hline \multicolumn{5}{|l|}{ Boys } \\
\hline \multicolumn{5}{|l|}{ Intercepts } \\
\hline Ex & $4.97 * * *$ a & $.76^{* * * 1}$ & $4.63 * * * b$ & $.89 * * * 1$ \\
\hline $\mathrm{Ag}$ & $5.28 * * * a$ & $.30 * * * 1,2$ & $5.24 * * * a$ & $.26 * * * 2$ \\
\hline $\mathrm{Co}$ & $3.96 * * *$ a & $.95 * * * 1$ & $4.12 * * * a$ & $.86 * * * 1$ \\
\hline ES & $4.92 * * * a$ & $.67 * * * 1,2$ & $4.51 * * * \mathrm{~b}$ & $.64 * * * 1$ \\
\hline $\mathrm{Op}$ & $4.53 * * *$ a & $.77 * * * 1$ & $4.79 * * * b$ & $49 * * * 2$ \\
\hline \multicolumn{5}{|l|}{ Linear Slopes } \\
\hline Ex & $.09 * \mathrm{a}$ & $.07^{1}$ & $.06^{\mathrm{a}}$ & $.25^{* 1,2}$ \\
\hline $\mathrm{Ag}$ & $.18 * * * a, b$ & $.07^{1,2}$ & $.23 * * * \mathrm{a}$ & $.00^{1}$ \\
\hline Co & $.05^{\mathrm{a}}$ & $.10^{1}$ & $.05^{\mathrm{a}}$ & $.12^{1}$ \\
\hline ES & $.11 *^{\mathrm{a}}$ & $.26^{* * 1}$ & $.13 *^{\mathrm{a}}$ & $.00^{2}$ \\
\hline $\mathrm{Op}$ & $.09 * \mathrm{a}$ & $.17 * 1$ & $.05^{\mathrm{a}}$ & $.01^{2}$ \\
\hline \multicolumn{5}{|l|}{ Quadratic Slopes } \\
\hline Ex & $.01^{\mathrm{a}}$ & $.01^{1}$ & $.01^{\mathrm{a}}$ & $.01 * 1$ \\
\hline $\mathrm{Ag}$ & $.24 * \mathrm{a}$ & $.01^{1}$ & $-.03 * * b$ & $.00^{2}$ \\
\hline $\mathrm{Co}$ & $.02 * \mathrm{a}$ & $.01^{1}$ & $.00^{\mathrm{b}}$ & $.00^{1}$ \\
\hline ES & $.01^{\mathrm{a}}$ & $.02 * * * 1$ & $-.01^{\mathrm{a}}$ & $.01^{2}$ \\
\hline Op & $.02^{\mathrm{a}}$ & $.01^{1}$ & $.00^{\mathrm{a}, \mathrm{b}}$ & $.00^{1}$ \\
\hline \multicolumn{5}{|l|}{ Girls } \\
\hline \multicolumn{5}{|l|}{ Intercepts } \\
\hline Ex & $4.91 * * * a$ & $1.29 * * * 2$ & $4.64 * * * b$ & $1.10 * * * 2$ \\
\hline $\mathrm{Ag}$ & $5.56 * * * b$ & $.35 * * * 1$ & $5.54 * * * \mathrm{~b}$ & $.23 * * * 2$ \\
\hline Co & $4.33 * * * \mathrm{~b}$ & $1.15 * * * 1$ & $4.39 * * * \mathrm{~b}$ & $1.14 * * * 1$ \\
\hline ES & $4.37 * * * \mathrm{~b}$ & $.95 * * * 2$ & $4.06 * * * \mathrm{c}$ & $.62 * * * 1$ \\
\hline $\mathrm{Op}$ & $4.81 * * * b$ & $.82 * * * 1$ & $4.85 * * * \mathrm{~b}$ & $.49 * * * 2$ \\
\hline \multicolumn{5}{|l|}{ Linear Slopes } \\
\hline Ex & $.03^{\mathrm{a}}$ & $.29 * * * 2$ & $.14 * * \mathrm{a}$ & $.25 * * 1,2$ \\
\hline $\mathrm{Ag}$ & $.09 * * \mathrm{~b}$ & $.22 * * * 2$ & $.13 * * * a, b$ & $.00^{1}$ \\
\hline $\mathrm{Co}$ & $.06^{\mathrm{a}}$ & $.13^{1}$ & $.08^{\mathrm{a}}$ & $.07^{1}$ \\
\hline ES & $.04^{\mathrm{a}}$ & $.16^{* 1,2}$ & $.07^{\mathrm{a}}$ & $.23 * * 1,2$ \\
\hline $\mathrm{Op}$ & $.06^{\mathrm{a}}$ & $.19 * * 1$ & $.12 * * \mathrm{a}$ & $.04^{2}$ \\
\hline \multicolumn{5}{|l|}{ Quadratic Slopes } \\
\hline Ex & $.01^{\mathrm{a}}$ & $.01 * * 1$ & $-.03 * b$ & $.01 *^{1}$ \\
\hline $\mathrm{Ag}$ & $.00^{\mathrm{c}}$ & $.02 * * * 1$ & $-.02 * b$ & $.00^{2}$ \\
\hline Co & $.01^{\mathrm{b}}$ & $.01^{1}$ & $.00^{\mathrm{b}}$ & $.00^{1}$ \\
\hline ES & $.02^{\mathrm{a}}$ & $.00^{2}$ & $-.01^{\mathrm{a}}$ & $.01 * * 1$ \\
\hline Op & $-.01^{b}$ & $.01 * * 1$ & $-.03 * * \mathrm{c}$ & $.00^{1}$ \\
\hline
\end{tabular}

Note. Intercepts were moved to age 16 (i.e., fifth measurement in the younger cohort, and first measurement in the older cohort). Different superscripts indicate significant $(p<.05)$ gender, age cohort, or gender $\mathrm{X}$ age cohort differences in means and variances of intercepts, linear slopes and quadratic slopes. To facilitate interpretation, comparisons between means are represented with letters, whereas comparisons between variances are represented with digits. ${ }^{*} p<.05, * * p<.01, * * * p<.001$. 

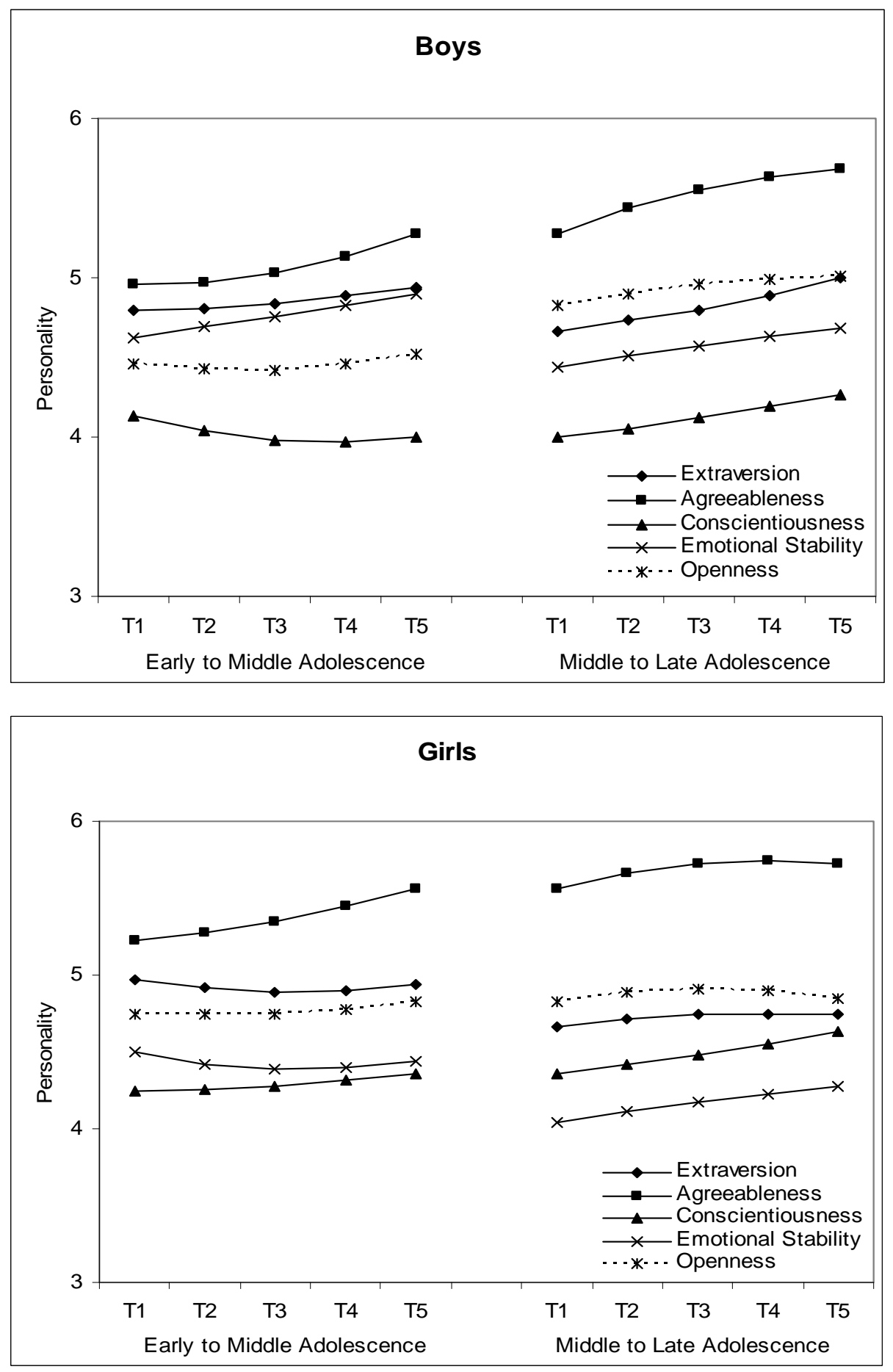

Figure 2.1. Estimated Growth of Extraversion, Agreeableness, Conscientiousness, Emotional Stability, and Openness for Boys and Girls, respectively.

For Emotional Stability, we generally found increases within cohorts. However, there were large cohort effects, as levels of Emotional Stability were much lower in middle to late adolescence, when compared to early to middle adolescence. In addition, there were clear gender differences, as girls had much lower levels of Emotional Stability than boys in early 
adolescence. These gender differences became even somewhat larger towards middle adolescence, as levels of Emotional Stability increased for boys, but remained stable for girls. However, these gender differences in change were just not significant. In the middle to late adolescent cohort, girls again had much lower levels of Emotional Stability than boys. As both boys and girls exhibited a similar increase, gender differences in Emotional Stability were maintained across time.

We only found some evidence for increases in Openness throughout adolescence. However, there were substantial gender differences and a cohort effect. From early adolescence to middle adolescence, girls displayed higher levels of Openness than boys, although boys displayed curvilinear increases, whereas girls retained a stable level of Openness. For boys, there was a cohort effect, as their levels of Openness were higher in middle to late adolescence than in early to middle adolescence, even though we compared the two age cohorts at a point where their mean ages were similar (i.e., 16 years old). For girls, such cohort differences were not found. As a result, boys and girls had similar levels of Openness in middle to late adolescence. In that period, girls displayed a curvilinear pattern which resulted in very little change across time. Changes for boys did not reach significance.

\section{Rank-order Stability}

Pearson correlations were calculated to assess rank-order stability of personality across adolescence. For boys, correlations ranged from .31 (Agreeableness from T1 to T2) to .62 (Conscientiousness from T3 to T4) in early to middle adolescence, and from .27 (Agreeableness from T1 to T2) to .75 (Conscientiousness from T3 to T4) in middle to late adolescence. Test-retest correlations for girls ranged from .41 (Agreeableness T1 to T2) to .75 (Extraversion T4 to T5) in early to middle adolescence, and from .52 (Agreeableness T1 to T2, and T3 to T4) to .86 (Conscientiousness T4 to T5) in middle to late adolescence. All testretest correlations for boys and girls are displayed in Table 2.4.

To test the hypothesis that rank-order stability would increase as adolescents grow older, differences between correlation coefficients in early to middle adolescence and middle to late adolescence were tested for significance $(p<.05)$ across all four between-measurement intervals (i.e., T1-T2, T2-T3, T3-T4, and T4-T5), for boys and girls separately. Significant differences between age cohorts are represented in bold type-font in Table 2.4.

For boys, test-retest correlations of Extraversion, Conscientiousness, and Emotional Stability were generally higher in middle to late adolescence when compared to early to middle adolescence, except for the T3-T4 correlations on all these three dimensions, which were 
equal in both age cohorts. In general, rank-order stability for Agreeableness and Openness did not increase with age, as only the T1-T2 correlation for Openness was significantly higher in the older cohort than in the younger cohort. In sum, ten out of twenty test-retest correlations were higher in the older cohort than in the younger cohort, the other ten test-retest correlations were not significantly different for the two age cohorts. Hence, we found evidence for agerelated increases in rank-order stability of personality traits for boys.

Table 2.4

Rank-order Stability and Profile Similarity of Personality in Early to Middle and Middle to Late Adolescent Boys and Girls

\begin{tabular}{|c|c|c|c|c|c|c|c|c|}
\hline & \multicolumn{4}{|c|}{ Early to Middle Adolescence } & \multicolumn{4}{|c|}{ Middle to Late Adolescence } \\
\hline & T1-T2 & T2-T3 & T3-T4 & T4-T5 & T1-T2 & T2-T3 & T3-T4 & T4-T5 \\
\hline \multicolumn{9}{|l|}{ Boys } \\
\hline Extraversion & .35 & $\underline{.48}$ & .62 & .60 & .59 & .65 & .71 & .75 \\
\hline Agreeableness & .31 & .48 & .53 & .47 & .27 & .42 & .48 & .34 \\
\hline Conscientiousness & .45 & .59 & .69 & .64 & .60 & .71 & .75 & .74 \\
\hline Emotional stability & .32 & .39 & .50 & .51 & .51 & .63 & .62 & .73 \\
\hline Openness & .36 & .51 & .61 & .61 & .53 & .58 & .62 & .70 \\
\hline Profile Similarity & .42 & .51 & .61 & .63 & .63 & .73 & .77 & .76 \\
\hline \multicolumn{9}{|l|}{ Girls } \\
\hline Extraversion & .55 & .67 & .68 & .75 & .70 & .76 & .81 & .82 \\
\hline Agreeableness & .41 & .46 & .51 & .53 & .52 & .56 & .52 & .67 \\
\hline Conscientiousness & .55 & .60 & .67 & .72 & .79 & .81 & .81 & .86 \\
\hline Emotional stability & .48 & .59 & .56 & .66 & .57 & .65 & .68 & .75 \\
\hline Openness & .52 & .54 & .60 & .69 & .64 & .69 & .67 & .79 \\
\hline Profile Similarity & .55 & .63 & .63 & .74 & .73 & .79 & .78 & .82 \\
\hline
\end{tabular}

Note. All correlations significant at $p<.001$. Underlined test-retest correlations represent significant gender differences. Test-retest correlations in bold face represent significant age differences.

For girls, test-retest correlations were generally higher in middle to late adolescence than in early to middle adolescence for Extraversion, Conscientiousness, and Openness (except for the T3-T4 correlation of Openness, which did not significantly differ for the two age cohorts). For Emotional Stability, test-retest correlations were equal for both age cohorts on the first two time intervals (T1-T2, and T2-T3), and were higher in the older cohort on the other two time intervals (T3-T4, and T4-T5). For Agreeableness, only the T3-T4 correlation was higher in the older cohort than in the younger cohort, the other test-retest correlations were not significantly different for the two age cohorts. Taken together, fourteen out of 


\section{Maturation of Personality}

twenty test-retest correlations were higher in middle to late adolescence than in early to middle adolescence. The remaining six test-retest correlations were equal in the two age cohorts. Therefore, evidence for age related increases in rank-order stability was even stronger in girls than in boys.

With regard to gender differences, eighteen out of forty test-retest correlations were significantly higher for girls than for boys. The other twenty-two were equal for both genders. Thus, girls generally exhibited higher levels of rank-order stability than boys. Test-retest correlations that were different for adolescent boys and girls are underlined in Table 2.4.

\section{Profile Similarity}

Profile similarity was measured by calculating $q$-correlations for all one-year betweenmeasurement intervals included in the study. Mean $q$-correlations are provided for early to middle and middle to late adolescent boys and girls, separately. This procedure resulted in a total of sixteen mean $q$-correlations, allowing us to examine increases in profile similarity through adolescence, and gender differences herein. Mean $q$-correlations for boys and girls are displayed in the bottom row of Table 2.4. For both boys and girls, average $q$-correlations were moderate to high in early to middle adolescence, and high in middle to late adolescence. We used multigroup LGCM to assess changes and gender differences in these changes for $q$ correlations. Growth parameters of the unconstrained model, and between-group differences as tested with Chi-square difference tests, are displayed in Table 2.5. Estimated growth of profile similarity in boys and girls is plotted in Figure 2.2.

The unconstrained model had an adequate fit $\left(\chi^{2}(12)=29.44(p=.003)\right.$; CFI = .97; $\mathrm{TLI}=.94 ; \mathrm{RMSEA}=.07(90 \%$ C.I. $=.04-.10))$, and revealed that profile similarity increased as adolescents grew older. Overall, increases were somewhat larger in early to middle adolescence than in middle to late adolescence. There were also large gender differences. Compared to boys, girls displayed higher levels of profile similarity in early adolescence. As a result, girls had already reached a mean $q$-correlation higher than .70 by middle adolescence. Boys on the other hand, displayed stronger curvilinear increases than girls, both in early to middle and middle to late adolescence. However, because they initially had much lower levels of profile similarity than girls, they had only reached an average mean $q$ correlation higher than .70 by late adolescence. 
Table 2.5

Growth Factors in the Unconstrained Multigroup Latent Growth Curve Model for Profile

Similarity in Early to Middle and Middle to Late Adolescent Boys and Girls

\begin{tabular}{|c|c|c|c|c|c|}
\hline \multirow[t]{2}{*}{ Growth Factors } & & \multicolumn{2}{|c|}{ Early to Middle Adolescents } & \multicolumn{2}{|c|}{ Middle to Late Adolescents } \\
\hline & & Boys & Girls & Boys & Girls \\
\hline \multirow[t]{2}{*}{ Intercepts } & Mean & $.42 * * * \mathrm{a}$ & $.56 * * * b$ & $.63 * * * b$ & $.75 * * * \mathrm{c}$ \\
\hline & $\sigma^{2}$ & $.14 * * * 1$ & $.10 * * * 1$ & $.11 * * * 1$ & $.01^{2}$ \\
\hline \multirow[t]{2}{*}{ Linear Slopes } & Mean & $.13 * * * \mathrm{a}$ & $.03^{\mathrm{b}}$ & $.12 * * * \mathrm{a}$ & $.03^{\mathrm{b}}$ \\
\hline & $\sigma^{2}$ & $.10 * 1$ & $.16^{* * * 2}$ & $.08 * 1$ & $.02^{3}$ \\
\hline \multirow[t]{2}{*}{ Quadratic Slopes } & Mean & $-.02 * \mathrm{a}$ & $.01^{\mathrm{b}}$ & $-.03 * * \mathrm{a}$ & $.00^{\mathrm{b}}$ \\
\hline & $\sigma^{2}$ & $.01^{1}$ & $.02 * * * 3$ & $.01 * * 2$ & $.00^{1}$ \\
\hline
\end{tabular}

Note. Different superscripts indicate significant $(p<.05)$ gender, age cohort, or gender $\mathrm{X}$ age cohort differences in means and variances of intercepts, linear slopes and quadratic slopes. To facilitate interpretation, comparisons between means are represented with letters, whereas comparisons between variances are represented with digits. $* p<.05, * * p<.01, * * * p<.001$.

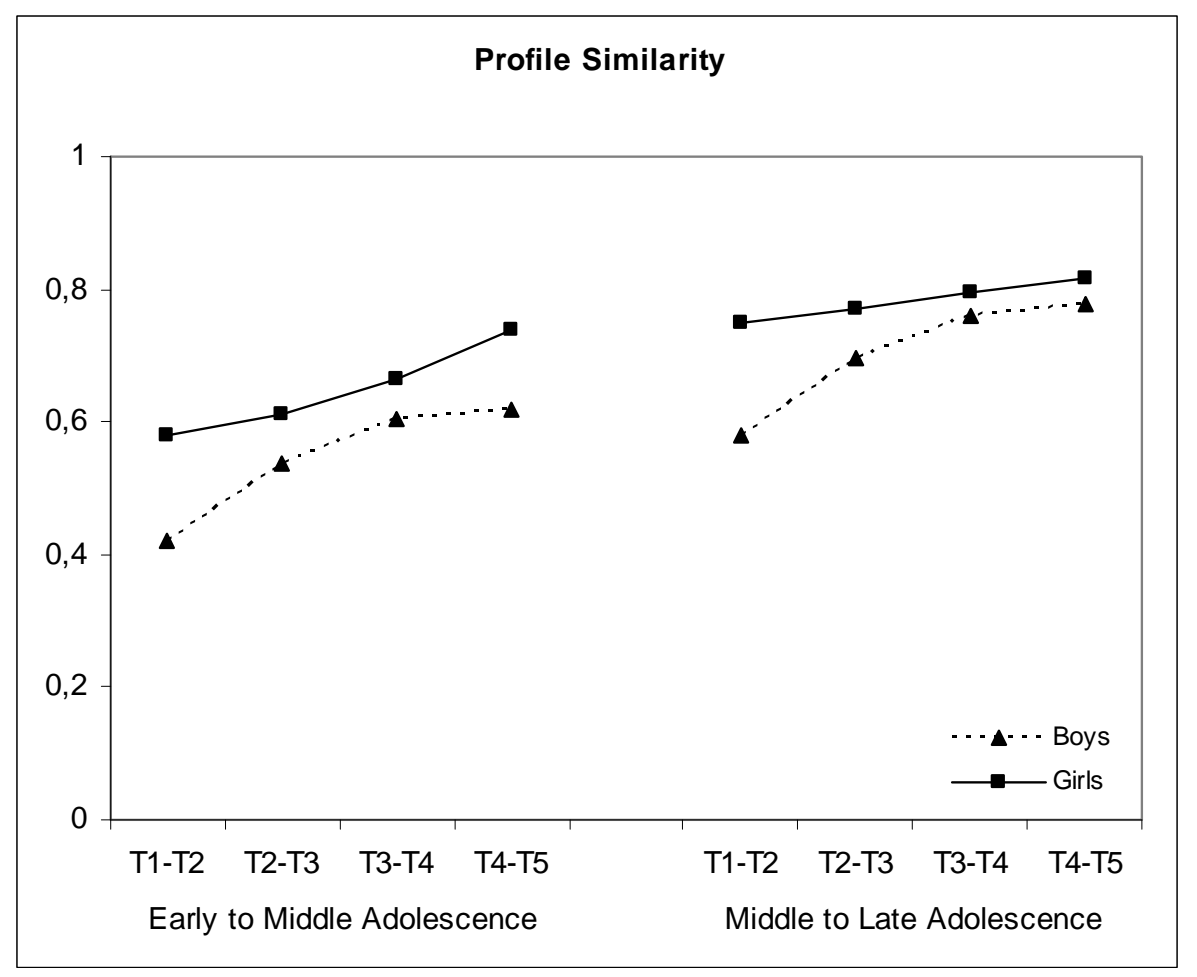

Figure 2.2.

Estimated growth of profile similarity ( $q$-correlations) for boys and girls. 


\subsection{Discussion}

In adolescence, the transition from being a dependent child towards becoming an independent adult takes place (Erikson, 1950). This maturation process should be reflected by mean-level increases on Big Five Traits (e.g., Caspi et al., 2005), increasingly more settled interindividual differences (e.g., Costa \& McCrae, 1994), and an increasingly better organized personality profile (e.g., Roberts et al., 2001). We assessed these three aspects of maturation using a five-year longitudinal design with overlapping early to middle and middle to late adolescent cohorts, and tested for gender differences. In general, we found evidence for all three aspects of maturation, and demonstrated that girls matured at a younger age than boys.

\section{General Changes in Mean-levels of Personality Traits}

With regard to mean-level changes, the strongest evidence for increases was found for Agreeableness. This was a surprising finding, considering that previous studies (Branje et al., 2007; De Fruyt et al., 2006; McCrae et al., 2002; Pullmann et al., 2006; Roberts et al., 2006) found very little evidence for maturation on this dimension We will discuss two possible causes for discrepancies between our results and those obtained with previous studies: (1) the effect of our inclusion of multiple measurement waves and (2) birth cohort differences between our sample and samples included in the meta-analysis by Roberts et al. (2006). In addition, a theoretical perspective on the increases in Agreeableness will be provided.

A first source of the discrepancies between our findings and those from previous studies is likely to be the inclusion of multiple measurement occasions across the entire range of adolescence. An examination of Figure $1 \mathrm{a}$ and $1 \mathrm{~b}$ reveals that there are indeed periods within adolescence where Agreeableness does not increase (e.g., T1-T3 in early to middle adolescent boys, and T2-T5 for middle to late adolescent girls). If we would have concentrated on these specific periods within adolescence, we would have found no change with regard to Agreeableness. However, when the entire range of adolescence is taken into account, mean-level increases can readily be observed. For that reason, our results demonstrate that, in order to obtain a comprehensive and reliable perspective on adolescent personality development, frequent assessments that cover the entire period of adolescence are needed.

Second, discrepancies between our findings and those obtained with a meta-analysis (Roberts et al., 2006) could be caused by birth cohort effects. Roberts et al. (2006) included 
cohorts that grew up in 1960s and 1970s, when questioning of norms and traditions was a widespread phenomenon. Roberts et al. (2006) noted that cohorts that grew up in that era, tended to exhibit little increases in Agreeableness. Cohorts born before and after that period, when norms and traditions were questioned to a lesser extend (see Roberts et al., 2006), displayed much larger increases. The fact that the cohorts included in the current study were born in the late 1980s, whereas the meta-analysis by Roberts et al. (2006) included cohorts growing up in the 1960s and 1970s, could explain why we found increases in Agreeableness, whereas such increases were not found in the meta-analysis.

Although the increases we found for Agreeableness may not be in agreement with findings from a majority of previous studies, they make theoretical sense. In adolescence, peer relations become increasingly more salient and intimate (e.g., Furman \& Buhrmester, 1992; Shulman, Laursen, Kalman, \& Karpovsky, 1997). In order to form more intimate relationships, it is important to be able to maintain positive relationships. The ability to maintain positive relationships with others has been shown to be related to Agreeableness (Jensen-Campbell et al., 2002), which could explain the mean-level increases for Agreeableness in the current study.

Similar to previous studies (De Fruyt et al., 2006; McCrae et al., 2002; Pullmann et al., 2006; Roberts et al., 2006), we found little evidence for increases in Conscientiousness. Conscientiousness comprises lower-order traits of self-control (Jensen-Campbell \& Malcolm, 2007), attention, persistency, and responsibility (Caspi et al., 2005). These lower order traits are important for keeping up a reasonable level of performance at school. Hence, higher levels of Conscientiousness have been shown to be related to academic achievement (Di Fabio \& Busoni, 2007; Graziano \& Ward, 1992; Noftle \& Robins, 2007). Academic achievement is important throughout the entire period of adolescence, and it is therefore perhaps not surprising that our results, as well as results of previous studies, indicate that levels of Conscientiousness are quite stable throughout adolescence.

In the current study, we found some evidence for small increases in Openness. These increases were consistent with a majority of previous studies, except for De Fruyt et al. (2006), who found decreases in early adolescence. Openness has been shown to be related to the most advanced status in identity formation, identity achievement (e.g., Crocetti, Rubini, Luyckx, \& Meeus, 2008; Luyckx, Goossens, Soenens, Beyers, \& Vansteenkiste, 2005). The achievement of a stable sense of identity is considered to be crucial to advance to subsequent developmental stages after adolescence (Erikson, 1950). In line with this reasoning, Openness has also been found to be beneficial during one major transition that awaits after adolescence: 


\section{Maturation of Personality}

the entrance into the labor market. Openness has been shown to be adaptive in the early stages of a professional career, as Openness is positively related to success in job interviews (Caldwell \& Burger, 1998), and training proficiency (Salgado, 1997). Increases in Openness could, therefore, indicate that adolescents become increasingly more ready for the developmental challenges that await after adolescence.

Similar to some previous studies (Pullmann et al., 2006; Roberts et al., 2006), we found some evidence for maturation within cohorts for Extraversion. Similar increases within age cohorts were found for Emotional Stability. These increases were in agreement with previous studies by De Fruyt et al. (2006), Pullmann et al. (2006), and Roberts et al. (2006). Surprisingly, the age-related increases for Extraversion and Emotional Stability in the current study were accompanied by counter-intuitive differences between cohorts. Levels of Extraversion and Emotional Stability were lower in middle to late adolescents than they were in early to middle adolescence. Cohort effects were not expected in general, as we compared levels of personality traits for the two age cohorts at the point where the two cohorts had experienced a similar amount of years of education, and had similar mean ages. In addition, we found no differences between the cohorts on background variables. Birth cohort effects have been reported before (Twenge, 2000, 2001), but were not expected to occur in this study as participants in the younger cohort were only born four years after the participants in the older cohort.

Despite cohort effects, the increases we found for Emotional Stability within the separate age cohorts suggest that adolescents cope with the stresses of life in an increasingly more adaptive way as they grow older. Similar to the increases in Emotional Stability, the small increases in Extraversion can also be interpreted as maturation. Extraversion comprises lower order traits of shyness, social competence, dominance, and activity level (Caspi et al., 2005). Therefore, the slight increases in Extraversion in the current study, signify decreases in shyness, and increases in social competence, dominance, and activity level. These changes should help adolescents to behave in a more mature way.

The evidence we found with regard to maturation can be explained in terms of social investment (Roberts \& Wood, 2006), as three of the Big Five traits have been shown to be related to social investment (i.e., Agreeableness, Conscientiousness, and Emotional Stability; Lodi-Smith \& Roberts, 2007), and another one has been hypothesized to indicate social investment (i.e., Extraversion; Roberts, Wood, \& Smith, 2005). Social investment refers to investment in, and commitment to adult social roles in the primary domains of work, family and society at large (Lodi-Smith \& Roberts, 2007). Concrete conceptualizations of these 
domains of social investment include career (i.e., work), social investment in a relation with a romantic partner and/or one's children (i.e., family), and volunteer work (i.e., society). Hence, the changes towards maturity we observed for Extraversion, Agreeableness, and Emotional Stability, could reflect a process wherein adolescents are becoming increasingly ready to socially invest as they grow older.

\section{General Changes in Stability of Personality}

Individual differences in Big Five personality traits became much more set with age in the current study. In early to middle adolescence, almost all test-retest correlations were already moderate (i.e., >.30), and they continued to increase in a systematic way in middle to late adolescence. In fact, a spectacular increase can be observed in Table 4, as average rankorder stability increases from .43 in early adolescence to .72 in late adolescence. Therefore, our study confirms the results from a meta-analysis that derived the Big Five factors from measures not specifically designed to measure these traits (Roberts \& DelVecchio, 2000), and two empirical studies that used a limited number of measurement occasions (Akse et al., 2007; Pullmann et al., 2006). The current study demonstrated that the increases in rank-order stability found in these previous studies, take place in a systematic way throughout adolescence. Hence, the view that individual differences in personality traits become increasingly more set with age (e.g., Costa \& McCrae, 1994) is strongly supported by the current study.

The profound increases in rank-order stability did not imply that mean-level changes no longer occurred. For example, mean-levels of Extraversion in girls changed significantly in middle to late adolescence, while rank-order stability was over .70 at that point. Thus, our results show that high rank-order stability can indeed be accompanied by mean-level changes, because these two change indices are independent from one another (Roberts \& DelVecchio, 2000; Roberts et al., 2006). If mean-levels continue to change, while the rank-order remains quite stable, most adolescents must change in the same direction and at a similar change rate. Changes that occur to a similar degree in most people in the population, are called normative changes, and reflect universal maturation processes (e.g., Helson, Kwan, John, \& Jones, 2002; Kasen, Chen, Sneed, Crawford, \& Cohen, 2006; Roberts \& DelVecchio, 2000; Roberts et al., 2006). Thus, as adolescents grow older, an increasing proportion of the mean-level changes that occur should be interpreted as normative changes.

Apart from evidence for maturation on separate personality traits, we also found strong evidence for increases in the stability of personality profiles. The formation of a stable 
personality profile is considered an important sign of maturation, as previous studies have shown that personality traits indicating maturity (i.e. constraint, negative and positive emotionality) predict profile similarity (Roberts et al., 2001; Donnelan, Conger, \& Burzette, 2007). The average stability of Big Five personality trait constellations was only moderate in early adolescence (.42 and .55 between $\mathrm{T} 1$ and $\mathrm{T} 2$ for early to middle adolescent boys and girls, respectively), but increased to a high level in late adolescence (.76 and .82 between T4 and T5 for middle to late adolescent boys and girls, respectively). Increases were much larger in early to middle adolescence than in middle to late adolescence. In late adolescence, profile similarity seems to have reached a plateau level, as substantial increases no longer occurred.

In sum, adolescent personality development in the current study is described by meanlevel increases on most personality traits, especially for Agreeableness and Emotional Stability, whereas there was also some evidence for increases in Extraversion and Openness. Furthermore, individual differences become much more settled with age, and personality profiles become better organized. There were, however, substantial gender differences for all these three indicators of adolescent personality maturation. These gender differences will now be discussed.

\section{Gender Differences in Adolescent Personality Maturation}

With regard to the first indicator of maturation, mean-level increases (e.g., Caspi et al., 2005), profound gender differences were found in the current study. These gender differences applied to initial levels, as well as change rates.

For Agreeableness, girls exhibited higher initial levels and increased at a faster rate than boys in early to middle adolescence. Only Branje et al. (2007) had similar findings, although they only found gender differences in change rates. Branje et al. (2007) already suggested that boys could catch up with girls with regard to personality development. They were unable to test this proposition, but our results for Agreeableness suggest that that boys indeed caught up with girls. Thus, our results suggest that girls mature at an earlier age than boys with regard to Agreeableness.

In early adolescence, girls also displayed higher levels of Conscientiousness than boys. These gender differences became larger towards middle adolescence, as boys displayed a small curvilinear decrease, whereas girls retained a stable level of Conscientiousness. In middle to late adolescence, the gender differences persisted, as both boys and girls displayed stable levels of Conscientiousness. Gender differences in mean-levels of Conscientiousness were not found in previous studies. However, Jensen-Campbell \& Malcolm (2007) showed 
that Conscientiousness is important for maintaining positive interpersonal relationships. We previously discussed the importance of Agreeableness with regard to that domain. Taken together, the gender differences in Agreeableness and Conscientiousness can be explained from an evolutionary point-of-view (Geary, Byrd-Craven, Hoard, Vigil, \& Numtee, 2003), as the maintenance of positive interpersonal relationships is much more emphasized by girls than by boys. Boys, on the other hand, are more focused on dominance in social relationships (Geary et al., 2003), which is among the lower order traits that comprise Extraversion (Caspi et al., 2005). However, this greater evolutionary importance of dominance for boys when compared to girls is not reflected by gender differences in Extraversion in the current study. Similar to previous studies by Pullmann et al. (2006) and McCrae et al. (2002) we found very little evidence for gender differences in adolescence.

In the current study, girls had higher levels of Openness in early adolescence to middle adolescence. McCrae et al. (2002) had previously demonstrated similar gender differences in mean-levels of Openness. Unlike boys, girls did display curvilinear changes in middle to late adolescence, but these changes were only small. Overall, boys and girls displayed similar levels of Openness in middle to late adolescence. However, our findings for Openness should be interpreted with some care, because we found a cohort effect for boys indicating that levels of Openness were higher in the older cohort than in the younger cohort. The possible causes of such cohort effects were discussed earlier, as we found similar cohort effects in general changes of Emotional Stability and Extraversion.

For Emotional Stability, our results were similar to those from studies by McCrae et al. (2002) and Pullmann et al. (2006), as we found a more advantageous developmental pattern for boys when compared to girls. Gender differences in Emotional Stability began to emerge towards middle adolescence, as boys increased and girls did not change. These gender differences persisted in middle to late adolescence, as boys and girls displayed similar change rates in the older cohort. The gender differences in Emotional Stability in the current study mirror the pattern of gender differences found in studies on internalizing problems (Clark, Smith, Neighbors, Skerlec, \& Randall, 1994; Hale, Raaijmakers, Muris, Van Hoof, \& Meeus, 2008), which consistently demonstrate higher levels of internalizing problems in girls when compared to boys.

Overall, the gender differences we found were not in agreement with findings from the meta-analysis by Roberts et al. (2006), as they did not find gender differences in mean-level change. The figures with the growth curves reveal a possible explanation for the discrepancies between our findings and theirs: by the end of adolescence most of the gender difference have 
disappeared. When Roberts et al. (2006) assessed mean-level changes across adolescence they were unable to assess gender differences in the timing of changes within adolescence. The contrast between the findings from their meta-analyses and our findings highlight the main contribution of our study with regard to gender differences in mean-level change: the most noticeable gender differences are not related to the magnitude of change across adolescence, it is the timing of these changes within adolescence that is different for boys and girls.

Gender differences for the second aspect of maturation, an increasingly more stable rank-order of individuals on personality traits (e.g., Costa \& McCrae, 1994), were even more clear than gender differences in mean-level change. Throughout adolescence, girls exhibited higher levels of rank-order stability than boys did. As a result, they had already reached very high levels of rank-order stability (i.e., >.70) by middle adolescence. Boys only reached such high levels of rank-order stability in late adolescence. However, girls were not only earlier with reaching high levels of rank-order stability. In fact, levels of rank-order stability continued to rise in late adolescence at a similar pace for boys and girls. As a result, gender differences were maintained across time.

With regard to the third aspect of maturation, increasingly more stable personality profiles (e.g., Roberts et al., 2001), there were again substantial gender differences. Girls had much more stable profiles when compared to boys in early to middle adolescence. By middle adolescence, girls had already reached high levels of profile similarity ( $>.70)$, whereas boys passed this .70 benchmark approximately 2 years later. Hence, girls have a stable personality at an earlier stage of adolescence than boys. Our findings with regard to the third aspect of maturation, an increasingly more stable personality profile, are therefore consistent with our findings on the first and second aspect of maturation.

In sum, our findings with regard to all three aspects of personality maturation (i.e., increasing mean-levels, increasingly more stable inter-individuals differences, and better organized personality profiles) provide evidence for maturation. In agreement with a suggestion by Branje et al. (2007), we found that gender differences in personality maturation often disappear in late adolescence. Hence, there is clear evidence for gender differences in the timing of personality maturation.

Coincidently, the age differences boys and girls display in reaching high levels of profile similarity and rank-order stability (approximately 2 years) is about the same as the age difference that has been found with regard to peak height velocity (i.e., the age at which an adolescent exhibits the fastest growth rate), which is an important marker of pubertal timing (e.g., Beunen et al., 2000; Petersen et al., 1988). Girls have also been shown to be ahead in 
brain development (Giedd et al., 1999; Lynn, 1994). These biological advantages seem to be reflected in personality development. However, to investigate whether Big Five personality development on the one hand, and biological or neurological development on the other hand are indeed related, future studies should assess both types of maturation in the same longitudinal design.

Girls not only have an advantage with regard to biological and neuronal development, they have additionally been found to exhibit higher levels of self-reflection than boys (Burwell \& Shirk, 2007). These gender differences in self-reflection could also explain gender differences in personality development. Repetitively reflecting on oneself could possibly facilitate personality consistency. However, self-reflection is also associated with rumination (Trapnell \& Campbell, 1999), which has been shown to be more prevalent among girls as well (Compas, Malcarne, \& Fondacero, 1988). Rumination is a strong predictor of internalizing problem behaviors, such as depression (for an overview, see: Hyde, Mezulis, \& Abramson, 2008). As a result, self-reflection might facilitate maturation of personality, but it can have a detrimental effect as well. In the current study, this harmful effect was possibly reflected in the disadvantageous developmental pattern girls displayed for Emotional Stability.

\section{Limitations and Future Directions}

Several limitations of the current study need to be recognized. First, we only used adolescent self-reports to assess personality traits. Social desirability might have a negative impact on the validity, and reported personality change might be caused by changes in beliefs adolescents have about their self (Robins, Fraley, Roberts, \& Trzesniewski, 2001). However, Robins, Noftle, Trzesniewski, and Roberts (2005) demonstrated that beliefs about changes in personality were actually quite accurate in a sample of young adults. In addition, Soto et al. (2008) recently showed that self-reports already provide an accurate estimate of personality at age ten, and that the psychometrics of the Big Five do not change as adolescents grow older. Therefore, we believe that our use of adolescent self-reports is an appropriate method to assess adolescent personality change.

A second potential limitation is that the current study only focuses on the patterns of personality trait development (i.e., the role of age in personality change), and the role of gender in these patterns. Several other factors, like family relations (Branje et al., 2004) and genetic factors (e.g., Caspi et al., 2005; De Fruyt et al., 2006) have been suggested as moderators of personality change. Even though our examination of the role of gender and the 
inclusion of multiple measurement waves already adds substantially to the understanding of adolescent personality development, the role of other potential moderators of adolescent personality change should also be assessed in future studies.

The present study assesses personality traits across shorter time intervals than most previous studies did, but the one-year interval between measurements can still be considered as a third limitation. In one year, a lot of events can occur, and these events could have a substantial impact on personality. Measuring short-term fluctuations in personality traits would allow for an investigation of the susceptibility of personality traits to life events, and could thereby add to the discussions on stability and change in adolescent personality development.

A possible fourth, methodological, limitation is related to the specification of our growth model. Recent methodological advances allow to use chronological age as input in LGCM (e.g., Mehta \& West, 2000), but we used grade level to specify growth processes. In life-span theory (e.g., Baltes, Reese, \& Lipsitt, 1980; Baltes, Staudinger, \& Lindenberger, 1999), both chronological age and grade level are considered to be part of the same set of developmental influences: normative age-graded influences. Adolescent at the same grade level experience the same social expectations and environmental demands (e.g., Petersen \& Crockett, 1985), which means that grade level is just as good an indicator of maturation as chronological age. Moreover, chronological age was largely confounded with grade level in the current study.

Finally, a fifth limitation concerns the use of two age cohorts to cover the entire range of adolescence. We tried to 'glue' the two age cohorts together, by putting the intercept of the growth models at the one measurement wave where the two cohorts overlapped (i.e., T5 of the younger cohort and $\mathrm{T} 1$ of the older cohort). With this approach, we found predominantly systematic increases in rank-order stability and profile similarity. For mean-level change, however, cohort differences occurred for Extraversion and Emotional Stability, and in Openness for boys. Therefore, the cohorts could not always be 'glued' together to obtain an accelerated model of personality development through adolescence, with regard to mean-level change. The best solution to this problem would be to have annual assessments on one cohort across the entire range of adolescence. However, because conducting a five-year longitudinal study while keeping attrition rates low already requires a substantial investment, following adolescents with frequent measurements across even longer periods of time is usually not feasible. A viable solution to the problem we experienced with regard to cohort differences in 
mean-levels of personality traits, would be to have a cohort-sequential design with more than one wave of overlap between cohorts (e.g., Branje et al., 2007).

\section{Conclusion}

Despite potential limitations, the improved research design and analytic approach of the current study adds substantially to the understanding of adolescent personality development. We found strong evidence of adolescent personality maturation for all three indicators, as mean-levels of Agreeableness and Emotional Stability increased, interindividual differences became more settled, and personality profiles became increasingly more stable as adolescents grew older. Mixed evidence was found for mean-level increases in Extraversion and Openness. In addition, we found strong evidence for gender differences in the timing of personality maturation. Girls reached high mean-levels of Agreeableness at an earlier stage of adolescence when compared to boys. Our results further indicated that interindividual differences and personality profiles stabilize at an earlier age for girls than for boys. 


\section{Chapter 3}

Longitudinal Associations Between Personality Traits and Problem Behavior Symptoms in Adolescence $^{3}$

${ }^{3}$ Klimstra, T. A., Akse, J., Hale, W. W., Raaijmakers, Q. A. W., \& Meeus, W. H. J. (in press). Longitudinal Associations Between Personality Traits and Problem Behavior Symptoms in Adolescence. Journal of Research in Personality. 


\begin{abstract}
In the current study, five annual wave longitudinal data were employed to examine the associations between Big Five personality traits and problem behavior (i.e., depression and aggression) in early to middle and middle to late adolescent boys and girls. Using crosslagged panel models, we simultaneously tested two competing hypothesis: (1) a vulnerability hypothesis stating that Big Five personality traits would affect problem behavior, and (2) a scar hypothesis asserting that problem behavior would affect Big Five personality traits. Results supported both hypotheses, as the effects between personality and problem behavior were bidirectional. These findings have important theoretical and clinical implications, as they suggest a transactional process.
\end{abstract}




\subsection{Introduction}

During the last decade the development of personality has been documented extensively (e.g., Ozer \& Benet-Martínez, 2006; Caspi, Roberts, \& Shiner, 2005). Especially adolescence appears to be a period of rapid changes in personality (Klimstra, Hale, Raaijmakers, Branje, \& Meeus, 2009). Unfortunately, little is known about the way a developing personality is related to development in other domains, such as problem behavior. Such knowledge would be valuable, as Tackett (2006) points out that it is important to understand the extent to which personality dimensions predispose individuals to future problem behaviors in order to develop effective, targeted prevention programs. Additionally, clarifying the associations between personality and problem behavior has important etiological implications that may serve to identify specific causal influences on the development of problem behavior. It would, for example, be valuable to examine whether specific personality traits could predict the development of only one type of problem behavior (e.g., internalizing problems) or multiple types of problem behaviors (e.g., internalizing and externalizing problems). Thus, one could test for multifinality (von Bertalanffy, 1968). More important for personality research is that one could test how core traits or basic tendencies (i.e., personality traits) affect, and are affected by surface traits or characteristic adaptations (e.g., problem behavior symptoms) (Asendorpf \& Van Aken, 2003; McCrae \& Costa, 1999). The basic idea behind the distinction between core traits or basic tendencies and surface traits or characteristic adaptations, is that the former (i.e., personality traits) are more stable than the latter (i.e., problem behavior symptoms), and that personality traits would therefore be better predictors of problem behavior symptoms than vice versa.

Thus, studying the influences between personality traits and problem behavior symptoms is important from a clinical and a theoretic point-of-view. Unfortunately, studies that address the longitudinal associations between children's and adolescents' personality traits and problem behavior symptoms are scarce. The current study attempts to provide more insight in the associations between personality and problem behavior, by employing five annual wave data of overlapping cohorts of early to middle and middle to late adolescents. Thereby, an age range from ages 12 to 20 is available.

In an overview, Tackett (2006) described various models explaining the associations between personality and problem behaviour. First, there is the scar or complication model, which proposes that problem behavior may cause changes in personality traits. An example of this model would be that repeatedly experiencing severe anxiety symptoms could lead to a 
decrease in one's levels of emotional stability. Second, the pathoplasty or exacerbation perspective suggests that personality traits may affect the way problem behavior manifests itself. In this context, high levels of behavioral inhibition (which are related to low levels of Emotional Stability and Extraversion; e.g., Smits \& Boeck, 2006) have been shown to be related to less severe manifestations and a better prognosis in children with conduct disorder (Kerr, Tremblay, Pagani, \& Vitaro, 1997). Third, the vulnerability or predisposition model hypothesizes that certain personality characteristics may put adolescents at risk to develop problem behavior. For example, individuals with very low levels of extraversion could be overly inhibited and might therefore be more prone to develop depressive symptoms. Fourth, the spectrum model proposes that personality traits and problem behavior may lie on a single continuum, with problem behavior being a more extreme manifestation of a certain personality trait. In one specific spectrum model (i.e., the tripartite model) it is stated that the relations between anxiety and depression on the one hand, and emotional stability on the other hand, are so pervasive that the constructs could share a common underlying factor (e.g., Clark, Watson, \& Mineka, 1994).

In the current study we examine a sample drawn from the general population in which very few individuals reach clinical levels of problem behavior. As such, our data are not particularly suitable for examining whether personality affects the way problem behavior manifests itself (i.e., the pathoplasty or exacerbation model). In addition, with regard to the spectrum model one should ideally search for a common causal factor in personality traits and problem behavior. An example of such a factor would be genetics. Since no such factors were included in our research design, we will focus on the two models that can be tested by directly exploring longitudinal associations s between personality and problem behavior, namely (1) the scar or complication model and (2) the vulnerability or predisposition model (Krueger \& Tackett, 2003; Tackett, 2006; Widiger, Verheul, \& van den Brink, 1999). Until now, these models find little empirical support in childhood and adolescence, which can be solely attributed to a clear lack of studies. Since it is suggested that more direct tests of these models will help elucidate a comprehensive approach to conceptualize the association between personality and psychopathology (Tackett, 2006), we will test both models simultaneously in this study.

There are many cross-sectional studies that have established associations between Big Five personality dimensions and problem behaviors. Extraversion, which can be conceptualized as a tendency to engage in social behaviors, and experience frequent positive moods (Caspi et al., 2005), has been found to be negatively associated with internalizing 
problems, such as social inhibition, anxiety and depression (Asendorpf, 2003; Ehrler, Evans, \& McGhee, 1999; Ozer \& Benet-Martínez, 2006; Van Leeuwen, Mervielde, De Clercq, \& De Fruyt, 2007). Agreeableness, indicative of an individual's sociability, empathy, and cooperativeness (Caspi et al., 2005), has been found to be negatively related to affective and anxiety disorders (Ehrler et al., 1999; Ferguson, 2000) and externalizing behaviors, such as conduct disorder, attention deficits, hyperactivity, aggressiveness, risk taking, criminal behavior and substance dependence (e.g., Asendorpf, 2003; Ehrler et al., 1999; Lounsbury, Sunstrom, Loveland, \& Gibson, 2003; Ozer \& Benet-Martínez, 2006; Paunonen, 1998). Conscientiousness, comprising organizational and motivational aspects of a person's behavior (Klimstra et al., 2009), was found to be negatively associated with externalizing behaviors, such as conduct disorder, substance abuse, antisocial and criminal behavior, attention deficits, hyperactivity, aggressiveness and impulsivity (e.g., Asendorpf, 2003; Caprara, Barbaranelli, \& Zimbardo, 1996; Lounsbury, Steel, Loveland, \& Gibson, 2004; Scher \& Osterman, 2002). Neuroticism (the opposite pole to Emotional Stability), which is indicative of individual differences in the ability to deal with negative emotions (Caspi et al., 2005), was found to be positively associated with internalizing problems, such as social inhibition, affective and anxiety disorders (e.g., Ehrler et al., 1999; Krueger, 1999; Muris, Meesters, \& Blijlevens, 2007; Van Leeuwen et al., 2007). Additional associations between these personality and problem behavior have been found, but there is no consensus on whether these associations are positive, negative, or absent. For example, for the fifth Big Five dimension, Openness (i.e., the way and individual seeks for deals with new information; Klimstra et al., 2009), no consistent evidence with regard to associations with problem behavior has been provided by previous research.

Although the mentioned cross-sectional studies provide some insight into the associations between personality and problem behavior, they are not informative on the direction of effects between personality and problem behavior. As such, they do not provide evidence for the scar or the vulnerability model. Findings from the few longitudinal studies that are available can be described in accordance with these models. Several studies (Huey Jr. \& Weisz, 1997; Van Leeuwen, Mervielde, Braet, \& Bosmans, 2004) demonstrated that Extraversion and Emotional Stability are negative predictors of internalizing problem behavior. All these studies also found that Agreeableness was a negative predictor of externalizing problem behaviors. In addition, Van Leeuwen et al. (2004) showed that Conscientiousness was a negative predictor of externalizing problem behavior, while Huey Jr. and Weisz (1997) found Extraversion to positively predict externalizing problems. However, the studies by Huey Jr. 
and Weisz (1997), and Van Leeuwen et al. (2004) do not appear to control for initial levels of problem behavior.

Obviously, these findings are in line with the vulnerability model. Some recent personcentered studies also reported findings in line with this model, as adolescents who were lower on Extraversion were more at risk for developing a depressive mood (Akse, Hale, Engels, Raaijmakers, \& Meeus, 2004, 2007a; Dubas, Gerris, Janssens, \& Vermulst, 2002; Van Aken, Van Lieshout, Scholte, \& Haselager, 2002). In addition, adolescents who changed from an introverted and socially withdrawn personality type to a personality type that was more extraverted and less socially withdrawn, displayed decreasing levels of internalizing problem behavior, while increasing levels of internalizing problem behavior accompanied personality type changes in the opposite direction (Akse, Hale, Engels, Raaijmakers, \& Meeus, 2007b).

Conversely, some studies were in favor of the scar model. Most of these studies showed that internalizing and externalizing problem behavior in childhood predicted personality disorders in adulthood (e.g., Kasen et al., 2001), but very few have examined whether problem behavior predicts normal personality traits. Shiner, Masten \& Tellegen (2002) showed that higher levels of childhood adaptation (i.e., academic achievement, rule-abiding conduct, and social competence) predicted lower levels of the Multidimensional Personality Questionnaire (MPQ) superfactor negative emotionality in emerging adulthood, even after controlling for childhood levels of negative emotionality. In another study, Ge and Conger (1999) found that psychological distress experienced during the adolescent years $(7$ th -10 th grades) was related to the MPQ superfactors of negative and positive emotionality during the final year of high school (12th grade), whereas delinquency and substance use problems experienced during the early adolescent years were primarily related to the MPQ superfactor of constraint during the final year of high school, also after controlling for initial levels of personality.

Thus, there are some studies that have examined whether personality predicts problem behavior, and there are some studies that assessed if problem behavior could predict personality. Although there are studies that have examined effects of personality traits on DSM-IV axis II personality disorders and vice versa (e.g., Warner et al., 2004), there are to our knowledge no studies in which it was examined whether adolescent personality dimensions predicted DSM-IV axis I problem behavior symptoms and vice versa. Thus, for DSM-IV axis I symptomatology, the vulnerability hypothesis (i.e., the influence of personality on problem behavior) and the scar hypothesis (i.e., the influence of problem behavior on personality; e.g., Krueger \& Tackett, 2003; Nigg, 2006; Shiner \& Caspi, 2003; 
Widiger \& Trull, 1992) have, to our knowledge, not been tested simultaneously in the same design.

As we previously noted, problem behavior symptoms have been described as being less stable then personality traits (e.g., McCrae \& Costa, 1999). However, problem behavior symptoms (e.g., depressive symptoms) can be highly stable across time and may therefore not be susceptible to influences from other factors (Cole, Nolen-Hoeksema, Girgen, \& Paul, 2006). In other words, highly stable concepts are less likely to be affected by other factors. As such, it is important to compare the stability of problem behavior symptoms and personality traits when examining the reciprocal influences between these two constructs.

A way to accomplish this is to use multi-wave cross-lagged panel models (Burkholder \& Harlow, 2003) in which all concurrent, stability and prediction associations within and between concepts are estimated simultaneously over time. As such, multi-wave cross-lagged panel models are highly appropriate for disentangling the effects of personality on problem behavior and vice versa (e.g., Lehnart \& Neyer, 2006; Neyer \& Asendorpf, 2001). To our knowledge, these models have so far not been used to study the longitudinal associations between the Big Five personality dimensions and internalizing and externalizing problem behaviors in adolescence.

As such, the main goal of this study is to investigate the longitudinal predictions from personality dimensions to problem behaviors and vice versa using multi-wave cross-lagged panel models. It is unclear to what extent findings obtained in studies on unidirectional effects from personality to problem behavior (Huey Jr. \& Weisz, 1997; Van Leeuwen et al., 2004) and problem behavior to personality (Ge \& Conger, 1999; Shiner et al., 2002) can be generalized to our study on the bidirectional effects between these two constructs, especially because these previous studies employed analyses that do not control for the initial associations between personality and problem behavior. However, the cross-lagged panel models used in the current study do control for such associations. Despite these differences in the design of the current study and designs of previous studies, we still can derive some tentative hypotheses from those previous studies. Thus, we expect Extraversion to negatively predict internalizing problems and positively predict externalizing problems, Agreeableness and Conscientiousness to negatively predict externalizing problems and Emotional Stability to negatively predict internalizing problems over time. Because Ge and Conger (1999) and Shiner et al. (2002) did not use Big Five personality dimensions, we are unable to provide predictions with regard to the effect of problem behavior on specific Big Five personality dimensions. However, we do expect problem behavior to affect at least some of these 
dimensions. Previous studies only controlled for gender differences (Huey Jr. \& Weisz, 1997; Van Leeuwen et al., 2004) or assessed gender differences in personality dimensions different from the Big Five (Ge \& Conger, 1999). For that reason, we will also explore gender differences in the associations between Big Five personality dimensions and problem behavior.

\subsection{Method}

\section{Participants}

For this study, we used the first five annual waves of data from an ongoing longitudinal research project on Conflict And Management Of Relationships (CONAMORE; Meeus et al., 2006). The longitudinal sample consisted of 1,313 participants divided into an early to middle adolescent cohort $(n=923 ; 70.3 \%)$ who were 12.4 years of age on average $(S D=.59)$, and a middle to late adolescent cohort $(n=390 ; 29.7 \%)$ with an average age of 16.7 years $(S D=.80)$ during the first wave of measurement. The early to middle adolescent cohort consisted of 468 boys (50.7\%) and 455 girls (49.3\%), and the middle to late adolescent cohort consisted of 169 boys (43.3\%) and 221 girls (56.7\%). Because both age groups were assessed during five measurement waves, a total age range from 12 to 20 years was available.

Sample attrition was $1.2 \%$ across waves: in waves $1,2,3,4$, and 5 the number of participants was 1,313, 1,313, 1,293, 1,292 and 1,275, respectively. Missing values were estimated in SPSS, using the EM-procedure. Across waves $4.37 \%$ of the data was missing. Little's Missing Completely At Random Test (Little, 1988) revealed a normed $\chi^{2}\left(\chi^{2} / \mathrm{df}\right)$ of 1.22 which according to guidelines by Bollen (1989) indicates a good fit between sample scores with and without imputation.

Before initiating the administration of the questionnaires, both students and their parents received written information describing the aims of the study and, if the students decided to participate, they were required to provide written informed consent. Less than $1 \%$ decided not to participate. Written informed consent from the participating schools was also obtained. Students filled out the questionnaires anonymously, either in their own home or at their own high school. Verbal and written instructions about the questionnaires were provided.

\section{Measures}


Personality. The personality dimensions Extraversion, Agreeableness, Conscientiousness, Emotional Stability and Openness to Experience were measured using the shortened Dutch version of the Big Five questionnaire (Gerris et al., 1998; Goldberg, 1992). This questionnaire contains 30 items, such as: talkative (Extraversion), sympathetic (Agreeableness), systematic (Conscientiousness), nervous (Emotional Stability) and creative (Openness to Experience). The adolescents judged whether the 30 items applied to themselves on a 7-point scale, ranging from 'absolutely agree' to 'absolutely disagree'. Internal consistencies are high with mean alphas of .83 (range: .79 - .87) for Extraversion, .85 (range: .81 - .88) for Agreeableness, .86 (range: .84 - .89) for Conscientiousness, .82 (range: .81 - .83) for Emotional Stability and .78 (range: .77 - .79) for Openness to Experience over the five waves. Aggression. The aggression questionnaire of Björkqvist et al. (1992) is a self-report questionnaire. Reliability and construct validity have been shown to be strong in adolescent samples (e.g., Hale, Vandervalk, Akse, \& Meeus, 2008). The questionnaire consists of the subscales direct aggression, indirect aggression and withdrawal, but in this study only direct aggression is taken into account. This subscale contains 5 items. Sample items for direct aggression include: When I'm mad at a classmate, I will 'use abusive language about him/her in every situation', 'hit or kick the other' and 'try to trip him/her up'. The items are scored on a 4-point scale, ranging from 'never', 'sometimes', 'often' to 'very often'. In this study the Cronbach alphas for the direct aggression subscale were $.87 / .84 / .83 / .83 / .84$ for each wave respectively.

Depression. The Children's Depression Inventory (CDI; Kovacs, 1985) is a self-report questionnaire that captures meaningful variance in depressive symptoms for children and adolescents who either do or do not meet diagnostic criteria for the disorder. This scale has demonstrated convergent and discriminant validity, good internal consistency and adequate test-retest reliability in previous studies (e.g., Craighead, Smucker, Craighead, \& Ilardi, 1998; Hodges, 1990). The CDI consists of 27 items; sample questions include 'I'm sad all the time', 'It will never end-up right for me' and 'I do everything wrong'. The items are scored on a 3point scale, ranging from 'false', 'a bit true' to 'very true'. In this study the Cronbach alphas for the CDI were $.93 / .90 / .91 / .90 / .89$ for each wave respectively.

\section{Strategy of Analyses}

Longitudinal associations between Big Five traits, and aggression and depression were assessed with multigroup cross-lagged panel models by means of structural equation modeling in Mplus 5 (Muthén \& Muthén, 2007). Maximum Likelihood Robust (MLR) 
estimation was used, as MLR has been shown to be the most accurate estimator when the distribution of scores deviates from a normal distribution (Satorra \& Bentler, 1994), which turned out to be the case for the scores on our aggression and depression measures. The groups we distinguished were early to middle adolescent boys $(N=468)$, middle to late adolescent boys $(N=169)$, early to middle adolescent girls $(N=455)$, and middle to late adolescent girls $(N=221)$. Model fit was judged by assessing RMSEA's and CFI's. RMSEA's below .08, and CFI's over .95 indicate adequate model fit; relatively lower RMSEA's, and higher CFI's indicate a better fit when comparing models (Kline, 1998).

A sample cross-lagged panel model is depicted in Figure 3.1. As Figure 3.1 suggests, our cross-lagged panel models contain one-year stability paths (i.e., paths a1 to a4 for personality, and paths $\mathrm{b} 1$ to $\mathrm{b} 4$ for problem behavior), two-year stability paths (i.e., paths aa1 to aa3 for personality, and paths bb1 to bb3 for problem behavior), concurrent correlations (i.e., association cc), associations depicting correlated change (i.e., associations c2 to c5; see for example Neyer and Asendorpf, 2001 for why concurrent correlations on $\mathrm{t} 2$ and onwards should be interpreted as correlated change in cross-lagged path models), and cross-paths (i.e., paths $\mathrm{d} 1$ to $\mathrm{d} 4$ indicating the effect of personality on problem behavior, and paths e 1 to e 4 indicating effects of problem behavior on personality). The two-year stability paths were added for Big Five personality dimensions and problem behavior symptoms (i.e., T1 problem behavior -> T3 problem behavior; T2 problem behavior -> T4 problem behavior; T3 problem behavior $->$ T5 problem behavior) to improve model fit. This procedure, which leads to smaller one-year stability paths, has also been employed in previous studies (e.g., Cui, Donnelan, \& Conger, 2007).

In a first step, we tested whether constraining equivalent paths and correlations (i.e., those parameters indicated with the same letters in Figure 3.1) within groups (i.e., early to middle adolescent boys, early to middle adolescent girls, middle to late adolescent boys, and middle to late adolescent girls) had a negative impact on model fit. Thus, we compared several nested models: more parsimonious or constrained models with as much constrained parameters as possible and a less parsimonious model with all parameters freely estimated. For such comparisons, the use of multiple criteria has been advocated by Vandenberg and Lance (2000). We relied on three criteria to compare nested models: a significant chi-square difference test (Steiger, Shapiro, \& Browne, 1985), a difference in CFI of $>.01$ (Cheung \& Rensvold, 2002), and a difference in RMSEA of >.01 (Chen, 2007). Only if two of these criteria were satisfied, we would favor the less parsimonious and less constrained model over the more parsimonious model with additional constraints. 


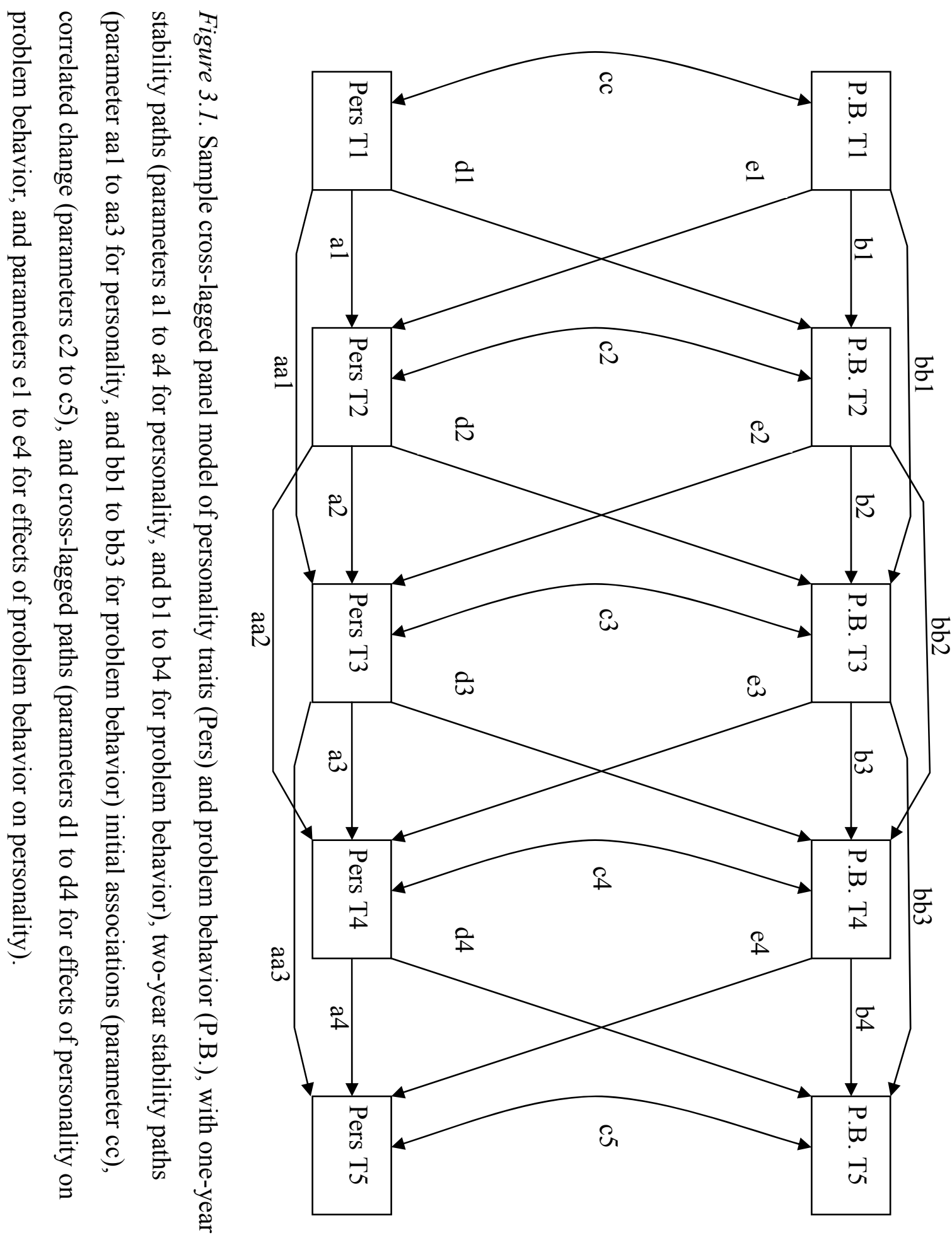


In a subsequent step, we tested for age cohort and gender cohort differences in parameter estimates. For this purpose, we compared models in which parameter estimates for early to middle adolescents and middle to late adolescents were freely estimated with more parsimonious models in which these estimates were constrained to be equal in the two age cohorts. Gender differences were tested in a similar way. That is, we compared models in which parameter estimates for boys and girls were freely estimated to models in which these estimates were constrained to be equal for boys and girls. For these model comparisons, we again used $\triangle \mathrm{CFI}, \triangle \mathrm{RMSEA}$, and chi-square difference tests.

In a last step, we examined whether personality was a better predictor for problem behavior, or whether problem behavior was a better predictor for personality. For that purpose, we compared models in which cross-paths from personality to problem behavior (d1 to $\mathrm{d} 4$ in Figure 3.1) and cross-paths from problem behavior to personality (e1 to e4 in Figure 3.1) were freely estimated, to more parsimonious models in which these paths were constrained to be equal to one another (i.e., $\mathrm{d} 1$ through $\mathrm{d} 4$ were constrained to be equal to e 1 through e4).

\subsection{Results}

In the cross-lagged panel models we used to calculate the longitudinal associations between personality and problem behavior, five annual waves of longitudinal data on early to middle and middle to late adolescent boys and girls were employed. Means and standard deviations of personality and problem behavior are depicted in Table 3.1.

Ten sets of cross-lagged path models were run to determine the longitudinal associations of problem behavior (i.e., depression and aggression) on the one hand and each of the Big Five traits on the other hand. For all models of the Big Five dimensions and problem behavior, correlated change coefficients, cross-paths from personality to problem behavior, and cross-paths from problem behavior to personality were constrained to be equal across measurement waves within groups (i.e., early to middle adolescent boys, early to middle adolescent girls, middle to late adolescent boys, and middle to late adolescent girls) because at least two out of three fit criteria indicated that adding such constraints did not result in a worse model fit ( $\triangle$ CFI's ranged from -.003 to .007 , indicating an insignificant improvement (for negative values) or deterioration (for positive values) of model fit, $\triangle$ RMSEA's ranged from .000 to -.015 indicating no change (if $\triangle \mathrm{RMSEA}>-.010$ ) or an significant improvement (if $\triangle$ RMSEA $<-.010$ ) of model fit). In addition, cross-paths, concurrent correlations, and 
Chapter 3

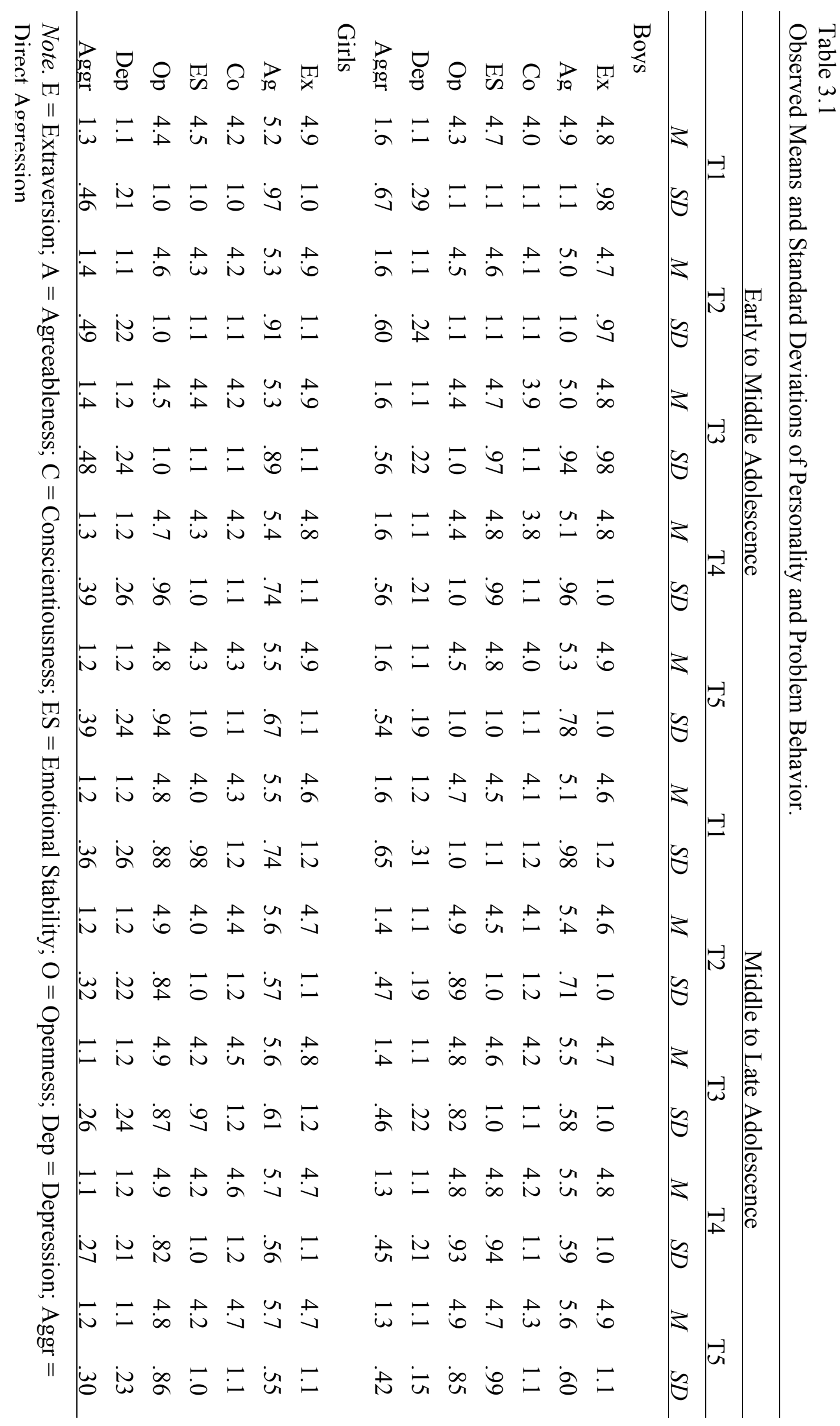


correlated change, were constrained to be equal across gender and age cohorts (i.e., early to middle adolescent boys, early to middle adolescent girls, middle to late adolescent boys, and middle to late adolescent girls) as at least two out of three fit criteria indicated that adding such constraints did not affect model fit ( $\Delta$ CFI's ranged from -.001 to .010 , indicating an insignificant improvement (for negative values) or deterioration (for positive values) of model fit, $\triangle$ RMSEA's ranged from -.004 to .005 indicating an insignificant improvement (for negative values) or deterioration (for positive values) of model fit). Even though the longitudinal associations were not different across measurement waves or for age and gender cohorts, at least two of the three criteria for measuring differences in model fit indicated that the stability paths for personality and problem behavior were different across time within gender and age cohorts in 50\% of the models (with regard to these model comparisons, all chi-square difference tests were significant $(p<.05)$ and $\Delta$ CFI's were $>.010$ (range: .011.012), both indicating a significant deterioration of model fit). For reasons of consistency, we decided to not constrain stability paths to be equal across time in any of the models. In addition, stability paths were also different for either the age cohorts (i.e., for the aggression models; all chi-square difference tests were significant $(p<.05)$ and $\Delta$ CFI's were $>.010$ (range: .014-.019), both indicating a significant deterioration of model fit) or gender cohorts (i.e., for the depression models; all chi-square difference tests were significant $(p<.05)$ and $\Delta$ CFI's were $>.010$ (range: .024-.043), both indicating a significant deterioration of model fit). More specifically, stability of middle to late adolescents was higher in the aggression models, whereas girls displayed higher levels of stability in the depression models. As a result, stability paths were only constrained to be equal across age cohorts in the depression models, and across gender cohorts in the aggression models. The fit statistics of the resulting models were adequate, and are depicted in Table 3.2.

The models will now be discussed for each Big Five dimension. We will first compare the stability of Big Five personality traits and problem behavior (i.e., Depression and Aggression). Second, we will pursue our primary research goal by examining the associations between Big Five traits and problem behavior. 
Table 3.2

Fit Statistic of the Final Cross-Lagged Panel Models

\begin{tabular}{lcccc}
\hline & $\chi^{2}$ & $d f$ & CFI & RMSEA \\
\hline Depression and Extraversion & $238.184^{* * *}$ & 148 & .973 & .049 \\
Depression and Agreeableness & $240.008^{* * *}$ & 148 & .955 & .049 \\
Depression and Conscientiousness & $257.381^{* * *}$ & 148 & .969 & .053 \\
Depression and Emotional Stability & $253.549^{* * *}$ & 148 & .964 & .052 \\
Depression and Openness & $238.364^{* * *}$ & 148 & .968 & .049 \\
Aggression and Extraversion & $222.035^{* * *}$ & 148 & .978 & .044 \\
Aggression and Agreeableness & $224.704^{* * *}$ & 148 & .963 & .045 \\
Aggression and Conscientiousness & $188.733^{*}$ & 148 & .989 & .033 \\
Aggression and Emotional Stability & $240.770^{* * *}$ & 148 & .967 & .049 \\
Aggression and Openness & $180.098^{*}$ & 148 & .989 & .029 \\
\hline
\end{tabular}

Note. ${ }^{* * *} p<.001 .{ }^{* *} p<.01 .{ }^{*} p<.05$.

\section{Stability of Personality Traits and Depression}

In the models concerning the associations between personality and depression, the stability paths were different for girls and boys. Therefore, we will discuss comparisons between the stability of specific personality traits and depression for boys and girls, separately.

As Table 3.3 suggests, chi-square difference tests indicated that depressive symptoms were less stable across time then Extraversion, Conscientiousness, and Openness for boys $(p<.05)$. However, depressive symptoms were equally stable when compared to Agreeableness and Emotional Stability.

Chi-square difference tests indicated that depressive symptoms were equally stable then four of the Big Five traits (i.e., Extraversion, Conscientiousness, Emotional Stability and Openness) in girls (see Table 3.4 for the parameter estimates). The fifth trait, Agreeableness, was less stable across time then depressive symptoms. 


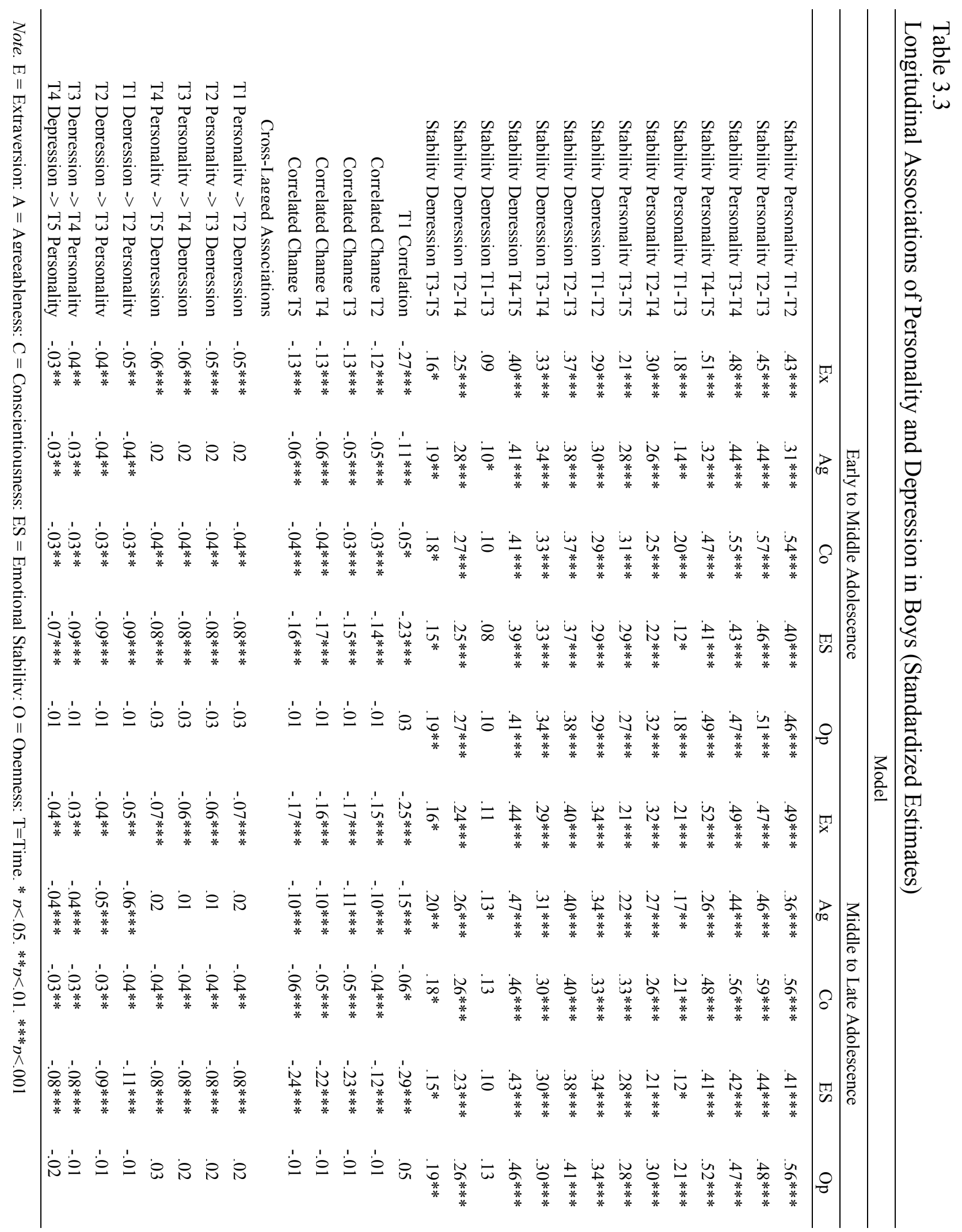




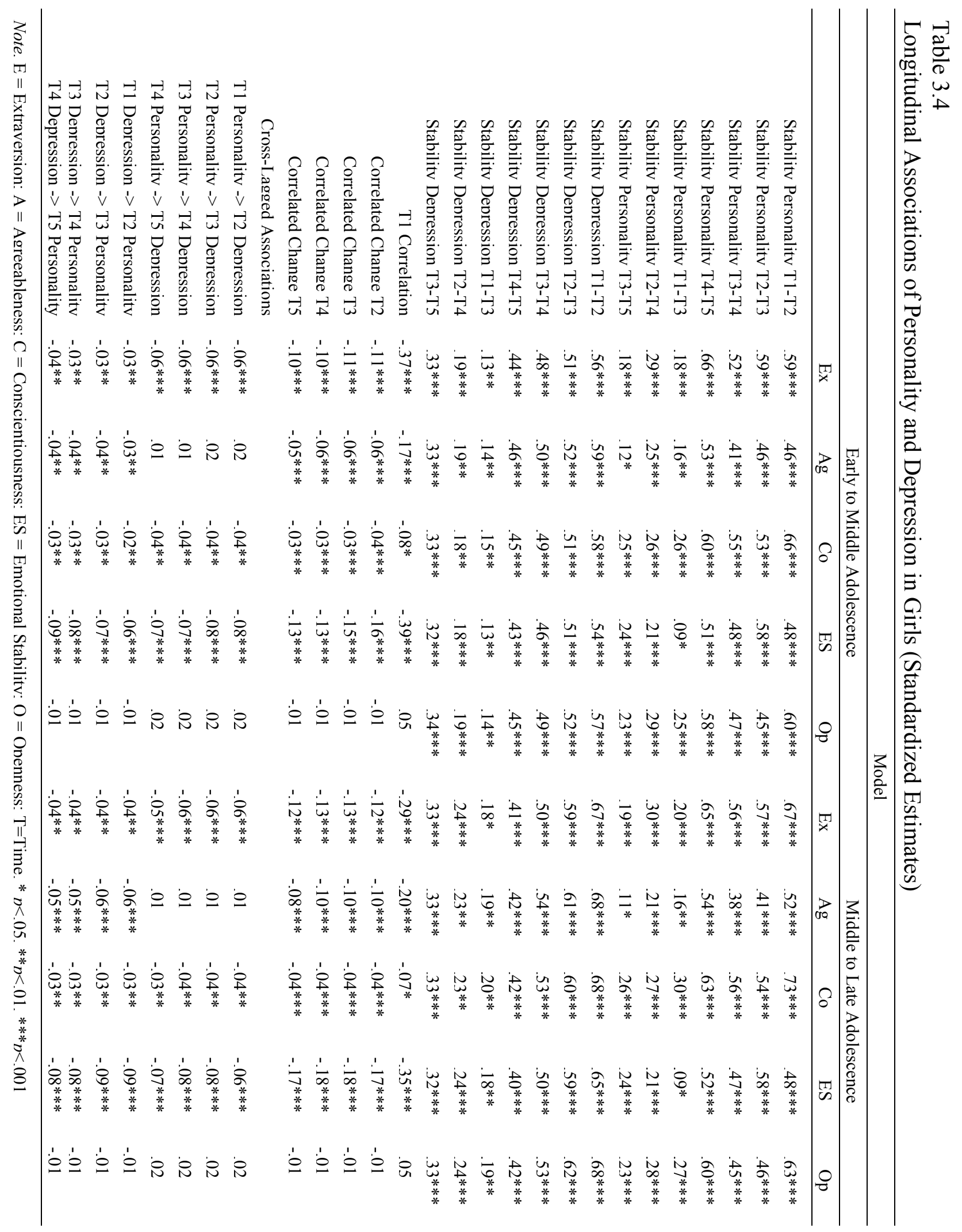


Personality and Problem Behavior

\section{Longitudinal Associations Between Personality and Depression}

Because longitudinal associations between personality and depression were constrained to be equal across gender and age cohorts, we are able to discuss these findings for all cohorts together.

Extraversion and Depression. T1 levels of Depression were negatively associated with T1 levels of Extraversion, and changes in Depression were negatively associated with changes in Extraversion (see Tables 3.3 and 3.4 for parameter estimates of boys and girls, respectively). Finally, high levels of Extraversion were predictive of relatively lower levels of Depression, and high levels of Depression predicted lower levels of Extraversion.

Agreeableness and Depression. Initial levels and changes in Agreeableness and Depression were negatively associated with one another (see Table 3.3 and Table 3.4). Significant cross-paths from Depression to Agreeableness indicated that high levels of Depression predicted relatively lower levels of Agreeableness. Cross-paths from Agreeableness to Depression did not reach significance.

Conscientiousness and Depression. Initial levels and changes in Conscientiousness and Depression were negatively associated with one another (see Tables 3.3 and 3.4). Significant cross-paths indicated that higher levels of Conscientiousness were predictive of relatively lower levels of Depression, whereas higher levels of Depression were predictive of lower levels of Conscientiousness.

Emotional Stability and Depression. Both initial levels and changes in Emotional Stability and Depression were negatively associated with one another (see Tables 3.3 and 3.4). Significant cross-paths from Emotional Stability to Depression indicated that higher levels of Emotional stability predicted relatively lower levels of Depression. Cross-paths in the inverse direction also reached significance and indicated that high levels of Depression predicted relatively lower levels of Emotional Stability.

Openness and Depression. Initial levels and changes in Depression and Openness were not associated with one another (see Tables 3.3 and 3.4). In addition, none of the cross-paths between Depression and Openness reached significance.

\section{Stability of Personality and Aggression}

In the models involving the associations between personality and aggression, the stability paths were different for the two age cohorts (i.e., early to middle adolescents and middle to late adolescents). Therefore, we will discuss comparisons between the stability of specific 
personality traits and aggression for early to middle adolescents and middle to late adolescents, separately.

For early to middle adolescents, chi-square difference tests revealed that aggression was equally stable as the Big Five personality traits (see Table 3.5 and 3.6 for parameter estimates). Chi-square difference tests revealed that four of the Big Five personality traits were more stable across time then aggression for middle to late adolescents $(p<.05)$. Agreeableness was equally stable as aggression.

\section{Longitudinal Associations Between Personality and Aggression}

Extraversion and Aggression. Initial levels and changes in Extraversion and Aggression were not associated. Furthermore, none of the cross-paths reached significance (see Table 3.5 for boys' parameter estimates, and Table 3.6 for girls' parameter estimates).

Agreeableness and Aggression. Initial levels and changes of Aggression and Agreeableness were negatively associated with one another (see Tables 3.5 and 3.6). Significant cross-paths from Agreeableness to Aggression indicated that higher levels of Agreeableness were predictive of lower levels of Aggression, and significant cross-paths in the inverse direction indicated that higher levels of Aggression predicted lower levels of Agreeableness.

Conscientiousness and Aggression. Initial levels and changes in Conscientiousness were negatively associated with initial levels and changes in Aggression (see Tables 3.5 and 3.6). Furthermore, significant cross-paths from Conscientiousness to Aggression indicated that higher levels of Conscientiousness predicted relatively lower levels of Aggression. Crosspaths in the inverse direction (i.e., from Aggression to Conscientiousness) were not significant.

Emotional Stability and Aggression. Initial levels of Emotional Stability and Aggression were negatively associated with one another, just like changes in these two variables (see Table 3.5 and 3.6). Furthermore, significant cross-paths indicated that high levels of Aggression predicted relatively lower levels of Emotional Stability. Cross-paths from Emotional Stability to Aggression did not reach significance.

Openness and Aggression. Initial levels of Openness and Aggression were not associated with one another, but changes in Openness and Aggression were found to be negatively associated (see Tables 3.5 and 3.6). In addition, significant cross-paths indicated that higher levels of Openness predicted relatively lower levels of Aggression. Cross-paths from Aggression to Openness did not reach significance. 
Personality and Problem Behavior

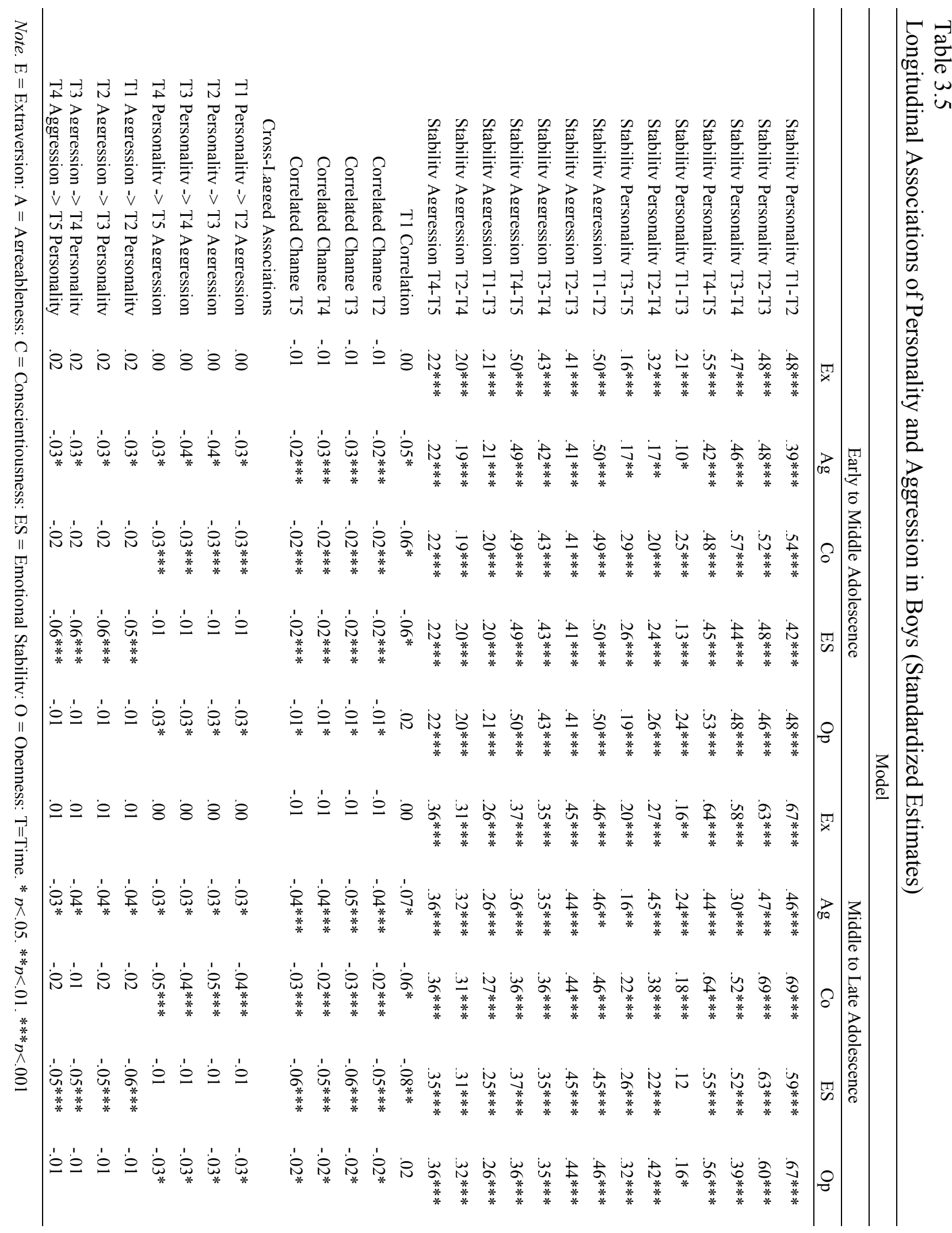




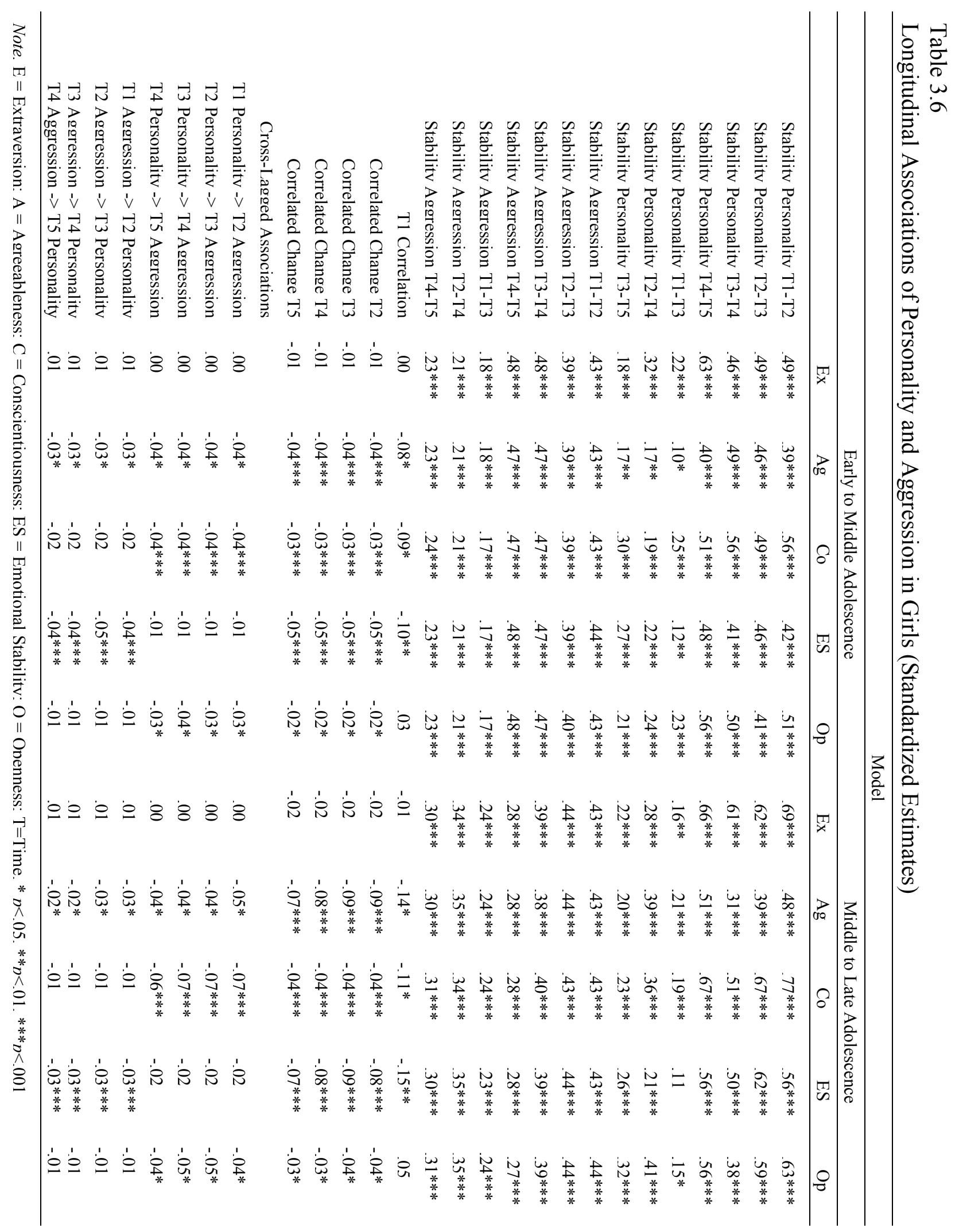




\section{Head-to-Head Comparisons of the Predictive Properties of Personality and Problem}

\section{Behavior.}

In a subsequent step, chi-square difference tests were used to test whether Big Five personality dimensions were better predictors of problem behavior or the other around. These tests were only run for models in which cross-paths from personality to problem behavior, from problem behavior, or in both directions reached significance. Since 2 of the 10 models we ran contained no significant cross-paths in either direction, we just did 8 comparisons with chi-square difference tests. Only one test turned out to be significant, and another one almost reached significance. These tests indicated that Depression was a better predictor of Agreeableness then the other way around $(p<.05)$. Our results suggested that Aggression was a better predictor of Emotional Stability, but chi-square tests were just not significant ( $p$ $=.06)$.

Thus, problem behavior and personality did not predict one another in 2 out of 10 crosslagged panel models, and had equal predictive properties in 7 out of 8 of these models. In only 1 out of the 8 models problem behavior was a better predictor of personality than the other way around, and in none of the models personality was a better predictor of problem behavior than the other way around.

\subsection{Discussion}

The purpose of the present study was to examine longitudinal associations between the Big Five personality dimensions and problem behavior (i.e., Depression and Aggression) symptoms during adolescence, using cross-lagged panel models. Based on studies that used different models, we formulated the tentative hypothesis that personality could predict problem behavior (Asendorpf \& Denissen, 2006; Huey Jr. \& Weisz, 1997; Van Leeuwen et al., 2004), but also that problem behavior could predict personality over time (Ge \& Conger, 1999).

Several Big Five personality traits were found to predict problem behavior. In line with previous studies (Huey Jr. \& Weisz, 1997; Van Leeuwen et al., 2004), levels of Extraversion and Emotional Stability negatively predicted internalizing problem behavior (i.e., Depression). In addition, Conscientiousness was found to negatively predict internalizing problem behavior as well. Externalizing problem behavior (i.e., Aggression) was negatively predicted by Agreeableness and Conscientiousness. These findings are in line with those from previous studies (Huey Jr. \& Weisz, 1997; Van Leeuwen et al., 2004). Different from these 
previous studies, we also found that Openness was a negative predictor for externalizing problems. Overall, Big Five personality traits predicted both internalizing problems and externalizing problems.

Problem behavior was also found to predict Big Five personality traits. Extraversion, Agreeableness, Conscientiousness, and Emotional Stability were negatively predicted by internalizing problems. These findings were to a large extent comparable to results obtained by Ge and Conger (1999), who found that the MPQ superfactor of Positive Emotionality (which contains traits comparable to Extraversion and Agreeableness) was negatively predicted by psychological distress (i.e., internalizing problems), while the MPQ superfactor of Negative Emotionality (which can be perceived as being the opposite of Emotional Stability) was positively predicted by psychological distress. However, Ge and Conger (1999) found no associations of internalizing problems with the MPQ superfactor Constraint, which is comparable to the Big Five trait Conscientiousness.

Externalizing problems were found to negatively predict Agreeableness and Emotional Stability. The finding that Emotional Stability was negatively predicted by externalizing problems was comparable to Ge and Conger's (1999) findings, as they found that externalizing problems (i.e., delinquent behaviors) positively predicted Negative Emotionality.

Thus, our finding with regard to Big Five traits predicting problem behavior, and problem behavior predicting Big Five traits were to a large extent comparable to findings obtained in previous studies. As an important goal of the current study was to test whether our results favored the vulnerability hypothesis (i.e., personality as a predictor of problem behavior) or the scar hypothesis (i.e., problem behavior as a predictor personality) (e.g., Krueger \& Tackett, 2003; Tackett, 2006; Widiger et al., 1999), we tested whether personality was a better predictor of problem behavior or whether problem behavior was a better predictor of personality. Our results did not clearly favor the vulnerability or the scar hypothesis, as for $87,5 \%$ (i.e., 7 out of 8 ) of the models in which we found cross-paths in either direction, comparisons indicated that the predictive properties that personality and problem behavior exerted on one another were equivalent. Because we already noted that personality traits were significant predictors of problem behaviors, and problem behaviors were significant predictors of personality, both the vulnerability and the scar hypothesis were supported by our data. Hence, it is not only the presence of existing personality characteristics that increases the probability of the occurrence of specific problem behaviors, the presence of specific problem behaviors also complicates or 'scars' an individual's personality (Krueger \& Tackett, 2003). 
These findings underscore the importance of using a multi-wave cross-lagged panel models in future research, because these models allow to simultaneously test the vulnerability and the scar hypothesis, while correcting for previous associations between personality and problem behavior. As such, these models are more accurate in predicting the reciprocal associations between personality and problem behavior than ordinary regression analyses are.

Our study is not the first to suggest interplay between personality and problem behavior, instead of the unidirectional effects theorized in the vulnerability and the scar hypothesis. The corresponsive principle (e.g., Roberts, Caspi, \& Moffitt, 2003; Roberts \& Caspi, 2003) explains personality development from two mutually supportive processes: 'social selection' in which an individual selects an environment that corresponds to his or her personality traits, and 'social influence' in which experiences in the environment of one's choice further shape one's personality. In the current study, the corresponsive principle could be responsible for the reciprocal effect between Extraversion and Emotional Stability on the one hand, and internalizing problems on the other hand. More specifically, individuals low on Extraversion and Emotional Stability are described as being prone to developing internalizing problem behaviors, such as depression and anxiety (i.e., 'psychopathological selection'). In turn, this could lead to avoidance of public places, which leads to enhancing their problem behavior, consequently deepening their introverted and neurotic characteristics (i.e., 'psychopathological influence'). The reciprocal effects between Agreeableness and externalizing problems (i.e., Aggression) can be explained accordingly. Thus, less agreeable individuals could be prone to select more hostile social environments (i.e., deviant peer groups with high tolerance of Aggression), and the hostility experienced there could lead to a further decrease in their levels of Agreeableness. It should, however, be noted that this explanation of our finding is speculative. Therefore, future studies should aim to provide more direct tests of the corresponsive principle.

Overall, we found no differences between reciprocal influences of personality in problem behavior between boys and girls, or early to middle and middle to late adolescents. However, girls exhibited higher levels of relative stability in the depression models when compared to boys, whereas middle to late adolescent exhibited higher levels of relative stability in the aggression models when compared to early to middle adolescents. Even though differences in relative stability are generally thought to affect the magnitude of cross-lagged associations (e.g., Cole et al., 2006), the gender and age cohort differences in stability paths we found do not seem to have a significant effect on cross-lagged associations. 
We also found differences in the relative stability of personality and problem behavior. Boys' depressive symptoms were less stable than Extraversion, Conscientiousness, and Openness, but equally stable as Agreeableness and Emotional Stability. In girls, depressive symptoms were equally stable as Extraversion, Conscientiousness, Emotional Stability, and Openness, and even more stable then Agreeableness. One of the main differences between basic tendencies or core personality traits such as the Big Five, and characteristic adaptations or surface traits such as problem behavior symptoms, should theoretically be there relative stability (Asendorpf \& van Aken, 2003; McCrae \& Costa, 1999), with personality traits being more stable then problem behavior symptoms. As such, our results suggest that depressive symptoms seem to be more dispositional or closer to the core of personality in girls than in boys. With regard to aggression, another interesting pattern emerges. In early to middle adolescence, aggression and personality traits are equally stable, but in middle to late adolescence almost all Big Five traits, except for Agreeableness, are more stable then aggression. Thus, a distinction between Big five traits as core traits or basic tendencies versus problem behavior symptoms as surface traits or characteristic adaptations only emerges in middle to late adolescence, at least with regard to differences in relative stability. Overall, our findings suggest that one should consider gender and age as variables that can affect the distinction between personality traits and problem behavior symptoms.

\section{Strengths and Limitations of the Present Study}

An important strength of this study is that we were able to simultaneously test the vulnerability hypothesis (by testing the effects of the Big Five personality dimensions on problem behavior), and the scar hypothesis (by testing the effects of problem behavior on the Big Five personality dimensions; Krueger \& Tackett, 2003; Tackett, 2006; Widiger et al., 1999), using cross-lagged panel models. A second strength is that we tested these hypotheses in a large sample of adolescents drawn from the general population. Furthermore, because we had five annual waves of longitudinal data available on a cohort of early to middle adolescents and a cohort of middle to late adolescents, we were able to show that the reciprocal effects between personality dimensions and problem behavior remained similar throughout adolescence. Finally, we explicitly assessed whether associations between the Big Five personality dimensions and problem behavior were different or similar for boys and girls separately, instead of previous studies that just controlled for gender differences (Huey Jr. \& Weisz, 1997; Van Leeuwen et al., 2004). 
However, several limitations of the present study should also be addressed. The first limitation is that our findings on personality and problem behaviors were solely based on adolescent self-reports, which could result in biased answers and in shared method variance. Obviously, collecting data from multiple informants would improve our understanding of the associations between personality and problem behavior. However, since personality and internalizing behaviors might be more difficult to observe by others (e.g., Achenbach, McConaughy, \& Howell, 1987; Muris, et al., 2007), we were specifically interested in the feelings and opinions of adolescents themselves.

A second limitation is that we used questionnaires on personality and problem behaviors which could account for a certain amount of (item-) content overlap in both concepts. However, Prinzie, Onghena, and Hellinckx (2005) found that the associations between initial levels and changes in personality traits and externalizing problems of children did not change when overlapping items of personality and problem behavior scales were removed. Thus, longitudinal associations between personality and problem behavior are at least not to content overlap with regard to the items included in the measures. Still, individual differences in socially desirable responding or extremity of responding could inflate concurrent correlations between personality and problem behaviors. By using cross-lagged path analyses in which we controlled for initial associations between personality and problem behavior, we probably reduced the shared method variance at least to some extent (e.g., Neyer \& Asendorpf, 2001).

Third, cross-paths between personality and problem behavior and those in the opposite direction might seem rather weak at first sight. Previous studies (Asendorpf \& Denissen, 2006; Ge \& Conger, 1999; Huey Jr. \& Weisz, 1997; Van Leeuwen et al., 2004) generally found stronger associations. However, it should be noted that these studies used statistical analyses that are much less strict than the cross-lagged panel models that were used in the current study. In the estimation of cross-lagged paths in cross-lagged panel models, associations between initial levels (i.e., T1 associations), changes of personality and problem behavior (i.e., correlated change), and stability paths of personality and problem behavior are controlled for. Thus, there is less variance left to be explained by cross-paths (Cui, Donnellan, \& Conger, 2007). Hence, cross-paths are likely to be much weaker than in studies in which these strict cross-lagged panel models were not employed. Even studies relating highly similar constructs to one another, such as depression and self-esteem (Orth, Robins, \& Roberts, 2008), found standardized coefficients for cross-paths between .04 and .20.

Possibly, potential reciprocal effects between problem behavior and personality may have been small because of a fourth limitation, directly related to our use of cross-lagged 
panel models. In cross-lagged panel models, one controls for stability paths of included variables (i.e., personality and problem behavior). However, personality traits or problem behavior symptoms may turn out to be so stable that little variance remains left to be explained by other variables. To overcome this problem, Cole et al. (2006) applied Trait-State models to depression data in which a perfectly stable component (i.e., trait) and the part of the construct that does change (i.e., state) are distinguished. With this approach, Cole et al. (2006) showed that the effects of negative events on depression became much stronger once the trait component was statistically controlled for. It would have been valuable to apply Trait-State models to our data, but these models are very demanding as they require ideally 8 waves of data on at least 500 participants assuming at least moderate occasion factor stability (Cole, Martin, \& Steiger, 2005). The dataset employed in the current study does not match these demands.

Fifth, only subclinical levels of problem behaviors were assessed. Although the data reported here can be used as a baseline for clinical populations, they do not meet clinical criteria and the results of this study should not be equated with those from studies of adolescents with psychiatric disorders (e.g., Gjerde, Block, \& Block, 1988; Kim \& Smith, 1998).

A sixth limitation concerns the evidence we obtained for the vulnerability model. The vulnerability model is not the only model that could explain why personality predicts problem behavior. Tackett (2006) has argued that findings explained from a vulnerability perspective might as well be explained by the spectrum model. Thus, findings suggesting that personality traits predict problem behavior could also be perceived as evidence for specific kinds of problem behavior being more extreme manifestations of certain personality traits. For example, depression could be perceived as a very low level of Emotional Stability. Future work should focus on innovative study designs to disentangle vulnerability and spectrum model explanations for why personality predicts problem behavior.

Despite the mentioned limitations, the present study provides an important insight into the longitudinal associations between Big Five personality dimensions and problem behavior in early to middle and middle to late adolescent boys and girls. Our results clearly indicated that it is not only personality that affects problem behavior, but it is also problem behavior that affects personality. 


\section{Chapter 4}

A Developmental Typology of Adolescent Personality ${ }^{4}$

${ }^{4}$ Klimstra, T. A., Hale, W. W., Raaijmakers, Q. A. W., Branje, S. J. T., \& Meeus, W. H. J. (in press). A developmental typology of adolescent personality. European Journal of Personality. 


\begin{abstract}
The purpose of the current study is to examine whether Block's personality types (i.e., Resilients, Undercontrollers, and Overcontrollers) are replicable as developmental trajectories. We applied a Latent Class Growth Analysis (LCGA) framework to five-annualwave data on a sample of early to middle adolescents $(n=923)$. Our results showed that Block's Resilients, Undercontrollers, and Overcontrollers are indeed replicable as developmental trajectories across adolescence. These developmental types were related to problem behaviour in a similar way as types found in studies using cross-sectional data were. As such, Resilients reflected low levels of problem behavior, Undercontrollers had high levels of delinquency, and Overcontrollers had high levels of depression. Implications and suggestions for further research are discussed.
\end{abstract}




\subsection{Introduction}

The most commonly used person-centred approach to personality was introduced by Block and Block (1980). They proposed three personality types: Resilients, Undercontrollers, and Overcontrollers. These three types differ in their amount of ego-control and egoresiliency. Ego-control indicates the degree of impulse control, whereas ego-resiliency refers to the capacity of a person to modify levels of ego-control as a function of environmental demands. Resilients are characterized by high levels of ego-resiliency, and are, as a result, well-able to adjust their levels of ego-control to environmental demands. Both Overcontrollers and Undercontrollers reflect low levels of ego-resiliency, but whereas Overcontrollers are characterized by high levels of ego-control, Undercontrollers are characterized by low levels of ego-control. Personality types are by no means perfectly discrete entities, as boundaries between types may be somewhat fuzzy (Asendorpf, Borkenau, Ostendorf, \& van Aken, 2001). That is, relatively small differences on a certain trait can, for example, result in an individual being classified as a Resilient instead of as an Overcontroller. Nevertheless, the value of the types as a non-arbitrary multivariate classification has been proofed in a wide range of studies, involving children, adolescents, and adults (e.g., Asendorpf et al., 2001).

In a sample of adolescent boys, Robins, John, Caspi, Moffitt, and Stouthamer-Loeber (1996) demonstrated that Resilients, Overcontrollers, and Undercontrollers have specific Big Five personality profiles. Resilients had the best adjusted personality profile of the three types, reflected by the highest scores on four of the Big Five personality dimensions: Conscientiousness, Emotional Stability, Openness to Experience, and Extraversion. On the fifth dimension, Agreeableness, Resilients had above average scores, but Overcontrollers had the highest scores. Compared to the two other types, Overcontrollers had the lowest scores on Extraversion and Emotional Stability, and Undercontrollers had the lowest scores on Agreeableness and Conscientiousness. These findings have been replicated several times (for overviews, see for example: Hart, Atkins, \& Fegley, 2003; van Lieshout, 2000), and later studies demonstrated that adolescent personality types can be constructed directly from Big Five questionnaire data (e.g., Akse, Hale, Engels, Raaijmakers, \& Meeus, 2004; Asendorpf et al., 2001; Dubas, Gerris, Janssens, \& Vermulst, 2002). With regard to gender differences, boys usually are overrepresented among Undercontrollers (e.g., Akse et al., 2004; Asendorpf et al., 2001), while girls tend to be overrepresented among Resilients and/or Overcontrollers (e.g., Akse et al., 2004; Dubas et al, 2002). Several studies further demonstrated that Resilients, Undercontrollers, and Overcontrollers have distinctive patterns of problem 
behavior (e.g., Akse et al., 2004; Robins et al., 1996). Resilients usually display little problem behavior, Undercontrollers are described by high levels of externalizing problems (e.g., delinquency), and Overcontrollers by high levels of internalizing problems (e.g., depression). Denissen, Asendorpf, and van Aken (2008) showed that personality types are not only concurrently related to problem behavior, but demonstrated that these types can also longitudinally predict shyness and aggressiveness. In addition, the childhood personality types derived by Denissen et al. (2008) even longitudinally predicted the timing of demographic transitions, such as leaving the parental home, establishing a romantic relationship, and finding a job. Thus, personality types are powerful predictors of current and later adjustment, and demographic transitions.

In the aforementioned studies, the emphasis is on the classification of individuals into a certain personality type at a certain measurement occasion. It is also possible to examine personality types from a developmental perspective. One way to assess development within a typological framework is to examine stability and change in personality type membership across different time points. Previous studies that used this approach to examine long-term stability of type membership in childhood (Asendorpf \& van Aken, 1999), from childhood to adolescence (Asendorpf, 2003), and in adolescence (Akse, Hale, Engels, Raaijmakers, \& Meeus, 2007; van Aken \& Dubas, 2004) found that more than $40 \%$ of individuals changed from one type to another. This could possibly be attributed to the previously mentioned fuzzy boundaries between personality types. In addition, studying transitions from one static type to another static type across several measurement occasions can result in serious interpretation problems. For example, examining transitions between three types across three waves results in $3^{3}=27$ possible cross-time personality types (van Aken \& Dubas, 2004). Another problem is that individual developmental trajectories and normative development within personality types are lost in such an approach (e.g., Block, 1971), as transitions across waves between types that have been constructed using the same cluster means could actually be caused by normative developmental changes in the underlying personality traits.

Therefore, Block (1971) originally made a case for studying 'types of personality development, not types of personality' (p. 113). According to Block, a personality typology should reflect the way personality types manifest themselves during longer and substantial periods in the life course. In other words, personality types should incorporate normative developmental trends in personality. He stated that "we need to be able to plot the various separate trend-lines of our personality types, attending both to the cross-sectional comparisons 
available at each slice of time and to the directions and significance of the changes observed over the years" (p. 113).

Only three studies have taken a true developmental approach to the study of personality types. Block (1971) was the first to study a developmental typology of personality; his study focused on the period from adolescence into adulthood. Block's typology was, however, only based on small samples of men and women (84 and 86 participants, respectively). Nevertheless, it inspired Morizot and Le Blanc $(2003 ; 2005)$ three decades later to re-examine a developmental typology of personality from adolescence into adulthood (across ages 14, 16, 30, and 40) in a sample of men who had been in jail before the onset of the study (Morizot \& Le Blanc, 2003) and in a sample of men drawn from the general population (Morizot \& Le Blanc, 2005). In both studies four developmental types were found, but in both cases three of these four developmental personality types were comparable to the personality types that are typically found in cross-sectional studies (i.e., Resilients, Undercontrollers, and Overcontrollers). Thus, non-developmental personality types are at least to some extent replicable as developmental personality types.

In the current study, we will investigate a developmental personality typology for early to middle adolescent boys and girls (ages 12 to 16) using data from a large scale ( $N=$ 923) five-annual-wave longitudinal research project. We will thereby extend the studies of both Block (1971) and Morizot and LeBlanc (2003, 2005), who focused on small samples of men and covered the period from adolescence into adulthood with only a limited number of measurement occasions in adolescence. Furthermore, for the identification of longitudinal personality types, the earlier mentioned studies have used methods such as Q-factor analysis and longitudinal cluster analysis, which do not take measurement error into account. The present study uses Latent Class Growth Analysis (LCGA; Nagin, 1999, 2005), a relatively new technique that allows to identify several homogeneous groups within a heterogeneous sample, based on the initial levels (referred to as intercepts) and growth rate (referred to as slopes) of individuals on a certain set of variables (i.e., Big Five personality characteristics), while controlling for measurement error. It should be noted that LCGA and similar techniques do not create perfectly discrete types (Johnson, Hicks, McGue, \& Iacono, 2007). Boundaries between types are at best fuzzy, just like the boundaries between types derived in crosssectional studies are (Asendorpf et al., 2001).

In the current study, we expect to find at least three developmental personality types, resembling the three 'static' personality types consistently found in previous studies: Resilients, Undercontrollers and Overcontrollers. In addition, we will examine how our 
longitudinal trajectories of personality are associated with internalizing (i.e., depression) and externalizing (i.e., delinquency) problem behavior. Finally, gender differences in the prevalence of developmental personality types will be explored.

\subsection{Method}

\section{Participants}

Data for this study were collected as part of an ongoing longitudinal research project on CONflict And Management Of RElationships (CONAMORE; Meeus et al., 2006). Data of the first five annual measurement waves were used. The longitudinal sample consisted of 923 early to middle adolescents ( 468 boys and 455 girls; $M_{\text {age }}=12.4$ years $(S D=.59)$ during the first wave of measurement), who attended various randomly selected high schools in the province of Utrecht, The Netherlands. With regard to ethnicity, 83.4\% identified themselves as Dutch and $16.6 \%$ indicated that they belonged to ethnic minorities (e.g., Surinamese, Antillean, Moroccan, Turkish). As these figures were $79 \%$ and $21 \%$, respectively, in the general Dutch adolescent population (Statistic Netherlands, 2008), Dutch youth was slightly overrepresented. Because participants were distributed across all available levels in the Dutch educational system, and Socio-Economic Status ranged from high to low, our sample is quite representative for the general Dutch population of early to middle adolescents.

Sample attrition was $1.2 \%$ across waves. Missing item values were estimated in SPSS, using the EM-procedure. Across waves 5.2\% of the data was missing. Little's Missing Completely At Random Test (Little, 1988) revealed a normed $\chi^{2}\left(\chi^{2} / \mathrm{df}\right)$ of 1.31 which, according to guidelines by Bollen (1989), indicates a good fit between sample scores with and without imputation.

\section{Procedure}

Participants and their parents received an invitation letter, describing the research project and goals, and explaining the possibility to decline from participation. More than $99 \%$ of the approached high school students decided to participate. All participants signed the informed consent form.

\section{Measures}

Personality. Personality was self-rated by participating adolescents, with the shortened Dutch version of Goldberg's Big Five questionnaire (Gerris et al., 1998; Goldberg, 1992). This 
Likert-type measure, with a response format ranging from 1 (completely untrue) to 7 (completely true), was used to assess the Big Five personality dimensions. Each Big Five dimension was indicated by 6 items, such as: talkative (Extraversion), sympathetic (Agreeableness), systematic (Conscientiousness), worried (Emotional Stability, reversed scored), and creative (Openness to Experience). Reliability was acceptable at all included measurement waves, as Cronbach's alphas across all Big Five Scales ranged from .76 to .88. Depression. Depressive symptoms were measured with the Children's Depression Inventory (CDI; Kovacs, 1985), a self-report questionnaire aimed at screening (subclinical) depressive symptomatology in children and adolescents. The CDI consists of 27 items (e.g., "I'm sad all the time"). The items were scored on a 3-point scale, ranging from 1 (false), to 3 (very true). Reliability of the CDI was high at all included measurement waves, with Cronbach's alphas ranging from .89 to .94 .

Delinquency. To measure delinquency, a self-report questionnaire measuring the frequency of several minor offences (Baerveldt, van Rossem, \& Vermande, 2003) was used. The use of self-report data is widespread in criminology, and the instrument is valid when restricted to petty crime (Baerveldt, 2000). Adolescents were asked how many times they had committed 16 minor offences, such as stealing a bike and deliberately damaging or breaking something in the street, in the past 12 months. The items were scored on a 4-point scale, ranging from 0 (never) to 3 (four times or more). Reliability was high at all included measurement waves, as Cronbach's alphas ranged from .83 to .93 .

\subsection{Results}

We used Latent Class Growth Analysis (LCGA; Nagin, 1999, 2005) to investigate a developmental typology of adolescent personality. In LCGA, homogeneous subgroups characterized by more or less the same level and change rate on a set of variables are derived from a heterogeneous sample. To determine whether there were such potential subgroups within our sample, we first assessed univariate Latent Growth Models (LGM; e.g., Duncan, Duncan, Stryker, Li, \& Alpert, 1999), using Mplus (Muthén \& Muthén, 2007).

\section{Univariate Latent Growth Models}

We specified univariate LGMs (growth factor loadings $0,1,2,3$, and 4, for the five consecutive annual measurement waves, respectively) for all Big Five factors, to determine what kind of growth characterized our data best, and whether there was significant variance in 
growth (i.e., significant slope variance). The best models have the lowest chi-squares corrected for degrees of freedom (Satorra \& Bentler, 2001) and Root Mean Square Errors of Approximation (RMSEA), and the highest Comparative Fit Indices (CFI) and Tucker Lewis Indices (TLI) (Kline, 2005).

For all Big Five factors, the best fitting models included linear and quadratic slopes. Furthermore, intercepts and slopes within models were allowed to correlate, as this significantly improved model fit. Descriptive statistics are displayed in Table 4.1, fit indices of the univariate LGMs appear in Table 4.2.

Table 4.1

Descriptive Statistics of Big Five Personality Traits and Problem Behavior

\begin{tabular}{|c|c|c|c|c|c|}
\hline & $\mathrm{T} 1$ & $\mathrm{~T} 2$ & $\mathrm{~T} 3$ & $\mathrm{~T} 4$ & T5 \\
\hline & $M(S D)$ & $M(S D)$ & $M(S D)$ & $M(S D)$ & $M(S D)$ \\
\hline \multicolumn{6}{|l|}{ Personality } \\
\hline Extraversion & $4.91(1.01)$ & $4.84(1.06)$ & $4.90(1.08)$ & $4.85(1.12)$ & $4.94(1.11)$ \\
\hline Agreeableness & $5.06(1.08)$ & $5.20(.99)$ & $5.16(.93)$ & $5.30(.87)$ & $5.44(.74)$ \\
\hline Conscientiousness & $4.13(1.11)$ & $4.22(1.16)$ & $4.09(1.16)$ & $4.08(1.18)$ & $4.17(1.20)$ \\
\hline Emotional Stability & $4.64(1.12)$ & $4.48(1.11)$ & $4.59(1.07)$ & $4.60(1.06)$ & $4.63(1.07)$ \\
\hline Openness & $4.39(1.08)$ & $4.58(1.07)$ & $4.48(1.04)$ & $4.60(1.01)$ & $4.70(.99)$ \\
\hline \multicolumn{6}{|l|}{ Problem Behavior } \\
\hline Depression & $1.16(.26)$ & $1.18(.23)$ & $1.18(.23)$ & $1.19(.24)$ & $1.18(.21)$ \\
\hline Delinquency & $1.18(.38)$ & $1.13(.28)$ & $1.15(.30)$ & $1.15(.28)$ & $1.17(.30)$ \\
\hline
\end{tabular}

Table 4.2

Fit Statistics of Univariate LGMs

\begin{tabular}{lcccccc}
\hline & \multicolumn{1}{c}{$\chi^{2}$} & $d f$ & CFI & TLI & RMSEA & $\begin{array}{c}\text { 90\% C.I. of } \\
\text { RMSEA }\end{array}$ \\
\hline Extraversion & $15.90^{*}$ & 6 & 1.00 & .99 & .04 & $.02, .07$ \\
Agreeableness & $22.15 * *$ & 6 & .99 & .98 & .05 & $.03, .08$ \\
Conscientiousness & $22.02 * *$ & 6 & .99 & .99 & .05 & $.03, .08$ \\
Emotional Stability & $23.43 * * *$ & 6 & .99 & .98 & .06 & $.03, .08$ \\
Openness & $27.62 * * *$ & 6 & .99 & .98 & .06 & $.04, .09$ \\
\hline
\end{tabular}

Note. $* p<.05, * * p<.01, * * * p<.001$.

As we were mainly interested in identifying different developmental trajectories of adolescent, we merely focused on variance around mean intercepts, and mean linear and/or quadratic slope factors. For a discussion of mean-level changes for our sample as a whole, the 
reader is referred to Klimstra, Hale, Raaijmakers, Branje and Meeus (2009). Because we found significant variance around mean intercepts and slope factors (see Table 4.3), we could proceed to the next step: identifying subgroups that displayed distinct developmental personality profiles with regard to initial levels (i.e., intercepts) and rates of change (i.e., slopes) for the Big Five personality factors with LCGA.

Table 4.3

Intercepts, and Growth Parameters for Univariate Latent Growth Curve Models

\begin{tabular}{lcccccc}
\hline & \multicolumn{2}{c}{ Intercept } & \multicolumn{2}{c}{ Linear Slope } & \multicolumn{2}{c}{ Quadratic Slope } \\
\hline & $M$ & $\sigma^{2}$ & $M$ & $\sigma^{2}$ & $M$ & $\sigma^{2}$ \\
\hline Extraversion & $4.90 * * *$ & $.50 * * *$ & -.05 & $.20 * * *$ & $.01 *$ & $.01 * * *$ \\
Agreeableness & $5.10^{* * *}$ & $.49 * * *$ & .02 & $.26 * * *$ & $.02 *$ & $.01 * * *$ \\
Conscientiousness & $4.17 * * *$ & $.67 * * *$ & $-.06 *$ & $.19 * * *$ & $.01 *$ & $.01 *$ \\
Emotional Stability & $4.61 * * *$ & $.71 * * *$ & -.05 & $.37 * * *$ & $.02 *$ & $.02 * * *$ \\
Openness & $4.43 * * *$ & $.58 * * *$ & .04 & $.24 * * *$ & .01 & $.01 * * *$ \\
\hline Note. $M=$ mean; $\sigma^{2}=$ variance around the mean. $* p<.05, * * p<.01, * * * p<.001$. &
\end{tabular}

\section{Latent Class Growth Analysis}

LCGA is aimed at finding the smallest number of classes capturing most variance among individuals with regard to initial levels and change on variables included in the model. Because univariate LGMs indicated that personality development had a curvilinear shape, we specified linear as well as quadratic slopes in the LCGAs. In LCGAs, variances of the estimated means of intercepts and linear as well as quadratic growth terms are constrained to zero. When compared to related procedures in which variances of intercepts and slopes are freely estimated (e.g., Growth Mixture Modeling; Muthén \& Muthén, 2000), LCGA results in a somewhat less optimal model fit, but produces more clearly distinguishable classes (Nagin, 2005).

We used several criteria, outlined by Muthén and Muthén (2000), to determine the number of latent classes (i.e., developmental personality types) that best characterized our data. First, we used the Sample Size Adjusted Bayesian Information Criterion (SSA-BIC; e.g., Schwarz, 1978) and the Lo-Mendell-Rubin Likelihood Ratio Test (LMR-LRT; Lo, Mendell, $\&$ Rubin, 2001). The optimal model has the lowest SSA-BIC, while a significant LMR-LRT indicates that a model with $k$ classes is better than a model with $k-1$ classes. Second, we assessed the index of classification accuracy: the entropy. The entropy can range from .00 to 1.00, with higher figures representing a more accurate classification (Hix-Small, Duncan, 
Duncan, \& Okut, 2004). Third, theoretical meaningfulness of classes in the various solutions was considered. If a class found in a solution with $k$ classes was found to be a slight variation of a class already found in a solution with $k-1$ classes, the most parsimonious solution was chosen (Muthén \& Muthén, 2000).

With this approach, a three-class solution proved to be better than a two-class solution. Adding a fourth class did improve model fit, but adding a fifth class did not result in an improvement of model fit (see Table 4.4). In addition, the fourth class in the four-class solution proved to be a slight variation of one of the classes that was already present in the three-class solution. More specifically, this fourth class represented Resilients with lower scores on Conscientiousness, and slightly lower scores on Agreeableness. Because of this, we chose the three-class solution as our final solution.

Table 4.4

Fit Indices of Various LCGA Solutions

\begin{tabular}{lccc}
\hline & SSA-BIC & Entropy & LMR-LRT \\
\hline One class & 67964.01 & - & - \\
Two classes & 65975.05 & .82 & $2028.82^{*}$ \\
Three classes & 63939.63 & .87 & $1853.30^{* *}$ \\
Four classes & 63119.46 & .88 & $870.63^{* *}$ \\
Five classes & 62574.09 & .88 & 598.32 \\
\hline
\end{tabular}

Note. ${ }^{*}=p<.05, * *=p<.01$. An insignificant LMR-LRT indicates that a solution with $k$ classes is not significantly better than a solution with $k$ - 1 classes. In a one-class solution, it is impossible to calculate the Entropy and run an LMR-LRT test.

In line with our expectations, the three classes we found could be described as Resilients (33.9\% of the sample, $n=313)$, Undercontrollers ( $24.7 \%$ of the sample, $n=228)$, and Overcontrollers $(41.4 \%$ of the sample, $n=382)$. Means and variances of growth parameters for the three types are displayed in Table 4.5, and are plotted in Figure 4.1.

Confidence intervals revealed that Resilients had the highest intercepts on four of the Big Five dimensions. For Emotional Stability, the intercepts of Undercontrollers were somewhat higher than those of Resilients, but these differences did not reach significance. Overcontrollers had equally high intercepts as Resilients for Openness and Conscientiousness. Undercontrollers had intermediate intercepts on Extraversion, and the lowest intercepts for Agreeableness, Conscientiousness, and Openness. Overcontrollers had intermediate intercepts for Agreeableness and the lowest intercepts for Emotional Stability and Extraversion.

As personality development in Resilients, Undercontrollers, and Overcontrollers was characterized by curvilinear growth, which is a function of both linear and quadratic slope 
parameters (displayed in Table 4.5), it is hard to judge differences in growth from a table.

Therefore, growth of the personality types for each Big Five trait is displayed in Figure 4.1.

Figure 4.1 shows that levels of Extraversion increased for Resilients, were stable for Undercontrollers, and decreased in Overcontrollers. Levels of Agreeableness increased for all three types. With regard to Conscientiousness, change rates did not reach significance. Levels of Emotional Stability did not change significantly for Resilients and Undercontrollers.

Overcontrollers displayed a curvilinear pattern of change, in which a decrease in Emotional Stability was followed by an increase. Resilients and Undercontrollers became more open to experience as they grew older, whereas Overcontrollers' levels of Openness were stable across the period covered in the current study.

Table 4.5

Mean Intercepts and Growth Factors

$\begin{array}{ccc}\text { Resilients } & \text { Undercontrollers } & \text { Overcontrollers } \\ M(95 \% \text { C.I. }) & M(95 \% \text { C.I. }) & M(95 \% \text { C.I. })\end{array}$

Intercepts

$\begin{array}{lll}\text { Ex } & 5.43 * * * a \mathrm{a}(5.33,5.54) & 5.07 * * * \mathrm{~b}(4.88,5.26) \\ \mathrm{Ag} & 5.51 * * * \mathrm{a}(5.38,5.64) & 4.41^{* * * \mathrm{~b}}(4.10,4.72) \\ \mathrm{Co} & 4.35 * * * \mathrm{a}(4.15,4.56) & 3.63 * * * \mathrm{~b}(3.42,3.85) \\ \text { ES } & 4.88^{* * * \mathrm{a}}(4.74,5.02) & 5.03 * * * \mathrm{a}(4.82,5.25) \\ \text { Op } & 4.80 * * * \mathrm{a}(4.63,4.96) & 3.63 * * * \mathrm{~b}(3.35,3.90)\end{array}$

$4.34 * * * \mathrm{c}(4.23,4.46)$

Linear Slopes

$\begin{array}{lllllll}\text { Ex } & .16^{* * \mathrm{a}}(.06, .26) & .00^{\mathrm{a}} & (-.13, .14) & -.24 * * * \mathrm{~b} & (-.34,-.15) \\ \mathrm{Ag} & .17^{* * * \mathrm{a}}(.09, .25) & -.03^{\mathrm{a}, \mathrm{b}}(-.23, .16) & -.06^{\mathrm{b}} & (-.15, .03) \\ \mathrm{Co} & -.04^{\mathrm{a}} & (-.14, .06) & -.08^{\mathrm{a}} & (-.23, .07) & -.02^{\mathrm{a}} & (-.11, .07) \\ \mathrm{ES} & .07^{\mathrm{a}} & (-.06, .19) & -.02^{\mathrm{b}} & (-.21, .16) & -.18^{* *} *^{\mathrm{b}} & (-.29,-.07) \\ \mathrm{Op} & .22^{* * * \mathrm{a}}(.13, .31) & -.12^{\mathrm{b}} & (-.30, .06) & .01^{\mathrm{b}} & (-.08, .10)\end{array}$

Quadratic Slopes

$\begin{array}{lll}\text { Ex } & -.02 * \text { a } & (-.05, .00) \\ \text { Ag } & -.02 * *^{\mathrm{a}} & (-.04,-.01) \\ \mathrm{Co} & .00^{\mathrm{a}} & (-.14, .06) \\ \mathrm{ES} & -.01^{\mathrm{a}} & (-.06, .19) \\ \mathrm{Op} & -.03 * *^{\mathrm{a}} & (-.05,-.01)\end{array}$

$.01^{\mathrm{a}, \mathrm{b}}(-.02, .04)$

$.05^{* * * \mathrm{~b}}(.03, .07)$

$.05 * \mathrm{~b}(.00, .09)$

$.03 * * \mathrm{~b} \quad(.01, .05)$

$.03^{\mathrm{a}}(.00, .06)$

$.00^{\mathrm{a}} \quad(-.02, .02)$

$.01^{\mathrm{a}, \mathrm{b}}(-.03, .05)$

$.04 * * * \mathrm{~b}(.02, .07)$

$.05 * \mathrm{~b}(.01, .09)$

$.01^{\mathrm{a}, \mathrm{b}} \quad(-.01, .02)$

Note. $\mathrm{Ex}=$ Extraversion; $\mathrm{Ag}=$ Agreeableness; Co = Conscientiousness; $\mathrm{ES}=$ Emotional Stability; Op = Openness. Within a row, different superscripts indicate significant differences $(p<.05)$ between Resilients, Undercontrollers, and Overcontrollers in Extraversion, Agreeableness, Conscientiousness, Emotional Stability and Openness. Significance of differences between personality types on Big Five dimensions were obtained by comparing $95 \%$ confidence intervals of intercept and slope factors. These $95 \%$ confidence intervals are displayed between brackets. Effect sizes (Cohen's $d$ 's) of significant between-group differences in intercepts and slope factors range from .43 to 2.17 . 
Developmental Typology
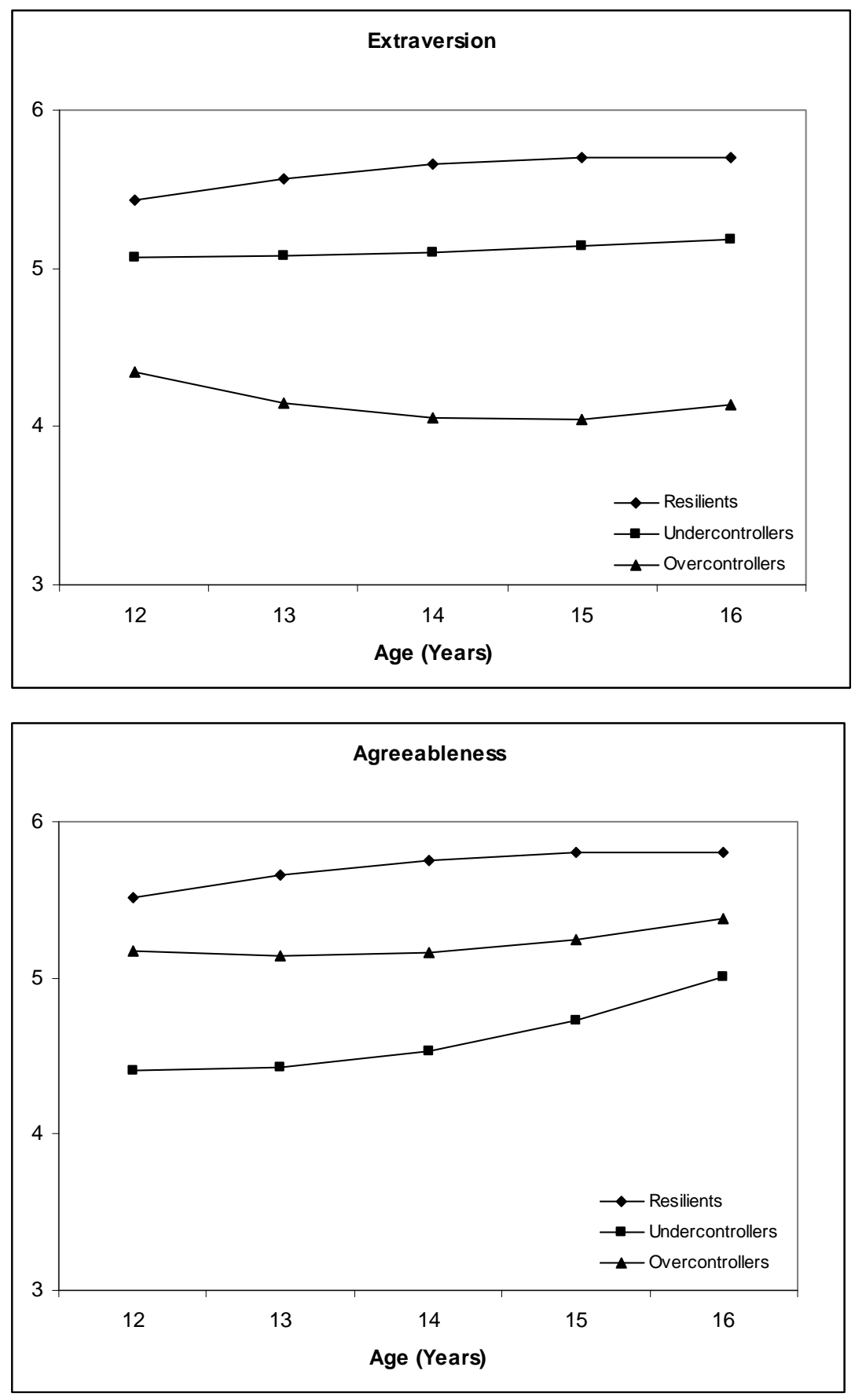
Chapter 4
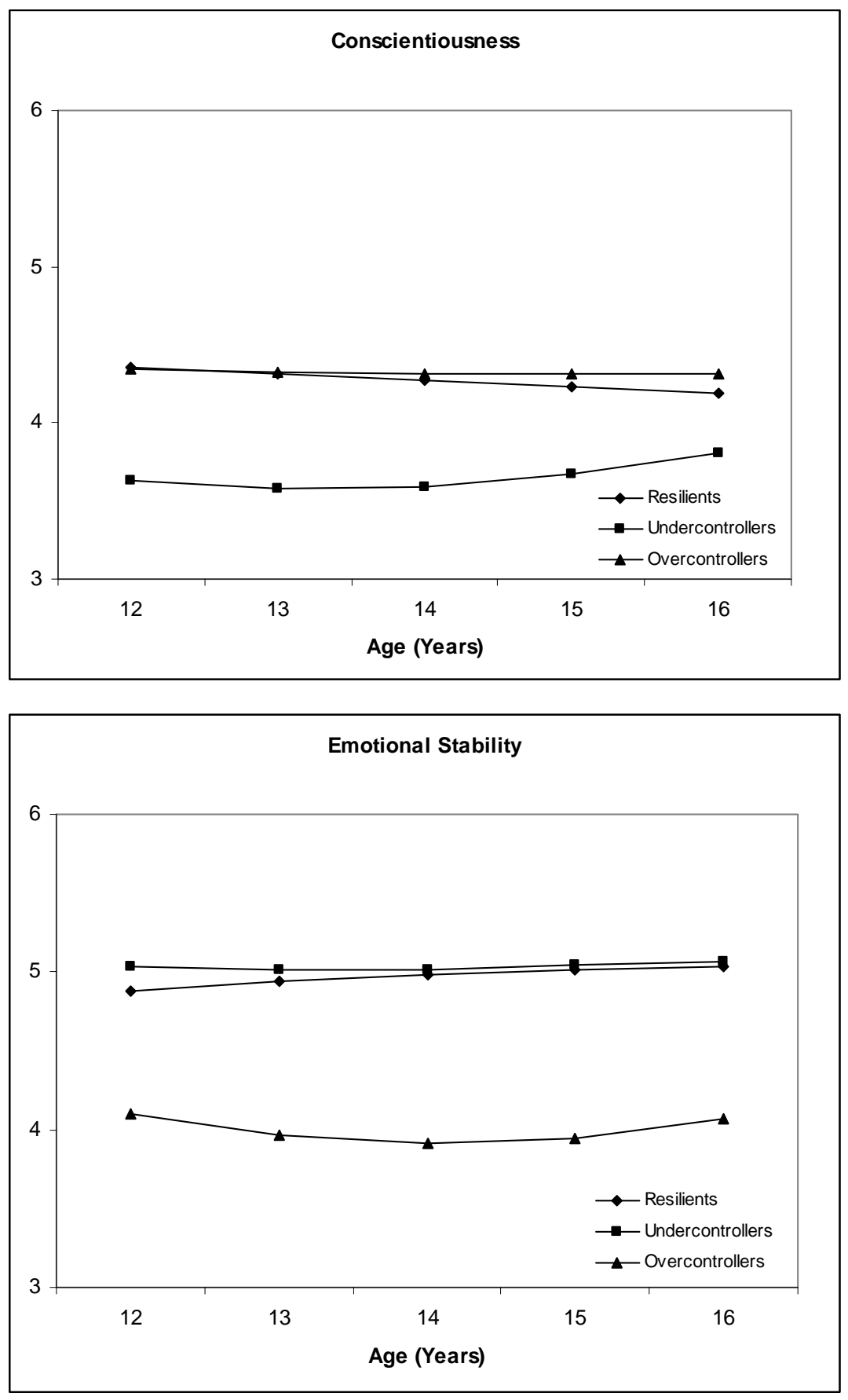


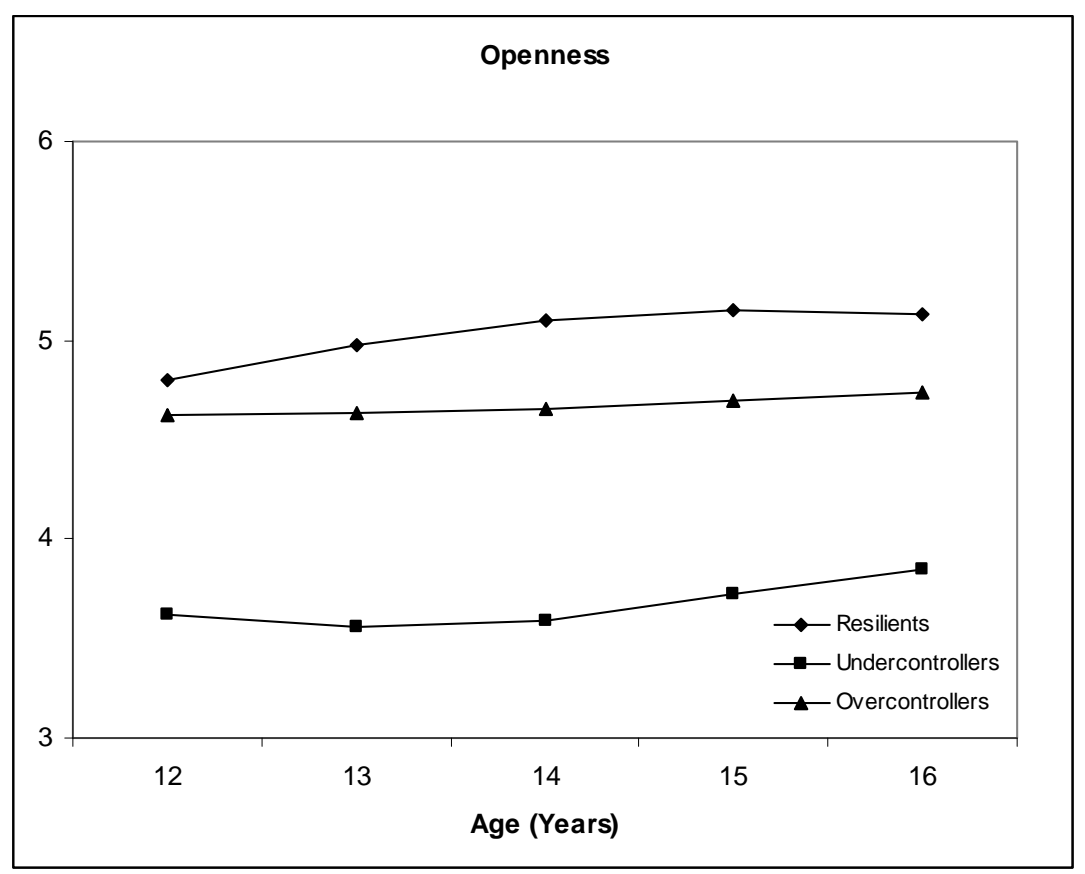

Figure 4.1a, b, $c, d$, e. Estimated Growth of Personality in Resilients, Undercontrollers, and Overcontrollers for Extraversion, Agreeableness, Conscientiousness, Emotional Stability, and Openness, respectively.

Substantial gender differences in the prevalence of the three personality types were found $\left(\chi^{2}(2)=42.04 ; p<.001\right)$. Boys were relatively more often classified as Undercontrollers than girls (33.8\% and $15.4 \%$, respectively; $\left.\chi^{2}(1)=41.88 ; p<.001\right)$, but less often classified as Resilients $\left(29.3 \%\right.$ and $38.7 \%$, respectively; $\left.\chi^{2}(1)=9.11 ; p=.003\right)$ and Overcontrollers $\left(37.0 \%\right.$ and $45.9 \%$, respectively; $\left.\chi^{2}(1)=7.65 ; p=.006\right)$.

\section{Developmental Personality Types and Problem Behaviour}

To further validate our developmental typology, we assessed whether Resilients, Undercontrollers, and Overcontrollers were described by distinct initial levels (i.e., intercepts) and change rates (i.e., slopes) of problem behaviour (i.e., depression and delinquency). For this purpose, we used multigroup LGMs, with the personality types as groups. Descriptive statistics of depression and delinquency are provided in Table 4.1.

Development of both depression and delinquency was best described by curvilinear growth. Fits of the models for both depression $\left(\chi^{2}(21)=20.24\right.$ (n.s.); CFI $=1.00$; TLI $=1.00$; RMSEA $=.00(90 \%$ C.I. $=.00, .05))$ and delinquency $\left(\chi^{2}(21)=49.31(p<.001) ;\right.$ CFI $=.98$; $\mathrm{TLI}=.97 ; \mathrm{RMSEA}=.07(90 \%$ C.I. $=.04, .09))$ were good. Initial levels and growth of depression and delinquency are shown in Table 4.6 and displayed in Figure 4.2. 
Confidence intervals revealed that Resilients had the lowest initial levels of both depression and delinquency. Overcontrollers had the highest initial levels of depression, and Undercontrollers were characterized by the highest levels of delinquency. Undercontrollers displayed a curvilinear change pattern for depression, which resulted in little change across time. Resilients and Overcontrollers did not change significantly with regard to depression. For delinquency, Overcontrollers displayed a significant curvilinear growth pattern that resulted in very little change over time. Resilients and Undercontrollers did not exhibit significant changes in delinquency.

Table 4.6

Mean Intercepts and Growth Factors of Problem Behavior

\begin{tabular}{|c|c|c|c|}
\hline & Resilients & Undercontrollers & Overcontrollers \\
\hline \multicolumn{4}{|l|}{ Intercepts } \\
\hline Depression & $1.11 * * * \mathrm{a}(1.09,1.13)$ & $1.14 * * * \mathrm{a}(1.11,1.17)$ & $1.22 * * * \mathrm{~b}(1.19,1.24)$ \\
\hline Delinquency & $1.12 * * * \mathrm{a}(1.09,1.14)$ & $1.27 * * * \mathrm{~b}(1.20,1.33)$ & $1.14 * * * \mathrm{a}(1.11,1.17)$ \\
\hline \multicolumn{4}{|l|}{ Linear Slopes } \\
\hline Depression & $.01^{\mathrm{a}}(-.01, .03)$ & $.03 *^{\mathrm{a}}(.00, .06)$ & $.03^{\mathrm{a}}(.00, .05)$ \\
\hline Delinquency & $.00^{\mathrm{a}}(-.02, .03)$ & $-.05^{\mathrm{a}}(-.10, .00)$ & $-.03 *^{\mathrm{a}}(-.06,-.01)$ \\
\hline \multicolumn{4}{|l|}{ Quadratic Slopes } \\
\hline Depression & $.00^{\mathrm{a}}(.00, .00)$ & $-.01 * * \mathrm{a}(-.02, .00)$ & $.00^{\mathrm{a}}(-.01, .00)$ \\
\hline Delinquency & $.00^{\mathrm{a}}(.00, .01)$ & $.01^{\mathrm{a}} \quad(.00, .02)$ & $.01 * * \mathrm{a}(.00, .01)$ \\
\hline
\end{tabular}

Note. $*=p<.05, * *=p<.01, * * *=p<.001$. Within a row, different superscripts indicate significant differences $(p<.05)$ between Resilients, Undercontrollers, and Overcontrollers in depression and delinquency. Significance of differences between personality types on Big Five dimensions were obtained by comparing $95 \%$ confidence intervals of intercept and slope factors. These $95 \%$ confidence intervals are displayed between brackets. Effect sizes (Cohen's $d$ 's) of significant between-group differences in intercepts and slope factors range from .59 to .61 . 
Developmental Typology
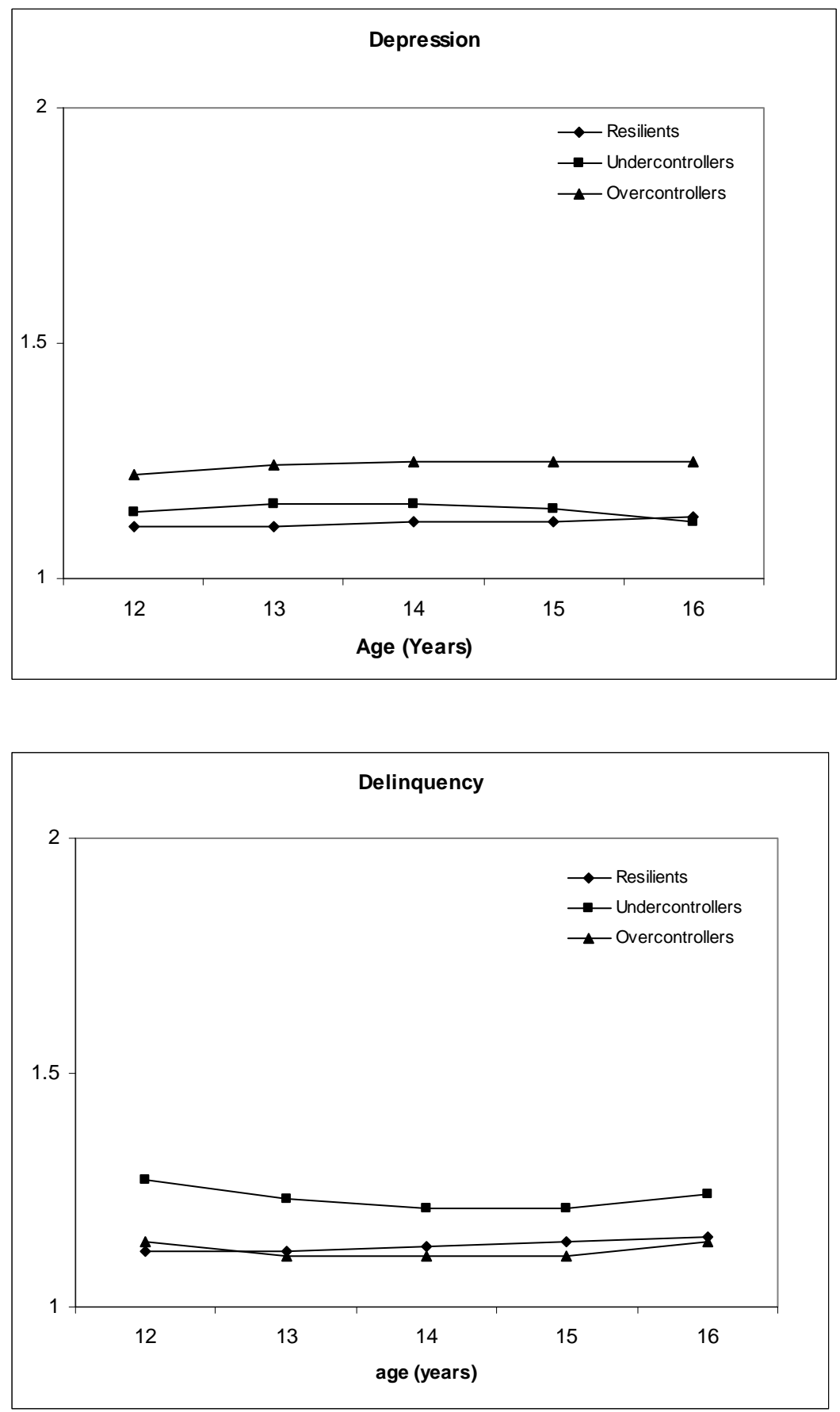

Figure 4.2a, $b$. Estimated Growth of Depression and Delinquency in Resilients, Undercontrollers, and Overcontrollers.

\subsection{Discussion}

The purpose of this study was to examine a developmental typology of adolescent personality. The current study was the first with an exclusive focus on a developmental personality typology of adolescence, applying the recently developed LCGA framework (LCGA; Nagin, 1999, 2005). 
We decided to label the three classes we found with LCGA as Resilients, Undercontrollers, and Overcontrollers, because their Big Five profiles were comparable to the profiles typically found in previous studies that applied cross-sectional clustering techniques to identify these personality types (e.g., Akse et al., 2007; Dubas et al., 2002; Robins et al., 1996). Like in these previous studies, Resilients had the most favourable personality profile with high scores on all Big Five traits, Undercontrollers scored low on Agreeableness, Conscientiousness, and Openness, and Overcontrollers displayed low levels of Extraversion and Emotional Stability. In addition, the longitudinal personality types we found in the current study were related to problem behavior in a similar way as personality types found in the just mentioned cross-sectional studies. Thus, Resilients had low levels of problem behavior, Undercontrollers were characterized by high levels of externalizing behavior (i.e., delinquency), and Overcontrollers had high levels of internalizing problem behavior (i.e., depression). Our developmental personality typology was also similar to other developmental typologies from adolescence into adulthood, as these typologies also included types comparable to Resilients, Undercontrollers, and Overcontrollers (Block, 1971; Morizot \& Le Blanc, 2003, 2005).

Almost four decades ago, Block (1971) made a case for incorporating normative developmental trends in personality typologies. Our results demonstrated that although the three types we found remain clearly distinguishable from one another from early to middle adolescence, they manifested themselves in slightly different ways across time. In other words, they displayed distinct patterns of normative development. These different developmental trajectories of the developmental types will now be discussed, trait by trait.

For Conscientiousness and Emotional Stability, all three types displayed a similar developmental trajectory with very little change across time. On these traits, differences between types were therefore present in a fairly consistent way from early to middle adolescence. With regard to Openness and Extraversion, differences between Overcontrollers and Resilients became larger across time. Openness has been shown to be positively related to success in job interviews (Caldwell \& Burger, 1998) and training proficiency (Salgado, 1997), while Extraversion has been related to occupational success once a job has been attained (Roberts, 1997). As such, our Overcontrollers seem to have moved towards a position that is at least somewhat less advantageous with regard to entering the labor market. However, the current study only focused on developmental trajectories of personality in early to middle adolescence. Future studies should investigate whether Overcontrollers regain a more advantageous position in late adolescence. 
We found increases in Agreeableness for Resilients, Undercontrollers, and Overcontrollers. The fact that Undercontrollers displayed increases while they initially had low scores on this trait might suggest that they outgrow their deficit. However, because Resilients and Overcontrollers also became more agreeable as they grow older, Undercontrollers retained their relatively disadvantageous position with regard to this trait when compared to Resilient and Overcontrollers. Our findings therefore suggest that Undercontrollers manifested themselves in somewhat different ways across adolescence as a function of normative development (i.e., changes that occur to a similar extent in most individuals in a sample; e.g., Roberts \& DelVecchio, 2000). Thereby, our results support Block's (1971) view that it is important to take normative development into account when studying developmental change in personality types, because mean-level changes do not necessarily indicate that one exhibits favorable changes in personality relative to others. Finally, there were no significant differences between types with regard to change rates of problem behavior. As such, Overcontrollers still displayed the highest levels of depression, and Undercontrollers still had the highest levels of delinquency by middle adolescence. This is in line with studies that identified personality types cross-sectionally (e.g., Akse et al., 2004; Asendorpf, 2003; Denissen et al., 2008; Hart et al., 2003; Robins et al., 1996).

Generally, adolescents exhibited only a limited amount of mean-level change. This might seem to contradict the general conception of adolescence as a period of "storm-andstress" (e.g., Arnett, 1999), and hence of rapid changes. However, a meta-analysis by Roberts, Walton, and Viechtbauer (2006) also revealed a limited amount of change in personality among adolescents. In line with these findings, Arnett (1999) previously concluded that adolescence only is a period of "storm-and-stress" for a minority of youth. As such, it is perhaps not too surprising that there generally only was a limited amount of mean-level change in personality traits.

The just mentioned studies that identified personality types cross-sectionally, consistently found that Resilients were the most prevalent. The present study had different results, as Overcontrollers were the most prevalent. The most likely reason of why we found more Overcontrollers than Resilients is related to the longitudinal methodology we applied to derive types. Using multiple measurement occasions to derive types is fundamentally different from using just one measurement occasion and can therefore yield different results. In fact, a previous study using a longitudinal typology (Block, 1971) also classified more individuals in types comparable to Overcontrollers, than in types comparable to Resilients. 
There were remarkable gender differences in the prevalence of developmental personality types. These gender differences were comparable to those previously demonstrated in studies using cross-sectional methodology to derive personality types (e.g., Dubas et al., 2002), as our results demonstrate that boys were more likely to be Undercontrollers and less likely to be Overcontrollers and Resilients. The latter finding, indicating that girls were more likely to be Resilients, suggests that the girls in our sample were more adjusted than the boys with regard to their personality profile.

\section{Limitations}

Although the current study presents one of the few attempts to revive Block's (1971) search for a developmental typology of personality, some limitations need to be recognized. First, the focus of the current study was restricted to early to middle adolescence. Although cross-sectionally derived Resilients, Undercontrollers, and Overcontrollers have been found in samples including children, adolescents, and adults (Asendorpf et al., 2001), it is not clear to what extent our developmental types are replicable in samples representing children or adults.

A second, but related, issue is that the current study only follows participants for a limited period of time (i.e., early to middle adolescence). It would be interesting to investigate developmental trajectories from adolescence into adulthood, like Block (1971) and Morizot and LeBlanc $(2003,2005)$ did. However, those studies applied less advanced statistical techniques and less frequent measurements, and employed smaller samples than the current study. Combining the strengths of our study and their studies would allow for an examination of a reliable developmental typological approach to life span personality development.

Third, the types we found in the current study mainly differ in levels of personality traits and much less in changes of these levels. In addition, it should be noted that the boundaries between personality types, which are thus most visible in the distinct levels they display, are fuzzy. Therefore, these types cannot be regarded as perfectly distinct entities (Asendorpf et al., 2001), but should instead be regarded as an attempt to explore the patterns of heterogeneity that tend to exist in personality development at the population level (Johnson et al., 2007).

A fourth limitation refers to our use of LCGA. This technique assigns individuals to classes based on their intercepts (i.e., initial levels) and slopes (i.e., change rates) (Nagin, 1999). LCGA does not necessarily assign more relative weight to one of the two, but uses variances around intercepts and growth factors to identify different developmental trajectories. In the current study, there was more variance around mean intercepts than around mean slopes. As a result, it should be noted that the impact of inter-individual differences in 
intercepts might have been larger than the impact of inter-individual differences in slopes in the classification of individuals in the several classes.

Finally, we only used adolescent self-reports to assess personality traits. The validity of self-reports could be limited due to social desirability. In addition, reported changes in personality might represent changes in the beliefs adolescents have about themselves (Robins, Fraley, Roberts, \& Trzesniewski, 2001). However, Robins, Noftle, Trzesniewski, and Roberts (2005) demonstrated that the beliefs young adults had about the way they changed, were quite accurate. The self-reports we used should provide a reliable estimate of personality of early adolescents, because Soto, John, Gosling, \& Potter (2008) demonstrated that self-reports already provided an accurate estimate of personality at age ten, and that the psychometrics of the Big Five did not change as adolescents grew older. As such, we believe that our use of adolescent self-reports is an appropriate method to assess adolescent personality change. Conclusion

Despite these potential limitations, the current study provides valuable insights into adolescent personality development. More specifically, we replicated the three adolescent personality types typically found in studies using cross-sectional methodology as developmental personality types. Resilients, Undercontrollers, and Overcontrollers exhibited different developmental trajectories, but remained clearly distinguishable from one another across time. 


\section{Chapter 5}

Longitudinal Associations Between Personality Profile Stability and Adjustment in College Students: Distinguishing Among Overall Stability, Distinctive Stability, and Within-Time Normativeness ${ }^{5}$

${ }^{5}$ Klimstra, T. A., Luyckx, K., Hale, W. W., Goossens, L., \& Meeus, W. H. J. (in press). Longitudinal associations between personality profile stability and adjustment in college students: Distinguishing among overall stability, distinctive stability, and within-time normativeness. Journal of Personality 


\begin{abstract}
In the present study, longitudinal associations of three aspects of personality profile stability (i.e., overall stability, distinctive stability, and within-time normativeness) with three adjustment measures (i.e., depressive symptoms, self-esteem, and delinquency) were examined, using four waves of longitudinal data on a Belgian college sample $(N=565)$. Longitudinal path models revealed strong longitudinal associations between adjustment and overall stability. Subsequent analyses showed that it was not the degree to which one's personality profile consistently diverges from the average personality profile within a population (i.e., distinctive stability) that was related to adjustment, but the degree to which a personality profile of an individual matches the average personality profile within the sample at a certain point in time (i.e., within-time normativeness). The current study thereby underscores the importance of distinguishing normativeness and distinctiveness when examining personality profile stability.
\end{abstract}




\subsection{Introduction}

Change and stability in personality can be operationalized in several ways (Block \& Robins, 1993). In the past, researchers have examined mean-level changes in personality traits (for an overview, see Roberts, Walton, \& Viechtbauer, 2006), changes in the rank-order of individuals on a specific trait (for an overview, see Roberts \& DelVecchio, 2000), and individual differences in changes on traits (e.g., Branje, van Lieshout, \& van Aken, 2004; De Fruyt et al., 2006; Roberts, Caspi, \& Moffitt, 2001). All these three aspects of change focus on changes in individual personality traits. However, these so-called variable-centered approaches tend to ignore that personality is in fact a "peculiar patterning of attributes within the single person" (Allport, 1954, p. 9). Hence, a fourth way to conceptualize change and stability of personality (i.e., personality profile stability) takes Allport's assertion into account by focusing on the stability of a constellation of personality traits within a person across time. Several studies found positive associations between personality profile stability and adjustment (Asendorpf \& van Aken, 1991; Block, 1971; Clausen, 1991; Donnelan, Conger, \& Burzette, 2007; Lönnqvist, Mäkinen, Paunonen, Henriksson, \& Verkasalo, 2008; Ozer \& Gjerde, 1989; Roberts et al., 2001).

However, recent work by Furr (2008) has revealed that profile stability cannot be regarded as a unitary construct. Instead, profile stability should be subdivided into several components: overall stability (i.e., "classic" profile stability), distinctive stability (i.e., the degree to which one's personality profile consistently diverges from the average personality profile within a population), and within-time normativeness (i.e., the degree to which one's personality profile is similar to the average or normative personality profile in a sample at a certain point in time). Until now, it is unclear which component of profile stability drives the effects of overall stability on adjustment. Consequently, the current study examines the longitudinal associations of all three aspects of personality profile stability (i.e., overall stability, distinctive stability, and within-time normativeness) with three adjustment measures (i.e., self-esteem, depressive symptoms, and delinquency). For this purpose, four-wave longitudinal data on a cohort of college students were analyzed.

\section{Personality Profile Stability: Measurement and Links with Adjustment}

Personality profile consistency is usually assessed by measuring profile similarity. For that purpose, an individual needs to be assessed on a set of personality traits on at least two subsequent measurement occasions. A rank-ordered set of personality traits on the first 
measurement occasion is then correlated with a rank-ordered set of the same personality traits on the second measurement occasion. The correlation between these two rank-ordered sets of personality traits, usually measured with $q$-correlations, reflects an individual's personality profile consistency (e.g., Furr, 2008; Roberts et al., 2001). Similar to Pearson test-retest correlations, $q$-correlations can range from -1 to 1 , with a higher $q$-correlation reflecting a more stable personality profile across time.

$Q$-correlations have been examined in several studies on personality development (Asendorpf \& van Aken, 1991; Block, 1971; Clausen, 1991; De Fruyt et al., 2006; Donnelan et al., 2007; Klimstra, Hale, Raaijmakers, Branje, \& Meeus, 2009; Lönnqvist et al., 2008; Ozer \& Gjerde, 1989; Roberts et al., 2001; Robins, Fraley, Roberts, \& Trzesniewski, 2001). These studies found average $q$-correlations ranging from .28 to .85 , with the higher average $q$ correlations found in studies examining personality consistency across shorter time intervals. In addition, two studies (Klimstra et al., 2009; Ozer \& Gjerde, 1989) revealed that $q$ correlations tend to increase with age from childhood to late adolescence. In general, all studies agreed that personality profiles were quite stable across time. Some of the previously mentioned studies (De Fruyt et al., 2006; Donnelan et al., 2007; Robins et al., 2001) have assessed personality consistency with a related index for profile similarity $\left(D^{2}\right)$ alongside $q$ correlations, but these studies merely demonstrated that both indices yielded the same conclusions. Further, $q$-correlations have recently been shown to be among the most reliable measures of profile similarity (McCrae, 2008).

Although the previously mentioned studies found that personality profiles are stable across time in a majority of individuals, they also found substantial inter-individual differences in personality consistency (with $q$-correlations across different studies ranging from -.95 to 1.00). For that reason, several studies have set out to investigate the meaning of these inter-individual differences in personality consistency, by examining associations of $q$ correlations with several adjustment measures. These studies typically found positive associations between personality profile stability on the one hand and desirable personality traits and competence on the other hand (Asendorpf \& van Aken, 1991; Block, 1971; Clausen, 1991; Lönnqvist et al., 2008; Ozer \& Gjerde, 1989). Two other studies (Donnelan et al., 2007; Roberts et al., 2001) obtained similar findings, as they found positive associations between profile stability on the one hand and Constraint (i.e., traditionalism, harm avoidance, and control) and low levels of Negative Emotionality on the other hand.

In sum, several studies have suggested that a stable personality profile is associated with positive adjustment. However, the aforementioned studies have treated personality 
profile stability as a unitary construct. Recently, Furr (2008) made a case for decomposing profile stability into several elements, as will be discussed in the next section.

Unpacking Personality Profile Stability: Overall Stability, Distinctive Stability, and WithinTime Normativeness

Furr (2008) recently outlined a framework that divides personality profile stability into several components, that is, overall stability, distinctive stability, and within-time normativeness. Overall stability refers to the within-person correlation between a rankordered set of traits on Time $t$ and a rank-ordered set of these same traits on Time $t+1$. Thus, overall stability is the type of profile stability that has been utilized in all studies mentioned above. However, when relying on such a global measure of profile stability, researchers are unable to distinguish between distinctive stability and within-time normativeness, which both have been hypothesized to capture different aspects of personality development across time (Furr, 2008).

Distinctive stability reflects the similarity between the distinctive or unique aspects of one's personality at Time $t$ and the distinctive aspects of the personality of that same person at Time $t+1$. More specifically, a person might rate him or herself higher on Time $t$ Neuroticism and lower on Time $t$ Conscientiousness than the average person in a sample does. If the same person's self-rated trait scores are again above average on Time $t+1$ Neuroticism and belowaverage on Time $t+1$ Conscientiousness, he or she shows a high level of distinctive stability (see Furr, 2008, for a similar example). Therefore, distinctive stability reflects whether an individual diverges from the average person (i.e., the sample norm) in a consistent manner across time.

A third aspect of profile stability is within-time normativeness. Within-time normativeness reflects the correlation between an individuals' personality profile and the average personality profile in a sample (i.e., a personality profile based on sample meanscores on all traits). For instance, if an individual ascribes a higher score to him- or herself on Agreeableness than on Conscientiousness, and the mean of the sample to which he or she belongs is also higher for Agreeableness than for Conscientiousness, this individual would show a high level of within-time normativeness. Because within-time normativeness reflects the correlation between an individual's personality profile and the normative profile for a certain sample at a certain point in time, within-time normativeness is captured through the use of a within-time correlation. Overall stability and distinctive stability, on the other hand, reflect an individual's personality consistency across time. As such, only one measurement 
occasion is needed to calculate within-time normativeness, whereas the calculation of overall stability and distinctive stability requires two measurement occasions.

Furr (2008) stated that the associations between personality profile stability and adjustment found in previous studies could very well be caused by the effect of within-time normativeness on adjustment, which he referred to as the normativeness problem. To deal with this normativeness problem, an approach in which several components of personality profile stability are related to adjustment is recommended. Such an approach would allow for investigating whether it is the cross-temporal stability of a personality profile that is associated with adjustment or, as hypothesized by Furr, whether it is within-time normativeness that causes these associations. As noted, both types of profile stability were not distinguished in previous studies.

\section{Aims of the Current Study}

The current study is the first to empirically decompose personality profile stability into overall stability, a normative component (i.e., within-time normativeness), and a distinctive component (i.e., distinctive stability), and to relate these three components of personality profile stability to adjustment (i.e., self-esteem, depressive symptoms, and delinquency). To infer the directionality of the relations between components of personality profile stability and adjustment, we simultaneously test whether these different components of personality profile stability predict levels of adjustment or whether adjustment predicts these components of personality profile stability. In the current study, the focus is mainly on depressive symptoms as a measure of adjustment, but we also briefly discuss how other indices of adjustment (i.e., self-esteem and delinquency) were related to overall profile stability, distinctive stability, and within-time normativeness. Our four-wave longitudinal design allows for examining whether obtained associations are replicated across time.

Based on the findings of the previous studies reviewed above, overall stability is expected to be positively associated with adjustment. The examination of the relations of distinctive stability and within-time normativeness with adjustment is more exploratory in nature, because the current study is the first to examine these associations. Thereby, the current study will provide a first attempt to shed light on Furr's (2008) proposition that it is the within-time normativeness component of overall personality profile stability that is responsible for the relations with adjustment that have typically been found in previous studies, and not so much the distinctiveness component. 


\subsection{Method}

\section{Participants and Procedure}

Participants were drawn from the Leuven Trajectories of Identity Development Study (L-TIDES; Luyckx, Goossens, \& Soenens, 2006), a 7-wave longitudinal study on college students from the faculty of Psychology and Educational Sciences at a large Belgian university. The first wave was collected at the end of 2002, and the Big Five of personality and the adjustment measures were assessed four times on an annual basis. Consequently, these four waves were used for the present manuscript. At Time 1, a total of 565 college students $\left(85.3 \%\right.$ female, $14.7 \%$ male; $M_{\text {age }}=18.63$ years $(S D=.61)$ participated. Permission to undertake this study was granted by the Institutional Review Board within the researchers' department. Participants signed a standard consent form before participating in the first wave of data collection. During the consent process, participants were informed that they could refuse or discontinue participation at any time. All students were assigned a unique code number to ensure confidentiality.

As in many longitudinal studies, data were missing at different time points for different participants. To minimize the bias associated with attrition and missing data, we used the expectation maximization (EM) algorithm to impute missing data (Little \& Rubin, 1987; Schafer \& Graham, 2002). Only individuals who participated in at least two out of four measurement waves $(N=485)$ were included. Overall, $12.31 \%$ of the data were missing. Participants with and without complete data were compared using Little's (1988) Missing Completely At Random (MCAR) test. This yielded a non-significant chi-square value $\left(\chi^{2}\right.$ $(120)=8.92, n s)$, suggesting that missing values could be reliably estimated.

\section{Measures}

Personality. As a measure of Costa and McCrae's (1992) Five Factor Model of personality, participants completed the Dutch version of the well-established 60-item NEO Five-Factor Inventory (NEO-FFI; Hoekstra, Ormel, \& De Fruyt, 1996). Cronbach's alphas across waves ranged from .87 to .89 for Neuroticism, from .80 to .82 for Extraversion, from .70 to .75 for Openness, from .68 to .72 for Agreeableness, and from .79 to .84 for Conscientiousness.

Depressive Symptoms. We used a 12-item shortened version of the Center for Epidemiologic Studies Depression Scale (CES-D; Radloff, 1977) to assess depressive symptoms. Items were scored on a 4-point Likert-type rating scale (ranging from "'seldom" 
to "most of the time or always") and refer to cognitive, somatic and psychological symptoms of depression. Participants were asked to indicate how often they experienced these symptoms during the past week. A sample item is "During the last week, I felt depressed". Cronbach's alphas across waves ranged from .88 to .89 .

Self-Esteem. General self-esteem was measured with the Rosenberg Self-Esteem Scale (RSES; Rosenberg, 1965). A sample item is "I feel that I have a number of good qualities". This scale contains 10 items scored on a 4-point Likert-type rating scale (ranging from " does not apply to me at all' to "applies to me very well'), Cronbach's alphas across waves ranged from .91 to .92 .

Delinquency. Delinquency was assessed with 11 items adapted from the Deviant Behavior Scale (DBS; Weinmann, 1992). Participants were asked to indicate on a 5-point Likert-type rating scale (ranging from "does not apply to me at all” to "applies to me very well'") the degree to which they engaged in deviant behaviors (such as shoplifting and destroying other people's property) during the past 6 months. Cronbach's alphas across waves ranged from .63 to .76 .

\section{Strategy of Analyses}

As a first step, overall personality stability was calculated with $q$-correlations. A $q$ correlation was calculated for each individual separately, by correlating a rank-ordered set of Big Five traits on one measurement occasion (e.g., T1) with a rank-ordered set of the same Big Five traits on the subsequent measurement occasion (e.g., T2). As there were four annual measurement waves of Big Five data available in the current study, this procedure resulted in three $q$-correlations of overall stability (i.e., for T1-T2, T2-T3, and T3-T4) for each individual.

Second, distinctive stability was calculated. This required us to center scores of all individuals on every Big Five Dimension on all measurement waves (Furr, 2008). That is, we subtracted the mean scores from the raw scores for each individual on every measurement occasion. Distinctive similarity was calculated with $q$-correlations by correlating a rankordered set of centered Big Five traits on one measurement occasion (e.g., T1) with a rankordered set of the same Big Five traits (which were again centered) on the subsequent measurement occasion (e.g., T2). Because there were four annual measurement waves of Big Five data available, we were able to calculate three $q$-correlations of distinctive stability (i.e., T1-T2, T2-T3, T3-T4) for each individual. 
Third, we calculated within-time normativeness coefficients for each individual on every measurement occasion. For that purpose, we first calculated sample mean scores for each Big Five trait on every measurement occasion. As a next step, we calculated within-time normativeness with $q$-correlations by correlating a rank-ordered set of the sample means on all Big Five traits with a rank-ordered set of Big Five traits for an individual. We followed this procedure for every person in the sample, and repeated it for each measurement occasion. As within-time normativeness coefficients represent within-time correlations, there were four measurements of within-time normativeness available (i.e., T1, T2, T3, and T4 within-time normativeness).

Longitudinal associations between adjustment and all three facets of Big Five profile stability were assessed with longitudinal path analyses by means of structural equation modeling in Mplus 4.2 (Muthén \& Muthén, 2007). Model fit was judged by assessing RMSEA's, CFI's, and TLI's. RMSEA's below .08, and CFI's and TLI's over .95 indicate adequate model fit; relatively lower RMSEA's, and higher CFI's and TLI's indicate a better fit when comparing models (Kline, 1998). Further, we used chi-square difference tests to compare model fits of nested models. To test whether stability paths of adjustment differed from those of the profile stability measures, we first constrained the stability paths of adjustment measures to be equal across waves, and constrained the stability paths of a profile stability measure to be equal across waves. In a subsequent step, we used chi-square difference tests to assess whether stability paths of adjustment differed from those of the profile stability measure. We used a similar procedure in order to test whether cross-paths from adjustment to a profile stability measure differed from those from the profile stability measure to adjustment.

\subsection{Results}

Overall stability, distinctive stability, and within-time normativeness were calculated for each individual. Descriptive statistics for these three aspects of profile stability and depressive symptoms on the various measurement waves are provided in Table 5.1.

To test the longitudinal associations of overall stability, distinctive stability, and within-time normativeness with depressive symptoms, we tested longitudinal path models. We first discuss the longitudinal associations between overall stability and depressive symptoms. Next, we move on to examine how the two components underlying overall profile 
stability (i.e., distinctive stability and within-time normativeness) are related to depressive symptoms.

Table 5.1

Means and Standard Deviations of Depressive symptoms and Three Components of Profile Stability

\begin{tabular}{|c|c|c|c|c|}
\hline \multirow[b]{2}{*}{ Measure } & T1 & $\mathrm{T} 2$ & T3 & $\mathrm{T} 4$ \\
\hline & $M(S D)$ & $M(S D)$ & $M(S D)$ & $M(S D)$ \\
\hline \multicolumn{5}{|l|}{ Adjustment } \\
\hline Depressive symptoms & $1.80(.51)$ & $1.72(.50)$ & $1.73(.47)$ & $1.70(.43)$ \\
\hline \multicolumn{5}{|l|}{ Profile Stability } \\
\hline Overall Stability & \multicolumn{2}{|c|}{$.72(.34)$} & \multicolumn{2}{|c|}{$.76(.36)$} \\
\hline Distinctive Stability & \multicolumn{2}{|c|}{$.59(.43)$} & \multicolumn{2}{|c|}{$.62(.45)$} \\
\hline Within-Time Normativeness & $.54(.49)$ & $.53(.50)$ & $.58(.49)$ & $.68(.44)$ \\
\hline
\end{tabular}

Note. Overall stability and distinctive stability are indexed by $q$-correlations between each pair of subsequent measurement waves. Because there were four measurement waves, there were three pairs of subsequent measurement waves, and hence three measurement occasions for overall stability and distinctive stability. $\mathrm{T}=$ Time.

\section{Overall Profile Stability}

The models that were used to examine relations between depressive symptoms and overall profile stability included all stability paths (e.g., T1 depressive symptoms -> T2 depressive symptoms, T1-T2 overall profile stability -> T2-T3 overall profile stability) and cross-paths (e.g., T1 depressive symptoms -> T1 - T2 overall profile stability). In the final model (see Figure 5.1), stability paths for depressive symptoms and overall profile stability, as well as cross-paths from overall profile stability to depressive symptoms, were freely estimated. Cross-paths from depressive symptoms to overall personality stability were constrained to be equal across waves, as chi-square difference tests demonstrated that adding such constraints did not affect model fit. The resulting model had an excellent fit to our data $\left(\chi^{2}(8)=15.19\right.$ (n.s.); CFI $=.99 ;$ TLI $=.96 ;$ RMSEA $=.04(90 \%$ C.I. $\left.=.04-.08)\right)$.

Figure 5.1 shows that both depressive symptoms and overall stability were reasonably stable over time. Chi-square difference tests revealed that stability paths were significantly stronger for depressive symptoms than for overall profile stability $(p<.001)$. In addition, all of the cross-paths from depressive symptoms to overall profile stability were significant, indicating that depressive symptoms was a negative predictor of overall profile stability. However, only one cross-path from overall profile stability to depressive symptoms reached significance. This cross-path (T1 - T2 overall profile stability -> T2 depressive symptoms) 
indicated that overall profile stability was a negative predictor of depressive symptoms.

Moreover, chi-square difference tests denoted that this cross-path did not significantly differ from the cross-paths from depressive symptoms to overall profile stability. The other crosspaths from overall profile stability to depressive symptoms were significantly weaker than the paths from depressive symptoms to overall profile stability $(p<.001)$.

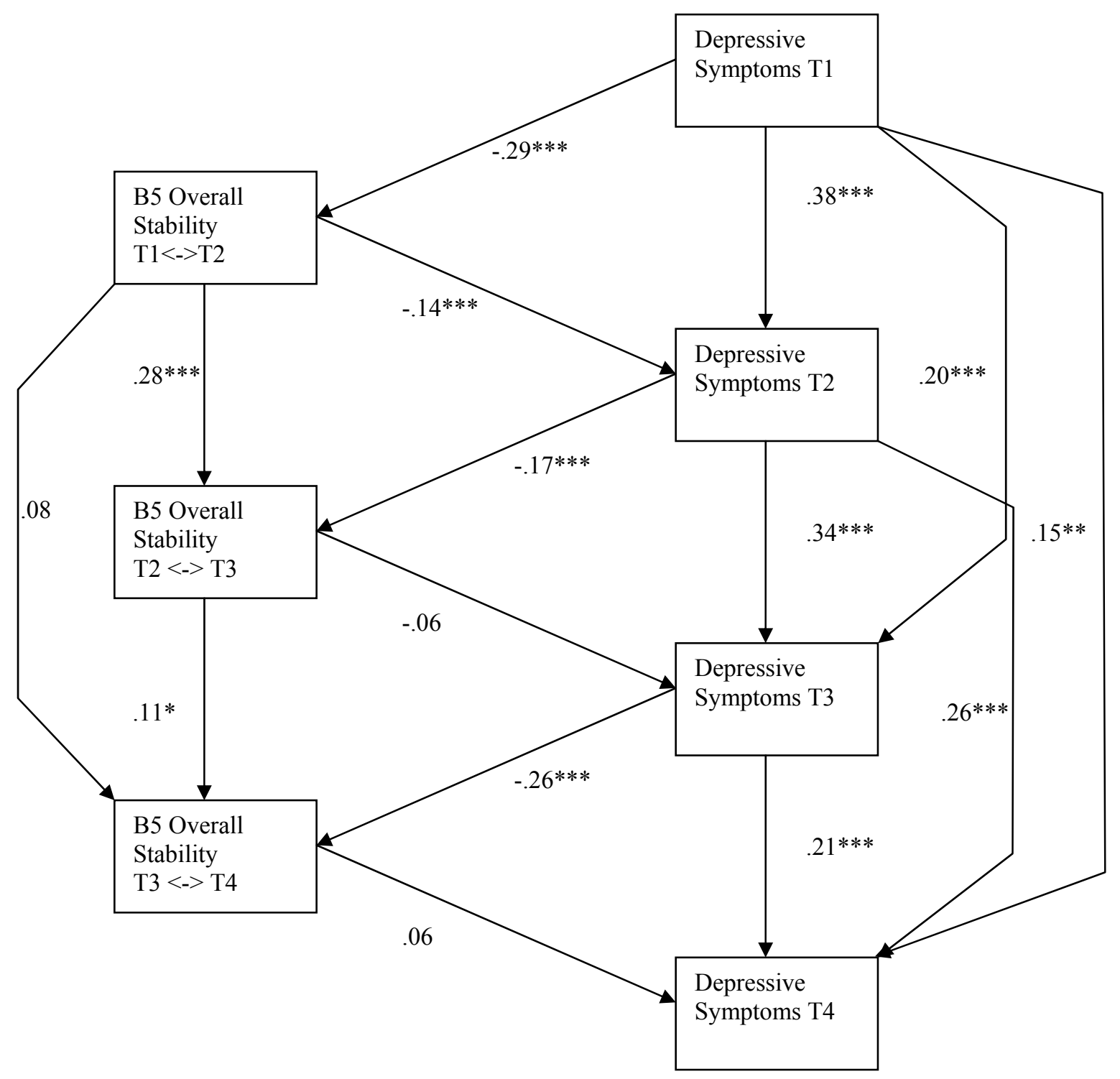

Figure 5.1. Longitudinal pathmodel for depressive symptoms and overall stability. 
Profile Stability and Adjustment

\section{Distinctive Profile Stability}

The model relating depressive symptoms to distinctive profile stability was similar to the model relating depressive symptoms to overall profile stability. Stability paths for both variables were freely estimated in this model. All cross-paths were constrained to be equal across waves, as adding such constraints did not affect model fit. The resulting model had an exceptional fit $\left(\chi^{2}(10)=7.13\right.$ (n.s.); CFI $=1.00 ; \mathrm{TLI}=1.02$; RMSEA $=.00(90 \%$ C.I. $=.00$ $.04))$ and is displayed in Figure 5.2.

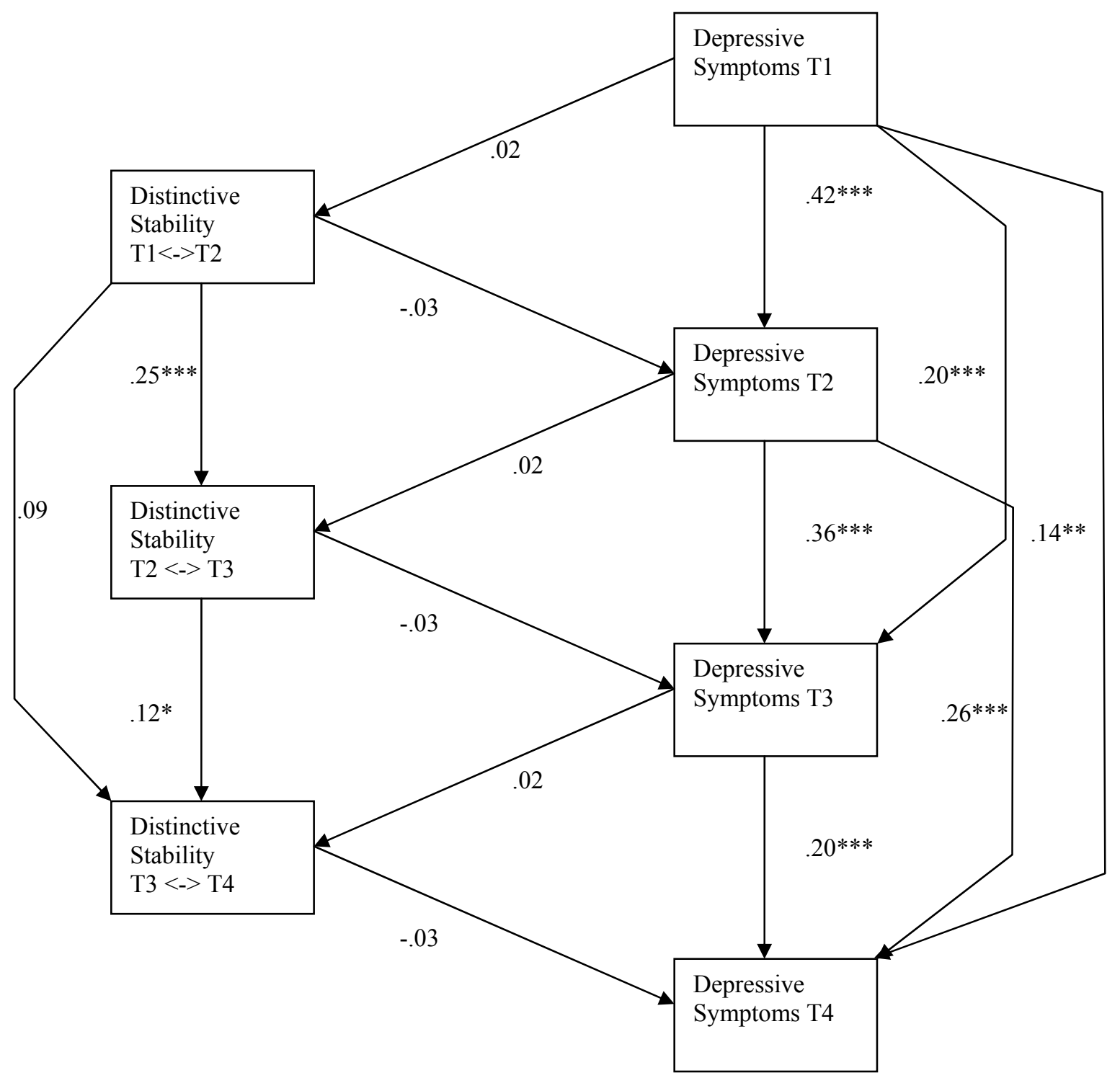

Figure 5.2. Longitudinal pathmodel for depressive symptoms and distinctive stability. 
Both constructs were reasonably stable across time, but the stability paths of depressive symptoms were significantly stronger than those for distinctive stability $(p<.001)$. None of the cross-paths reached significance.

\section{Within-Time Normativeness}

We used longitudinal path models including stability paths (e.g., T1 depressive symptoms -> T2 depressive symptoms), within-time correlations (e.g., T1 within-time normativeness <-> T1 depressive symptoms), and cross-paths (e.g., T1 depressive symptoms $>$ T2 within-time profile normativeness). Stability paths for depressive symptoms were freely estimated, similar to cross-paths and correlations between depressive symptoms and withintime personality profile normativeness. Stability paths of within-time normativeness were constrained to be equal across time, as adding such constraints did not affect model fit. The resulting model (displayed in Figure 5.3) had an excellent fit $\left(\chi^{2}(10)=7.16\right.$ (n.s.); CFI $=$ 1.00; TLI $=1.01 ;$ RMSEA $=.00(90 \%$ C.I. $=.00-.04))$.

As Figure 5.3 shows, within-time profile normativeness was more stable across time than depressive symptoms $(p<.001)$. Depressive symptoms and within-time profile normativeness were strongly related to each other at the initial level, and there was also evidence for correlated change. In addition, two out of three cross-paths from depressive symptoms to within-time profile normativeness reached significance. Both paths revealed that higher levels of depressive symptoms on one measurement occasion predicted relative decreases of within-time normativeness on the subsequent measurement wave. Two out of three cross-paths from within-time normativeness to depressive symptoms reached significance. These cross-paths indicated that higher levels of within-time normativeness predicted relative decreases in depressive symptoms. Overall, the cross-paths from withintime normativeness to depressive symptoms turned out to be equally strong as the cross-paths from depressive symptoms to within-time normativeness. 


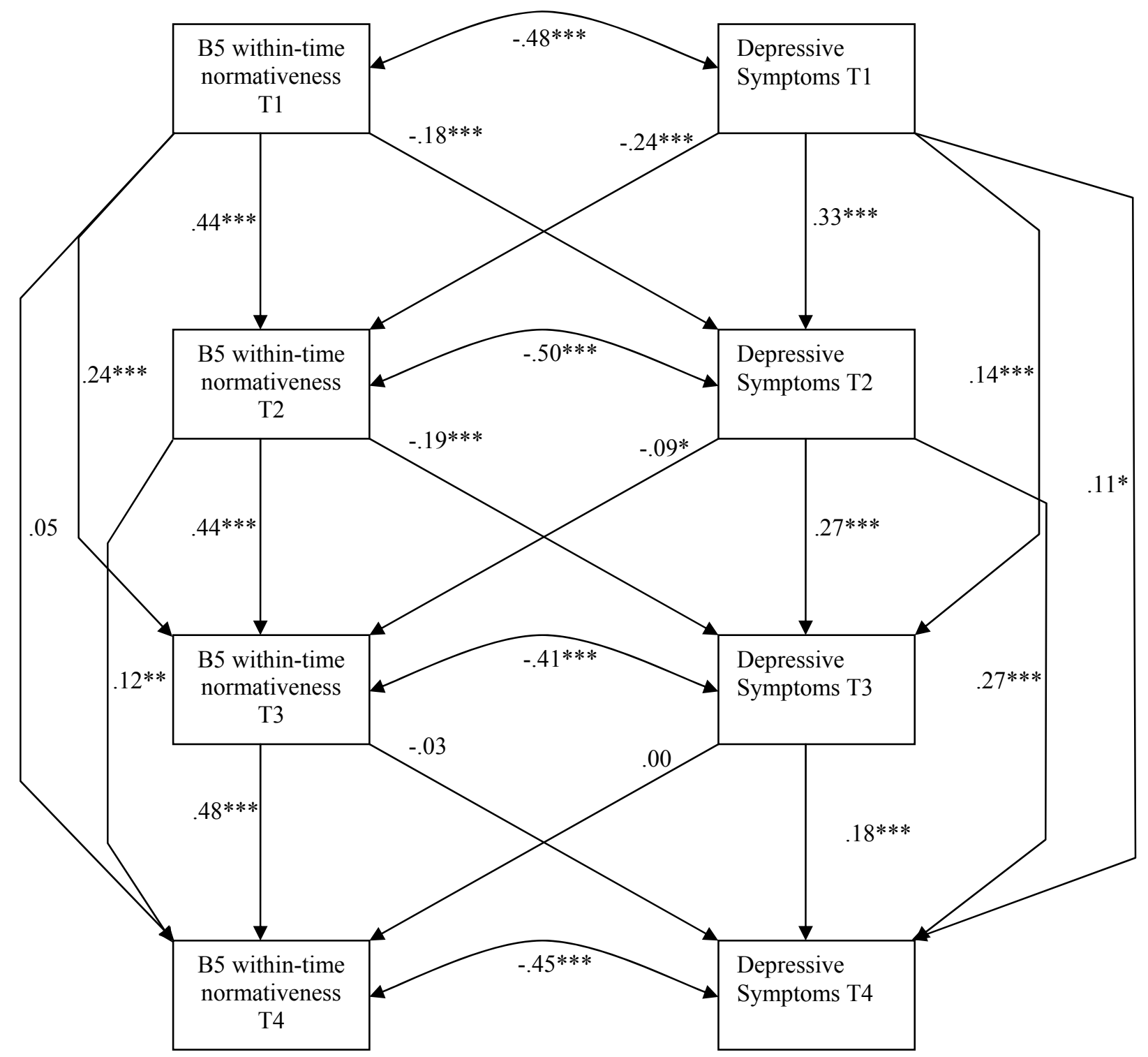

Figure 5.3. Longitudinal pathmodel for depressive symptoms and within-time normativeness.

\section{Ancillary Analyses with Self-Esteem and Delinquency}

To examine whether our findings could be generalized across a wider range of adjustment measures, we ran a series of models to test the longitudinal associations between self-esteem and delinquency on the one hand, and overall stability, distinctive stability and within-time normativeness on the other hand. The longitudinal associations obtained with these models are depicted in Table 5.2. To facilitate comparisons with the depression models, the standardized estimates of those models are also shown in Table 5.2. 
Table 5.2

Longitudinal Associations of Overall Stability, Distinctive Stability, and Within-Time

Normativeness with Self-Esteem and Delinquency (Standardized Estimates)

\begin{tabular}{cccc}
\hline & Depression & Self-Esteem & Delinquency \\
\hline Overall Stability: Cross-Paths & & & $-.24^{* * * *}$ \\
Adj T1 -> OS T1-T2 & $-.29^{* * *}$ & $.16^{* * *}$ & $-.07^{* * *}$ \\
Adj T2 -> OS T2-T3 & $-.17^{* * *}$ & $.24^{* * *}$ & $-.09^{* * *}$ \\
Adj T3 -> OS T3-T4 & $-.26^{* * *}$ & .04 & .00 \\
OS T1-T2 -> Adj T2 & $-.14^{* * *}$ & .01 & .00 \\
OS T2-T3 -> Adj T3 & -.06 & $-.08^{*}$ & .00 \\
OS T3-T4 -> Adj T4 & -.06 & & \\
Distinctive Stability: Cross-Paths & & $-.06^{*}$ & -.02 \\
Adj T1 -> DS T1-T2 & .02 & $-.06^{*}$ & -.01 \\
Adj T2 -> DS T2-T3 & .02 & $-.05^{*}$ & $.10^{*}$ \\
Adj T3 -> DS T3-T4 & .02 & .00 & $.09^{*}$ \\
DS T1-T2 -> Adj T2 & -.03 & .00 & -.02 \\
DS T2-T3 -> Adj T3 & -.03 & .00 & -.02 \\
DS T3-T4 -> Adj T4 & -.03 & &
\end{tabular}

Within-Time Normativeness:

Within-Time Correlations

Adj T1<-> WN T1
Adj T2<-> WN T2
Adj T3 <-> WN T3
Adj T4 <-> WN T4

Within-Time Normativeness: Cross-

Paths

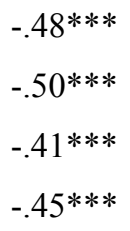

$.58 * * *$

$44 * * *$

$46^{* * * *}$

$.55 * * *$

$-.16^{* * *}$

$-.10 * * *$

$-.14 * * *$

$-.16^{* * *}$

Adj T1 -> WN T2
Adj T2 -> WN T3
Adj T3 -> WN T4
WN T1 -> Adj T2
WN T2 -> Adj T3
WN T3 -> Adj T4

$-.24 * * *$
$-.09 *$
.00
$-.18 * * *$
$-.19 * * *$
.03

$.31 * * *$

$-.13 * * *$

$.10 * *$

$-.10 * * *$

$.10 * *$

$-.10 * * *$

.04

$-.04 *$

$.17 * * *$

$-.05 *$

.05

$-.05 *$

Note. Adj = Adjustment (i.e., Self-esteem or Delinquency); OS= Overall Stability; DS = Distinctive Stability; $\mathrm{WN}=$ Within-Time Normativeness; $\mathrm{T}=$ Time. Stability paths were estimated, but omitted from this table. All models depicted in this table had an excellent fit (CFI's were all 1.00; RMSEA's ranged from .00 to .03).

Estimates for stability paths, exact model fits, and other details about the models can be obtained from the first author upon request. * $p<.05 . * * p<.01 . * * * p<.001$.

As shown in Table 5.2, our analyses with self-esteem and delinquency as indicators of adjustment produced similar results as our analyses with depressive symptoms did. That is, adjustment (indicated by higher levels of self-esteem and lower levels of delinquency) was 
positively related to overall stability. Similar to our results for depressive symptoms, selfesteem and delinquency were almost unrelated to distinctive stability, while both were strongly associated with within-time normativeness. More specifically, within-time normativeness was negatively related to delinquency, and positively associated with selfesteem.

\subsection{Discussion}

The main purpose of the present study was to examine which component of personality profile stability is responsible for the commonly reported associations with adjustment (Asendorpf \& van Aken, 1991; Block, 1971; Clausen, 1991; Donnelan et al., 2007; Lönnqvist et al., 2008; Ozer \& Gjerde, 1989; Roberts et al., 2001). To achieve this objective, we examined longitudinal associations of three components of personality profile stability (i.e., overall stability, distinctive stability, and within-time normativeness; Furr, 2008) with three adjustment measures (i.e., self-esteem, depressive symptoms, and delinquency) in a sample of college students.

\section{Longitudinal Associations of Adjustment with Three Components of Personality Profile Stability}

Our results indicated that self-esteem, depressive symptoms, and delinquency all had strong longitudinal associations with overall Big Five profile stability. Paths from the adjustment measures to overall profile stability were stronger than the paths in the opposite direction. If overall stability is considered to be a unitary construct, the appropriate conclusion would be that higher levels of well-being and lower levels of internalizing and externalizing problem behaviors seem to foster a more consistent personality profile. This conclusion would be in line with Roberts et al. (2001), who noted that maturity is related to changes towards a desirable endpoint and that the likelihood of personality change diminishes as individuals come closer to that endpoint. However, as previously mentioned, Furr (2008) stated that associations between overall stability and adjustment are likely to be confounded with normativity (i.e., the associations of an individual's personality profile with the personality profile of the average person within a sample), and proposed a paradigm in which normativity and distinctive profile stability (i.e., the degree to which one's personality profile diverges from the average personality profile in a consistent manner) were distinguished from one another. In subsequent sets of analyses, we therefore assessed the longitudinal associations of distinctive profile stability and within-time profile normativity with adjustment. 
Longitudinal associations between distinctive personality profile stability and adjustment were, at best, weak. Only some marginally significant paths between delinquency and self-esteem on the one hand and distinctive stability on the other hand were found. With respect to depressive symptoms, there were no associations with distinctive profile stability. Therefore, it would appear that the distinctiveness component of overall profile stability could not account for the strong associations of overall profile stability with adjustment.

In line with Furr's (2008) predictions, our analyses showed that the normativeness component of overall profile stability was strongly associated with adjustment. There were strong concurrent associations of all three adjustment measures with within-time normativeness, and changes in adjustment and within-time normativeness were also strongly associated. These associations suggested that increases in adjustment (i.e., increases in selfesteem, and decreases in depressive symptoms and delinquency) were associated with changes in personality that caused an individual's personality profile to become more similar to the average ("normative") profile within the sample. Adjustment and normativeness of personality profiles were not only associated with one another, they also predicted each other over time. There were paths from adjustment to normativeness, but also from normativeness to adjustment. Two of the three adjustment measures (i.e., self-esteem and delinquency) turned out to be stronger predictors of within-time profile normativeness than within-time profile normativeness was for these adjustment measures. Depressive symptoms and withintime profile normativeness turned out to be equally predictive of one another.

In sum, our analyses revealed that the longitudinal associations between personality profile stability and adjustment that have been reported in previous studies (Asendorpf $\&$ van Aken, 1991; Block, 1971; Clausen, 1991; Donnelan et al., 2007; Lönnqvist et al., 2008; Ozer $\&$ Gjerde, 1989; Roberts et al., 2001) are quite likely to be caused by the effect that withintime normativeness has on adjustment. At least in the present study, distinctive stability and adjustment were mostly unrelated to one another, whereas normativeness of personality profiles and adjustment were strongly related. Thus, previous studies could follow Furr's (2008) recommendations and re-analyze their data by distinguishing among overall profile stability, distinctive profile stability, and within-time normativeness.

Although distinctive stability was almost unrelated to adjustment, it still constitutes an important aspect of personality profile stability. Table 5.1 showed that distinctive stability is quite high among early adults, ranging from .59 to .62 in the current study. These relatively high figures suggest that relative differences in personality profiles of early adults are rather consistent across time. For that reason, we can conclude that inter-individual differences in 
personality among early adults are not only quite stable with regard to traits (Costa \& McCrae, 1994), but also with regard to profiles. Given these high figures of distinctive stability in early adults, it would be interesting to examine age-related increases in distinctive stability. Age-related increases in overall stability have previously been reported (e.g., Klimstra et al., 2009; Ozer \& Gjerde, 1989), but there have been no studies examining increases in distinctive stability yet.

Because the effect of overall stability on adjustment appears to be caused by the effect of normativeness on adjustment, Roberts et al.'s (2001) proposition that maturity is related to changes towards a desirable endpoint and that the likelihood of personality change diminishes as individuals come closer to that endpoint needs to be reformulated with regard to personality profile stability. Based on our results, changes towards maturation (indicated by increases in self-esteem, and decreases in depressive symptoms and delinquency) are related to personality changes that reflect increases in the degree to which an individual's personality profile matches the profile of the average person in a population. In more recent work by Roberts et al. (2006), a similar mechanism is described. They noted that young adults (e.g., college students) are faced with an increasing pressure to adhere to social role expectations. Individuals who do not comply with these role expectations, are likely to face withdrawal of social approval, which, in turn, can lead to negative consequences such as losing a job. If individuals do meet social expectations, they are likely to receive greater levels of acceptance and social support (Roberts et al., 2006). Roberts et al. (2006) described increases in Conscientiousness and Emotional Stability as a movement towards greater compliance with social role expectations. Based on the present findings, we propose that an increase in normativity (i.e., the degree to which one's personality profile matches the average personality profile within a sample) could reflect a similar mechanism. As such, it is not surprising that people with a personality profile that matches social norms reflect higher levels of adjustment then individuals with a profile that diverges from these norms.

\section{Strengths and Limitations of the Current Study}

Taken together, our study has several strengths. The main strength is that it is the first study to examine the longitudinal associations of adjustment with three separate components of personality profile stability: overall profile stability, distinctive profile stability, and withintime normativeness (Furr, 2008). A second strength is the inclusion of four measurement 
occasions. The inclusion of multiple measurement occasions allowed us to demonstrate that associations did not just occur randomly, but could consistently be replicated across time. A third strength was our inclusion of three different adjustment measures. This allowed us to demonstrate that our findings were not specific to negative adjustment in the internalizing domain (i.e., depressive symptoms), but could be generalized to positive adjustment (i.e., selfesteem) in the internalizing domain and negative adjustment in the externalizing (i.e., delinquency) domain.

Notwithstanding the strengths of the current study, several limitations need to be recognized. First, women comprised $85 \%$ of the sample in the current study. Therefore, the generalizability of our results needs to be confirmed with samples with more balanced gender distributions. Second, our sample only included college students. Therefore it is not clear to what extent our results are descriptive of the relation between personality consistency and adjustment in the general population of young adults. For that reason, future studies should seek to replicate our findings in a non-college sample.

The fact that we relied solely on self-reported questionnaires can be considered a third limitation. Especially for personality, other-reported questionnaires have been shown to lead to slightly different conclusions in studies on mean-level change when compared to selfreport questionnaires (Branje, van Lieshout, \& Gerris, 2007). Although other-reports are not necessarily more accurate than self-reports when judging personality (Funder, 1995), adding other-reports would provide valuable insights as to whether individuals who are judged inconsistent or non-normative by others, judge themselves in a similar way. In addition, such studies could assess whether self-reported and other-reported components of personality profile stability have a differential effect on, or are differentially affected by adjustment. Therefore, future studies should ideally assess the relations between both self-reported and other-reported personality profile stability components and adjustment in a single longitudinal design.

Despite these potential limitations, the present study provides a substantial contribution to the current knowledge on personality profile stability as our findings strongly suggest that it is indeed important to follow Furr's (2008) recommendations to distinguish among different components of personality profile stability. We demonstrated that the stability of the distinctive components of one's personality profile is almost unrelated to adjustment, which suggests that it is not this component of overall profile stability that seems to drive the links with adjustment. Instead, the degree to which an individual's personality profile matches the personality profile of an average person in a sample is strongly related to 
Profile Stability and Adjustment

adjustment and would therefore appear to be the most likely cause of the associations of overall profile stability and adjustment. More specifically, our results reveal that individuals who display a personality profile that better matches the personality profile of the average person in a population have higher levels of self-esteem, and lower levels of depressive symptoms and delinquency. 


\section{Chapter 6}

Too Early or Too Late: Hypermaturity and Immaturity of Personality in Adolescent Boys and Girls ${ }^{6}$

${ }^{6}$ Klimstra, T. A., Hale, W. W., Raaijmakers, Q. A. W., \& Meeus, W. H. J. (2010). Too early or too late: Hypermaturity and immaturity of personality in adolescent boys and girls. Manuscript submitted for publication. 


\begin{abstract}
Five-year longitudinal data on a cohort of early to middle adolescents $(N=923)$ and a cohort of middle to late adolescents $(N=390)$ were used to examine the consequences of hypermaturity (i.e., 12-year-olds with a personality profile resembling the profile of an average 20-year-old) and immaturity (i.e., 20-year-olds with a personality profile resembling the profile of an average 12-year-old) of personality. Analyses revealed that girls with high levels of hypermaturity exhibited high levels of internalizing problem behavior and conflict with parents, while hypermaturity in boys was only associated with internalizing problems. Both boys and girls with high levels of immaturity had low levels of anxiety, but immature girls also had high levels of externalizing problems.
\end{abstract}




\subsection{Introduction}

Adolescence is generally regarded as the formative period in a person's life.

Individuals undergo tremendous changes towards maturation, both physically (e.g., Petersen, Crockett, Richards, \& Boxer, 1988) and psychosocially (e.g., Erikson, 1950). In the physical domain, an individual grows from a child posture towards an adult posture (Petersen et al., 1988). Psychosocially, adolescents gradually leave their parents' teachings behind in order to start developing their own set of ethics, ideals, and identifications (Erikson, 1950). This process is, for example, reflected in adolescents dealing with identity issues in an increasingly more mature manner (Meeus, Iedema, Helsen, \& Vollebergh, 1999), while they also develop their own cultural orientations (Vollebergh, Iedema, \& Raaijmakers, 2001). In the relational domain, there are also prominent changes. Adolescents tell their parents less about their activities, and parents exert less control on adolescents as they grow older (Keijsers, Frijns, Branje, \& Meeus, 2009). At the same time, parents are gradually replaced by peers as the primary source of support. Thus, the role of parents diminishes, while relationships with peers become increasingly more salient and important throughout adolescence (De Goede, Branje, Delsing, \& Meeus, 2009; Furman \& Buhrmester, 1992).

All these physical and psychosocial changes towards maturation are likely to go handin-hand with changes in personality traits. Over the last two decades, a majority of researchers has come to agree that the higher-order structure of personality can be subsumed in five broad traits: the Big Five (Caspi, Roberts, \& Shiner, 2005). The Big Five traits are Extraversion (dominance and activity in interpersonal situations), Agreeableness (willingness to maintain positive and reciprocal relationships with others), Conscientiousness (organizational and motivational aspects of one's behavior), Emotional Stability (the ability to cope with negative emotions), and Openness to Experience (reflecting how a person deals with new information at a personal and experiential level) (Caspi et al., 2005; McCrae \& Costa, 1987). One metaanalysis has revealed increases in mean-levels of personality traits (Roberts, Walton, \& Viechtbauer, 2006), and another meta-analysis, on rank-order stability, demonstrated that inter-individual differences become more set with age (Roberts \& DelVecchio, 2000). In a recent empirical study, Klimstra, Hale, Raaijmakers, Branje, and Meeus (2009) had similar findings. In addition, they found substantial gender differences in mean-level change and rank-order stability, with girls displaying high mean-levels and rank-order stability at an earlier age than boys did. 
Hypermaturity and Immaturity

In sum, it appears that inter-individual differences in personality traits (i.e., rank-order stability) are quite stable whereas mean-levels of personality traits increase. This suggests that the mean-level changes that occur apply to a majority of individuals in the population. Meanlevel changes that apply to a majority of individuals in a population are referred to as normative changes, and are thought to reflect general maturation processes (e.g., Roberts et al., 2006). It is, however, unclear what the consequences are when one is ahead or lagging behind on these normative patterns of personality change. In other words, it is unclear what the consequences of "off-time adolescent personality development" are. We will now briefly discuss the consequences of off-time adolescent development in general, and then move on to the measurement as well as the possible consequences of off-time development with regard to adolescent personality.

\section{Off-Time Adolescent Development: Concepts and Consequences}

There are basically two types of off-time adolescent development. Adolescents can mature much earlier (i.e., hypermaturity) or much later (i.e., immaturity) than their peers. Little is known about hypermaturity and immaturity of personality, but there is a substantial amount of literature on early and late pubertal timing (i.e., being off-time when compared to peers with regard to physical maturation; e.g., Petersen et al., 1988).

Early pubertal timing in boys and girls, and late pubertal timing in boys is associated with a wide range of internalizing (e.g., anxiety and depression) and externalizing (e.g., delinquency) problems (for an overview, see Graber, 2003). Late pubertal timing in girls has not been associated with problem behavior (Graber, 2003). Thus, early and late pubertal timing are associated with poor mental health. This raises the question of exactly why this is. Eccles et al. (1993) suggested that the effects of early and late pubertal timing might be explained by a poor stage-environment fit. They proposed that a mismatch between the developmental level of individuals and their environment could lead to increases in adolescent problem behavior. They further suggest that adolescents who are either too mature or too immature for their chronological age are likely to elicit responses from teachers, parents and peers that do not match their developmental needs. This, in turn, may be associated with adverse consequences for psychological well-being.

It is quite likely that this mechanism may also be applicable to the field of personality development. For example, Roberts et al. (2006) described personality development as a function of shifting role expectations. They ascribed increases in Conscientiousness, Emotional Stability, and the social dominance aspect of Extraversion to early adults' 
adjustment to universal tasks of social living (e.g., establishing a career, starting a family). If individuals do not reflect appropriate levels on these personality traits, they are likely to face lower levels of social approval and social reinforcement. Consequently, they could become less-adjusted. As such, Roberts et al. (2006) also described a poor fit between an individuals' personality and his or her social environment as a risk factor for poor psychosocial health. Unfortunately, the consequences of off-time personality development have not been empirically examined until now. The present study provides a first attempt to examine the consequences of off-time personality development, by examining the correlates of hypermaturity and immaturity of personality.

\section{Hypermaturity and Immaturity of Personality}

Measuring off-time pubertal developmental (i.e., reaching a milestone such as menarche substantially earlier of later when compared to peers) with regard to physical characteristics is relatively straightforward, as it is typically assessed with either one indicator or a set of highly correlated indicators (e.g., Graber et al., 1997; Petersen et al., 1988). With regard to the specific domain of personality, this is somewhat more complicated as personality is typically subsumed under the traits of the Big Five. Therefore, one could assess whether personality development is on-time or off-time for every single dimension separately, but this would not make much sense because personality should be perceived as a particular configuration of traits within a single person (Allport, 1954). Recently, Furr (2008) came up with a solution to this problem by proposing a way to calculate the "normativity" of personality profiles with $q$-correlations.

Normativity of personality profiles as assessed with $q$-correlations represents the within-person correlation of an individuals' rank-ordered set of personality traits with a rankordered set representing a normative personality profile (i.e., a profile based on sample mean scores on all traits). As such, normativity of personality profiles reflects the degree to which an individual's personality profile matches the normative personality profile within a particular population (e.g., an adolescent population). The $q$-correlation reflecting normativity of personality profiles can range from -1 (i.e., a perfect mismatch between an individual's profile and the normative profile) and 1 (i.e., a perfect match between an individual's profile and the normative profile). Typically, one would, for example, compare the personality profile of an individual college freshman to the personality profile of an average college freshman (see Klimstra, Luyckx, Hale, Goossens, \& Meeus, in press). This kind of normativeness is referred to as within-time normativeness, and has been shown to be 
negatively correlated with problem behavior symptoms (Klimstra et al., in press). Thus, individuals with a personality profile that deviates from the average profile, tend to display higher levels of problem behavior symptoms.

In addition to within-time normativeness, Furr (2008) also briefly mentioned an appealing opportunity to calculate two types of across-time normativeness. First, one could assess the degree to which a younger aged individual's personality profile matches the normative profile of an older age cohort. Second, it is possible to calculate the degree to which an older aged individual's profile matches the normative profile of a younger age cohort. These two types could be indicative of developmental hypermaturity and immaturity of personality, respectively. Hypermaturity would indicate that one is ahead on the normative patterns of personality change, whereas immaturity would indicate that one is lagging behind.

The aim of the current study is to explore these concepts of developmental hypermaturity and immaturity of personality. For this purpose, we will employ five-annual wave longitudinal data on a cohort of early to middle adolescents who were 12 years of age on average at the first measurement occasion, and a cohort of middle to late adolescents who were 16 years of age on average at the first measurement occasion. As such, an age range from 12 to 20 years is available. Hypermaturity will be operationalized as the degree to which a profile of a 12-year-old (i.e., the T1 profile of an early to middle adolescent) matches the normative profile of a 20 -year-old (i.e., based on means of Big Five traits of middle to late adolescents at T5). Immaturity reflects the degree to which a profile of a 20 -year-old (i.e., the T5 profile of a middle to late adolescent) matches the normative profile of a 12-year-old (i.e., based on means of Big Five traits of early to middle adolescents a T1). Thus, we will use data from two cohorts to determine hypermaturity of personality for 468 early to middle adolescent boys and 455 early to middle adolescent girls, and immaturity of personality for 169 middle to late adolescent boys and 221 middle to late adolescent girls.

To examine the correlates of hypermaturity and immaturity of personality, we will correlate the indices reflecting these types of off-time personality development with three indicators of adolescent "storm-and-stress" problem behaviors (Arnett, 1999): (a) mood disruptions (i.e., anxiety and depression), (b) risk behavior (i.e., delinquency), and (c) conflict with parents (i.e., frequency of conflicts). Because within-time normativeness of personality (i.e., the degree to which one's personality profile matches the profile of same-aged peers; see Furr, 2008; Klimstra et al., in press) has been shown to be strongly associated with problem behavior symptoms, we will statistically control for the effects of within-time normativeness when examining the effects of hypermaturity and immaturity of personality. 
Until now, there have been no studies on off-time personality development. Although it is unclear to what extent findings with regard to pubertal timing can be generalized to offtime personality development, it is at least clear that being off-time in other areas of adolescent development goes together with internalizing and externalizing problem behavior symptoms. As such, we also expect hypermaturity and immaturity of personality to go together with poor psychosocial adjustment. Because previous studies found substantive gender differences in adolescent personality (e.g., Klimstra et al., 2009), we will account for such differences by running analyses for boys and girls, separately.

\subsection{Method}

\section{Participants}

For this study, we used data from an ongoing longitudinal research project on Conflict And Management Of Relationships (CONAMORE; Meeus et al., 2006). The longitudinal sample consisted of 1,313 participants divided into an early to middle adolescent cohort $(n=$ $923 ; 70.3 \%)$ who were 12.4 years of age on average $(S D=.59)$, and a middle to late adolescent cohort $(n=390 ; 29.7 \%)$ with an average age of 16.7 years $(S D=.80)$ during the first wave of measurement. The early to middle adolescent cohort consisted of 468 boys (50.7\%) and 455 girls (49.3\%), and the middle to late adolescent cohort consisted of 169 boys (43.3\%) and 221 girls (56.7\%). Because both age groups were assessed during five measurement waves, a total age range from 12 to 20 years was available. In this particular study, we used the first measurement wave of the early to middle adolescent cohort, and the fifth (and final) measurement wave of the middle to late adolescent cohort.

Missing values were estimated in SPSS, using the EM-procedure. Across waves $4.94 \%$ of the data was missing. Little's Missing Completely At Random Test (Little, 1988) revealed a normed $\chi^{2}\left(\chi^{2} / \mathrm{df}\right)$ of 1.20 which according to guidelines by Bollen (1989) indicates a good fit between sample scores with and without imputation.

\section{Procedure}

The participating adolescents were recruited from various high schools in the Province of Utrecht, The Netherlands. Participants and their parents received an invitation letter, describing the research project and goals, and explaining the possibility to decline from participation. More than $99 \%$ of the approached high school students decided to participate. All participants signed the informed consent form. The questionnaires were completed at the 
Hypermaturity and Immaturity

participants' own high school, during annual assessments. Confidentiality of responses was guaranteed. Verbal and written instructions were offered. The adolescents received $€ 10$ (approximately US \$14) as a reward for every wave they participated in.

\section{Measures}

Personality. Personality was assessed with the shortened Dutch version of Goldberg's Big Five questionnaire (Gerris et al., 1998; Goldberg, 1992). In this instrument, a 7-point likert scale, with a response format ranging from 1 (completely untrue) to 7 (completely true), is used to assess five personality dimensions: Extraversion, Agreeableness, Conscientiousness, Emotional Stability, and Openness to Experience. All dimensions are measured with 6 items each, such as: talkative (Extraversion), sympathetic (Agreeableness), systematic (Conscientiousness), worried (Emotional Stability), and creative (Openness to Experience). Reliability was high across waves, as Cronbach's Alphas for early to middle and middle to late adolescents ranged from .76 to .81 for Extraversion, from .80 to .88 for Agreeableness, from .81 to .92 for Conscientiousness, from .79 to .85 for Emotional Stability, and from .74 to .79 for Openness to Experience.

Anxiety. The Screen for Child Anxiety Related Emotional Disorders (SCARED) was used to measure anxiety symptoms. The SCARED is a self-report questionnaire, which is used to measure symptoms of DSM-IV linked anxiety disorders in children and adolescents. It has been shown to be a reliable and valid measure (Birmaher et al., 1997; Muris, Merckelbach, Van Brakel, \& Mayer, 1999; Muris \& Steerneman, 2001). Its factor structure has consistently been replicated among early and middle adolescents, and boys and girls (Hale, Raaijmakers, Muris, \& Meeus, 2005). The SCARED consists of 38 items and contains five subscales: panic disorder symptoms (13 items), social anxiety symptoms (4 items), separation anxiety symptoms ( 8 items), generalized anxiety symptoms ( 9 items) and school phobia symptoms (4 items). In this study, the composite anxiety score is measured, hence the subscales are not investigated separately. Sample items include 'When frightened, it is hard to breathe', 'I don't like to be with people I don't know', 'I get scared when I sleep away from home', 'I worry about others not liking me' and 'I get headaches or stomach aches when I am at school'. The items are scored on a 3-point scale, ranging from 'hardly ever', 'sometimes' to 'often'. In the current study, Cronbach's Alphas for early to middle and middle to late adolescents ranged from .92 to .95 across waves. 
Depression. Depressive symptoms were measured with the Children's Depression Inventory (CDI; Kovacs, 1985), a self-report questionnaire aimed at screening (subclinical) depressive symptomatology in children and adolescents. This scale has demonstrated convergent and discriminant validity, good internal consistency and adequate test-retest reliability in previous studies (Craighead, Smucker, Craighead, \& Ilardi, 1998; Hodges, 1990). The CDI consists of 27 items (e.g., 'I'm sad all the time'). The items were scored on a 3-point scale, ranging from 1 (false), to 3 (very true). Reliability of the CDI was high across waves for both early to middle and middle to late adolescents as Cronbach's Alphas ranged from .88 to .94 .

Delinquency. Delinquency was measured with an adapted version of a self-report questionnaire, measuring the frequency of several minor offences (Baerveldt, van Rossem, \& Vermande, 2003). The use of self-report data is widespread in criminology, and it is a valid instrument when restricted to petty crime (Baerveldt, 2000). Adolescents were asked how many times they had committed 14 minor offences, such as 'being caught by the police for doing something bad' or 'stealing a bike', in the past 12 months. The corresponding 14 items were scored on a 4-point scale, ranging from 1 (never), to 4 (four times or more). The original scale by Baerveldt et al. (2003) also contained items measuring drug use. These items were excluded from the delinquency scale. The slightly modified delinquency scale was reliable across waves in our sample: Reliability was acceptable among early to middle adolescents and middle to late adolescents, as Cronbach's alphas ranged from .77 to .92 .

Conflict. The Interpersonal Conflict Questionnaire (Laursen, 1993, 1995) was used to measure the frequency of conflicts. Adolescents indicated whether they had had an argument or fight with their fathers and mothers over the past 7 days with regard to 35 issues. A fivepoint Likert scale was used, ranging from never to often. Sample items are "manners," "privacy," "not doing what you are asked to do," "homework," and "being honest." The last item included the option "other," which adolescents could use to fill out an additional conflict topic not covered by the list. Similar to previous studies (e.g., van Doorn, Branje, \& Meeus, 2008), we averaged the 35 items to compute mean scores for conflict with fathers and mothers. Reliability was high for early to middle and middle to late adolescents, with Cronbach's alphas ranging from .94 to .96 across waves.

\section{Strategy of Analyses}

The first step in the current study was to calculate within-time normativeness, hypermaturity, and immaturity indices, using $q$-correlations. $Q$-correlations have a theoretical 
range from -1 to 1 , with higher figures indicating higher levels of within-time normativeness, hypermaturity, and immaturity. As such, $q$-correlations can be used as normal continuous variables, and can, therefore, be entered into correlation and regression analyses (e.g., Ozer \& Gjerde, 1989; Roberts, Caspi, \& Moffitt, 2001). Previous studies (e.g., Klimstra et al., 2009) found remarkable gender differences on Big Five personality dimensions. Therefore, we calculated within-time normativeness, hypermaturity, and immaturity indices for boys and girls separately.

To calculate within-time normativeness for 12-year-old boys, we first determined the mean scores of the 12-year-olds (i.e., T1 scores of early to middle adolescent boys) on the Big Five dimensions. Next, we calculated $q$-correlations between the resulting profile of the average 12-year-old boy (i.e., the profile of the average early to middle adolescent boy at T1) and the personality profiles of each individual 12-year-old boy. This procedure resulted in 468 $q$-correlations reflecting within-time normativeness for 12-year-old boys (range $=-.87,1.00$ ). In a similar way, we calculated within-time normativeness for 20 -year-old boys $(\mathrm{N}=169$; range $=-.58, .99), 12$-year-old girls $(\mathrm{N}=455$; range $=-.91, .99)$, and 20-year-old girls $(\mathrm{N}=$ 221 ; range $=-.58, .96)$.

To calculate hypermaturity for boys, we first calculated mean scores of 20 -year-old boys (i.e., T5 scores of middle to late adolescent boys) on all Big Five dimensions. In the next step, we calculated $q$-correlations between the resulting personality profile of the average 20 year-old boy (i.e., the profile of the average middle to late adolescent boys at T5) with personality profiles of each individual 12-year-old adolescent boy (i.e., early to middle adolescent boys at T1). The $q$-correlation then reflected the degree to which the personality profile of any 12-year-old adolescent boy in our sample matched the personality profile of an average 20-year-old boy, and hence reflected the degree of hypermaturity of 12-year-old adolescent boys. This procedure resulted in $468 q$-correlations representing hypermaturity for 12-year-old boys (range $=-.79$ to .99 ). With a similar procedure, we calculated $q$-correlations representing hypermaturity for four-hundred-and-fifty-five 12-year-old girls. These $q$ correlations ranged from -.81 to 1.00 .

Immaturity for boys was calculated in a similar way. First, mean scores of 12-year-old boys (i.e., T1 scores of early to middle adolescent boys) were calculated for each Big Five dimension, resulting in an average profile of a 12 -year-old boy. In the next step, $q$-correlations between the personality profile of an average 12-year-old boy (i.e., the profile of the average early to middle adolescent boy at T1) and each 20-year-old individual boy's (i.e., middle to late adolescent boys at T5) personality profile were assessed. This procedure resulted in 169 
$q$-correlations (ranging from -.82 to 1.00 ) reflecting the degree to which the personality profile of a 20 -year-old boy matched the profile of an average 12-year-old boy, and therefore indicated the degree of immaturity of a 20 -year-old individual boy. A similar procedure was followed to calculate $q$-correlations representing immaturity (range $=-.55$ to .97 ) for the twohundred-and-twenty-one 20-year-old girls in our sample.

As previously mentioned, $q$-correlations can be used as continuous variables and can therefore be entered into correlation and regression analyses. As such, we assessed partial correlations between hypermaturity and problem behavior, and immaturity and problem behavior as a second step of our strategy of analyses. In these analyses, we controlled for the associations between within-time normativeness and problem behavior symptoms.

\subsection{Results}

In order to conclude that personality development is off-time when a 12-year-old displays a personality profile similar to the profile of an average 20-year-old, or the 20-yearold displays a personality profile similar to the profile of an average 12-year-old, one should first examine whether there are dissimilarities between the profile of an average 12-year-old and an average 20-year-old. This turned out to be the case, as the profile similarity between the profile of an average 12-year-old boy and an average 20-year-old boy was .78. For girls, the $q$-correlation representing similarity between these two profiles was .64. Because these figures were well below 1.00, we could proceed to the second step in our examination of the implications of having a hypermature or immature personality profile: the calculation of indices for hypermaturity and immaturity with $q$-correlations. Descriptive statistics for Big Five personality traits (on which our indices of within-time normativeness, hypermaturity, and immaturity are based), within-time normativeness, hypermaturity and immaturity indices, and problem behavior are displayed in Table 6.1, for boys and girls separately.

In the next step, we assessed how hypermaturity and immaturity were associated with problem behavior using partial correlations. To make sure we dealt with the unique effects of our hypermaturity and immaturity indices, we controlled for the effects of within-time normativeness. The correlations of hypermaturity and immaturity with problem behavior in which the effects of within-time normativeness were controlled for, are depicted in Table 6.2. 
Table 6.1

Means and Standard Deviations of Boys and Girls for Big Five Personality Traits, Hypermaturity, Immaturity and Problem Behavior

\begin{tabular}{|c|c|c|c|c|}
\hline & \multicolumn{2}{|c|}{ Boys } & \multicolumn{2}{|c|}{ Girls } \\
\hline & Age 12 & Age 20 & Age 12 & Age 20 \\
\hline & $(N=468)$ & $(N=169)$ & $(N=455)$ & $(N=221)$ \\
\hline & $M(S D)$ & $M(S D)$ & $M(S D)$ & $M(S D)$ \\
\hline \multicolumn{5}{|l|}{ Big Five traits } \\
\hline Extraversion & $4.87(.98)$ & $4.93(1.13)$ & $4.95(1.05)$ & $4.77(1.18)$ \\
\hline Agreeableness & $4.93(1.16)$ & $5.68 \quad(.60)$ & $5.20 \quad(.97)$ & $5.73(.55)$ \\
\hline Conscientiousness & $4.05(1.13)$ & $4.32(1.18)$ & $4.23(1.09)$ & $4.71(1.19)$ \\
\hline Emotional Stability & $4.70(1.16)$ & $4.77 \quad(.99)$ & $4.57(1.08)$ & $4.24(1.04)$ \\
\hline Openness & $4.38(1.14)$ & $4.95(.85)$ & $4.40(1.01)$ & $4.87(.86)$ \\
\hline Normativeness & $.45 \quad(.46)$ & $.55 \quad(.33)$ & $.43(.45)$ & $.54(.28)$ \\
\hline Hypermaturity & $.40(.40)$ & -- & $.43(.38)$ & -- \\
\hline Immaturity & -- & $.43(.47)$ & -- & $.38(.38)$ \\
\hline \multicolumn{5}{|l|}{ Problem behavior } \\
\hline Anxiety & $1.30(.31)$ & $1.19(.20)$ & $1.33(.26)$ & $1.32(.26)$ \\
\hline Depression & $1.16(.29)$ & $1.11(.15)$ & $1.16(.21)$ & $1.19(.22)$ \\
\hline Delinquency & $1.28(.49)$ & $1.14(.25)$ & $1.11(.32)$ & $1.04(.08)$ \\
\hline Conflict & $1.77(.50)$ & $1.44(.41)$ & $1.64(.46)$ & $1.29(.35)$ \\
\hline
\end{tabular}

Note. Hypermaturity represents a $q$-correlation of actual personality profiles of 12 -year olds with the average personality profile of a 20 -year old individual. Immaturity reflects a $q$-correlation of actual personality profiles of 20-year olds with the average personality profile. Normativeness is Within-Time Normativeness.

Table 6.2 reveals that hypermaturity in early adolescent boys and girls was associated with internalizing problem behavior, as hypermaturity was positively correlated with levels of anxiety and depression. For girls, higher levels of hypermaturity were additionally associated with higher levels of conflict. Correlations with externalizing problem behavior did not reach significance.

Higher levels of immaturity were related to lower levels of anxiety in boys and girls. In girls, immaturity was also negatively associated with levels of anxiety, but it was positively associated with levels of delinquency. 
Table 6.2

Partial Correlations (Controlled for Within-Time Normativeness) between Hypermaturity and Immaturity, and Problem Behavior for Adolescent Boys and Girls

\begin{tabular}{|c|c|c|c|c|c|c|c|c|}
\hline \multirow[b]{2}{*}{ Boys } & \multicolumn{2}{|c|}{ Anxiety } & \multicolumn{2}{|c|}{ Depression } & \multicolumn{2}{|c|}{ Delinquency } & \multicolumn{2}{|c|}{ Conflict } \\
\hline & & & & & & & & \\
\hline Hypermaturity $(N=468)$ & $.16^{* *}$ & $(-.03)$ & $.10^{*}$ & $(-.02)$ & -.04 & $(-.06)$ & .03 & $(.01)$ \\
\hline Immaturity $(N=169)$ & $-.29 * * *$ & $(-.26 * * *)$ & $-.16^{*}$ & $(-.13)$ & .12 & $(.09)$ & -.04 & $(.01)$ \\
\hline \multicolumn{9}{|l|}{ Girls } \\
\hline Hypermaturity $(N=455)$ & $.34 * * *$ & $(.23 * * *)$ & $.35 * * *$ & $\left(.26^{* * *}\right)$ & -.07 & $(-.06)$ & $.14 * *$ & $(.14 * *)$ \\
\hline Immaturity $(N=221)$ & $-.33 * * *$ & $(-.25 * * *)$ & -.12 & $(-.06)$ & $.22 * *$ & $(.22 * *)$ & -.02 & $(.01)$ \\
\hline
\end{tabular}

Note. ${ }^{*} p<.05 ; * * p<.01 ; * * * p<.001$. Zero-order correlations are presented between brackets.

\subsection{Discussion}

In the current study, we explored the correlates of two types of cross-time personality profile normativeness: hypermaturity and immaturity. Overall, our findings suggest that girls were more affected by off-time personality development than boys, and that hypermaturity was positively associated with internalizing problems and conflict, whereas immaturity was related to externalizing problems.

Girls who reflected high levels of developmental hypermaturity (i.e., displaying a personality profile resembling the profile of an average a 20 -year-old at age 12) exhibited several problems. They were more anxious and depressed, and reported more conflicts with their parents. Hypermaturity in boys was also positively associated with internalizing problems (i.e., anxiety and depression), but, unlike girls, boys did not exhibit higher levels of conflict with parents when they displayed hypermaturity of personality. Interestingly, similar findings have been obtained in studies regarding early pubertal timing (for an overview, see Graber, 2003). These studies suggest that facing the physical transitions accompanying pubertal development (i.e., rapid growth spurt, menarche) well before one's peers do may results in psychosocial problems, because early maturing adolescents may simply be less prepared for the consequences of obtaining and adult-like posture (e.g., Brooks-Gunn, Petersen, \& Eichorn, 1985). Thus, they may elicit responses from others for which they are 
not developmentally ready. On the contrary, individuals with a hypermature personality profile may elicit responses from significant others that they may perceive as childish. If individuals feel like they are being treated in an age-inappropriate manner, they may experience a poor stage-environment fit which could consequently lead to negative outcomes, such as lower levels of self-esteem (Eccles et al., 1993). This mechanism could possibly also explain the higher levels of internalizing problems among adolescent with a hypermature personality in the current study.

Hypermature girls, but not hypermature boys, also experience elevated levels of conflict with parents. It has been shown that connectedness plays a central role in psychological development of adolescent girls, but to a much lesser extent in adolescent boys (e.g., Geuzaine, Debry, \& Liesens, 2000). In this context, boys have for example been shown to keep more secrets from parents when compared to girls (Keijsers, Branje, Frijns, Finkenauer, \& Meeus, 2010). When compared to boys, hypermature girls may therefore be more likely to experience a poor stage-environment in the relationship with their parents, which could lead to discontent with this relationship. An obvious way to exhibit this discontent is to engage in more conflicts with parents.

Immaturity of personality was associated with lower levels of anxiety in both boys and girls. Thus, immaturity of personality among both boys and girls seems to be reflected in some sort of fearlessness. Childhood fearlessness has been shown to be predictive of externalizing problems in adolescence (Shaw, Gilliom, Ingoldsby, \& Nagin, 2003). Findings of the current study are to some extent similar, as girls with an immature personality profile reflected both lower levels of anxiety and higher levels of externalizing problems (i.e., delinquency). However, low levels of anxiety in immature boys were not accompanied by higher levels of externalizing problems, but with lowered levels of depressive symptoms. This would suggest that the low levels of anxiety that accompanied immaturity of personality reflect plain boldness in boys, but have a different meaning in girls. A first possible explanation for immature girls reflecting high levels of risk behaviour can be derived from studies on general personality development. Klimstra et al. (2009) showed that girls tend to mature earlier with regard to personality than boys. The girls that do lag behind with regard to personality development might simply be more like boys, and hence display similar patterns of risk behaviours than boys. In the current study (see Table 1), and in general (e.g., Arnett, 1999), boys tend to display higher levels of delinquency. This could explain why immature girls, who may be more similar to boys then their less immature counterparts, also display higher levels of delinquency. An alternative explanation could be related to gender differences 
in social expectations. Immaturity of personality might be much more social acceptable for boys than for girls as men typically take up adult roles (e.g., marriage, starting a family) at a later age than women do. In fact, Dutch national demographic data showed that the average woman is more than 3 years younger then the average man when she gets married and gets children (Statistic Netherlands, 2009a, 2009b). Thus, boys may not be expected to exhibit a mature personality, whereas girls are. As a result, immature girls may fail to meet social expectations, whereas this is not necessarily the case for immature boys. Not meeting social expectations has been described as possible cause of losing social approval (Roberts et al., 2006). Engagement in antisocial activities, such as delinquency, might reflect a reaction to this withdrawal of social approval.

\section{Limitations}

Although the present study provides an interesting perspective on off-time personality development, several limitations should be recognized. First of all, our indices of hypermaturity and immaturity only provide a glance at what the consequences of being ahead or lagging behind normative developmental trends in personality could be. In other words, conceptualizing hypermaturity as younger individuals having a personality profile resembling the typical profile of an older individual, and immaturity as older individuals having a personality profile resembling the typical profile of a younger individual, only provides a rough estimate of what the consequences of off-time personality development could be. We did correct for the effects that within-time normativeness (i.e., the similarity between an individual's personality profile and the profile of their peers) had on problem behavior symptoms, in order to make sure that we did measure the unique effects of our measures of hypermaturity and immaturity. The fact that effects of hypermaturity and immaturity were still present after correcting for the effects of within-time normativeness underscores the potential importance of these two concepts, but future research could still focus on more refined measures of developmental hypermaturity and immaturity of personality.

A second limitation concerns the number of measurement occasions of hypermaturity and immaturity. Personality traits do change during adolescence (e.g., Klimstra et al., 2009), but a large interval between measurement occasions is needed to obtain sufficiently different personality profiles to calculate hypermaturity and immaturity. Between ages 12 and 16, there is, for example, very little change in boys' personality traits, which implies that the personality profile of an average 12-year-old boy is almost identical to the average personality profile of a 16-year old boy. In such cases, a 16-year-old boy reflecting a profile strongly 
Hypermaturity and Immaturity

resembling the profile of an average 12-year-old boy can not be considered immature. Nevertheless, future studies should try to get multiple measurement occasions of hypermaturity and immaturity in order to investigate stability and change in these two facets of personality, and to examine what variables are predictive of or are predicted by hypermaturity and immaturity.

Third, we used data from two cohorts to calculate hypermaturity and immaturity indices. That is, we correlated the profile of an average early to middle adolescent at T1 with profiles of individual middle to late adolescents at T5 to calculate immaturity, and correlated profiles of individual early to middle adolescents with the profile of an average middle to late adolescent at T5. As such, cohort effects (see Twenge, 2000, 2001, for a discussion of cohort effects) might be somewhat confounded with hypermaturity and immaturity indices. Ideally, hypermaturity and immaturity indices should be calculated using from one single longitudinally examined cohort.

A fourth potential limitation concerns the use of adolescent self-reports. The use of self-reports implies that our findings reflect correlates of hypermaturity and immaturity of an adolescents self-perceived personality profile. Although the psychometrics of self-reports of adolescent personality have been shown to be accurate (Soto, John, Gosling, \& Potter, 2008), it would still be interesting to examine if hypermaturity and immaturity indices based on other-reported personality data yield the same conclusions.

\section{Conclusion}

Despite these potential limitations, the present study provides a first glance into what the correlates of hypermaturity and immaturity of personality in adolescent boys and girls could be. Our analyses revealed that hypermature boys and girls reflect internalizing problems, whereas hypermature girls additionally exhibited high levels of conflict with parents. Both immature boys and girls reflected lower levels of anxiety than their less immature counterparts. For girls, immaturity was additionally associated with high levels of externalizing problems (i.e. delinquency). These findings underscore the potential importance of examining causes and consequences of off-time personality development, and the role of gender in these processes. 


\section{Chapter 7}

Identity Formation in Adolescence: Change or Stability? ${ }^{7}$

${ }^{7}$ Klimstra, T. A., Hale, W. W., Raaijmakers, Q. A. W., Branje, S. J. T., \& Meeus, W. H. J. (2010). Identity formation in adolescence: Change or stability? Journal of Youth and Adolescence, 39, 150-162. 


\begin{abstract}
The aim of this five-wave longitudinal study of 923 early to middle adolescents ( $50.7 \%$ boys; $49.3 \%$ girls) and 390 middle to late adolescents ( $43.3 \%$ boys and $56.7 \%$ girls) is to provide a comprehensive view on change and stability in identity formation from ages 12 to 20. Several types of change and stability (i.e., mean-level change, rank-order stability, and profile similarity) were assessed for three dimensions of identity formation (i.e., commitment, in-depth exploration, and reconsideration), using adolescent self-report questionnaires.

Results revealed changes in identity dimensions towards maturity, indicated by a decreasing tendency for reconsideration, increasingly more in-depth exploration, and increasingly more stable identity dimension profiles. Mean levels of commitment remained stable, and rankorder stability of commitment, in-depth exploration, and reconsideration did not change with age. Overall, girls were more mature with regard to identity formation in early adolescence, but boys had caught up with them by late adolescence. Taken together, our findings indicate that adolescent identity formation is guided by progressive changes in the way adolescents deal with commitments, rather than by changes in the commitments themselves.
\end{abstract}




\subsection{Introduction}

Erikson proposed that identity formation is the key developmental task of adolescence (Erikson, 1972). Therefore, identity formation has been hypothesized to involve "changes in identity that can be characterized as progressive developmental shifts" (Waterman, 1982; $\mathrm{p}$. 355 , italics added). However, there is a lack of longitudinal studies investigating the dynamics of identity formation across the entire period of adolescence. Moreover, there has been a debate on whether identity formation is better characterized by change or by stability (Van Hoof, 1999; Waterman, 1999). The current study aims to provide a comprehensive view on adolescent identity formation by examining various aspect of change and stability, using fiveannual-wave longitudinal data on early to middle and middle to late adolescent boys and girls.

The most commonly used conceptualization of Erikson's identity theory is Marcia's identity status paradigm (Marcia, 1966). In this paradigm, the focus is on two dimensions central to Erikson's work on identity: exploration of developmental alternatives in various salient identity-defining domains (referred to as "crisis" in Erikson's work); and selection of alternatives as well as engaging in relevant activities towards the implementation of these choices (commitment). Marcia proposed that, based on the amounts of exploration and commitment, an adolescent's identity can be classified into either one of four distinguishable identity statuses: diffusion (low in exploration and low on commitment), foreclosure (little exploration, but strong commitments), moratorium (high on exploration, but no stable commitments as yet), and achievement (high on commitment after a period of extensive exploration). There is consensus that diffusion should be considered as the least adaptive status, foreclosure and moratorium are two intermediate statuses, and achievement is the most adaptive status (Waterman, 1999).

\section{Studies on Identity Formation: From a Status Approach towards a Dimensional Approach}

Marcia's identity status paradigm (Marcia, 1966), originally developed as an attempt to classify an adolescent's identity status at a certain point in time (Marcia, 2007), has inspired hundreds of empirical investigations of identity formation (for reviews, see for example: Meeus, 1996; Kroger, 2007). Following Erikson's assumption that identity formation involves a developmental process (Erikson, 1972, 1974), a limited number of these studies has used a longitudinal approach. These studies have assessed changes in identity formation in either one of two ways: (1) by focusing on changes in identity status, or (2) by focusing on changes in separate identity dimensions. We will first discuss studies on changes in identity 
status, and then discuss the merits of studying longitudinal changes in identity formation with separate measures of commitment and exploration.

In a review of studies examining identity status change, Waterman $(1982,1999)$ concluded that the basic hypothesis underlying Erikson's work on identity ("movement from adolescence to adulthood involves changes in identity that can be characterized as progressive developmental shifts", p. 355, italics added), has received support in empirical studies. This hypothesis is also referred to as the fundamental developmental hypothesis of identity formation. The progressive developmental shifts Waterman (1982) refers to are changes from less adaptive identity statuses (e.g., diffusion) towards the most adaptive status (i.e., achievement). However, not everyone agrees with Waterman's notion of progressive developmental shifts. Based on the same studies where Waterman (1999) noted progressive changes, Van Hoof (1999) concluded that identity status studies have more often found stability than change. Yet, she did note that if change does occur it is more likely to be progressive than regressive. The contradiction between the conclusions of Waterman and Van Hoof is caused by the fact that Waterman emphasized that progressive change outweighs regressive change, whereas Van Hoof stressed that stability occurred more often than progressive changes did. Both positions received support in a recent meta-analysis by Kroger (2007), as she found equal probabilities of stability and progressive developmental changes in identity formation, while progressive change was much more common than regressive change. In sum, previous studies on identity status change indicate that identity formation in adolescence is either characterized by stability, or by progressive change. Apart from a debate concerning the amount of change in identity formation, there is also disagreement on the timing of changes in identity formation in adolescence. Several overview studies (e.g., Marcia, 1980; Waterman, 1982, 1993) concluded that changes in identity formation were most likely to occur in late adolescence, whereas Meeus, Iedema, Helsen \& Vollebergh (1999) found that changes were just as common in early and middle adolescence, as in late adolescence.

Thus, longitudinal studies on changes in identity status have not led to consensus on either the direction or the timing of changes with regard to identity formation. This could be caused by the fact that studies on identity status change merely provide a rough estimate of the overall direction of identity formation and are not informative on longitudinal changes in the underlying dimensions of commitment and exploration (Matteson, 1977). Identity status changes only occur when the amount of change in the underlying dimensions passes a certain threshold, whereas an approach with a focus on separate dimensions of commitment and 
exploration is also sensitive to smaller changes in identity formation (Meeus, 1996). For that reason, studies focusing on separate identity dimensions could shed new light on the change versus stability debate (van Hoof, 1999; Waterman, 1999) that has mainly been related to the identity statuses until now. Therefore, the current study aims to contribute to the change versus stability debate by examining the developmental course of the dimensions underlying the statuses.

Several identity models with a focus on separate dimensions of exploration and commitment have been introduced since the late 1990s (Balistreri, Busch-Rossnagel, \& Geisinger, 1995; Luyckx, Goossens, Soenens, \& Beyers, 2006; Meeus, 1996). In the current study, a recently developed three-dimension model will be applied to assess stability and change in identity formation. We will now discuss this three-dimension model, and then review the few longitudinal studies that have followed a dimensional approach to identity formation.

\section{A Three-dimension Model of Identity Formation}

In 2001, Meeus designed a new measure to assess identity formation: the UtrechtManagement of Identity Commitments Scale (U-MICS; Crocetti, Rubini, \& Meeus, 2008). UMICS is partly based on a previous measure, the Utrecht-Groningen Identity Development Scale (U-GIDS; Meeus, 1996), as it contains commitment and in-depth exploration scales. However, a relatively new exploration scale was added: reconsideration.

The definition of commitment in U-MICS (Crocetti, Rubini, \& Meeus, 2008) is similar to the original definition by Marcia (1966), and refers to being committed to one's choice of identity. However, whereas Marcia assessed one type of exploration, two types of exploration are distinguished in U-MICS: in-depth exploration and reconsideration of commitment. Indepth exploration represents the extent to which adolescents explore current commitments actively, reflect on their choices, search for additional information about these choices, and discuss their current commitments with relevant others (i.e., friends or family). It refers to adolescents exploring the merits of their current commitments, without questioning the commitments themselves. Reconsideration of commitment refers to adolescents comparing their present commitments with possible alternative commitments. When reconsideration occurs, it has a short-term detrimental effect on adolescents, as it reflects uncertainty about commitments and is therefore positively related to problem behaviors such as depression and delinquency (Crocetti, Rubini, \& Meeus, 2008). However, when the developmental context of an individual changes, it can be necessary to re-evaluate or even replace old commitments. 
Thus, the long-term effects may, in fact, be positive since adapting one's commitments can be necessary when current commitments are no longer suitable in a new context.

The three dimensions of U-MICS cover three important aspects from the broader Eriksonian perspective on identity formation and Marcia's elaboration on that perspective. First, reconsideration involves comparisons of present commitments with possible alternatives, and the possible replacement of present commitments as a result of these comparisons. Thereby, reconsideration captures an important facet of identity formation as described by Erikson (1972), who stated that "in puberty and adolescence all samenesses and continuities relied on earlier are more or less questioned again" (pp. 252-253). Second, the three-fold conceptualisation of identity formation involves a double evaluation process of present commitments, comparable to a recent addition to Marcia's identity status paradigm: the dual-cycle model proposed by Luyckx, Goossens, and Soenens (2006). They distinguish between a commitment formation cycle, referring to the formation of stable commitments, and a commitment evaluation cycle, capturing the process of evaluating and actively thinking about commitments. Since reconsideration refers to comparing present commitments with alternative ones, decreases in reconsideration signify increases in certainty about current commitments. Therefore, reconsideration partly fits into the commitment formation cycle. Indepth exploration fits into the commitment evaluation cycle because it indicates an adolescent's reflection on his/her present commitments, without questioning the commitments themselves.

Third, although Meeus and colleagues (Crocetti, Rubini, \& Meeus, 2008) distinguish two instead of one exploration dimension (e.g., in-depth exploration and reconsideration), they were able to replicate the four identity statuses Marcia (1966) originally found (Crocetti, Rubini, Luyckx, \& Meeus, 2008). However, while Marcia only found one moratorium status, Crocetti et al. (2008) found two distinctive moratoria-statuses (i.e., moratorium and searching moratorium). Even so, the findings of Crocetti et al. (2008) demonstrate the convergent validity of the three-dimension model.

In this study, the three U-MICS dimensions (Crocetti, Rubini, \& Meeus, 2008) will be used to assess identity formation through adolescence. Similar to other identity measures like the Identity Status Interview (ISI; Marcia, 1966), the Extended version of the Objective Measure of Ego Identity Status (EOM-EIS; e.g., Bennion \& Adams, 1986), and the Ego Identity Process Questionnaire (EIPQ; Balistreri et al., 1995), U-MICS allows identity dimensions to be assessed in the ideological domain (i.e., education) and the interpersonal domain (i.e., friendships). However, as the main aim of the current study is to examine change 
and stability in identity formation in general and not to assess differences between identity domains, identity dimensions will be assessed at a global level. Crocetti, Rubini, and Meeus (2008) showed that U-MICS is well-suited for measuring global identity dimensions, as they have demonstrated that collapsing ideological and interpersonal dimensions into global identity dimensions did not affect the factor structure or the reliability of U-MICS.

\section{Three Types of Change in Identity Dimensions}

Change can be operationalized in several ways. In the related field of adolescent personality development, a case has been made for the assessment of various distinct types of change in one research design to obtain a comprehensive perspective on development. These types of change are: changes in mean levels, changes in the rank-order of individuals, and changes in profiles consisting of various traits (e.g., Block \& Robins, 1993). All three components add a unique piece of information, and together they provide a comprehensive perspective on development. Several studies in the field of personality have followed such an approach (e.g., De Fruyt et al., 2006; Roberts, Caspi, \& Moffitt, 2001; Robins, Fraley, Roberts, \& Trzesniewski, 2001), yet all three change components have not been used together in one research design for the examination of identity formation. Two components have been examined in previous studies on change and stability in identity commitment and exploration: mean-level change and rank-order stability. The third, profile similarity, is new to the field of identity formation. We will now discuss these three indices of change and stability, and explain how they complement one another.

\section{Mean-level Change}

The most obvious way to examine progressive changes in identity dimensions is to assess whether mean scores of a population on commitment and exploration change in a favourable direction. Only three longitudinal studies have followed such an approach. In these studies, Meeus et al. (1999) found increases of commitment and in-depth exploration during adolescence. Luyckx, Goossens, and Soenens (2006) and Luyckx, Schwartz, Goossens, Soenens, and Beyers (2008) also found an increase in in-depth exploration. Luyckx, Goossens, and Soenens (2006) further demonstrated increases in exploration of alternative commitments (they label this dimension as exploration in breadth, but it is conceptually quite similar to the reconsideration dimension assessed with U-MICS), but Luyckx et al. (2008) showed that this increase was followed by a decrease. Luyckx and colleagues distinguish two commitment dimensions: commitment making and identification with commitment. For 
identification with commitment, the dimension that is most similar to the commitment dimensions by Marcia (1966) and Crocetti, Rubini, and Meeus (2008), decreases over time were found. Thus, there is agreement on longitudinal increases for in-depth exploration, but findings concerning commitment are inconsistent and call for more longitudinal research. Findings for exploration in breadth (i.e., conceptualized as reconsideration in the present study) suggest that increases are followed by decreases. This is in line with Marcia's (1966) reasoning that the most advanced identity status (i.e., achievement) represents strong commitments formed after a period of exploration in breadth, and suggests that adolescents indeed seem to move towards an achieved identity as they grow older (e.g., Waterman, 1982, 1999). Based on the above mentioned studies, we expect adolescent identity formation in the three-dimension model used in the current study (Crocetti, Rubini, \& Meeus, 2008) to be characterized by increases for in-depth exploration and a decreasing tendency to reconsider commitment. Furthermore, this study might clear up the inconsistent pattern of development of commitment found in previous studies.

The aforementioned longitudinal studies did not add to the earlier mentioned debate on the timing of identity formation in adolescence, as they did not compare the amount of change in identity dimensions in early to middle and middle to late adolescence. The design of the current study allows for a longitudinal exploration of where in adolescence changes are most common.

\section{Rank-order Stability}

Indices of mean-level change are only informative on the average direction and amount of change in a sample. Rank-order stability, on the other hand, indicates whether the rank-order of individuals on a certain trait is maintained over time. Mean-levels of an identity dimension could change, but these changes only represent normative development reflecting universal maturation processes if they are accompanied by high levels of rank-order stability (e.g., Roberts \& DelVecchio, 2000). Thus, indices of rank-order stability are needed alongside measures of mean-level change to indicate whether the observed changes apply to a majority of individuals in a sample. In addition, changes in rank-order stability itself can indicate when in adolescence inter-individual differences in several dimensions of identity formation start to become more settled.

There are three studies examining rank-order stability of identity dimensions. Over a six-month period, Luyckx et al. (2008) found relatively high rank-order stability for identity dimensions and both Luyckx, Goossens, and Soenens (2006) and Luyckx, Soenens et al. 
(2006) reported similar results over a one-year and a two-year period (correlations ranging from .40 - .77). All studies indicated that there was a substantial amount of within-group stability. Since previous studies have shown that rank-order stability of identity dimensions is high over a period up to two years, we expect at least medium rank-order stability (i.e., higher than .30; Cohen, 1988) for identity dimensions over a four-year period.

To determine in what part of adolescence inter-individual differences in change are most common, and thus to investigate when inter-individual differences in identity dimensions start to become more settled, indices of rank-order stability in various periods of adolescence have to be compared. The current study is the first that will explore differences in rank-order stability in early, middle and late adolescence.

\section{Profile Similarity}

A third aspect of change and stability, profile similarity, has so far not been assessed in the field of identity formation. The technique has been applied in the related field of adolescent personality research (e.g., Block, 1971; Ozer \& Gjerde, 1989; Roberts et al., 2001). Profile similarity provides information on the stability of a constellation of traits over time within a person, and is therefore, unlike rank-order stability and mean-level change, not informative on changes in individual identity dimensions. Because profile similarity indicates to what extend a person's configuration of identity dimensions changes over time, it is also different from other change indices and is not indicative of changes at the sample level. Profile similarity is usually calculated with $q$-correlations. Like Pearson correlations, $q$ correlations range from -1 to 1 . The higher the $q$-correlation, the more stable a constellation of identity dimensions within a person is (e.g., Ozer \& Gjerde, 1989; Roberts et al., 2001). In the current study, profile similarity is defined as intra-individual change in the rank-ordering of three identity dimensions (commitment, in-depth exploration, and reconsideration). High levels of profile similarity would indicate that an adolescent has a steady identity profile, whereas low levels of profile similarity would indicate profound fluctuations in the shape of an identity profile. Thus, the amount of profile similarity is to some extent informative on the stability of identity structures of adolescents.

As profile similarity has, so far, not been used in the field of identity formation research, our examination of this change index will be exploratory in nature. This examination may provide additional information on whether identity formation mainly takes place in late adolescence, as advocated by Marcia (1980) and Waterman (1982, 1993), or throughout the entire adolescent period, as Meeus (1996) presumes. 


\section{Gender Differences}

Debates relating to gender differences in identity formation can be traced back to Erikson (1974) who claimed that women would have to keep their identities open, to adjust to the peculiarities of the men they would eventually meet and to the children they would raise. Nowadays, such a statement should be considered as outdated in Western countries, since both men and women pursue occupational careers and childrearing has become much more of a mutual process with men and women sharing such tasks. Considering these societal changes, gender differences in identity formation might have disappeared as well. In fact, several researchers (e.g., Kroger, 1997; Waterman, 1982, 1993, 1999) concluded in overviews that there were no general differences in the prevalence of identity statuses among men and women, a conclusion that has been reconfirmed in an extensive empirical study by Meeus et al. (1999). Notwithstanding a lack of evidence for gender differences in the prevalence of identity statuses, it is still possible that there are gender differences in the identity dimensions underlying the statuses (i.e., exploration and commitment). Recent longitudinal studies on identity dimensions have mainly been conducted by Luyckx and colleagues (Luyckx, Goossens, \& Soenens, 2006; Luyckx et al., 2008). They employed samples predominantly comprised of females, and were therefore unable to test for gender differences. Thus, gender differences in longitudinal changes and stability of identity dimensions of exploration and commitment yet need to be investigated. The current study will set out to explore such gender differences in all three mentioned aspects of change (i.e., mean-level change, rank-order stability, and profile similarity).

\section{Aims of the current study}

The aim of this study is to examine how adolescent identity formation is best described. We expect identity formation to be described by mean-level increases in in-depth exploration and a decreasing tendency for reconsideration. This study also seeks to clarify the inconsistent pattern of development of commitment found in previous studies. Two other research questions will be examined in an explorative manner. First, we will use indices of rank-order stability to determine where in adolescence inter-individual differences in three separate identity dimensions start to become more settled. Second, we will assess profile similarity of identity dimensions to find out where in adolescence identity profiles including three identity dimensions start to become more stable. In addition, gender differences in all three aspects of change and stability will be explored. 


\subsection{Method}

\section{Participants}

Data for this study were collected as part of a five-wave longitudinal research project on CONflict And Management Of RElationships (CONAMORE; Meeus et al., 2006), with a one-year interval between each of the waves. The longitudinal sample consisted of 1,313 participants divided into an early to middle adolescent cohort $(n=923 ; 70.3 \%)$, who were 12.4 years of age on average $(S D=.59)$, and a middle to late adolescent cohort $(n=390$; $29.7 \%)$ with an average age of 16.7 years $(S D=.80)$ during the first wave of measurement. The early to middle adolescent cohort consisted of 468 boys (50.7\%) and 455 girls (49.3\%), the middle to late adolescent cohort consisted of 169 boys (43.3\%) and 221 girls (56.7\%). Because both age groups were assessed during five measurement waves, a total age range from 12 to 20 years was available. Participating adolescents were recruited from various randomly selected junior high and high schools in the province of Utrecht, The Netherlands. As the composition of the population of Utrecht closely mirrors that of the Dutch population as a whole (Statistics Netherlands, 2003), our sample was representative for the general Dutch adolescent population.

Sample attrition was $1.2 \%$ across waves: in waves $1,2,3,4$, and 5 the number of participants was 1,313,1,313,1,293, 1,292 and 1,275, respectively. Across waves $2.6 \%$ of the data was missing. Missing values were estimated in SPSS, using the EM-procedure. Little's Missing Completely At Random Test (Little, 1988) revealed a normed $\chi^{2}\left(\chi^{2} / \mathrm{df}\right)$ of 1.78 , which according to guidelines by Bollen (1989) indicates a good fit between sample scores with and without imputation.

\section{Procedure}

Participants and their parents received an invitation letter, describing the research project and goals, and explaining the possibility to decline from participation. More than $99 \%$ of the approached high school students decided to participate. All participants signed the informed consent form. The questionnaires were completed at the participants' own high school, during annual assessments. Confidentiality of responses was guaranteed. Verbal and written instructions were offered. The adolescents received $€ 10$ (approximately US \$15) as a reward for every wave they participated in. 


\section{Measures}

Identity Formation. Identity formation was assessed with the Utrecht-Management of Identity Commitments Scale (U-MICS), a self-report measure designed by Meeus (Crocetti, Rubini, \& Meeus, 2008) based on the U-GIDS (Meeus, 1996). With this instrument, 5-point Likert-scale items, with a response format ranging from 1 (completely untrue) to 5 (completely true), are used to assess three identity dimensions: commitment (five items), in-depth exploration (five items), and reconsideration (three items). The same items can be filled out to assess identity dimensions in different domains. In the current study, we focused on one ideological domain (i.e., education) and one interpersonal domain (i.e., friendships) that play an important role in the lives of all adolescents.

Because we assessed two domains with 13 items each, the total number of items of UMICS was 26 in the current study. Sample items are: "My education/best friend gives me certainty in life" (ideological/interpersonal commitment), "I think a lot about my education/best friend" (ideological/interpersonal in-depth exploration), "I often think it would be better to try and find different education/a different best friend" (ideological/interpersonal reconsideration). Although U-MICS allows for identity dimensions to be measured in different content domains, we focused on identity dimensions at a global level. For this purpose we followed the scale construction procedure described by Crocetti, Rubini, and Meeus (2008). A detailed description of the validity of U-MICS is provided by Crocetti, Rubini, and Meeus (2008), and Crocetti, Rubini, Luyckx, and Meeus (2008). Reliability of UMICS was high across waves for commitment (Cronbach's alphas .91 - .95), in-depth exploration (Cronbach's alphas .84 - .92), and reconsideration (Cronbach's alphas .92 - .94).

\subsection{Results}

\section{Mean-level Change in Identity Dimensions}

Mean-level change in identity dimensions was estimated with a multivariate Latent Growth Curve Model (LGCM; Duncan, Duncan, Stryker, Li, \& Alpert, 1999) in Mplus (Muthén \& Muthén, 1998-2007). LGCM provides mean levels (i.e., intercepts) and mean change rates (i.e., slopes), which are based on individual growth trajectories of all participants. Maximum Likely Robust estimation (MLR) was used, as MLR gives the most accurate estimate of chi-squares when the distribution of scores deviates from a normal distribution (Satorra \& Bentler, 1994), which turned out to be the case for scores on our identity formation measure. 
We used a multigroup longitudinal design with four groups: early to middle adolescent boys and girls, and middle to late adolescent boys and girls. Because there was a one-wave overlap between the early to middle and middle to late adolescent cohorts, we were able to infer an accelerated perspective (e.g., Duncan et al., 1999) by placing the intercept on the one wave of overlap between these two age cohorts (i.e., slope factor loadings were $-4,-3,-2,-1$, and 0 for the five consecutive measurement occasions for early to middle adolescent boys and girls, and $0,1,2,3$, and 4 for middle to late adolescent boys and girls). The accelerated design required us to constrain the means and variances of intercepts in the two cohorts to be equal. Chi-square difference tests revealed that an accelerated approach was justified, since the fit of a model with means and variances of intercepts constrained to be equal, did not significantly differ from the fit of a model where means and variances of intercepts were freely estimated. Means and variances of slopes were allowed to vary between the two cohorts, enabling us to examine whether developmental patterns of identity dimensions were different in early to middle and middle to late adolescence.

To determine what shape of growth characterized our data best, we first ran univariate accelerated Latent Growth Curve Models for each of the three identity dimensions (i.e., commitment, in-depth exploration, and reconsideration). Different types of latent growth models were estimated, that is: no growth, linear growth, and quadratic growth. The best model was chosen by comparing chi-squares of different models, using the procedure proposed by Satorro and Bentler (2001). Model fit was further judged by assessing RMSEA's, CFI's, and TLI's. RMSEA's smaller than .08, and CFI's and TLI's larger than 95 indicate an adequate model fit (Hu \& Bentler, 1999), and relatively lower RMSEA's, and higher CFI's and TLI's indicate better fits when comparing models (Kline, 1998). Our univariate models revealed that commitment and in-depth exploration were best characterized by linear growth, whereas reconsideration was best characterized by curvilinear growth.

Second, we ran a multivariate multigroup model including growth curves of commitment, in-depth exploration, and reconsideration. To improve model fit, intercepts and slopes were allowed to correlate within a certain identity dimension, as well as between dimensions. Observed means and standard deviations of commitment, in-depth exploration, and reconsideration for early to middle and middle to late adolescent boys and girls, are presented in Table 7.1 . 


\begin{tabular}{|c|c|c|c|c|c|}
\hline 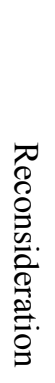 & 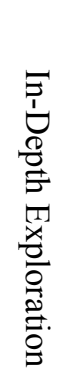 & 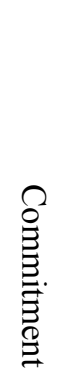 & 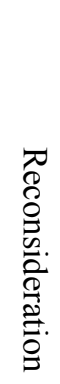 & 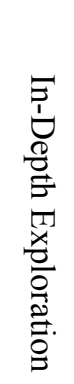 & 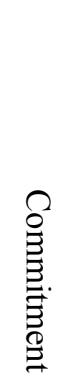 \\
\hline$\overbrace{\substack{\infty \\
\stackrel{\infty}{\omega}}}$ & $\begin{array}{l}\stackrel{\omega}{\tilde{N}} \\
\stackrel{\infty}{\hat{N}}\end{array}$ & 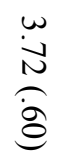 & 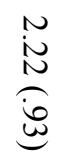 & $\begin{array}{l}\stackrel{\omega}{\tilde{O}} \\
\hat{\hat{\sigma}}\end{array}$ & $\begin{array}{l}\dot{\omega} \dot{0} \\
\hat{d}\end{array}$ \\
\hline$\overbrace{\stackrel{\infty}{\infty}}^{\stackrel{\infty}{\sigma}}$ & $\begin{array}{l}\stackrel{\omega}{\tilde{\omega}} \\
\stackrel{\hat{\sigma}}{0}\end{array}$ & $\begin{array}{l}\stackrel{\omega}{\tilde{u}} \\
\stackrel{\tilde{\omega}}{\omega}\end{array}$ & $\begin{array}{l}\stackrel{N}{N} \\
\stackrel{N}{0} \\
\pm\end{array}$ & $\begin{array}{l}\stackrel{w}{\tilde{O}} \\
\stackrel{\hat{\sigma}}{0}\end{array}$ & $\underset{\stackrel{\omega}{\omega}}{\stackrel{\omega}{\hat{~}}}$ \\
\hline$\overbrace{\substack{\infty \\
\infty}}^{\stackrel{\infty}{\infty}}$ & $\begin{array}{l}\stackrel{\omega}{\dot{\omega}} \\
\stackrel{\infty}{\hat{g}}\end{array}$ & 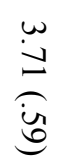 & 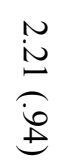 & 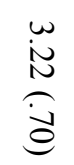 & $\underset{\stackrel{\omega}{\sigma}}{\stackrel{\omega}{\hat{d}}}$ \\
\hline$\underset{\stackrel{\vec{J}}{\vec{N}}}{\stackrel{\vec{N}}{\widehat{N}}}$ & $\begin{array}{l}\stackrel{\omega}{\tilde{n}} \\
\stackrel{\hat{~}}{\sigma}\end{array}$ & $\begin{array}{l}\stackrel{\omega}{\sigma} \\
\stackrel{\vec{G}}{\hat{\sigma}}\end{array}$ & $\underset{\stackrel{N}{\infty}}{\stackrel{\sim}{\infty}}$ & $\begin{array}{l}\stackrel{\omega}{\infty} \\
\stackrel{\infty}{\hat{j}}\end{array}$ & $\begin{array}{l}\stackrel{\omega}{\vec{y}} \\
\stackrel{\hat{\Phi}}{ \pm}\end{array}$ \\
\hline 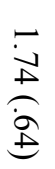 & 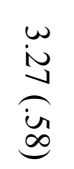 & $\underbrace{\stackrel{\omega}{\infty}}_{\stackrel{\omega}{\infty}}$ & 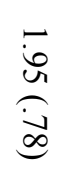 & $\begin{array}{l}\stackrel{\omega}{\vec{d}} \\
\stackrel{\dot{\omega}}{d}\end{array}$ & $\begin{array}{l}\stackrel{\omega}{\vec{H}} \\
\stackrel{+}{\hat{\omega}} \\
\dot{\infty}\end{array}$ \\
\hline$\frac{\vec{\infty}}{\stackrel{\infty}{\hat{N}}}$ & 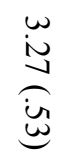 & $\stackrel{\omega}{\stackrel{\omega}{\hat{~}}}$ & 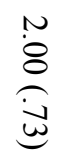 & 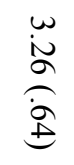 & $\begin{array}{l}\stackrel{\omega}{\dot{d}} \\
\stackrel{\hat{\sigma}}{0}\end{array}$ \\
\hline 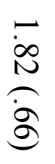 & 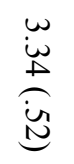 & 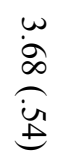 & $\begin{array}{l}\vec{\delta} \\
\stackrel{\sigma}{\sigma}\end{array}$ & $\begin{array}{l}\stackrel{w}{\tilde{O}} \\
\stackrel{\dot{u}}{u}\end{array}$ & $\begin{array}{l}\stackrel{\omega}{\ddot{0}} \\
\stackrel{0}{\dot{\omega}} \\
\dot{\infty}\end{array}$ \\
\hline$\frac{\vec{\delta}}{\hat{d}}$ & $\begin{array}{l}\stackrel{\omega}{ \pm} \\
\stackrel{\vec{t}}{\mathbb{b}}\end{array}$ & $\underset{\dot{a}}{\stackrel{\omega}{\hat{i}}}$ & $\stackrel{\stackrel{N}{\vec{\Xi}}}{\widehat{\Xi}}$ & $\begin{array}{l}\stackrel{w}{\tilde{U}} \\
\hat{\infty}_{\tilde{U}}\end{array}$ & $\begin{array}{l}\stackrel{w}{\dot{\omega}} \\
\stackrel{\dot{j}}{j}\end{array}$ \\
\hline$\overbrace{\stackrel{\infty}{\infty}}^{\overrightarrow{\hat{\sigma}}}$ & $\underset{\stackrel{w}{w}}{\stackrel{w}{\vec{d}}}$ & $\underset{\hat{\sigma}}{\vec{\sigma}}$ & $\stackrel{\stackrel{N}{\Xi}}{\stackrel{N}{N}}$ & $\begin{array}{l}\underset{\tilde{N}}{\infty} \\
\hat{i}_{\tilde{N}}^{\infty} \\
\infty\end{array}$ & $\begin{array}{l}\omega \\
\hat{\sigma} \\
\hat{\sigma}\end{array}$ \\
\hline$\stackrel{\vec{\Delta}}{\overrightarrow{\hat{\sigma}}}$ & 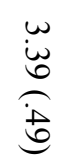 & $\underbrace{\stackrel{w}{\omega}}_{\vec{w}}$ & 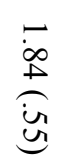 & $\stackrel{\omega}{\stackrel{\omega}{\hat{\sigma}}}$ & 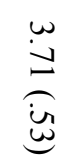 \\
\hline
\end{tabular}


The final model, including growth curves of commitment, in-depth exploration, and reconsideration, and associations between these dimensions, had a good fit $\left(\chi^{2}(302)=539.72\right.$, $p<.001 ; \mathrm{CFI}=.97$; TLI $=.96$; RMSEA $=.05$ ). Estimated means of intercepts and slopes are displayed in Table 7.2. Since especially the curvilinear pattern of reconsideration (which is a combination of a linear and quadratic slope) is hard to judge from a table, growth curves of commitment, in-depth exploration, and reconsideration are also plotted for boys (Figure 7.1a) and girls (Figure 7.1b).

Levels of commitment were stable throughout the entire adolescent period. For early to middle and middle to late adolescent boys and girls, slopes of commitment never reached significance. There were no gender differences in intercepts of commitment.

For in-depth exploration, our hypothesis was confirmed as increases were found. Levels of in-depth exploration were stable in early to middle adolescent boys and girls, but increased significantly in a linear fashion for middle to late adolescent boys and girls. Chisquare difference tests revealed that girls displayed higher levels of in-depth exploration when compared to boys throughout the entire period of adolescence. Boys and girls exhibited similar change rates.

Development of reconsideration was characterized by a curvilinear pattern that was different for early to middle adolescent boys and girls, and middle to late adolescent boys and girls (chi-square difference test: $p<.05$ ). In early to middle adolescence, girls displayed lower levels of reconsideration than boys. However, because boys exhibited significant curvilinear decreases in reconsideration whereas girls did not change significantly, the initial differences between boys and girls became smaller towards middle adolescence. In middle adolescence, gender differences initially became larger as girls did not change significantly, whereas boys increased in reconsideration. However, the increase for boys in middle adolescence was followed by a sharp decrease in late adolescence. Since girls' levels of reconsideration remained stable from middle to late adolescence, gender differences in reconsideration became much smaller in late adolescence. 


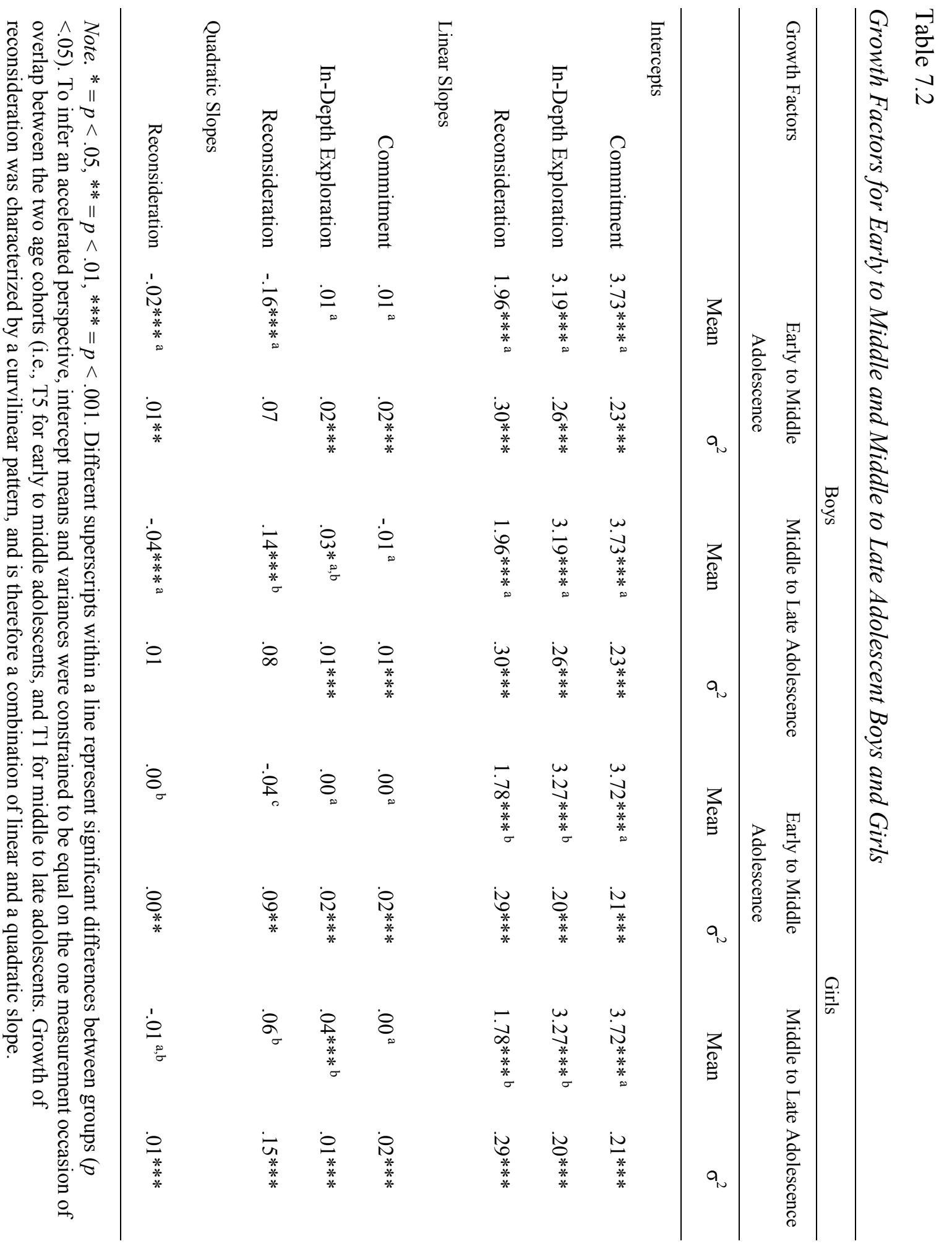


Chapter 7
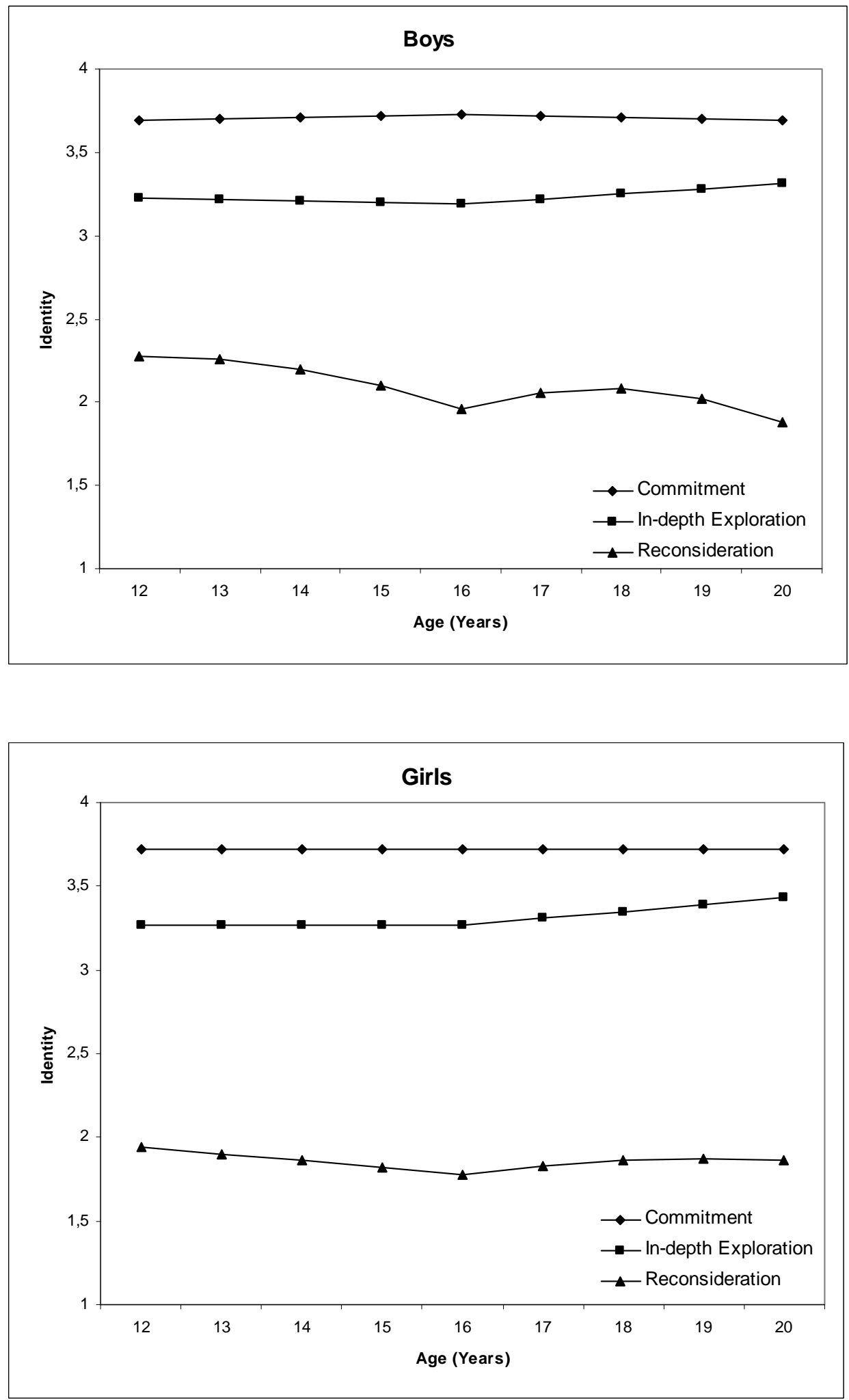

Figure 7.1. Estimated growth of commitment, in-depth exploration, and reconsideration in boys (a) and girls (b). 


\section{Rank-order Stability of Identity Dimensions}

Pearson correlations were calculated to assess the test-retest rank-order stability of identity dimensions (commitment, in-depth exploration, and reconsideration) across the five annual waves of this study, for boys and girls separately (see Table 7.3). We tested for significance of differences in rank-order stability between the four groups distinguished in the current study (i.e., early to middle and middle to late adolescent boys and girls). For that purpose, correlation coefficients were first transformed to $z$-scores using Fisher's $r$-to- $z$ transformation, and these $z$-scores were compared.

Table 7.3

Rank-order Stability and Profile Similarity of Identity Dimensions

\begin{tabular}{|c|c|c|c|c|}
\hline & \multicolumn{2}{|c|}{ Boys } & \multicolumn{2}{|c|}{ Girls } \\
\hline & Early to Middle & Middle to Late & Early to Middle & Middle to Late \\
\hline & Adolescence & Adolescence & Adolescence & Adolescence \\
\hline Commitment & $.38 * * *^{\mathrm{a}}$ & $.39 * * * a, b$ & $.26 * * * b$ & $.31 * * * a, b$ \\
\hline In-depth Exploration & $.37 * * * \mathrm{a}$ & $.38 * * *$ a & $.27 * * * \mathrm{a}$ & $.29 * * * \mathrm{a}$ \\
\hline Reconsideration & $.39 * * * \mathrm{a}$ & $.24 * * a, b$ & $.26 * * * b$ & $.34 * * * a, b$ \\
\hline Profile Similarity & $.73^{\mathrm{a}}$ & $.83^{\mathrm{b}}$ & $.82^{\mathrm{b}}$ & $.85^{\mathrm{b}}$ \\
\hline
\end{tabular}

Note. $*=p<.05, * *=p<.01, * * *=p<.001$. Different superscripts within a line represent significant differences between groups $(p<.05)$.

These analyses revealed that test-retest correlations of identity in early to middle adolescence did not significantly differ from test-retest correlations in middle to late adolescence, for both boys and girls. We did find gender differences in rank-order stability in early to middle adolescence, as rank-order stability was significantly higher for boys than for girls for commitment ( $r=.38$ and $r=.26$, for boys and girls respectively) and reconsideration ( $r=.39$ and $r=.26$, for boys and girls respectively).

\section{Profile Similarity in Identity Formation}

Profile similarity was measured by calculating $q$-correlations (e.g., Roberts et al., 2001) over the four-year period of this study for early to middle and middle to late adolescent boys and girls (see Table 7.3). Average $q$-correlations were high, demonstrating high profile similarity in early to middle and the middle to late adolescent boys and girls. Furthermore, we found a gender by age cohort interaction in increases of $q$-correlations. More specifically, boys had significantly lower levels of profile similarity in early to middle adolescence when compared to girls $(t(853.210)=-2.858, p=.004, d=-.201)$. Since boys' levels of profile similarity increased in middle to late adolescence $(t(401.594)=-2.645, p=.008, d=-.227)$ 
whereas girls exhibited no further increases, gender differences in profile similarity were no longer present in middle to late adolescence.

\subsection{Discussion}

Erikson $(1972,1974)$ described the formation of an unambiguous identity as the central developmental task of adolescence. The main purpose of the current study was to provide a comprehensive view on identity formation in adolescence by examining three types of change and stability, using a five-annual-wave longitudinal design. A three-dimension model of identity formation (Crocetti, Rubini, \& Meeus, 2008) was employed. In general, our findings were consistent with previous studies (e.g., Luyckx et al., 2008; Luyckx, Goossens, \& Soenens, 2006; Meeus, Iedema, Helsen, \& Vollebergh, 1999; Van Hoof, 1999; Waterman, 1999), as we found evidence for stability but also for small progressive developmental changes as adolescents grew older. These findings applied to both boys and girls.

\section{Stability and Change in Identity Formation through Adolescence}

Levels of commitment remained stable throughout the entire period of adolescence, for both boys and girls. Previous studies either found small increases (Meeus et al., 1999) or small decreases (Luyckx, Goossens, \& Soenens, 2006; Luyckx et al., 2008). Thus, if previous studies and the present study are taken together, the best conclusion with regard to commitment is that there appear to be no meaningful changes across time. As rank-order stability of commitment was quite high in both early to middle and middle to late adolescent boys and girls, this mean-level stability should be interpreted as a normative trend (e.g., Roberts \& DelVecchio, 2000) that applied to a vast majority of adolescents. Thus, results for commitment provide support for Van Hoof's (1999) concept of stability in identity formation. With regard to gender differences in commitment, we found that rank-order stability of boys was higher than for girls in early to middle adolescence. Further gender differences were not found for commitment.

Additionally, our results demonstrated that adolescents' current commitments were increasingly more explored as they grew older, as we found increases for in-depth exploration in middle to late adolescent boys and girls. Similar findings have been obtained in previous studies (Luyckx, Goossens, \& Soenens, 2006; Luyckx et al., 2008). Mean levels of in-depth exploration were stable in early to middle adolescent boys and girls. Similar to our results concerning commitment, rank-order stability did not increase with age in adolescence. No 
gender differences in rank-order stability of in-depth exploration were found, but mean-levels of in-depth exploration were somewhat higher for girls than for boys throughout adolescence. In-depth exploration involves reflection on one's current commitments. Since girls have been shown to exhibit higher levels of self-reflection in general (Burwell \& Shirk, 2007), it is perhaps not too surprising that they exhibit higher levels of reflection on current commitments. Levels of rank-order stability for in-depth exploration were quite high, which indicates that mean-level changes applied to a majority of individuals. Thus, middle to late adolescence is a period where most boys and girls start to explore their commitments in an increasingly active manner.

For reconsideration, we found profound gender differences. Throughout adolescence, girls displayed lower levels of reconsideration when compared to boys. These gender differences can be explained from an evolutionary point-of-view. Compared to boys, girls have a stronger preference to hold on to existing social bonds (Geary, Byrd-Craven, Hoard, Vigil, \& Numtee, 2003). High levels of reconsideration in the interpersonal domain would threaten social bonds directly, and reconsideration in the educational domain would threaten these bonds indirectly since much of an adolescents' social network is embedded in their school. As such, high levels of reconsideration could be particularly maladaptive for girls and less so for boys. This could be the reason why girls exhibit lower levels of reconsideration when compared to boys.

Besides gender differences in levels of reconsideration, there were also gender differences in change rates. Boys exhibited mean-level decreases in early to middle adolescence, whereas girls did not exhibit any significant changes throughout adolescence. Boys' decreases were only small in early adolescence, but became increasingly larger towards middle adolescence. These findings suggest that early to middle adolescence is a period where boys become increasingly more certain about their commitments. In middle to late adolescence, boys first display a small increase in reconsideration, but this increase is followed by a decrease. Despite this temporary increase for boys, their mean levels of reconsideration in middle to late adolescence remained below their mean levels in early adolescence. As there were no age-related changes in the relatively high levels of rank-order stability we found in early to middle adolescent boys and girls, the developmental patterns we found should be perceived as normative developmental trends (e.g., Roberts \& DelVecchio, 2000). The normativity of the slight, temporary, increase in reconsideration for boys is underscored by the fact that it coincides with a normative transition, as adolescents advance from high school to university when they are approximately 16 to 18 years old. They end up 
in a new school environment and meet new people. Their friends might go to a different university, which is likely to affect friendships. As a result, adolescents might need to reevaluate their commitments to their old friends, and look for new ones. Entering a new form of education is also likely to affect the educational identity domain. Adolescents need to find out what their new education means for them. Hence, they need to explore and find out whether their choice for a certain major as well as a certain university suites their needs. For this purpose, they might need to compare their current education with several possible alternatives. Thus, if the developmental context of 16 to 18 year old Dutch adolescents is taken into account, an increase in reconsideration should not necessarily be considered maladaptive. Our findings therefore underscore the importance of considering the context in which identity formation occurs (e.g., Bosma \& Kunnen, 2008). These results further demonstrate that our reconsideration scale is sensitive to changes in identity formation during normative transitions (i.e., the transition from high school to university), and thereby underscore the validity and importance of this relatively new identity dimension. What is unclear, however, is why the temporary increase in reconsideration only occurs for boys, especially since a previous study (Luyckx et al., 2008) demonstrated a similar curvilinear pattern for an identity dimension closely related to reconsideration (i.e., exploration in breadth) in a female college sample. It could be argued that the girls in the current study already possessed a more mature identity (indicated by higher levels of in-depth exploration and lower levels of reconsideration) by middle adolescence, when compared to boys. Girls' more mature identities should be indicative of a heightened sense of sameness and continuity across social contexts (Erikson, 1972, 1974). As a result, they could perceive less of a need to adapt their identities in a new social context (i.e., university) and do not start to doubt their commitments when entering universities.

Across dimensions, levels of rank-order stability were quite high in both early to middle and middle to late adolescence. These findings indicate that individual differences in identity dimensions are already set to a large extent in early to middle adolescence, and do not become more set as adolescents grow older. We did find some gender differences in early to middle adolescence, as boys exhibited significantly higher levels of rank-order stability for commitment and reconsideration, when compared to girls. All the same, our results with regard to the three separate identity dimensions indicate that identity formation is characterized by stable individual differences across dimensions and stable mean levels of commitment, but with progressive mean-level changes for in-depth exploration in both boys and girls, and in reconsideration for boys. Thus, the current study indicates that identity 
commitments are increasingly better explored by both boys and girls (indicated by increases for in-depth exploration), while certainty about commitments is already high for girls in early adolescence, and increases for boys throughout adolescence (indicated by decreases in reconsideration). Taken together, our findings are not necessarily indicative of identity status changes, but they do suggest that adolescents move towards an achieved identity status. Therefore, our results support Waterman's $(1982,1999)$ concept of progressive change. Although the consistent overall pattern indicating progressive changes suggests that the changes we found are much more than just fluctuations in identity formation, the average change rate for the various identity dimensions was still rather small. As such, van Hoof's (1999) concept of stability also receives some support in the current study.

We found that profiles including all three identity dimensions distinguished in the current study (i.e., commitment, in-depth exploration, and reconsideration) were already quite stable for early to middle adolescent girls and did not become more stable for them in middle to late adolescence. Boys, on the other hand, had a less stable identity profile than girls in early to middle adolescence, but since their identity profile became much more stable in middle to late adolescence, they caught up with girls again. We found a similar pattern with regard to mean-level changes in reconsideration (i.e., boys initially had higher levels of reconsideration than girls, but displayed stronger decreases). Thus, similar to previous studies (Kroger, 1997; Meeus et al., 1999; Waterman, 1982, 1993, 1999), we found rather small overall gender differences in identity formation. However, there are gender differences in the timing of change. More specifically, girls seem to be ahead on boys in identity formation in early to middle adolescence, with boys catching up again in middle to late adolescence. Thus, girls seem to mature earlier with regard to identity formation than boys. Evidence for similar gender differences in the timing of developmental changes have not only been previously reported for identity formation (Kroger, 1997), but also in the field of adolescent personality research (Klimstra, Hale, Raaijmakers, Branje, \& Meeus, in press). Gender differences in timing of maturation on psychological variables could be caused by similar gender differences in biological maturation, as girls are typically ahead on boys in pubertal timing (e.g., Petersen, Crockett, Richards, \& Boxer, 1988) and neurological development (Giedd et al., 1999). Future studies could investigate whether biological processes are indeed the cause of gender differences in the timing of adolescent identity formation.

Taken together, our findings reflect progressive changes in identity formation that apply to a majority of adolescents (as evidenced by relatively high levels of rank-order stability). The fact that the changes we found occurred in the relatively new identity 
dimensions in-depth exploration and reconsideration fits into our conceptualization of identity formation in adolescence. We propose that identity formation in adolescence is not specifically characterized by longitudinal increases in commitments themselves, but by increasing reflection on and certainty about commitments.

When taken as a whole, our results contribute to the debate on the timing of identity formation. Our results indicate that changes in identity formation take place both in early to middle adolescence, as predicted by Meeus (1996), and in middle to late adolescence as predicted by Marcia (1980) and Waterman $(1982,1993)$. Thus, the amount of change is not different in early to middle adolescence and middle to late adolescence, but differences between identity formation in early to middle adolescence and middle to late adolescence are related to specific dimensions. Findings for reconsideration suggested that early to middle adolescence is characterized by small increases in certainty about commitment for boys, but findings for in-depth exploration clearly indicated that middle to late adolescence is the period where commitments become more actively explored, for both boys and girls. Therefore, our results fit within the dual-cycle model proposed by Luyckx, Goossens, and Soenens (2006). In their model, the first cycle, referred to as "the commitment formation cycle", represents the formation of stable commitments. We found some evidence for such a process in our data, reflected by mean-level decreases in reconsideration in early to middle adolescent boys. The second cycle, "the commitment evaluation cycle", involves actively thinking and evaluating present commitments. Evidence for this cycle is clearly represented in our data by increasing mean levels of in-depth exploration in middle to late adolescent boys and girls.

Some limitations of the current study should be noted. In this study, we focused on long-term changes. Identity formation is assumed to be characterized by inner conflicts or crises (Erikson, 1972, 1974) that are hard to detect in studies with one-year intervals between measurements. In addition, the three-dimension model employed in the current study offers a dynamic conceptualization of identity formation (Crocetti, Rubini, \& Meeus, 2008). However, with large intervals between measurement occasions the exact dynamics of identity development can not be captured adequately (Luyckx, Goossens, \& Soenens, 2006; Lichtwarck-Aschoff, van Geert, Bosma, \& Kunnen, 2008). To obtain a detailed perspective on the dynamics of identity formation, future studies should include more frequent measurements.

In this study of growth and stability in identity formation, our focus was on change in three identity dimensions. To disentangle the causes of inter-individual differences in identity formation, future studies should also address long-term associations of identity dimensions or 
statuses with other factors that have been shown to be related in cross-sectional studies, or should be theoretically related. For example, Luyckx, Soenens et al. (2006) already found strong evidence for a longitudinal interplay between personality and identity, but merely assessed late adolescent women. In another longitudinal study, Kroger and Haslett (1988) demonstrated that identity formation predicted attachment style. However, they only employed a small sample. Further research revealed cross-sectional links between identity dimensions on the one hand, and psychosocial problems (Crocetti, Rubini, \& Meeus, 2008) and the separation-individuation process (Meeus, Iedema, Maassen, \& Engels, 2005) on the other hand. Taken together, the longitudinal associations between identity formation and factors like personality, separation-individuation (e.g., attachment), and psychosocial problems are not well-established. Future studies should aim to examine the relations between identity formation and these factors more thoroughly.

The current study also showed the importance of considering gender in identity formation, as girls exhibited more stability and maturity at the early stages of identity formation with boys catching up in late adolescence. Our findings regarding temporary increases in reconsideration for boys during the transition from high school to university already showed that it is also important to consider the developmental context in which identity formation occurs (Bosma \& Kunnen, 2008). Since these temporary increases in reconsideration only occurred for boys, our findings reveal that not only the main-effects of gender and context exert an influence on identity formation. In line with Kroger's recommendations (Kroger, 1997), our results point towards the importance of studying the effect of gender by context interactions. Thus, future studies should assess the effects of gender, contextual factors, and the interactions of gender by context on identity formation whenever it is feasible.

In conclusion, the current study reveals that identity formation is described by both stability and progressive changes. No age-related changes were found with regard to rankorder stability of identity dimensions and mean levels of commitment. There was, however, evidence for progressive change, especially for boys. Boys gained an increasingly more stable identity profile (evidenced by increases in profile similarity), displayed a decreasing tendency for reconsideration throughout adolescence, and revealed increases in in-depth exploration in middle to late adolescence. Girls also exhibited progressive changes, as they displayed strong increases for in-depth exploration. Taken together, our findings indicate that identity formation is characterized by progressive changes in dealing with commitments, rather than by changes in commitments themselves. Therefore, our findings demonstrate the merits of a 
three-dimension approach to identity formation, with an emphasis on the relatively new identity dimensions of in-depth exploration and reconsideration. 


\section{Chapter 8}

Short Term Fluctuations in Identity:

Introducing a Micro-level Approach to Identity Formation ${ }^{8}$

${ }^{8}$ Klimstra, T. A., Luyckx, K., Hale, W. W., Frijns, T., Van Lier, P. A. C., \& Meeus, W. H. J. (in press). Short-term fluctuations in identity: Introducing a micro-level approach to identity formation. Journal of Personality and Social Psychology. 


\begin{abstract}
The present study was aimed at examining one relatively neglected part of the identity formation process: the short-term dynamics of identity formation. The short-term dynamics were assessed by examining (a) the day-to-day course of two key dimensions of identity formation (i.e., commitment and reconsideration), and (b) the impact of fluctuations in commitment and reconsideration on subsequent levels of these two dimensions. Longitudinal data on 580 early adolescents $(54.8 \%$ boys, $45.2 \%$ girls) was employed to test these assertions. We found evidence for a commitment-reconsideration dynamic that operated on a day-to-day basis. Furthermore, our findings confirmed Erikson's (1950) assertion that identity reflects a sense of sameness and continuity as a more stable identity (reflected by little day-today fluctuations) was predictive of higher levels of commitment and lower levels of reconsideration. Taken together, the current study underscores the importance of the shortterm dynamics of identity formation
\end{abstract}




\subsection{Introduction}

The formation of a stable identity is the single most important developmental task of adolescence. Identity formation begins in early adolescence, when parental norms and values are increasingly questioned and a search for a self-defined set of norms, values, and commitments begins (Erikson, 1950). For the last four decades, identity research has been dominated by Marcia's elaboration on Erikson's writings, that is, the identity status paradigm (Marcia, 1966; for an overview, see Meeus, Iedema, Helsen, \& Vollebergh, 1999). Although several studies inspired by the identity status paradigm have addressed long-term developmental processes in identity formation (e.g., Luyckx, Goossens, \& Soenens, 2006; Meeus et al., 1999), little is known about the short-term dynamics involved in identity formation (Lichtwarck-Aschoff, van Geert, Bosma, \& Kunnen, 2008; Schwartz, 2001). Consequently, the purpose of the current study is to unravel the course and the implications of short-term processes in identity formation, by focusing on the day-to-day dynamics of identity commitment and reconsideration of commitment (being two crucial identity formation dimensions), and the role and function of day-to-day fluctuations in the levels of these two identity dimensions. For these purposes, data on 580 adolescents partaking in an ongoing longitudinal study in the Netherlands were used.

\section{Identity Formation in Adolescence: Theoretical Assumptions and Empirical Findings}

The period of adolescence marks the transition from childhood to adulthood. In this period, individuals need to gradually shift from relying on their childhood identifications (i.e., their parents' teachings) to developing their own values and ethics (Erikson, 1950). In other words, individuals need to establish a new sense of sameness and continuity after they have refuted their childhood identifications. For this purpose, they need to develop a stable set of norms, values, and commitments as a frame of reference to guide them in their day-to-day activities. This sense of sameness and continuity is referred to as an identity (Erikson, 1950). According to Erikson, an individual's identity can be described along a continuum, ranging from "role confusion" (i.e., total absence of a stable identity) to "identity synthesis" (i.e., a successful accomplishment of an identity).

Erikson wrote about identity in a clinical and figurative sense, but his theory was somewhat lacking in precision and detail (Côté \& Levine, 1987). Marcia (1966) was among the first to elaborate on Erikson's writings by introducing a paradigm that could guide empirical research on identity formation: the identity status paradigm. In the identity status 
paradigm, the focus is on two key processes or building blocks of identity formation: exploration and commitment. Whereas exploration refers to the comparison of several alternatives in identity-defining domains, commitment denotes the selection of certain alternatives and the engagement in relevant activities towards the implementation of these choices.

In the current study, we used two dimensions (i.e., reconsideration and commitment) adopted from a recently developed identity formation model (Crocetti, Rubini, \& Meeus, 2008). Whereas the commitment dimension within this model of identity formation is highly similar to the conceptualization of commitment in Marcia's (1966) status paradigm, the reconsideration dimension diverts slightly from Marcia's exploration dimension. Unlike Marcia's exploration dimension, reconsideration refers to a continuous process of comparing present commitments to alternative ones. Therefore, the model by Crocetti, Rubini, \& Meeus (2008) implies the continuous presence of at least a minimal sense of commitment in relevant ideological and interpersonal identity domains. Hence, the identity formation model described by Crocetti, Rubini, and Meeus (2008) captures the essence of Erikson's assertion (1950), that "in puberty and adolescence all samenesses and continuities relied on earlier are more or less questioned again” (pp. 252-253). Thus, Crocetti, Rubini, and Meeus' (2008) conceptualization of reconsideration can be perceived as being close to Erikson's (1950) description of identity formation. The inclusion of such a reconsideration dimension also allows to better capture the identity versus role confusion dynamic described by Erikson (1950), as reconsideration and commitment reflect two opposing forces. Commitment indicates a sense of security, whereas reconsideration represents the opposing force that questions this security (Meeus, van der Schoot, Keijsers, Schwartz, \& Branje, 2010).

In conclusion, research on identity formation has evolved from Erikson's (1950) writings through Marcia's (1966) empirical conceptualization towards refined processoriented conceptualizations of identity formation (e.g., Crocetti, Rubini, \& Meeus, 2008). Throughout this evolution, only a limited number of studies have examined the development of commitment and exploration processes. A short overview of these longitudinal studies will now be provided.

\section{Capturing the Dynamics of Identity Formation: The Need for a Short-Term Approach}

In recent years, the knowledge on the dynamics of identity formation has increased rapidly. The most recent elaboration on identity formation theory, the dual-cycle model by Luyckx and colleagues (Luyckx et al., 2006), has been derived directly from their longitudinal 
research. Using longitudinal data on a sample of predominantly female college students, they were able to demonstrate that identity formation is guided by intertwined commitmentformation and commitment-evaluation cycles. In the commitment-formation cycle, various possible commitments are weighted against one another before an individual chooses one of these possible alternatives. As such, the previously mentioned identity versus role confusion (i.e., certainty-uncertainty) dynamic (Erikson, 1950; Meeus et al., 2010) can be considered to be at the heart of this commitment-formation cycle.

The commitment-evaluation cycle focuses on finalizing identity commitments for the present moment, as it mainly entails the evaluation of current commitments. Commitmentevaluation partially requires the ability to actively reflect on oneself. The ability to engage in such abstract thought-processes with regard to the self is not yet fully developed in early adolescence, and only emerges as the adolescent grows older (e.g., Harter, 1990). For that reason, early adolescents are likely to be mainly involved in commitment-formation processes and less in commitment-evaluation processes (Bosma \& Kunnen, 2008).

In line with these theoretical assumptions, Klimstra, Hale, Raaijmakers, Branje, and Meeus (2009) recently demonstrated that early to middle adolescents were characterized by low and stable levels of in-depth exploration, which is the identity dimension that is typically associated with commitment-evaluation (Luyckx et al., 2006). Only in middle to late adolescence do individuals start to reflect more deeply on their commitments. Klimstra et al. (2009) did find strong evidence for the presence of commitment-formation processes (and hence for a commitment-reconsideration dynamic) in early to middle adolescence, as adolescents started to reconsider their commitments less often as they grew older. As such, the current study, which samples early adolescents, focuses on the two dimensions (commitment and reconsideration) that represent the certainty-uncertainty dynamic within the commitment-formation process.

The dual-cycle model (Luyckx et al., 2006) offers a dynamic conceptualization of the identity formation process, much like the related certainty-uncertainty dynamic proposed by Meeus and colleagues (2010). However, longitudinal studies that examined changes in the identity dimensions comprising these dynamic conceptualizations of identity formation have employed designs with relatively long-term intervals between measurement occasions (i.e., 6 months up to 1 year). Studies with such long-term intervals merely provide a rough estimate on how the dynamics of identity formation operate. Identity formation processes operating across long-term intervals are referred to as macro-level processes (Lichtwarck-Aschoff et al., 2008). Unfortunately, such work at the macro-level is not informative on how the 
developmental processes exactly operate on a daily level (Grotevant, 1987; LichtwarckAschoff et al., 2008). Because identity has been described as a dynamic and self-organizing system (Kunnen, Bosma, Van Halen, \& Van der Meulen, 2001), a micro-level approach has been recommended to focus in depth on identity formation processes. In micro-level approaches, processes are measured on a day-to-day basis (Lichtwarck-Aschoff et al., 2008). The current study aims to offer a detailed perspective on identity formation by tracking early adolescent identity formation on such a day-to-day basis.

Micro-level processes should not be studied in isolation. Instead, it has been argued that micro-level processes should be related to macro-level processes (Lichtwarck-Aschoff et al., 2008). Such an approach allows to test one of the key assumptions of identity theory, namely Erikson's $(1950,1968)$ tenet that identity formation primarily involves a search for sameness and continuity. More specifically, Erikson suggests that individuals with a more stable sense of sameness and continuity possess a stronger identity. As such, one could argue that individuals dealing with identity issues in a consistent way (i.e., experiencing sameness and continuity in the way one deals with identity issues) should have a stronger identity in general. For that reason, it is important to examine whether day-to-day fluctuations in the identity dimensions of commitment and reconsideration (i.e., micro-level processes) would be predictive of subsequent levels on these identity dimensions (i.e., macro-level processes). Unfortunately, to the best of our knowledge, such endeavours have not been undertaken in the field of identity research. In the current study, we will explore if stability of commitment and reconsideration is predictive of subsequent levels of these two identity dimensions.

\section{From a Global towards a Domain-Specific Approach to Identity Formation}

Considering short-term dynamics instead of long-term processes is only one way to obtain a more detailed perspective on identity formation. Considering specific identity domains instead of global measures combining different identity domains is another way to obtain a more detailed perspective on identity formation. Because identity formation has been shown to follow a different course in different domains, a focus on separate identity domains has been recommended (Goossens, 2001). Following these recommendations, we suggest that short-term processes should be studied within specific identity domains instead of at a global level.

Marcia (1966) singled out three domains with particular relevance towards identity formation: occupation, religion, and politics. These domains primarily tapped issues of 
ideological identity, whereas it is apparent from Erikson's writings that identity formation also involves interpersonal issues or domains. For that reason, Grotevant, Thorbecke, and Meyer (1982) extended Marcia's identity status paradigm into the interpersonal domain, tapping into the content areas of friendships, dating, and sex roles. A similar distinction between ideological and interpersonal domains has been adopted in several identity measures, such as the Extended version of the Objective Measure of Ego-Identity Status (EOM-EIS; e.g., Bennion \& Adams, 1986), the Ego Identity Process Questionnaire (EIPQ; Balistreri, Busch-Rossnagel, \& Geisinger, 1995), the Utrecht-Groningen Identity Development Scale (U-GIDS; Meeus, 1996), and the Utrecht Management of Identity Commitments Scale (UMICS; Crocetti, Rubini, \& Meeus, 2008).

Unfortunately, a domain-specific approach has only been applied in two studies examining developmental changes in exploration and commitment dimensions (Meeus, Iedema, \& Maassen, 2002; Meeus, Iedema, Maassen, \& Engels, 2005). In the first study, Meeus et al. (2002) demonstrated that, across a three-year interval, commitment predicted exploration positively, and exploration predicted commitment positively. However, this was only the case with regard to the interpersonal domain and not to the ideological domain. The second study (Meeus et al., 2005) revealed that cross-sectional age-related increases in exploration were quite similar for interpersonal and ideological identity. However, age-related increases in commitment were much stronger for interpersonal identity than for ideological identity. Thus, developmental processes in interpersonal domains differed from those in ideological domains.

These differences between specific identity domains have been explained in previous work by Meeus et al. (1999). They proposed a distinction between open and closed domains of adolescent identity formation. In closed domains, such as education, adolescents may find continuous reflection on their commitments not that useful, because they can exert little influence on them. Especially in early adolescence, adolescents can either commit themselves to their high school education or not, as real alternatives are not readily available. That is, they may question their commitment to school, but the motivation to fully engage in exploration of their commitment to their education is likely to be low, as such exploration activities could not possibly result in them making a choice for a completely different kind of education. This situation changes to some extent in college, where adolescents can switch from one major to another, but education in early adolescence should be considered as a closed domain.

In open domains, such as relational identity, much more alternatives are available and adolescents can freely explore several possible alternatives. The validity of this claim has 
been underscored in several studies, as more than $50 \%$ of adolescent friendships discontinue after one year (Branje, Frijns, Finkenauer, Engels, \& Meeus, 2007; Connolly, Furman, \& Konarski, 2000; Degirmencioglu, Urberg, Tolson, \& Richards, 1998). As such, they seem to be exploring several possible alternatives and, therefore, are quite likely to be engaged in identity formation processes in the friendship domain. For that reason, the previously described certainty-uncertainty dynamic (Meeus et al., 2010) should, at least among early adolescents, be more active in more open interpersonal domains (i.e., friendships) than in more closed ideological domains (i.e., education).

\section{The Present Research}

The current study was guided by three main objectives. These objectives are discussed in the upcoming sections.

Objective 1: Validation of a single-item measure. Until now, there have been no systematic attempts to measure the short-term dynamics of identity formation. For that reason, Meeus developed a single-item version based on an existing questionnaire (U-MICS; Crocetti, Rubini, \& Meeus, 2008). The original version is aimed at measuring identity formation processes at a macro-level (e.g., assessed on an annual basis). Because filling out a questionnaire on a day-to-day basis puts a heavy burden on respondents, we shortened the original version considerably. The adapted, single-item version tapped commitment and reconsideration each with one item per domain (e.g., one item for interpersonal commitment and one item for relational commitment).

Reliability and validity of such single-item measures can not be established with traditional techniques, such as Cronbach's alpha (for reliability) and factor-analysis (for validity). As such, we used techniques specifically designed to validate such single-item measures. These techniques, and the reliability and convergent and discriminant validity estimates they produced, will be discussed in the Results section. There, we will also discuss their correlations with measures of academic adjustment (i.e., to validate our ideological identity measures), and relationship quality with regard to the best friend (i.e., to validate our interpersonal identity measures). Generally, commitment is positively related to adjustment, and reconsideration is negatively related to adjustment (Crocetti, Rubini, \& Meeus, 2008). Therefore, ideological commitment should be positively related to school adjustment, whereas ideological reconsideration should be negatively related to school adjustment. In addition, interpersonal commitment should be positively related to support, and negatively related to negative interactions with regard to friendships, whereas interpersonal reconsideration should 
be negatively related to support, and positively related to negative interactions with regard to friendships.

\section{Objective 2: Capturing the day-to-day dynamics of identity formation. Identity} formation has been described as a dynamic and self-organizing system (Grotevant, 1987; Kunnen et al., 2001; Lichtwarck-Aschoff et al., 2008). Therefore, it is important to uncover how the day-to-day dynamics of identity formation work. For that reason, the second objective was to focus on these dynamics. We focused on one particularly important aspect for early adolescents: the certainty-uncertainty dynamic (Meeus et al., 2010). As identity formation in one domain can differ from identity formation in another domain (Goossens, 2001; Meeus et al., 1999), a domain-specific approach to identity formation was adopted. That is, we distinguished among one interpersonal domain (i.e., friendships) and one ideological domain (i.e., education). Meeus et al. (1999) suggested that interpersonal domains might be more open to change than ideological domains such as education, especially in early and middle adolescence. For that reason, we expected the certainty-uncertainty dynamic to be activated more strongly (i.e., with more reciprocal influences between commitment and reconsideration) in the friendship domain as compared to the educational domain.

Objective 3: Examining the implications of short-term fluctuations. As previously mentioned, identity formation mainly involves a search for sameness and continuity (Erikson, 1950). This, in turn, leads to the question whether day-to-day fluctuations in the identity dimensions of commitment and reconsideration (i.e., the degree to which one experiences sameness and continuity with regard to dealing with identity issues) are predictive of the overall strength of one's identity (indicated by subsequent mean-levels of commitment and reconsideration). Unfortunately, fluctuations in identity formation processes have not been studied thus far. In a related field studying processes with regard to the self, Kernis and colleagues (e.g., Kernis, Grannemann, \& Barclay, 1989, 1992) operationalized the instability of self-esteem as the magnitude of short-term fluctuations in levels of self-esteem. As a measure of these short-term fluctuations, they used intra-individual standard deviations of self-esteem scores across subsequent assessments.

In a series of studies, Kernis and colleagues have demonstrated the importance of considering the stability alongside the level of self-esteem. For example, they demonstrated that instability of self-esteem predicted a heightened tendency to experience anger and hostility, whereas they found no main-effect for levels of self-esteem (Kernis et al., 1989). Central to the purpose of the present study, an instable sense of self-esteem was also found to be related to lower levels of self-esteem (Campbell, 1990; Kernis et al., 1992). 
In the current study, we apply the method introduced by Kernis and colleagues to identity formation processes in examining the relation between the stability of commitment and reconsideration, and subsequent levels of these two identity dimensions. Put differently, we will explore whether Erikson's conceptualization of identity as a notion of sameness and continuity (Erikson, 1950, 1968) is indeed predictive of subsequent levels of commitment and reconsideration. For this purpose, we will again use a domain-specific approach.

\subsection{Method}

\section{Participants and Procedure}

Participants were 651 adolescents enrolled in an ongoing longitudinal project for Research on Adolescent Development And Relationships (RADAR). Of these 651, 626 were asked for participation in three internet measurement weeks per year. The remaining 25 participants, who had been selected for the study because they had been victims of violent acts, did not participate in internet assessments to limit the burden on these vulnerable adolescents.

In each week, participants were asked to fill out a questionnaire tapping into identity, aspects of relationships, and academic adjustment at a micro-level for five consecutive days. In the current study, we focus on identity at a micro-level. The initial internet assessment took place in June 2006, the second and third internet assessments were three and six months later, respectively. Nine months after the initial internet assessment, the first annual assessment of identity (i.e., at the macro level) took place. Of the 626 adolescents who were asked to participate in internet assessments, the 580 adolescents $\left(54.8 \%\right.$ boys; $45.2 \%$ girls; $M_{\text {age }}$ at the first internet measurement $=13.32(S D=0.53))$ that participated in at least one of the internet assessments and completed identity questionnaires at the subsequent annual measurement wave were included in the analyses. Analyses revealed that the 46 non-participating adolescents did not differ significantly from the 580 participating adolescents with regard to age, gender, or socioeconomic status.

Across all measurements, $13.3 \%$ of data was missing. These missing values were estimated in Mplus (Muthén \& Muthén, 2007) using the Full Information Maximum Likelihood (FIML) procedure. 


\section{Measures}

Commitment-Reconsideration Dynamic: Identity at the Macro Level. Commitment and Reconsideration were assessed with the Utrecht-Management of Identity Commitments Scale (U-MICS; Crocetti, Rubini, \& Meeus, 2008), a self-report measure based on the U-GIDS (Meeus, 1996). This instrument uses five-point Likert-scale items, with a response format ranging from 1 (completely untrue) to 5 (completely true). Five items measure commitment and 3 items reconsideration in each identity domain. As we studied one ideological and one interpersonal domain, 16 items were used to assess commitment and reconsideration. Sample items are: "My education/best friend gives me certainty in life" (ideological/interpersonal commitment) and "I often think it would be better to try and find different education/a different best friend" (ideological/interpersonal reconsideration). Validity and reliability of UMICS have been established for boys and girls, early and middle adolescents, and Dutch and ethnic minority youths (Crocetti, Rubini, \& Meeus, 2008; Crocetti, Rubini, Luyckx, \& Meeus, 2008). Cronbach's alphas were .94 for both ideological and interpersonal commitment, and .88 for both ideological and interpersonal reconsideration.

Commitment-Reconsideration Dynamic: Identity at the Micro Level. To measure identity on a daily basis (i.e., at the micro level), we used a single-item version of U-MICS developed by Meeus. Similar to the former version of U-MICS, five-point Likert-scale items with a response format ranging from 1 (completely untrue) to 5 (completely true) were used. Ideological (i.e., school) and interpersonal (i.e., relation with best friend) domains were assessed with one item for each dimension (i.e., commitment and reconsideration). The items were: "Today, I felt confident about myself because of my education/best friend" (ideological/interpersonal commitment) and "Today, I felt that I could better look for a different school/best friend" (ideological/interpersonal reconsideration). In order to make sure that participants really reported on their best friend and not on a romantic partner, we obtained the names of the best friends before the internet assessments. The section of the questionnaire including the single-items tapping commitment and reconsideration was preceded by an instruction in which we explicitly mentioned that the participants should answer questions about the person they indicated as their best friend. Thus, if their best friend would have been called John Smith the instruction would read: "You nominated John Smith as your best friend. When answering the following questions, keep this person in mind." Statistic concerning the reliability and validity of our single-item measures are reported in the Results section under the heading of Objective 1. 
Short-Term Fluctuations in Identity

\subsection{Results}

\section{Objective 1: Validation of the Single-Item Measure}

Reliability. To establish the reliability of the single-item version of U-MICS, we first assessed its internal consistency using a procedure developed by Heise (1969) (see also Robins, Hendin, \& Trzesniewski, 2001). In this procedure, reliability of single-item scales is assessed with an estimate of test-retest reliability that separates true change from measurement error, with the following equation: $r_{\mathrm{xx}}=\left(r_{12} * r_{23}\right) / r_{13}$. As there were five days within each internet week, we calculated three Heise estimates per identity dimension for each week. Because there were three weeks, nine Heise estimates were available for each identity dimension. Average reliability as estimated with the Heise coefficient was .65 (range $=.50-$ .76 ) for ideological and .74 (range $=.58-.83$ ) for interpersonal commitment, and .76 (range $=$ $.64-.87)$ for ideological and .87 (range $=.77-.94)$ for interpersonal reconsideration.

Convergent and Discriminant Validity. Validity was established by calculating convergent and discriminant correlations between the single-item micro-level measure and the standard measure of U-MICS. To measure convergent and discriminant validity, we correlated the three across-week means for each identity dimension with the corresponding (i.e., convergent validity) and the non-corresponding scale (i.e., discriminant validity) of the macro-level version. Week means and standard deviations of these means for micro-level identity, as well as means and standard deviations of macro-level identity, are displayed in Table 8.1.

Across-week means of ideological commitment correlated .33 (range $=.31$ to $.37 ; p$ 's $<.001)$ on average with ideological commitment in the macro-level measure, whereas the mean correlation with ideological reconsideration at the macro level was -.15 (range $=-.12$ to -.18; $p$ 's <.01). For interpersonal commitment, correlations with interpersonal commitment and reconsideration at the macro level were .35 (range $=.34$ to $.36 ; p$ 's $<.001)$ and -.17 (range $=-.16$ to $-.20 ; p$ 's $<.001$ ), respectively. In sum, our analyses revealed that across-week means for commitment in both identity domains were consistently stronger correlated with the corresponding commitment scale in the macro-level version than with the reconsideration scale. 


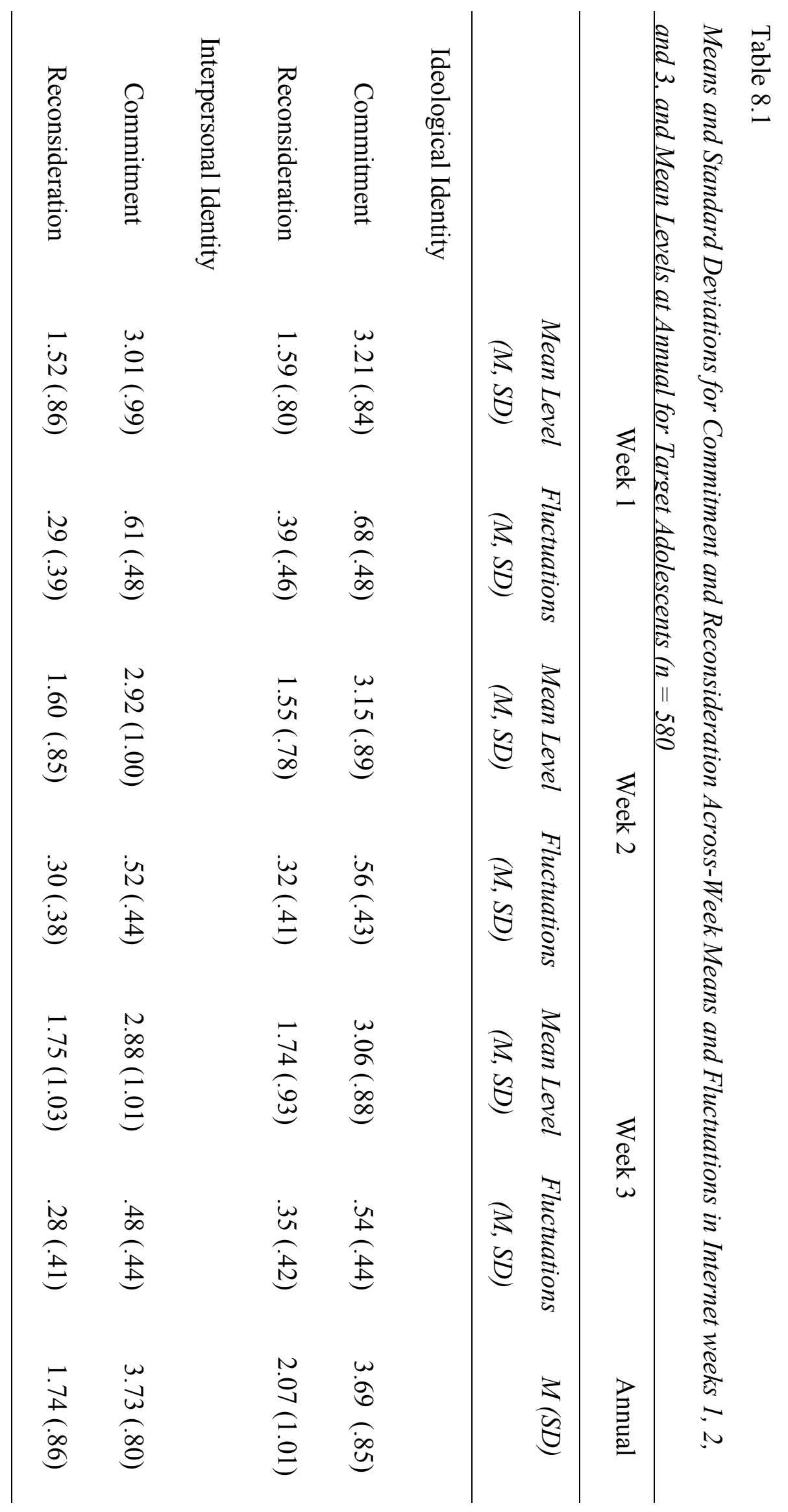


With respect to the validity of the state version of reconsideration in the ideological domain, across-week means correlated .38 (range $=.31$ to $.44 ; p$ 's $<.001)$ on average with reconsideration in the macro-level version, whereas the mean correlation with macro-level commitment was -.28 (range $=-.24$ to $-.32 ; p$ 's $<.001$ ). In the interpersonal domain, these correlations were $.21($ range $=.18$ to $.25 ; p$ 's $<.001)$ and $-.13($ range $=-.11$ to $-.15 ; p$ 's $<.05)$, respectively. Thus, across-week means of reconsideration correlated higher with the corresponding reconsideration scale in the macro-level version (i.e., convergent validity) than with the commitment scale in the macro-level version (i.e., discriminant validity), for both domains. Overall, our analyses confirm the convergent and discriminant validity of the singleitem version of U-MICS.

External Validity. Single-items tapping ideological commitment and reconsideration were externally validated by examining their correlations with items tapping academic adjustment (i.e., "In comparison with your classmates: how well was your school performance in the last week?", "In comparison with your classmates: how much did you like school?", "How often did you skip school in the last three months"). The item on school performance and the item tapping the degree to which adolescents enjoyed school were scored on a fifteen-point likert scale with a response format ranging from 0 ("much worse") to 15 ("much better"), and the item on skipping school was scored on a five-point likert scale with a response format ranging from 1 (“I haven't skipped school”) to 5 (“almost every day").

External validity of the single-items tapping interpersonal commitment and reconsideration was assessed by examining their correlations with two-item daily measures of support provided by the best friend (i.e., "How enjoyable was your relationship with [name of best friend] today?", "Did [name of best friend] show you that he/she cares about you?"), and negative interactions with the best friend (i.e., "Did you and [name of best friend] get annoyed with each other today?", "Did you and [name of best friend] hassle today?") adapted from the Network of Relationships Inventory (NRI; Furman \& Buhrmester, 1985, 1992). These items were scored on a 7-point likert scale with a response format ranging from 1 ("not at al") to 7 ("very").

Week means of ideological commitment correlated positively with school performance $($ mean correlation $=.17$; range $=.13$ to $.22 ; p$ 's $<.01)$ and the degree to which adolescents enjoyed school (mean correlation $=.31$; range $=.28$ to $.33 ; p$ 's $<.001$ ), and correlated negatively with the number of times adolescents skipped school (mean correlation $=-.16$; range $=-.20$ to $-.14 ; p$ 's $<.01$ ). For ideological reconsideration, week means correlated negatively positively with school performance (mean correlation $=-.17$; range $=-.24(p$ 
$<.001)$ to $-.09(p>.05))$ and the degree to which adolescents enjoyed school (mean correlation $=-.38$; range $=-.42$ to $-.31 ; p$ 's $<.001$ ), and positively with skipping school (mean correlation $=.13$; range $=.05(p>.05)$ to $.24(p<.001))$.

The relationship quality measures we used to externally validate our interpersonal commitment and reconsideration items were administered on a daily basis. Across the 15 days that comprised the three internet measurement weeks, correlations between interpersonal commitment and support provided by the best friend were positive (mean correlation $=.55$; range $=.47$ to $.61 ; p$ 's $<.001)$. Interpersonal commitment was negatively correlated with negative interactions (mean correlation $=-.28$; range $=-.38$ to $-.18 ; p$ 's $<.01$ ). Interpersonal reconsideration was negatively correlated with support provided by the best friend (mean correlation $=-.50 ;$ range $=-.56$ to $-.38 ; p$ 's $<.001$ ), and positively correlated with negative interactions (mean correlation $=.46$; range $=.23$ to $.62 ; p$ 's $<.001$ ). Overall, these findings were in line with our expectations, and underscore the external validity of the single items tapping ideological and interpersonal commitment and reconsideration.

\section{Objective 2: Capturing the day-to-day dynamics of identity formation}

The three weeks of internet assessments each included five consecutive days of measurements for reconsideration and commitment in both domains. We assessed correlations in initial levels (i.e., Day 1 associations) and correlated change in reconsideration and commitment (i.e., Days 2, 3, 4, and 5 associations), whether levels of commitment affected levels of reconsideration one day later, and whether levels of reconsideration affected levels of commitment one day later. For this purpose, we used cross-lagged panel models with 5 consecutive daily measurements of commitment and reconsideration.

In these models, equivalent day-to-day cross-paths within weeks were constrained to be equal to one another (e.g., the path from reconsideration at Day 1 to commitment at Day 2 was constrained to be equal to the path from reconsideration at Day 2 to commitment at Day 3 , and so on), and correlated change coefficients were constrained to be equal throughout the week (e.g., the Day 2, Day 3, Day 4, and Day 5 associations of commitment and reconsideration were constrained to be equal to each other). In addition, equivalent paths and associations were constrained as equal across all three weeks. For example, the path from reconsideration at Day 1 to commitment at Day 2 was constrained to be equal in Weeks 1, 2, and 3. Both the model for ideological and the model for interpersonal identity had an excellent fit after these constraints were added. These model fits will be presented in the sections below. Because we tested a large number of paths in the structural equation models 
in with regard to objectives 2 and 3, we decided to focus only on the parameters that were significant at the .01 level.

Ideological Identity. The final model for day-to-day processes in ideological identity had an excellent fit $\left(\chi^{2}(71)=128.576(p<.001)\right.$, CFI $=.990 ;$ RMSEA $=.041(90 \%$ C.I. $=$ $.029-.052))$, that was just as good as the fit of the unconstrained model $(\Delta \mathrm{CFI}<.01$; Cheung $\&$ Rensvold, 2002). The final model is presented in Figure 8.1.

Figure 8.1 illustrates that not only the initial levels of commitment and reconsideration were negatively correlated; changes in levels of commitment and reconsideration were also negatively correlated. There were also significant cross-paths, indicating that higher levels of reconsideration on a specific day predicted lower levels of commitment on the next day. However, these paths were only weak and chi-square difference tests indicated that they did not differ significantly from the non-significant paths from commitment to reconsideration ( $p$ $>.05)$.

Interpersonal Identity. Like the model for ideological identity, the fit of the final, constrained, model for interpersonal identity was excellent $\left(\chi^{2}(71)=104.175(p=.006), \mathrm{CFI}\right.$ $=.996$; RMSEA $=.032(90 \%$ C.I. $=.018-.045))$, and just as good as the fit of the unconstrained model $(\triangle \mathrm{CFI}<.01)$. This model is presented in Figure 8.2.

For interpersonal identity, initial levels of commitment and reconsideration, and changes therein, were negatively correlated. In addition, higher levels of reconsideration on a specific day predicted lower levels of commitment on the next day and higher levels of commitment on one day predicted lower levels of reconsideration on the next day. Chi-square difference tests revealed that the paths from reconsideration to commitment were stronger than those from commitment to reconsideration $\left(\Delta \chi^{2}(1)=22.296 ; p<.001\right)$. 

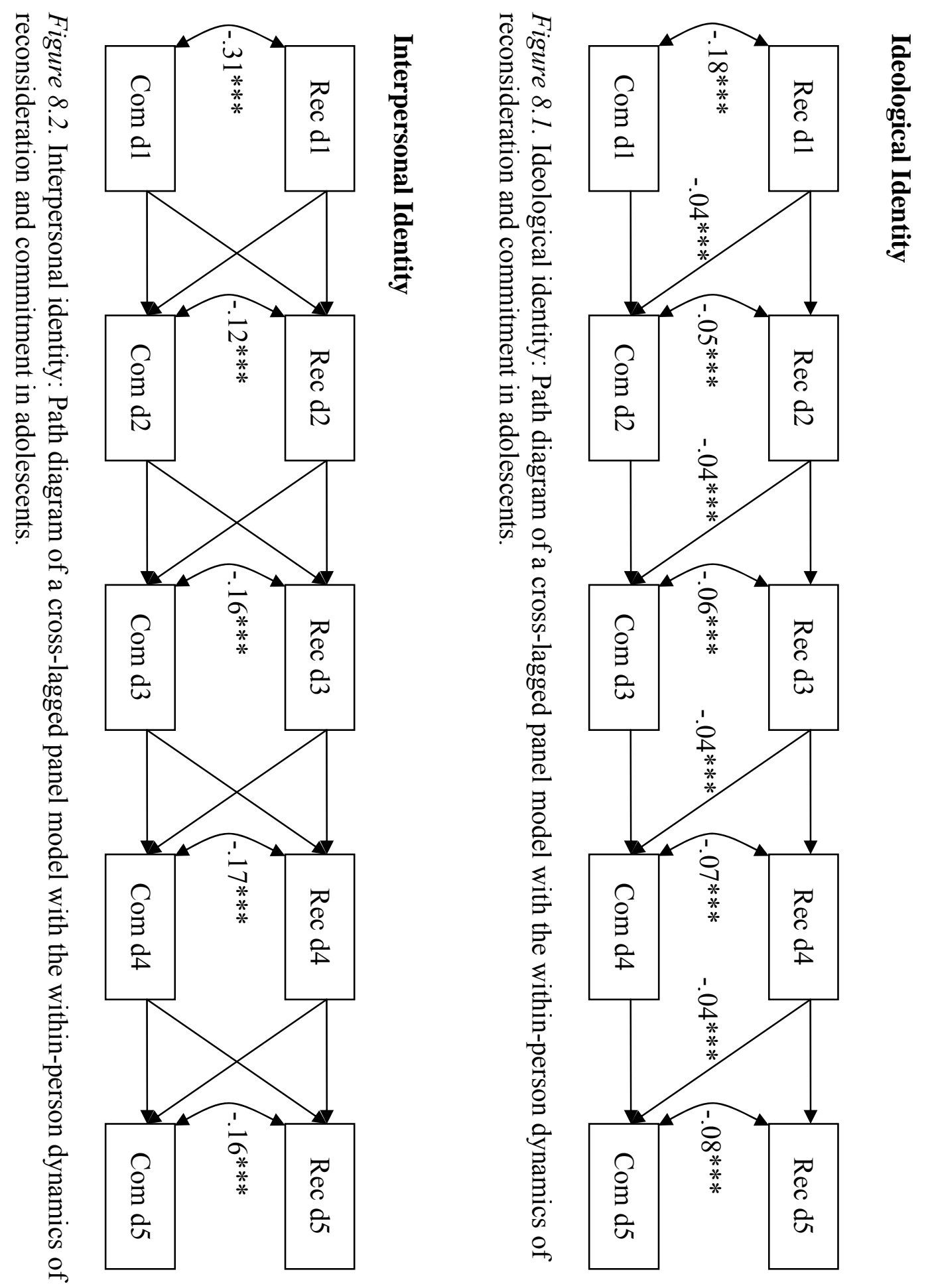


\section{Objective 3: Examining the Influences of Short-Term Fluctuations}

To measure fluctuations in commitment and reconsideration, we calculated intraperson standard deviations across the 5 days that comprised every internet week, for each dimension separately (see Kernis et al., 1989). Because 3 internet weeks were available, there were 3 measurements of across-week fluctuations available for each person and for both identity dimensions. Sample means and standard deviations of fluctuation scores, and acrossweek mean-levels of commitment and reconsideration are provided in Table 8.1.

As table 8.1 suggests, repeated measures ANOVA's revealed across-week mean levels of ideological and interpersonal commitment decreased $(F(2,1158)=15.236, p<.001$, partial $\eta^{2}=.026$; and $F(2,1158)=14.307, p<.001$, partial $\eta^{2}=.024$, respectively), whereas across-week mean levels of ideological and interpersonal reconsideration increased $(F(2$, $1158)=17.497, p<.001$, partial $\eta^{2}=.029$; and $F(2,1158)=31.671, p<.001$, partial $\eta^{2}=$ .052 , respectively).

However, our main focus was on fluctuations. Table 8.1 reveals that there were considerable across-week fluctuations in commitment and reconsideration in both identity domains. We assessed whether (a) fluctuations in commitment and reconsideration predicted one another over time (i.e., so-called cross-time paths), and whether (b) across-week fluctuations in reconsideration and commitment predicted the levels of commitment and reconsideration obtained at the annual assessment. Figure 8.3 displays the model we used to test these predictions.

As shown in this figure, all stability paths (e.g., fluctuations in commitment at Week 1 predicting fluctuations in commitment at Week 2) and concurrent associations (e.g., the association between fluctuations in commitment at Week 1 and fluctuations in reconsideration at Week 1) were included in the model. Equivalent paths and correlations (i.e., those parameters indicated with the same letters in Figure 8.3) were constrained to be equal across waves. The resulting models, which will be presented below, had an excellent fit to our data. 
Chapter 8

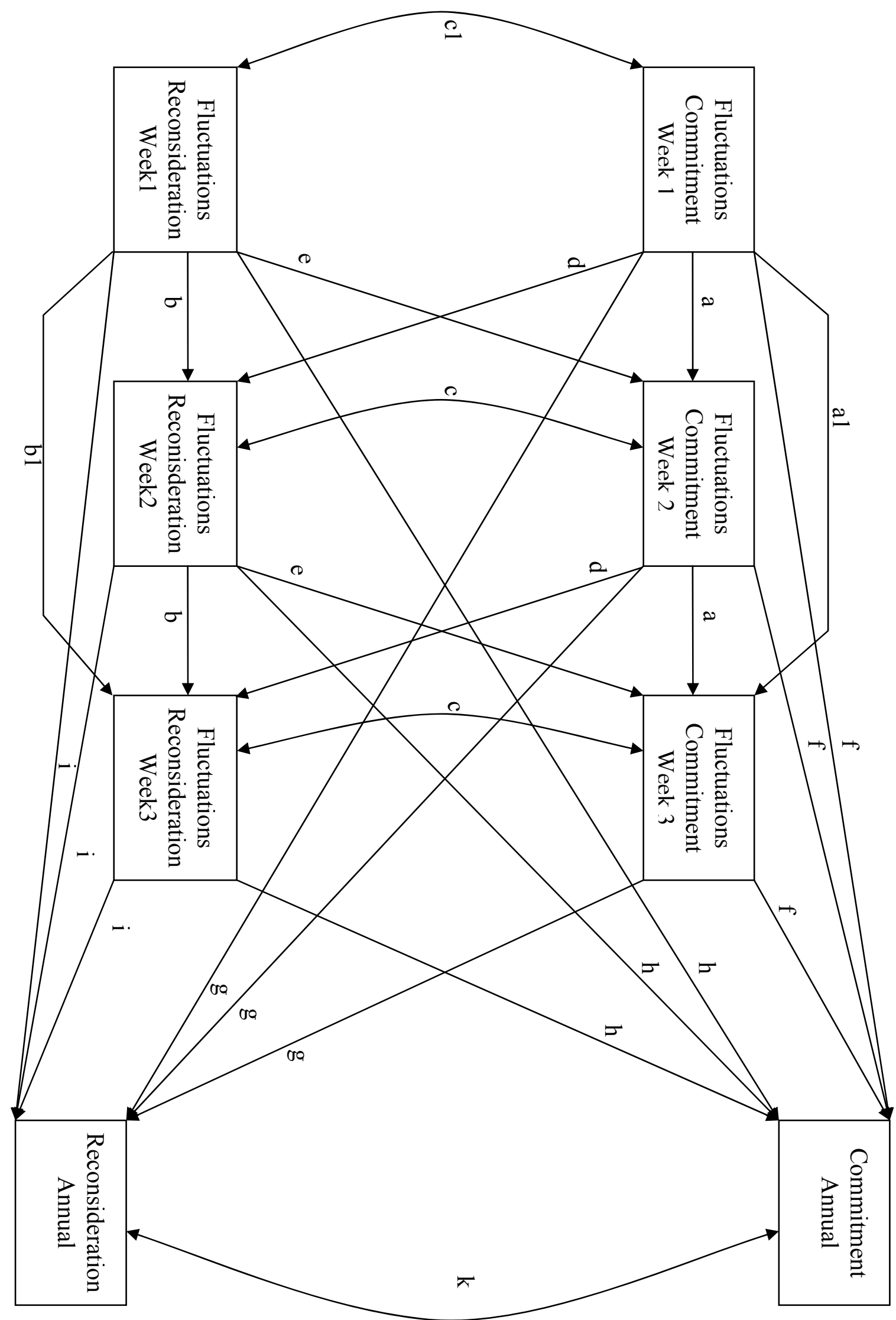


Figure 8.3 (previous page). Path diagram of a cross-lagged panel model with predictions of annual levels of reconsideration and commitment by across week fluctuations in these two processes. Equivalent paths and associations are signified by equivalent letters. Association ' $\mathrm{c} 1$ ' (between fluctuations in commitment and reconsideration) represents a correlation between initial levels, whereas the associations denoted by a 'c' represent associated change between the very same variables. Stability paths signified by ' $a$ ' and ' $b$ ' represent ordinary stability path between one measurement occasion and the subsequent one, those denoted by 'a1' and 'b1' represent extra stability paths from the first measurement occasion to the third measurement occasion.

Ideological Identity. The constrained model for ideological identity had an excellent fit $\left(\chi^{2}(16)=9.35\right.$ (n.s.), CFI $=1.00$; RMSEA $=.00(90 \%$ C.I. $\left.=.00-.02)\right)$, that was similar to the fit of the unconstrained model $(\Delta \mathrm{CFI}<.01)$. This model is displayed in Figure 8.4.

Figure 8.4 reveals that fluctuations in commitment and in reconsideration were moderately consistent across time. In addition, not only initial levels of fluctuations in commitment and reconsideration were positively related to one another (indicated by a significant association at Week 1), changes in fluctuations in commitment and in reconsideration (indicated by significant associations at Weeks 2 and 3) were also positively related to one another. Finally, more fluctuations in reconsideration predicted higher mean levels of reconsideration and lower mean levels of commitment at the annual measurement.

Interpersonal Identity. Similar to the model for ideological identity, the constrained model for interpersonal identity had an excellent fit $\left(\chi^{2}(16)=15.46\right.$ (n.s.), CFI $=1.00$; RMSEA $=.00(90 \%$ C.I. $=.00-.04))$. The fit of this constrained model was just as good as the fit of an unconstrained model $(\triangle \mathrm{CFI}<.01)$, and is shown in Figure 8.5.

Figure 8.5 indicates that fluctuations in commitment and in reconsideration were again moderately consistent across time. A significant correlation between Week 1 fluctuations in commitment and in reconsideration indicated that initial levels of fluctuations in commitment and fluctuations in reconsideration were positively associated. We also found evidence for correlated change of fluctuations in commitment and in reconsideration, indicated by positive correlations between these two variables at Weeks 2 and 3. Finally, more fluctuations in reconsideration predicted higher mean levels of reconsideration and lower levels of commitment at the annual assessment. 
Chapter 8

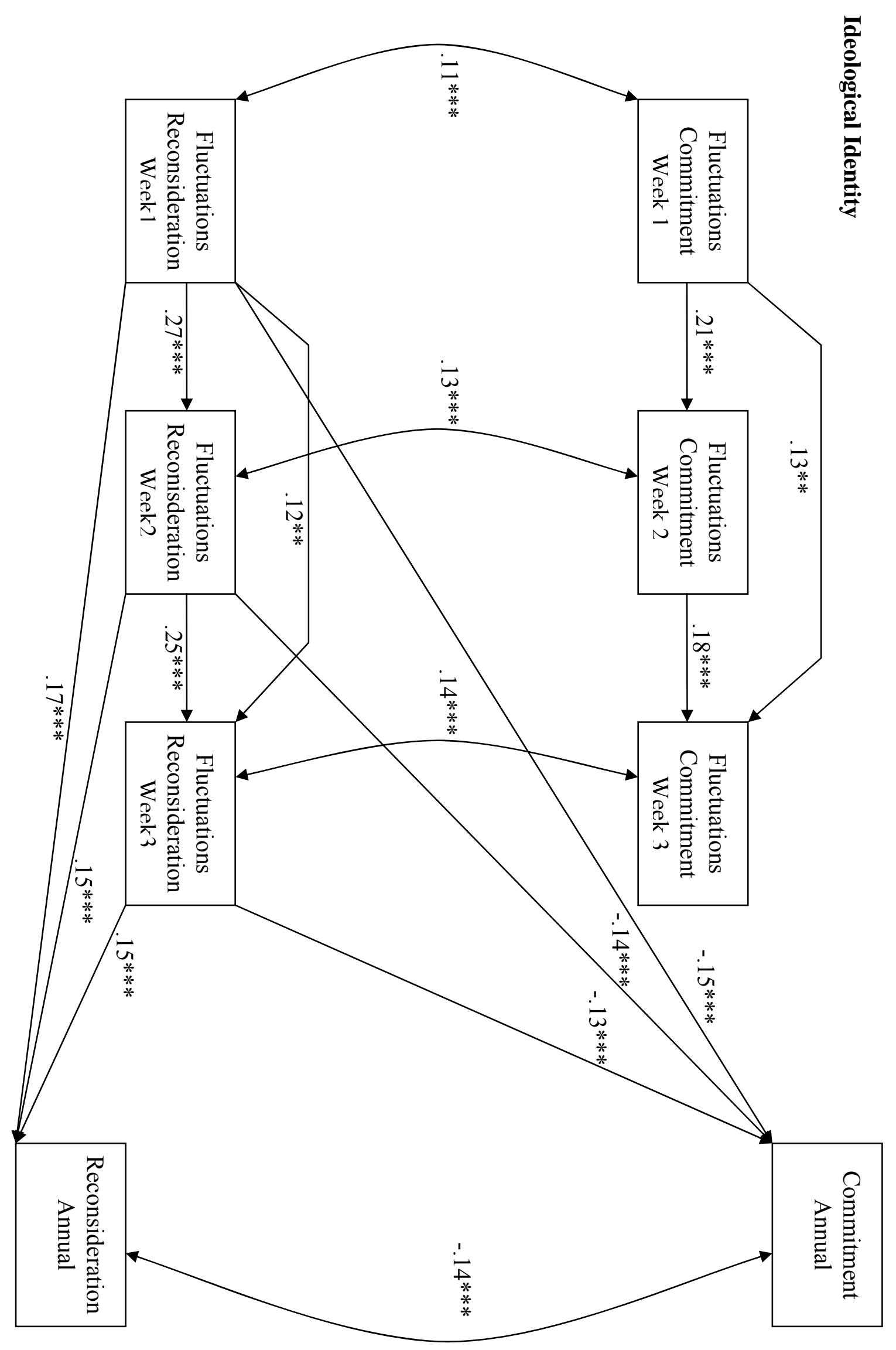




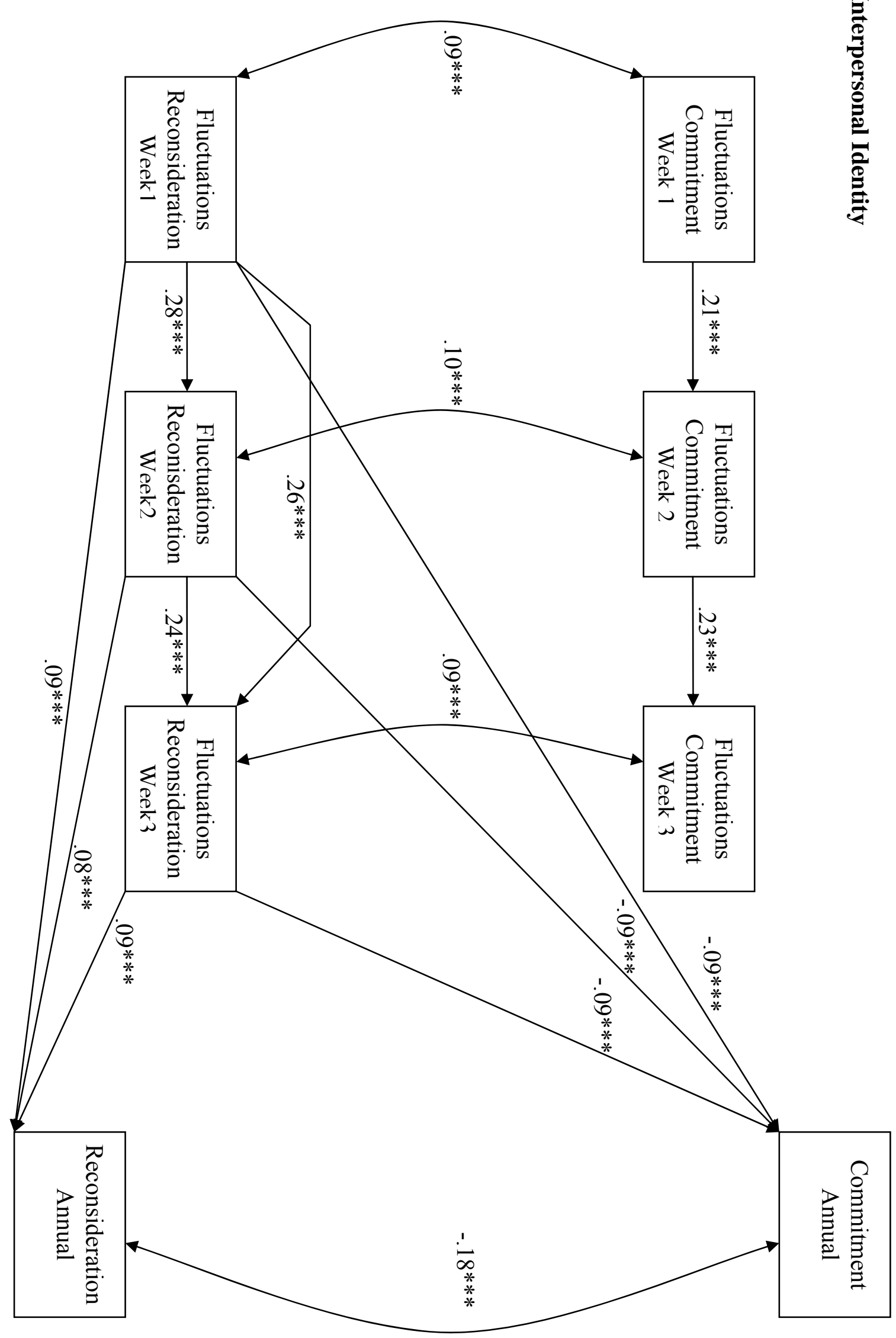


Figure 8.4 (previous page). Path diagram of a cross-lagged panel model with predictions of annual levels of reconsideration and commitment in the ideological domain as measured by across week fluctuations in these two processes.

Figure 8.5 (previous page). Path diagram of a cross-lagged panel model with predictions of annual levels of reconsideration and commitment in the interpersonal domain as measured by across week fluctuations in these two processes.

\subsection{Discussion}

The main purpose of the current study was to examine an important but relatively neglected aspect of identity formation, that is, the short-term or daily dynamics of identity formation (Lichtwarck-Aschoff et al., 2008; Schwartz, 2001). In the present research, we attempted to examine the day-to-day dynamics of two key identity formation processes: commitment and reconsideration. For that purpose, we (1) validated a new single-item measure to capture the day-to-day dynamics, (2) examined the day-to-day course of commitment and reconsideration, and (3) assessed how day-to-day fluctuations in commitment and reconsideration affected subsequent levels of these identity formation processes.

\section{Examining Short-Term Processes in Identity Formation: Validation of a Single-Item Measure}

Because the short-term dynamics of identity formation processes had not yet been examined in previous studies, we first needed to develop a measure to track such processes. With regard to reliability, Heise coefficients, which provide a reliable estimate of internal consistency based upon a test-retest reliability coefficients that separate true change from measurement error (Heise, 1969; Robins et al., 2001) were assessed. These analyses revealed that our short-term single-item scales for ideological and interpersonal commitment and reconsideration were all reliable. Validity was established by calculating the convergent (e.g., the correlation of the single item for ideological commitment with the multiple item scale for ideological commitment in the original version) and the discriminant validitity (e.g., the correlation of the single item for ideological commitment with the multiple item scale for ideological reconsideration in the original version). These analyses revealed that the correlation representing convergent validity was consistently higher than the correlation representing discriminant validity. 
As an additional check of the validity of our measures, we also examined the correlations between ideological commitment and reconsideration, and academic adjustment, and the correlations of interpersonal commitment and reconsideration with relationship quality with regard to the best friend. Our findings revealed that individuals with high levels of ideological commitment experience high levels of academic adjustment, whereas high levels of ideological reconsideration was related to low levels of academic adjustment. In the interpersonal domain, highly committed individuals experience a high quality of their relationship with their best friend, whereas individuals with high levels of reconsideration experience a low quality of the relationship with their best friend. These findings were in line with our expectations. Similar to previous studies (Crocetti, Rubini, \& Meeus, 2008) we found positive associations between commitment and adjustment, and negative associations between reconsideration and adjustment.

Overall, our results demonstrate that identity formation processes can be measured with single-item scales in a reliable and valid way. In addition to Robins et al. (2001), who concluded that their single-item self-esteem scale could provide a practical alternative for longer scales in contexts where time-constraints limit the number of items that could be administered, our findings suggest that single-item scales can also be a practical alternative for longer scales when measuring identity formation.

\section{The Day-to-Day Dynamics of Identity Formation}

After establishing the validity and reliability of our measure, we proceeded to examine the day-to-day dynamics of identity formation. With regard to ideological identity, we only found limited evidence for the presence of day-to-day processes. Although initial levels of commitment and reconsideration were substantially correlated with one another, changes in commitment were only weakly correlated with changes in reconsideration. In addition, levels of reconsideration on one day were predictive of levels of commitment on the next day, but these paths were only weak and did not differ significantly from the non-significant paths from commitment to reconsideration. Thus, there was only limited evidence for the presence of a certainty-uncertainty dynamic (i.e., commitment-reconsideration dynamic; Meeus et al., 2010) operating on a day-to-day basis in the ideological domain (i.e., education).

Evidence for a certainty-uncertainty dynamic in the interpersonal domain (i.e., relation with the best friend) was much stronger. Substantive correlations were found between initial levels and changes in commitment and reconsideration. Furthermore, commitment predicted reconsideration the next day, and reconsideration predicted commitment the next day. The 
predictive paths from reconsideration to commitment to reconsideration were much stronger than those in the inverse direction. Altogether, there was clear evidence for a commitmentreconsideration dynamic in the interpersonal domain.

The fact that the certainty-uncertainty dynamic seems to be much more vibrant in the interpersonal domain than in the ideological domain is in line with Meeus et al.'s (1999) distinction between open and closed domains of identity formation. Meeus et al. (1999) proposed that open domains, such as friendships, would be much more open to change than closed domains, such as education. The main reason for this is that adolescents may not find it useful to explore commitments in areas on which they can exert relatively little influence. As an early adolescent has to follow some sort of education while there are a limited number of alternatives available, exploration might become less activated in some adolescents. This could be the reason why there is only weak evidence for a certainty-uncertainty dynamic in the ideological domain of education. With regard to friendships, much more options are available. Adolescents have been shown to be changing frequently from one best friend to another (Branje et al., 2007; Connolly et al., 2000; Degirmencioglu et al., 1998), and therefore seem to be engaged in an active exploration process with regard to choosing friends that suit their needs best. Thus, loose ties and a tendency to change from one best friend to another may be socially undesirable in other phases in life, but seem normative for adolescents.

Across domains, paths from reconsideration to commitment are stronger than those in the inverse direction. Thus, it seems to be reconsideration that drives the re-shaping of commitments. Considering that the current study samples early adolescents and that levels of commitment decrease while levels of reconsideration increase, we are quite likely to have captured the initial stages of identity formation in which adolescents start to reconsider and refute their childhood identifications (Erikson, 1950, 1968). Our findings not only suggest that early adolescents' commitments are gradually weakened, we also uncovered that these changes in the strength of commitments are driven by increases in reconsideration. That is, adolescents first start to compare their commitments to possible alternatives (i.e., reconsideration) before the strength of current commitments starts to weaken. As such, our results provide an important insight into how early adolescent identity formation works. Because our results suggest that commitments are reshaped on a day-to-day basis, identity indeed seems to function as a dynamic and self-organizing system (Kunnen et al., 2001). 


\section{Relating Short-Term Processes of Identity Formation to Long-Term Processes}

Day-to-day processes in identity formation are interesting in themselves, but it is also important to examine how these short-term fluctuations influence identity formation in the long run. As such, we examined the influence of short-term day-to-day fluctuations in commitment and reconsideration on general levels of these two processes. For both ideological and interpersonal identity, we found that fluctuations in commitment and reconsideration were correlated with one another. What is more, fluctuations in reconsideration predicted levels of both commitment and reconsideration in the ideological and interpersonal domain. Thus, adolescents who occasionally reconsider their commitments exhibit higher levels of reconsideration and lower levels of commitment in general. Therefore, an inconsistent attitude towards reconsideration predicts a weaker identity. As such, fluctuations in reconsideration could be part of the psychological moratorium described by Marcia (1966). Adolescents in a state of moratorium are struggling to form self-defined commitments. They still feel commitment towards their parents' teachings, but are "attempting a compromise among them, society's demands, and his own capabilities" (Marcia, 1966; p. 552). Marcia stated that this task sometimes seems irresolvable to them. This is possibly reflected in adolescents reconsidering their commitments on some days (i.e., when they feel they can come to a compromise between their own, their parent's, and society's demands), while they accept their current commitments on other days because they feel like they cannot resolve the tangible task that adolescent identity formation is. Such a moratorium-like state implies that an adolescent's identity is temporarily weak, but might be necessary for establishing a robust and self-defined identity on the long term (Erikson, 1950, 1968; Marcia, 1966).

Overall, our findings fit into Erikson's $(1950 ; 1968)$ conceptualization of identity formation as a quest for sameness and continuity. Experiencing sameness and continuity in the way one deals with an identity (depicted by stable levels of reconsideration) is predictive of a more robust identity in general (indicated by higher levels of commitment and lower levels of reconsideration).

\section{Strengths, Limitations, and Future Directions}

Altogether, the current study is characterized by several strengths. The most important strength of the current study is its focus on short-term processes of identity formation, which had been relatively neglected until now (Grotevant, 1987; Kunnen et al., 2001; LichtwarckAschoff et al., 2008). Second, we used five measurement occasions in 3 weeks in a single 
year. Because we found comparable day-to-day processes across days and across weeks, the likelihood that our findings occurred by chance is minimal. Therefore, our findings should at least partially reflect the way certainty-uncertainty dynamics of identity formation operate for early adolescents. Third, we followed recommendations by Lichtwarck-Aschoff et al. (2008), by relating micro-level processes (i.e., day-to-day fluctuations) to macro-level processes of identity formation (i.e., mean levels of commitment and reconsideration reflecting general attitudes towards identity formation). It turned out that micro-level processes are indeed related to macro-level processes.

Despite these strengths, several limitations need to be recognized. The exclusive focus on early adolescents can be considered as a first limitation. It remains unclear how short-term processes operate in late adolescence. Theorists (e.g., Bosma \& Kunnen, 2008) have argued, and empirical studies (e.g., Klimstra et al., 2009) have shown, that more abstract selfreflective identity processes, such as in-depth exploration (i.e., reflection on current commitments, searching additional information on these commitments, and discussing them with relevant others) start to operate in late adolescence. The short-term dynamics of these identity processes should be studied in future research.

The focus of the current study was limited to identity processes and their associations with friendship quality and academic adjustment. Other variables that have been shown to be related to identity, such as personality (Luyckx, Soenens, \& Goossens, 2006), separationindividuation (Kroger \& Haslett, 1988; Meeus et al., 2005), and internalizing and externalizing problems (e.g., Crocetti et al., 2008; Luyckx, Goossens, Soenens, \& Beyers, 2006), should be related to short-term fluctuations in identity formation. Such studies could be informative on whether short-term changes in identity formation have an immediate impact on adolescent functioning and might even unravel what it is that triggers these short-term changes. Future studies could also examine how short-term fluctuations in identity affect general psychosocial functioning.

A possible third limitation is that we only considered adolescents themselves. Adolescents do, of course, not live in a social vacuum. Instead, they usually live together with parents, siblings, and peers. The importance of parents and peers with regard to identity formation has already been demonstrated. In a laboratory setting, Kerpelman and colleagues (Kerpelman, \& Lamke, 1997; Kerpelman, Pittman, \& Lamke, 1997; Kerpelman, \& Pittman, 2001) demonstrated that identity processes were affected by interpersonal communication between adolescents and their friends. Grotevant and Cooper (1985) revealed that observed real-time parent-child interactions triggered identity exploration. However, these studies did 
Short-Term Fluctuations in Identity

not include multiple measurement occasions. To better examine how parents, siblings, and friends influence adolescent identity formation, we would encourage researchers to examine these influences on a day-to-day basis.

Despite these potential limitations, the present study provides a substantial contribution to research on identity formation. Our findings indicate that identity formation processes operate on a day-to-day basis, and that these day-to-day fluctuations have a substantive impact on subsequent levels of two key identity dimensions (i.e., commitment and reconsideration). Altogether, the current study provides an important first step in uncovering the day-to-day dynamics of identity formation. 


\section{Chapter 9}

Identity Formation in Juvenile Delinquents, Clinically Referred Youth, and Adolescents from the General Population ${ }^{9}$

${ }^{9}$ Klimstra, T. A., Crocetti, E., Hale, W. W., Kolman, A. I. M, Fortanier, E. L., \& Meeus, W. H. J. (2009). Identity formation in juvenile delinquents, clinically referred youth, and adolescents from the general population. Manuscript submitted for publication. 


\begin{abstract}
The purpose of the present study was to assess identity formation in juvenile delinquent boys. For this reason, these youth were compared to clinically referred boys and boys drawn from the general population. Our results suggest that both ideological (i.e., education) and interpersonal (i.e., relation with the best friend) identity formation were problematic for juvenile delinquents, as many of them seemed to have trouble with the achievement of stable commitments. As problems with identity formation could be an underlying cause of problem behavior, our findings suggest that identity formation among juvenile delinquents deserves more attention from both researchers and clinicians.
\end{abstract}




\subsection{Introduction}

Adolescence has continuously been described as a period of "storm-and-stress" (Arnett, 1999). Although this storm-and-stress remains limited to conflicts with parents, temporary mood disruptions, and minor delinquent acts in most adolescents (Arnett, 1999), problems escalate among a minority of youth. Some adolescents are engaged in serious delinquent acts and end up in juvenile detention centres. In the Netherlands, approximately seven thousand adolescents live in such institutions (Dutch Department of Justice, 2008). If these juvenile delinquents receive psychotherapy, the therapy is usually aimed at increasing their moral reasoning ability, a developmental task that many juvenile delinquents have not fully mastered (e.g., Gibbs, Potter, Barriga, \& Liau, 1996). However, limited attention has been devoted to another key developmental task that could be an underlying cause of the problems faced by these troubled youth: the development of a firm personal identity (Erikson, 1950). Since the formation of an identity requires exploration, this key developmental task might be particularly hard to accomplish in highly structured settings like juvenile detention centres. Unfortunately, the extent to which these troubled youth face problems with identity formation is yet unclear. Therefore, the goal of this study is to examine whether identity formation is problematic for juvenile delinquents. In order to accomplish this goal we will compare identity formation in juvenile delinquents and two control groups: clinically referred youth and adolescents from the general population.

\section{Research on Identity Formation: A Short Overview}

The formation of a stable identity is considered to be the most important developmental task for an adolescent (Erikson, 1950). An individual's identity can be classified along a continuum from identity achievement (reflected by the presence of a stable set of ideals and morals) to role confusion (the inability to establish a useful set of ideals; Schwartz, 2001).

In the field of research on adolescent identity formation, the identity status paradigm (Marcia, 1966) is the dominant approach. Marcia distinguished two important factors in identity formation: commitment (selection of developmental alternatives and engagement in relevant activities towards the implementation of these choices) and exploration (exploring various developmental alternatives in different identity-defining domains). Based on levels of commitment and exploration, four identity statuses can be distinguished (Marcia, 1966): diffusion (little exploration, little commitment), foreclosure (strong commitments, but 
alternatives have not been explored), moratorium (intense exploration, but no strong commitments yet), and achievement (strong commitments, formed after a period of extensive exploration).

Marcia's (1966) identity statuses are based on two processes of identity formation (i.e., exploration and commitment). However, several researchers (Balistreri, BuschRossnagel, \& Geisinger, 1995; Luyckx, Goossens, Soenens, \& Beyers, 2006) have argued that especially exploration is not a unidimensional construct. As an alternative, these and other scholars have proposed several extensions of the identity status paradigm throughout the last two decades. One of the most recent extensions of Marcia's status paradigm is the threedimension model proposed by Meeus (Crocetti, Rubini, \& Meeus, 2008b). In this threedimension model, processes of commitment (choices made in identity relevant domains and the extent to which individuals identify themselves with these choices), in-depth exploration (exploring the merits of one's current commitments), and reconsideration of commitment (questioning current commitments, and searching for possible alternatives) are distinguished. The three-dimension model of identity formation has been shown to provide a valid and reliable estimate of identity processes in early, middle and late adolescents, boys and girls, and ethnic majorities and minorities (Crocetti et al., 2008b). Even though the dimensions of identity formation described by Meeus and colleagues (Crocetti et al., 2008b) are slightly different from those described by Marcia (1966), Crocetti, Rubini, Luyckx, and Meeus (2008a) were able to replicate Marcia's four identity statuses: achievement (high commitment, high exploration, and low reconsideration), foreclosure (moderate commitment, low exploration, and low reconsideration), moratorium (low commitment, low exploration, and high reconsideration), and diffusion (low commitment, low exploration, and low reconsideration). However, Crocetti et al. (2008a) found one additional moratorium status besides the one originally described by Marcia. This status, labelled searching moratorium, captures the positive side of moratorium: it comprises adolescents who are highly committed but nevertheless keep on exploring alternatives, and thus are revising their identities. In a recent longitudinal study spanning the entire period from early to late adolescence, Meeus, van de Schoot, Keijsers, Schwartz, and Branje (2010) showed that searching moratorium is a transitory status which only exists in early to middle adolescence, but disappears in late adolescence. The prevalence of the classical moratorium status also decreases in late adolescence, but there is a substantial subgroup which remains in this type of moratorium. Thus, the classical moratorium status seems to be a more enduring asset, whereas searching moratorium appears to be a passing state. 
Throughout the years, linkages between identity statuses and psychosocial adjustment (i.e., internalizing and externalizing problem behavior) have been uncovered and replicated (e.g., Crocetti et al., 2008a; Luyckx et al., 2008; Marcia, 1966; Meeus, Iedema, Helsen, \& Vollebergh, 1999). An overview by Meeus et al. (1999), mainly focused on associations of identity with internalizing problems (e.g., anxiety, depression), revealed that individuals in identity statuses characterized by strong commitments (i.e., those in foreclosure and achievement statuses) had lower levels of problem behavior symptoms when compared to adolescents who were high in exploration and low in commitment (i.e., those in the classical moratorium status). Subsequent studies (e.g., Crocetti et al., 2008a; Luyckx et al., 2008) on identity statuses and adjustment obtained similar results. In addition, Crocetti et al. (2008a) found that adolescents in the classical moratorium status were characterized by higher levels of externalizing problem behavior symptoms than adolescents in other statuses. Adolescents in the searching moratorium status were found to have lower scores on both internalizing and externalizing problem behavior symptoms when compared to adolescents in a classical moratorium status, but they did have somewhat higher levels of depressive symptoms when compared to achievers, foreclosures, and diffused adolescents. These findings suggest that searching moratorium is only related to self-doubt (i.e., depressive symptoms), whereas a classical moratorium is indicative of a simple lack of commitment resulting in both internalizing and externalizing problem behavior symptoms.

In addition to the clear linkages between identity formation and psychosocial adjustment, Luyckx, Goossens, Soenens, Beyers, and Vansteenkiste (2005) also uncovered strong linkages with social and academic adjustment. Again, individuals in achievement and foreclosure statuses turned out to be the best-adjusted. Overall, these findings suggest that identity statuses are related to social, academic, and psychosocial adjustment, with individuals in achievement and foreclosure statuses exhibiting higher levels of adjustment than those in diffusion and moratorium statuses.

Although linkages between identity statuses and adjustment have been clearly documented, these findings have been obtained in generally well-adjusted population samples. Moreover, all the mentioned studies employed global identity measures examining identity across several domains, instead of focusing on specific domains. Erikson (1950) initially focused on identity formation as a process that mainly takes place in ideological domains (e.g., politics, education), but Grotevant, Thorbecke, and Meyer (1982; Grotevant \& Adams, 1984) extended this focus into interpersonal domains (e.g., friendships, romantic relations). Thus, there are two main types of identity domains (i.e., ideological and 
interpersonal domains), which can be further subdivided in more specific domains such as education (i.e., an ideological domain) and friendships (i.e., an interpersonal domain). An example of the meaning of identity statuses in these two different domains would be that an individual classified as "achieved" for educational identity is highly committed to his study and often thinking about possible job opportunities after graduation. An individual who is highly committed to current friends and often thinks about what these friendships mean to him/her would be in the "achievement" status in the friendship domain

\section{The current study}

In the current study, we aim to investigate ideological (i.e., educational) and interpersonal (i.e., relational) identity formation in juvenile delinquents (i.e., adolescents living in a penitentiary youth institution because they exhibited norm-breaking behavior). Research on identity formation has examined several other domains, such as gender identity (for an overview, see Ruble, Martin, \& Berenbaum, 2006) and ethnic identity (for an overview, see Phinney \& Ong, 2007). However, we chose to follow Erikson's (1950) early focus on identity formation as a process that mainly takes place in ideological domains (i.e., education), and the extension of that focus by Grotevant et al. (1982) into interpersonal domains (i.e., friendships). The focus of the current study is on these ideological and interpersonal domains, because an adolescent actually has something to choose and can identify him or herself with that choice, in contrast to gender and ethnic identity in which an adolescent can just identify him/herself with a more static personal characteristic.

To examine identity formation in juvenile delinquents, we will compare these youth with two comparison samples: adolescents drawn from the general population and clinically referred youth residing in a residential youth institution because they were facing severe emotional distress. Employing this three-group design allows us to examine whether all youth facing severe psychological problems (i.e., either norm-breaking behavior, or emotional distress) have troubles with identity formation, or whether a problematic identity formation applies specifically to youth engaged in norm-breaking behaviors (i.e., juvenile delinquents). Both the juvenile delinquents and the clinically referred sample are institutionalized, whereas the adolescents drawn from the general population are living with their parents. As such, if juvenile delinquents would be different with regard to their identity when compared to clinically referred youth, these differences could not be fully ascribed to the effects of institutionalization. 
Because juvenile delinquents display a deficient sense of moral reasoning (e.g., Gibbs et al., 1996), we expect them to exhibit a lacking sense of commitment towards societal institutions (i.e., education) and relevant others (i.e., friends). As such, juvenile delinquents are likely to exhibit a classical moratorium or a diffusion status of interpersonal and ideological identity, and unlikely to exhibit the more mature statuses achievement, foreclosure, and searching moratorium statuses. Shedding light on these issues could provide valuable indications for interventions aimed at promoting a more healthy development among juvenile delinquents.

\subsection{Method}

\section{Participants}

In the current study, three adolescent samples were distinguished: a sample of juvenile delinquents, a sample of clinically referred youth, and a sample of adolescents from the general population. All participants were boys. The juvenile delinquent sample comprised 30 adolescent boys residing in a penitentiary youth institution in the Netherlands. All were referred to the institution by a Dutch court. Our sample of clinically referred youth included 21 adolescent boys residing in a residential youth institution in the Netherlands. These youth required institutional care because their parents were no longer able to provide adequate care, because of severe behavioral problems of the adolescent themselves or because of their fathers' and/or mothers' lack of adequate parenting skills. They participated in a psychological foster care program, aimed at teaching them the psychological and socialization skills they would eventually need to earn an independent living. There were no inclusion criteria for juvenile delinquents and the clinically referred youth. The sample derived from the general population, matched to the delinquent sample with regard to background characteristics (e.g., age, educational background of the parents), consisted of 30 adolescent boys derived from a larger population sample that participated in a five-wave longitudinal research project on CONflict And Management Of RElationships (CONAMORE; Meeus et al., 2006). All adolescents (including juvenile delinquents and clinically referred youth) received education. The three samples were compared on age, ethnicity, and educational background of the parents.

The delinquent sample was somewhat older on average (16.83 years of age; $S D=$ $2.00)$ than the clinical sample (15.52 years of age; $S D=1.17 ; p<.05)$. The mean age of the comparison sample $(16.63$ years; $S D=1.87)$ was not significantly different from the mean 
age of the clinical and the delinquent sample. For ethnicity, a distinction was made among adolescents that identified themselves as being Dutch, and those that identified themselves as being non-Dutch (e.g., Surinamese, Moroccan, Turkish). The ethnic composition of the clinical (90.5\% Dutch) and delinquent sample (96.7\% Dutch) was equal, but chi-square tests indicated that the proportion of Dutch adolescents was significantly lower in the comparison sample $(73.3 \%)$ than in the delinquent sample $(p=.01)$. However, additional analyses showed that excluding ethnic minorities from our samples had no substantive impact on outcomes. The parental educational level was higher among clinically referred youth $(100 \%$ of the fathers and $92.3 \%$ of the mothers had completed high school) than among juvenile delinquents ( $75 \%$ of the fathers and $56.5 \%$ of the mothers finished high school). Adolescents from the general population $(87.5 \%$ of the fathers and $69 \%$ of the mothers finished high school) did not differ from juvenile delinquents and clinically referred youth with regard to parental educational level.

\section{Procedure}

In all three samples, participants and their parents received an invitation letter, describing the research project and goals, and explaining the possibility to decline from participation. After a detailed instruction, all participants signed an informed consent form. For the clinical and delinquent sample, consent was also obtained from the participating institutions. The clinical and delinquent samples completed questionnaires in their own institution, whereas the adolescents from the general population completed the questionnaires at their own high school. Confidentiality of responses was guaranteed. Verbal and written instructions were offered.

\section{Measures}

Identity. Identity was assessed with the Utrecht-Management of Identity Commitments Scale (U-MICS), a self-report measure designed by Meeus (Crocetti et al., 2008b; Meeus et al., 2010) based on the Utrecht-Groningen Identity Development Scale (Meeus, 1996). The UMICS consists of 13 items with a response scale ranging from 1 (completely untrue) to 5 (completely true). The same items can be filled out to assess identity dimensions in different domains. In the current study, we focused on two domains that are salient for every adolescent: education and friendships. Hence, each item was presented once for an ideological domain (i.e., education) and once for an interpersonal domain (i.e., friendships) for a total of 26 items. Sample items are: "My education/best friend gives me certainty in life" 
(ideological/interpersonal commitment; 5 items per domain), "I think a lot about my education/best friend" (ideological/interpersonal in-depth exploration; 5 items per domain), and "I often think it would be better to try and find different education/a different best friend" (ideological/interpersonal reconsideration; 3 items per domain). The three-factor structure of U-MICS has been confirmed for both boys and girls, both early and middle adolescents, and both Dutch and ethnic minority youths (Crocetti et al., 2008b). Cronbach's alphas for ideological identity were $.90, .94, .94$ for commitment; .81, .80, .90 for in-depth exploration; and $.88, .90, .72$ for reconsideration of commitment in the delinquent sample, clinical sample, and adolescents from the general population, respectively. For interpersonal identity, Cronbach's alphas were $.86, .89, .94$ for commitment; $.60, .74, .88$ for in-depth exploration; and $.84, .83, .86$ for reconsideration of commitment in the delinquent sample, clinical sample, and adolescents from the general population, respectively.

\section{$9.3 \quad$ Results}

Means and standard deviations of the clinical sample, delinquent sample, and adolescents from the general population for commitment, in-depth exploration, and reconsideration of commitment are displayed in Table 9.1. All three dimensions were examined in an ideological (i.e., education) and an interpersonal identity domain (i.e., relation with the best friend).

\section{Identity Processes}

Ideological Identity. For ideological identity, a multivariate analysis of covariance (MANCOVA) in which we controlled for the adolescents age and educational level of father and mother, revealed that there were overall differences between the three samples $(F(6,148)$ $=5.21 ; p<.001)$. Univariate tests indicated that these differences were significant for commitment $(F(2,75)=15.94 ; p<.001)$ and in-depth exploration $(F(2,75)=8.65 ; p<.001)$. Bonferroni tests for pair-wise comparisons between all possible pairs of groups (i.e., clinical sample - delinquent sample, clinical sample - comparison sample, and delinquent sample comparison sample) revealed that delinquent adolescents significantly differed from adolescents drawn from the general population, as they displayed significantly lower levels of commitment and in-depth exploration (see Table 9.1). Delinquent adolescents were also significantly less committed than clinically referred youth. 
Table 9.1

Means and Standard Deviations of Clinical, Delinquent, and Comparison Samples on Identity Dimensions

\begin{tabular}{llcc}
\hline & $\begin{array}{c}\text { Clinical Sample } \\
(n=21)\end{array}$ & $\begin{array}{c}\text { Delinquent Sample }(n \\
=30)\end{array}$ & $\begin{array}{c}\text { Comparison Sample }(n \\
=30)\end{array}$ \\
\hline $\begin{array}{c}\text { School Identity } \\
\text { Commitment } \\
\text { In-depth Exploration }\end{array}$ & $3.54^{\mathrm{a}}(1.07)$ & $2.35^{\mathrm{b}}(0.96)$ & $3.79^{\mathrm{a}}(0.88)$ \\
$\begin{array}{c}\text { Reconsideration } \\
\text { Relational Identity }\end{array}$ & $2.63^{\mathrm{a}}(1.29)$ & $3.55^{\mathrm{b}}(0.95)$ & $3.57^{\mathrm{a}}(0.87)$ \\
Commitment & & & $2.74^{\mathrm{a}}(1.01)$ \\
$\quad$ In-depth Exploration & $3.19^{\mathrm{a}, \mathrm{b}}(0.92)$ & $2.63^{\mathrm{a}}(0.93)$ & $3.54^{\mathrm{b}}(0.89)$ \\
Reconsideration & $3.16^{\mathrm{a}}(0.78)$ & $2.85^{\mathrm{a}}(0.71)$ & $3.30^{\mathrm{a}}(0.89)$ \\
& $1.81^{\mathrm{a}}(0.96)$ & $4.06^{\mathrm{b}}(1.04)$ & $2.49^{\mathrm{a}}(1.13)$ \\
\hline
\end{tabular}

Note. Different superscripts represent significant mean-levels differences between samples $(p<.05)$. Samples with different superscripts across rows differ from one another with regard to an identity dimension (e.g., commitment). Differences were assessed by the means of pair-wise comparisons (i.e., clinical sample delinquent sample, clinical sample - comparison sample, and delinquent sample - comparison sample) with Bonferroni tests.

Interpersonal Identity. A multivariate analysis of covariance (MANCOVA), in which we controlled for age, and educational level of father and mother, revealed that there were overall differences between the three samples with regard to interpersonal identity processes $(F(6,148)=9.19 ; p<.001)$. Univariate tests indicated that these differences were only significant for commitment $(F(2,75)=7.02 ; p=.002)$ and reconsideration $(F(2,75)=27.36$; $p<.001)$. In a subsequent step, post-hoc Bonferroni tests revealed that delinquent adolescents were clearly differentiated from adolescents from the general population, since they displayed significantly lower levels of commitment and higher levels of reconsideration (see Table 9.1). Delinquent adolescents also displayed significantly higher levels of reconsideration when compared to clinically referred youth.

\section{Identity Statuses}

Ideological Identity. To extract identity statuses for the three samples, we used a twostep cluster analysis procedure (Gore, 2000) on the combined sample. Before conducting cluster-analyses, scores on all three ideological identity dimensions were standardized. In the first step, a hierarchical cluster analysis was conducted using Ward's method based on squared Euclidian distances. The cluster centers obtained in the first step were then used as 
starting values in the second step: the $k$-means cluster analyses. Different cluster solutions were compared. On the basis of three criteria (i.e., theoretical meaningfulness, parsimony, and explanatory power), we finally retained a five-cluster solution (see Figure 9.1) that strongly resembles the solution found in a large Dutch community sample (Crocetti et al., 2008a). The same cluster solution was replicated in all three samples, separately. Furthermore, the degree of correspondence, calculated by means of Cohen's kappa coefficient, between the cluster solution obtained in the entire sample (including all three samples) and cluster solutions obtained in separate samples was acceptable, with values ranging from .82 to .95 , supporting the replicability of the five-cluster solution.

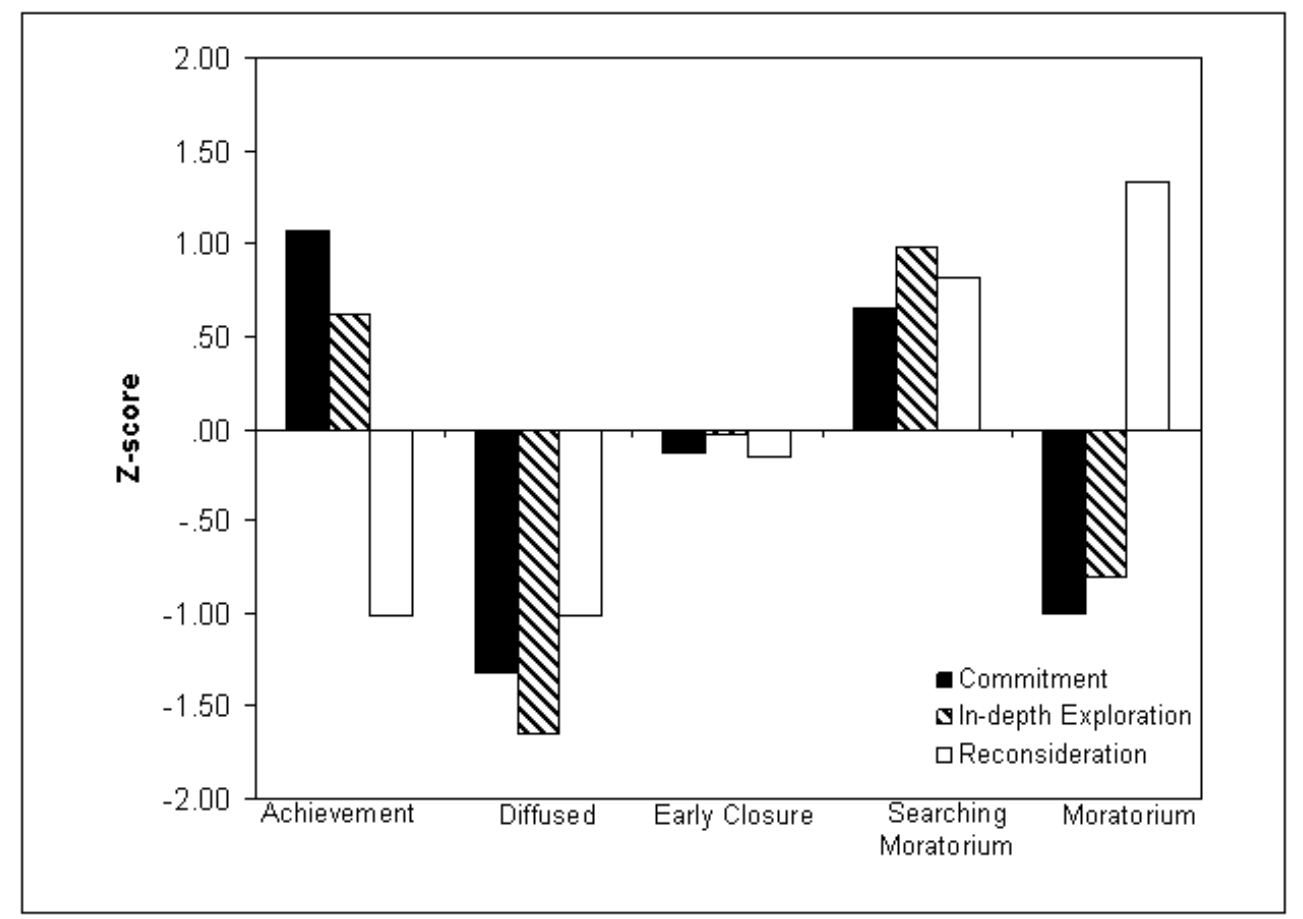

Figure 9.1

Final cluster-solution in the combined sample for ideological identity. Standardized means for commitment, in-depth exploration, and reconsideration.

The five clusters were labelled early closure (medium scores on all three identity dimensions), achievement (high on commitment and in-depth exploration, low on reconsideration), searching moratorium (high on all three identity dimensions), diffusion (low on all three identity dimensions), and moratorium (low on commitment and in-depth exploration, high on reconsideration). The distribution of the participants from the three samples across the five clusters was very heterogeneous (see Table 9.2). 
Table 9.2

Distribution of Adolescents from Clinical, Delinquent, and Comparison Samples across Five Ideological Identity Clusters

\begin{tabular}{lccc}
\hline & $\begin{array}{c}\text { Clinical Sample } \\
(n=21)\end{array}$ & $\begin{array}{c}\text { Delinquent Sample } \\
(n=30)\end{array}$ & $\begin{array}{c}\text { Comparison Sample } \\
(n=30)\end{array}$ \\
\hline Early Closure & $6(28.6 \%)$ & $9(30.0 \%)$ & $11(36.7 \%)$ \\
Achievement & $7(33.3 \%)^{\mathrm{a}}$ & $1(3.3 \%)^{\mathrm{b}}$ & $9(30.0 \%)^{\mathrm{a}}$ \\
Searching Moratorium & $4(19.0 \%)$ & $3(10.0 \%)$ & $9(30.0 \%)$ \\
Diffused & $2(9.5 \%)^{\mathrm{a}, \mathrm{b}}$ & $6(20.0 \%)^{\mathrm{a}}$ & $1(3.3 \%)^{\mathrm{b}}$ \\
Moratorium & $2(9.5 \%)^{\mathrm{a}}$ & $11(36.7 \%)^{\mathrm{b}}$ & $0(0.0 \%)^{\mathrm{a}}$ \\
\hline
\end{tabular}

Note. Percentages represent the percentage of adolescents within a sample that is assigned to a cluster. Hence, percentages within a column add up to $100 \%$. When samples have different superscripts across rows, the proportion of individuals that were classified in a certain status was significantly different $(p<.05)$. Differences were assessed by the means of pair-wise comparisons (i.e., clinical sample - delinquent sample, clinical sample - comparison sample, and delinquent sample - comparison sample) with Fisher's Exact Tests, using Monte Carlo approximation.

Fisher's Exact Test (using Monte Carlo approximation to deal with issues related to our small sample sizes) indicated that there were significant overall differences in prevalence of the clusters between the three samples in this study $(p<.001)$. Post-hoc pair-wise comparisons between all possible pairs of samples (i.e., clinical sample - delinquent sample, clinical sample - comparison sample, and delinquent sample - comparison sample) revealed that delinquents were more likely to be in the moratorium cluster, and less likely to be in the achievement cluster when compared to clinically referred youth and adolescents from the general population. In addition, delinquents were also more likely to be classified in the diffused cluster when compared to adolescents from the general population. Clinically referred youth and adolescents from the general population did not differ from one another with regard to the prevalence of any of the clusters.

Interpersonal Identity. For interpersonal identity, we ran the same procedure as we did for ideological identity. We again retained a five-cluster solution (see Figure 9.2) that strongly resembles the solution for ideological identity, and hence the one found in a large Dutch community sample (Crocetti et al., 2008a). This cluster solution was replicated in all three samples, separately. Cohen's kappa coefficient, indicating the degree of correspondence between the cluster solution obtained in the entire sample (including all three samples) and cluster solutions obtained in separate samples was high, with values ranging from .72 to 1.00. The five clusters were labelled in the same way as the highly comparable clusters for ideological identity were, and will therefore not again be discussed in terms of their meanlevels. Similar to the results we obtained for ideological identity, Fisher's Exact Test (using 
Monte Carlo approximation to deal with issues related to our small sample sizes) revealed that there were overall differences between samples in their distribution across the clusters ( $p$ $<.001)$. Post-hoc pair-wise comparisons between all possible pairs of groups indicated that especially the juvenile delinquents differed in a remarkable way from clinically referred youth and adolescents from the general population (see Table 9.3). Delinquents were far more likely to be in the moratorium cluster, and less likely to be in the achievement cluster when compared to clinically referred youth and adolescents from the general population. Clinically referred youth and adolescents from the general population did not differ from one another with regard to the prevalence of any of the clusters.

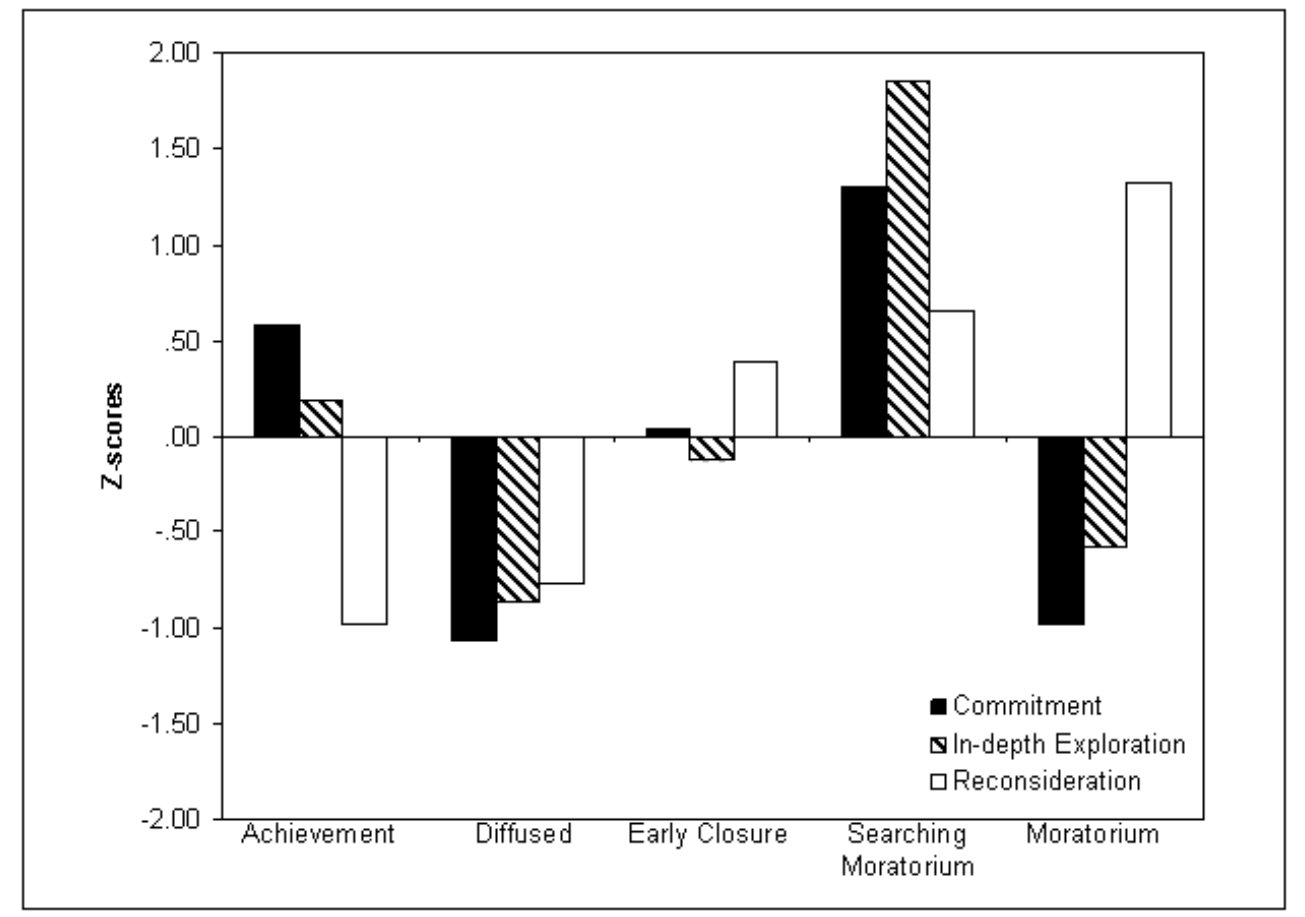

Figure 9.2

Final cluster-solution in the combined sample for interpersonal identity. Standardized means for commitment, in-depth exploration, and reconsideration. 
Table 9.3

Distribution of Adolescents from Clinical, Delinquent, and Comparison Samples across Five Interpersonal Identity Clusters

\begin{tabular}{lccc}
\hline & Clinical Sample & Delinquent Sample $(n=$ & Comparison Sample $(n=$ \\
& $(n=21)$ & $30)$ & $30)$ \\
\hline Early Closure & $4(19.0 \%)$ & $9(30.0 \%)$ & $9(30.0 \%)$ \\
Achievement & $11(52.4 \%)^{\mathrm{a}}$ & $2(6.7 \%)^{\mathrm{b}}$ & $11(36.7 \%)^{\mathrm{a}}$ \\
Searching Moratorium & $1(4.8 \%)$ & $2(6.7 \%)$ & $6(20.0 \%)$ \\
Diffused & $5(23.8 \%)$ & $3(10.0 \%)$ & $4(13.3 \%)$ \\
Moratorium & $0(0.0 \%)^{\mathrm{a}}$ & $14(46.7 \%)^{\mathrm{b}}$ & $0(0.0 \%)^{\mathrm{a}}$ \\
\hline
\end{tabular}

Note. Percentages represent the percentage of adolescents within a sample that is assigned to a cluster. Hence, percentages within a column add up to $100 \%$. When samples have different superscripts across rows, the proportion of individuals that were classified in a certain status was significantly different $(p<.05)$. Differences were assessed by the means of pair-wise comparisons (i.e., clinical sample - delinquent sample, clinical sample - comparison sample, and delinquent sample - comparison sample) with Fisher's Exact Tests, using Monte Carlo approximation.

\subsection{Discussion}

In the current study, we assessed whether juvenile delinquents experience problems with the key developmental task of adolescence: identity formation (Erikson, 1950). For that purpose, we compared identity formation in these youth to identity formation in clinically referred youth and adolescents from the general population.

Our findings indicate that male juvenile delinquents differed significantly from male clinically referred youth and male adolescents from the general population with regard to various identity processes for educational and friendship domains. First of all, juvenile delinquents tended to commit themselves to a lesser extent with regard to both education and friendships. Other findings were domain-specific. With regard to education, delinquents exhibited less reflection on their current commitments as they showed lowered levels of indepth exploration. For friendships, delinquents had more doubts about their current commitments as they exhibited much higher levels of reconsideration then clinically referred youth and adolescents from the general population did. Even though these findings already provide some insight into the problems juvenile delinquents face with regard to identity formation, an individuals' identity status is determined by examining one's amount of commitment relative to the degree to which an individual engages in exploration processes (Crocetti et al., 2008a, Marcia, 1966). Therefore, we proceeded to assess the prevalence of 
identity statuses based on all three identity processes among the three groups that were distinguished in this study.

For both educational and relational identity formation, clinically referred youth and adolescents from the general population were most often classified in the achievement status and the early closure status. Adolescents in the achievement status have reached satisfying commitments and they thoroughly reflect on these commitments. Achieved adolescents tend to display the lowest levels of problem behavior symptoms (e.g., Crocetti et al., 2008a). Adolescents in the early closure status, which is comparable to Marcia's (1966) foreclosure status, are to some extent committed, but they do not engage in thorough exploration and reflection on their commitments. Adolescents in such a state of identity usually display low levels of problem behavior (e.g., Crocetti et al., 2008a). Nevertheless, our results do indicate that a substantial proportion of youth from the general population and clinically referred youth yet need to engage in more thorough exploration activities in order to move towards the most desirable identity status, identity achievement (Marcia, 1966). Because much identity formation takes place in late adolescence and emerging adulthood (Arnett, 2004; Waterman, 1982), they still have plenty of time to move towards an achieved identity. Another substantial group of adolescents are already engaged in such a process, as they are classified in the searching moratorium status. Individuals in this status engage in extensive exploration from a relatively secure base provided by strong commitments. Available evidence points out that the searching moratorium status represents the positive facet of moratorium, as adolescents in this status feel certain and are satisfied with regard to their commitments, but nevertheless keep on looking for something better (Crocetti et al., 2008a).

In both identity domains that were distinguished in the current study (i.e., education and friendships), delinquent youth had a much less favorable identity status. Delinquents were more often classified as diffused with regard to ideological (i.e., educational) identity than youth from the general population, which suggests that delinquents tend to postpone educational identity related issues. For both ideological and interpersonal identity, juvenile delinquents were underrepresented in the achievement status, and often displayed the negative side of moratorium, as they were overrepresented in a maladaptive moratorium status. Individuals in this status lack a stable sense of commitment, do not reflect on their current commitments, but merely keep on looking for alternatives without committing to one of the available options. Erikson (1950) has argued that the stress associated with a lack of commitments can cause adolescents to engage in delinquent acts. This would lead to the conclusion that difficulties in identity formation caused youth to become delinquent and, 
hence, end up in detention centers. On the other hand, being in a juvenile detention center imposes several developmental constraints on adolescents which might further obstruct their identity formation (Greve, 2001). Thus, identity formation could both be cause and effect of detention. However, the clinically referred youth examined in the current study face a similar situation as juvenile delinquents, because they also reside in a residential institution. Living in such an institution might impact their opportunity to freely explore their identities and could frustrate their attempts to find satisfying commitments in a similar way in which identity formation among juvenile delinquents seems to be blocked. Yet, clinically referred youth did exhibit little exploration, but they did have stronger commitments. Thus, juvenile delinquents' lacking sense of commitment can not be solely attributed to their current living situation. Therefore, our findings suggest that the lacking sense of identity commitment among the juvenile delinquents examined in the current study might be a cause rather than an effect of their incarceration. In order to really determine causal relationships between delinquency and identity formation longitudinal data is needed. All the same, our data do suggest that identity formation is largely disturbed in juvenile delinquents. Given that previous empirical (e.g., Crocetti et al., 2008a; Meeus et al., 1999) and theoretical work (Erikson, 1950) has repeatedly stressed that a stronger sense of commitment is associated with less psychosocial problems, juvenile delinquents' lacking sense of identity commitment deserves the attention of both researchers and clinicians.

\section{Strengths and Limitations}

The current study is, to our knowledge, the first to compare identity formation of juvenile delinquents, clinically referred youth, and adolescents drawn from the general population. This three-group research design had many advantages. Because both the juvenile delinquents and clinically referred youth we included in this study were institutionalized, whereas only the juvenile delinquents differed from the non-institutionalized comparison sample, our results strongly suggest that juvenile delinquents' problems with identity formation are not entirely due to their institutionalization. Thus, our three-group design can be perceived as a means to adjust for the effects of institutionalization. Despite the strengths of the study, several limitations need to be recognized.

First, the current study employs only small samples. A reason for this is that it is hard to approach juvenile delinquents and clinically referred youth for research purposes, because youth care institutions and juvenile detention centers want to limit the burden on these adolescents. Two of the co-authors of the current study had connections with the participating 
institutions, which allowed us to reach these vulnerable youth. Although differences between detention centers should be minimal because the Dutch juvenile justice system is highly structured (Dutch Department of Justice, 2008), our findings could be somewhat biased because they were obtained in only one detention center. Replication of our findings in different detention centers is needed.

A second limitation is the cross-sectional nature of the current study. As previously mentioned, cause and effect of identity formation and delinquency could not be examined in this study. Therefore, we strongly encourage researchers to examine identity formation longitudinally in at-risk youth as well as among youth who already are in detention in order to individuate causal explanations.

A third limitation of the current study concerns its lack of more detailed background information on juvenile delinquents and clinically referred youth. Unfortunately, we were unable to examine what the exact reason for their institutionalization was due to privacy policies of institutions. Information about the exact clinical diagnosis that caused youth to be institutionalized or the exact delinquent act that had put them in detention would have allowed us to make a more detailed differentiation among institutionalized youth. The length of the sentence of juvenile delinquents might also have an impact, as it might be worthwhile for adolescents with longer sentence (e.g., a 3-year sentence) to commit themselves to life (including education and friends) in a detention center, whereas adolescents with a shorter sentence (e.g., 3 months) may feel less of an urge to commit themselves. Future studies should therefore try to get more detailed background information.

The fact that the current study only employed samples from the Netherlands can be perceived as a fourth limitation. The political and social system in the Netherlands is quite different from the political and social system of the United States. For example, soft drug use (e.g., marijuana, hash) is not prohibited in the Netherlands, which implies that the delinquents in the current study could not have been convicted for soft drug use related issues. On the other hand, regulations concerning firearms are much stricter in the Netherlands than they are in the United States. As a result, firearm availability is lower which could, in turn, affect the prevalence of the firearm-related type of crimes (Hepburn \& Hemenway, 2004). In addition, youth detention centers in the Netherlands offer juvenile delinquents educational and psychological care programs aimed at fostering a healthy development (Dutch Department of Justice, 2008). These programs might affect the identities of youth in a different way than the programs offered in youth detention centers in the United States do. Thus, cross-national replications of the results we obtained would be valuable. 
Identity Formation in Three Groups

Finally, we only focused on boys. Because previous studies (e.g., Klimstra, Hale, Raaijmakers, Branje, \& Meeus, 2009) have demonstrated substantial gender differences in adolescent identity formation, future studies should also include girls.

Notwithstanding these potential limitations, the current study provides an important first step in the examination of identity formation among troubled youth. Our data showed that delinquent youth, and not clinically referred youth, seem to display the most severe problems with regard to identity formation. 
Chapter 10

General Discussion 


\section{General Discussion}

In the current dissertation, we examined the dynamics of two important processes with regard to an individual's self-concept: personality development and identity formation. The aim of this chapter is to discuss the findings obtained in the eight studies that comprise the current dissertation. For this purpose, we will first describe our results for all specific research questions one-by-one (paragraph 10.1). Second, we will elaborate on the meaning and implications of these findings in the "Conclusions and General Discussion" section (paragraph 10.2). Finally, strength and limitations (paragraph 10.3), possible directions for future research (paragraph 10.4), concluding remarks (paragraph 10.5) will be provided.

\subsection{Summary of Main Findings}

\subsubsection{Findings for Personality Development}

\subsubsection{How do adolescent's personalities develop?}

Maturation of personality in adolescence was examined in Chapter 2. For this purpose, we followed Roberts et al.'s (2001) recommendations to distinguish among different aspects of change. First, we examined changes in mean-levels of personality traits, by assessing whether, for example, adolescents can on average become more agreeable with age. Second, we examined whether rank-order stability of personality traits (i.e., degree to which interindividual differences are set) increased with age, as Costa and McCrae (1994) once predicted. The third aspect of maturation we examined was change in personality profile stability. This profile stability is calculated for every single person in a sample. It indicates the extent to which an individual's constellation of Big Five traits (i.e., a person's personality profile) is stable across time (e.g., Furr, 2008). Because girls have been found to mature earlier than boys with regard to pubertal development and brain development (e.g., Beunen et al., 2000; Petersen et al., 1988), we also assessed gender differences in personality development.

Latent Growth Models (e.g., Duncan et al., 1999) revealed mean-level increases in Extraversion, Agreeableness, and Openness to Experience for both adolescent boys and girls. Levels of Conscientiousness did not change for boys and girls. With regard to Emotional Stability, boys exhibited increases, whereas girls exhibited no changes. Thus, the direction of change is quite similar boys and girls in four of the Big Five personality traits. However, there 
were differences in the timing of change. Especially for Agreeableness and Openness, girls reached high levels at an earlier age than boys do. Although boys did catch up with girls, girls appear to reach 'mature' mean-levels of Agreeableness about two years before boys do.

Pearson test-retest correlations indicated that inter-individual differences in personality traits become much more set as adolescents grow older, as one-year test-retest correlations were almost twice as high in late adolescence when compared to early adolescence. These increases applied to both boys and girls. Inter-individual differences were much more set in early adolescent girls than in early adolescent boys, and boys did not catch up with girls. As such, inter-individual differences in personality traits were more set in girls when compared to boys throughout the entire period of adolescence.

Finally, $q$-correlations were used to calculate personality profile stability between each pair of adjacent measurement occasions (i.e., T1-T2, T2-T3, T3-T4, and T4-T5) for each individual in our sample. Following suggestions by Biesanz et al. (2003), these four profile stability coefficients were then used as input in a Latent Growth Model. This model indicated that personality profile stability increased as adolescents grew older, with girls reaching high levels of profile stability at an earlier age than boys did.

Overall, our findings indicate that there is strong evidence for maturation of personality in adolescence. As Caspi et al. (2005) predicted, mean-levels of most Big Five traits (i.e., Agreeableness, Extraversion and Openness) tend to increase. We also found strong evidence for Costa and McCrae's (1994) claim that inter-individual differences become more set with age. In addition, we found substantive increases in personality profile stability, as Roberts et al. (2001) hypothesized. Similar to studies on pubertal development and brain maturation, we found substantive gender differences suggesting that girls mature approximately two years before boys.

\subsubsection{How does the developing personality relate to the development of problem behavior} symptoms?

In chapter 2 we demonstrated that an adolescent personality changes substantively. It is, however, unclear how the developing personality relates to problem behavior symptoms. Therefore, we tested two competing hypothetic models in chapter 3: (1) the scar (or complication) model suggesting that problem behavior symptoms cause changes in personality traits; and, conversely, (2) the vulnerability (or predisposition) model suggesting that, in fact, it is certain personality characteristics that may put an individual at risk for developing problem behavior symptoms (Krueger \& Tackett, 2003; Tackett, 2006; Widiger et 
al., 1999). These two models were tested simultaneously with cross-lagged panel models (e.g., Burkholder \& Harlow, 2003). We related Big Five personality traits to both internalizing (i.e., depression) and externalizing (i.e., aggression) problem behavior. As such, we ran 10 cross-lagged panel models.

In 8 of these 10 models we found relations between personality and problem behavior symptoms. Of these 8 models, only 1 model indicated that problem behavior symptoms were a better predictor for personality than the other way around. However, 7 models indicated that personality traits and problem behavior symptoms had equal predictive influences on one another. None of the models indicated that personality was a better predictor for problem behavior than the other way around.

Overall, our findings suggest bidirectional relations between personality traits, and internalizing and externalizing problem behavior symptoms. These bidirectional effects not only suggest that problem behavior symptoms affects personality traits (i.e., scar or complication model) or that certain personality characteristics affect problem behavior symptoms (i.e., vulnerability or predisposition model) (Krueger \& Tackett, 2003; Tackett, 2006; Widiger et al., 1999), they also suggest a corresponsive or transactional process (Roberts et al., 2003; Roberts \& Caspi, 2003) in which personality traits that have been scarred by problem behavior symptoms put an individual at further risk for developing additional problem behavior symptoms. These additional problem behavior symptoms could then further scar one's personality. Thus, the findings noted in chapter 3 provide an important insight in the relationship between personality traits and problem behavior symptoms, as they suggest transactional processes between personality and problem behavior.

\subsubsection{Are traditional personality types replicable as trajectories of personality development?}

In chapter 4, we searched for a developmental typology of adolescent personality. Personality types (i.e., Resilients, Undercontrollers, and Overcontrollers; Block \& Block, 1980) typically represent an individual's standing on a set of personality traits at a particular point in time, whereas Block (1971) argued that personality types should reflect the way individuals manifest themselves during longer periods in the life course. As such, he argued for the study of types of personality development (i.e., types based on mean-levels and changes in personality traits) instead of just types of personality (i.e., types solely based on mean-levels of personality traits). Unfortunately, very few studies have followed Block's recommendations. 
In an attempt to revive Block's (1971) search for types of personality development, we applied Latent Class Growth Analysis (LCGA; Nagin, 1999, 2005) to five-wave longitudinal data on 923 early to middle adolescents. We demonstrated that Resilients, Undercontrollers, and Overcontrollers can also be regarded as different developmental trajectories of personality. Additional analyses showed that our developmental trajectories were related to problem behavior symptoms in a similar way as cross-sectionally derived personality types are. Thus, Resilients exhibited little problem behavior, Overcontrollers showed the highest levels of depressive symptoms, and Undercontrollers had the highest levels of (minor) delinquency. Overall, we demonstrated that Resilients, Undercontrollers, and Overcontrollers displayed distinct trajectories of personality development, but remained clearly distinguishable from one another across time.

\subsubsection{How do different aspects of personality profile stability relate to adjustment?}

Chapter 5 provided an attempt to relate personality profile stability (i.e., the degree to which an individual's constellation of Big Five traits remains stable across time) to psychological adjustment. For this purpose, we could not just relate profile stability to psychological adjustment (i.e., depression, delinquency, and self-esteem), because Furr (2008) had recently hypothesized that that it may not be just the stability of a personality profile that influences psychological adjustment. Furr (2008) explained this as follows: within a population meanlevels of Big Five traits might be quite stable across two subsequent measurement occasions. In that particular situation, the norm personality profile (i.e., a profile based on mean-level scores on all Big Five traits) is quite stable across time. When an individual has a personality profile that matches the norm profile at both measurement occasions, he or she would reflect high profile stability. However, in that case it is not clear whether it is the degree to which an individual's personality profile is similar to the norm personality profile (a measure referred to as within-time normativeness) that influences psychological adjustment, or the stability of that individual's personality profile. As such, Furr (2008) recommended that researchers should correct for within-time normativeness when analyzing the influence personality profile stability has on psychological adjustment.

Following Furr's recommendations, we distinguished among three specific aspects of profile stability and examined their influences on psychological adjustment. These three aspects of profile stability are: (a) overall stability (i.e., stability of a rank-ordered set of raw (i.e., "ordinary") trait scores within a person); (b) within-time normativeness (i.e., the degree to which an individuals personality profile matches the norm personality profile; (c) 
distinctive stability (i.e., stability of a rank-ordered set of centralized trait scores (raw traits scores of an individual - mean trait score within a sample) within a person).

Using longitudinal path models, we replicated findings obtained in previous studies (e.g., Asendorpf \& van Aken, 1991; Ozer \& Gjerde, 1989; Roberts et al., 2001) by demonstrating that overall personality stability was strongly related to psychological adjustment (positively related to self-esteem, and negatively related to depression and delinquency). We then examined which component of overall personality stability was responsible for the relations with adjustment: within-time normativeness and/or distinctive stability. Our results revealed that there were no relations between distinctive stability and psychological adjustment, whereas within-time normativeness had strong positive associations with psychological adjustment (i.e., positive associations with self-esteem, and negative associations with depression and delinquency). These results confirmed Furr's (2008) hypothesis, by showing that it is not the stability of personality profiles as such, but the degree to which an individuals personality profile matches the profile of the "average" person within a sample that drives the associations with psychological adjustment.

\subsubsection{What are the correlates of off-time personality development?}

In chapter 2 we demonstrated that an adolescent's personality tends to mature as he/she grows older. However, there are substantive inter-individual differences in patterns of adolescent personality change. Chapter 6 focused on personality development that deviates from the average pattern, as we examined the correlates of hypermaturity (i.e., being ahead of one's peers) and immaturity (i.e., lagging behind one's peers) in personality development.

Hypermaturity and immaturity of personality had not been assessed before, as it is difficult to assess whether one is ahead or behind one's peers in development on a construct comprising multiple traits (i.e., the Big Five). However, Furr's (2008) concept of normativeness (see chapter 5) provides the means to relate the profile of an older individual (e.g., a 20-year old) to the profile of an "average" younger individual (e.g., the "average" 12year old) to calculate immaturity, and the other way around (relating the profile of a 12-year old to the profile of an "average" 20-year old) to calculate hypermaturity. To examine the correlates of this hypermaturity and immaturity, we calculated hypermaturity for early adolescents and immaturity for late adolescents. As previous studies found substantive gender differences in personality (e.g., chapter 2), we ran the analyses for boys and girls separately. Our analyses revealed that hypermaturity was related to internalizing problems in both boys and girls, whereas hypermature girls also exhibited higher levels of conflict with their parents. 
Immaturity was related to lower levels of anxiety in both boys and girls. Immature boys also exhibited lower levels of depression. However, immature girls exhibited higher levels of delinquency.

In sum, hypermaturity and immaturity of personality seem to be interesting new concepts with compelling relations to psychological adjustment. Hypermaturity seems to be predominantly related to internalizing problems, whereas immaturity is reflected in some sort of fearlessness (indicated by low levels of anxiety). In girls, this fearlessness is combined with externalizing problems. For boys, this fearlessness does not seem to have adverse effects.

\subsubsection{Identity Formation}

\subsubsection{What is the course of adolescent identity formation?}

Marcia (1966) proposed that adolescent identity formation was guided by two processes: exploration of and commitment to choices in relevant identity defining domains. Meeus refined Marcia's (1966) model by proposing three identity formation processes: commitment to choices, reconsideration of these choices, and in-depth exploration of one's current commitments (Crocetti, Rubini, \& Meeus, 2008). The purpose of the study described in chapter 7 was to examine developmental changes in these three processes in the period from early to late adolescence. Similar to our analysis strategy that we employed in the study described in chapter 2 , we analyzed these three identity formation processes in respect to mean-level change, rank-order stability (i.e., the extent to which inter-individual differences were set), and profile stability. For all three measures of change and stability, we also examined gender differences.

Our results indicated that mean-levels of commitment of boys and girls did not change across time. However, mean-levels of in-depth exploration increased for boys and girls, but only in middle to late adolescence. Levels of reconsideration did not change for girls, but decreased for boys. These decreases were the strongest in early to middle adolescence. Interindividual differences in identity dimensions did not become more set with age in adolescence. Girls exhibited much higher levels of profile stability in early adolescence when compared to boys. However, girls did not exhibit increases in profile stability towards late adolescence, whereas boys did. As a result, boys caught up with girls in late adolescence.

Taken as a whole, our results suggest differences between boys and girls in identity formation. Boys seemed to be lagging behind on girls in early to middle adolescence, but caught up with them in late adolescence. As such, there seemed to be similar developmental 
timing differences in identity formation for boys and girls as we found in our study of personality development (see chapter 2).

\subsubsection{How do the short-term dynamics of adolescent identity formation operate?}

Few studies have analyzed longitudinal changes in adolescent identity formation. The few that have done so have mainly examined changes using long time intervals (for example, Luyckx et al (2006) used a 6 month interval and we, ourselves, used a 12 month interval in our study described in chapter 7). However, identity formation has been described as a dynamic and self-organizing process (Kunnen et al., 2001) leading Lichtwarck-Aschoff et al. (2008) to recommend analysis of the short-term dynamics of identity formation. We followed these recommendations in our study described in chapter 8 . In this study we analyzed the day-today influences commitment and reconsideration exerted on one another, and examined how day-to-day fluctuations in the adolescent identity formation processes of commitment and reconsideration affected subsequent levels of these processes. In this study we followed recommendations by Goossens (2001) to distinguish among two different identity domains of the adolescent identity formation process: namely, an ideological (i.e., education) domain and an interpersonal (i.e., relation with a best friend) domain.

Because this study required adolescents to fill in identity questionnaires for five consecutive days (and repeating this process three times a year), we wanted to limit the burden on the adolescents so that they would continue to participate in the study. We limited the burden on the adolescents by administering a revised version of our identity questionnaire (i.e., the Utrecht - Management of Identity Commitments Scale (U-MICS; Crocetti, Rubini, \& Meeus, 2008) that had a single-item for commitment and a single-item for reconsideration. Specific items were made for both the ideological and interpersonal identity domains.

In order to use this revised, single-item version of U-MICS, it first needed to be validated. Our analyses revealed that the single-item version of U-MICS commitment and reconsideration scales were reliable and had acceptable validity. With this knowledge, we then analyzed cross-lagged panel models to track the day-to-day influences the adolescent identity formation processes of commitment and reconsideration exerted on one another. Our models indicated that reconsideration was a better predictor for commitment than the other way around. These results, which applied to both ideological and interpersonal identity domains, suggest that having doubts about commitments (reconsideration) causes a weakened sense of commitment, and that having less doubts about commitments leads to stronger commitments. In a next step, we examined whether fluctuations in commitment and 
reconsideration predicted overall levels in these processes. For both interpersonal and ideological identity domains, more day-to-day fluctuations in reconsideration predicted lower subsequent levels of commitment and higher subsequent levels of reconsideration. Fluctuations in commitment were not related to subsequent levels of commitment and reconsideration.

Taken together, our study suggest that it is important to examine the short-term dynamics of adolescent identity formation, since this study demonstrated that reconsideration and commitment are related to one another on a day-to-day basis, and, furthermore that dayto-day fluctuations in reconsideration lead to a weakened sense of identity, indicated by lower levels of commitment and higher levels of reconsideration.

\subsubsection{What are the differences and similarities between adolescent identity formation in} juvenile delinquents, clinically referred youth, and adolescents from the general population? Numerous studies have examined associations between adolescent identity formation and adolescent problem behavior symptoms (e.g., Crocetti, Rubini, Luyckx, \& Meeus, 2008; Crocetti, Rubini, \& Meeus, 2008; Luyckx et al., 2005, 2008), but these studies typically examined such associations in generally psychologically well-adjusted samples. It is unclear whether identity formation and problem behavior symptoms are related in a similar way when the severity of problem behavior symptoms passes a certain threshold. To shed more light on this issue, we compared identity formation in three adolescent samples: juvenile delinquents, clinically referred youth, and youth from the general adolescent population. Similar to our analysis strategy employed in our study described in chapter 8, we made distinction between ideological (i.e., education) and interpersonal (i.e., relationship with the best friend) identity domains.

The results of this study revealed that both interpersonal and ideological identity formation in juvenile delinquents differed substantively from identity formation in clinically referred youth and youth drawn from the general adolescent population. Juvenile delinquents were overrepresented in maladaptive moratorium statuses characterized by little commitment and in-depth exploration, but high levels of reconsideration. In addition, they were underrepresented in adaptive achievement statuses characterized by high levels of commitment and in-depth exploration and little reconsideration. These effects could not be entirely attributed to juvenile delinquents' institutionalization, because clinically referred youth, who were also institutionalized, did not differ from the non-institutionalized youth from the general population. Thus, juvenile delinquents seem to have serious problems with 


\section{General Discussion}

identity formation, compared to both clinically referred youth and adolescents from the general population.

\subsection{Conclusions and General Discussion}

The studies in the current dissertation answer a wide range of research questions concerning the dynamics of personality development and identity formation in adolescence. The results of the studies that were conducted to help answer these research questions have just been summarized in section 10.1. We will go into more detail on the implications of our findings in this section (section 10.2). However, to give the reader an opportunity to see all the results at one glance, we will summarize them in Table 10.1 .

Table 10.1. Summary of the Main Findings of this dissertation

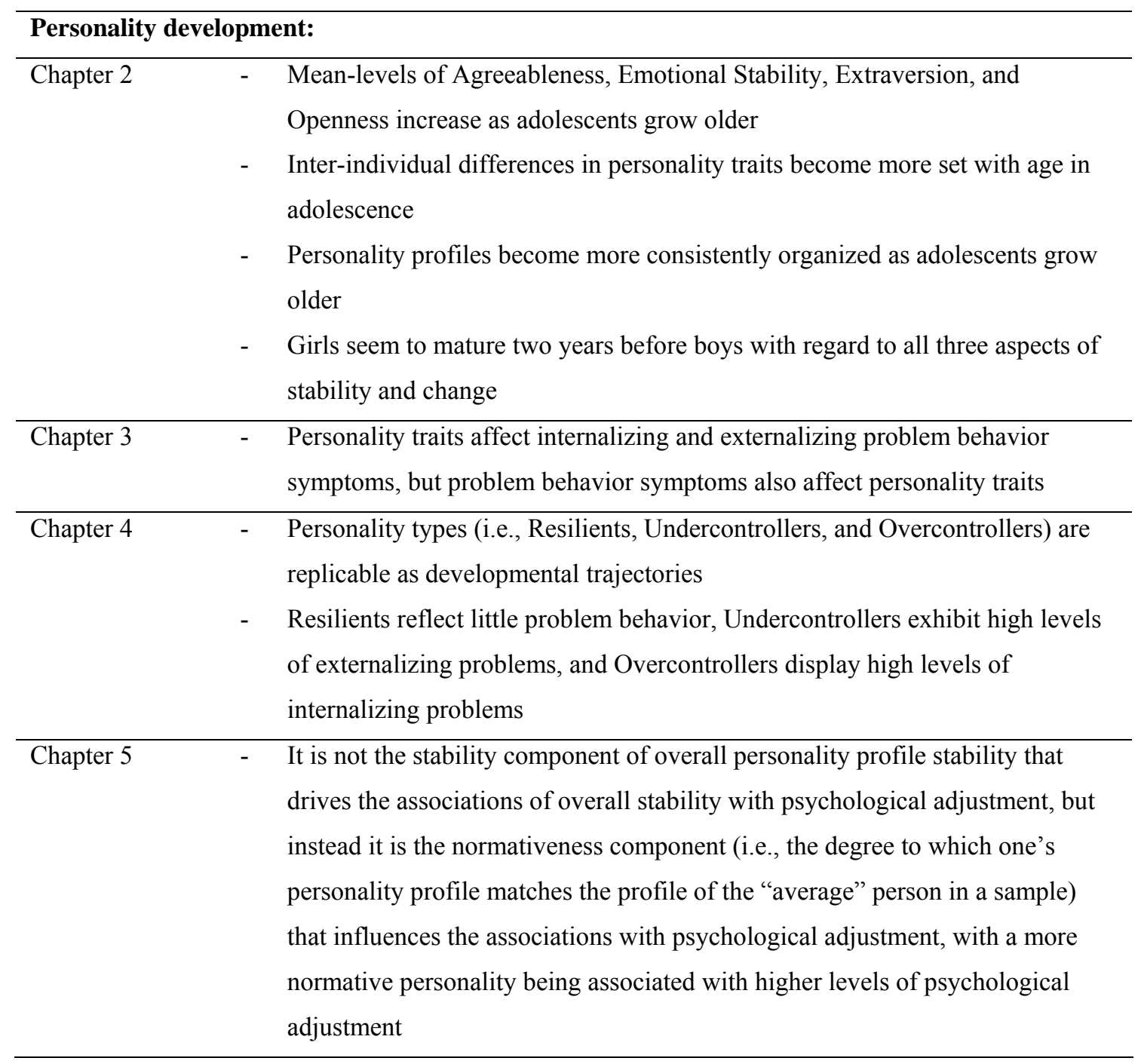




\begin{tabular}{|c|c|c|}
\hline Chapter 6 & - & $\begin{array}{l}\text { Hypermaturity is positively associated with internalizing problem behavior } \\
\text { symptoms in adolescent boys and girls, and with conflict with parents for girls, } \\
\text { but not for boys } \\
\text { Immaturity is negatively associated with internalizing problem behavior } \\
\text { symptoms for adolescent boys and girls, and positively associated with } \\
\text { externalizing problem behavior symptoms for girls, but not for boys }\end{array}$ \\
\hline \multicolumn{3}{|c|}{ Identity formation: } \\
\hline Chapter 7 & - & $\begin{array}{l}\text { Mean-levels of commitment are stable in early to late adolescence, but mean- } \\
\text { levels of reconsideration decrease in early to middle adolescence, and mean } \\
\text { levels of in-depth exploration increase in middle to late adolescence } \\
\text { Rank-order stability of identity dimensions does not change in early to late } \\
\text { adolescence } \\
\text { Identity profiles become more consistently organized as adolescents grow } \\
\text { older } \\
\text { Mean-level and profile development suggest that girls mature earlier than boys }\end{array}$ \\
\hline Chapter 8 & - & $\begin{array}{l}\text { The identity formation processes of commitment and reconsideration affect } \\
\text { one another on a day-to-day basis, with reconsideration being a better predictor } \\
\text { of commitment than the other way around } \\
\text { Day-to-day fluctuations in reconsideration affect subsequent levels of } \\
\text { commitment and reconsideration }\end{array}$ \\
\hline Chapter 9 & - & $\begin{array}{l}\text { Juvenile delinquents seem to have serious problems with identity formation, } \\
\text { compared to both clinically referred youth and adolescents from the general } \\
\text { population }\end{array}$ \\
\hline
\end{tabular}

\subsubsection{Personality Development}

The focus of the first part of the current dissertation (Chapters 2 to 6) was on the dynamics of personality development in adolescence and young adulthood. Following suggestions by Block (1971) and Roberts et al. (2001), we employed a wide range of variable-centered and person-centered statistical techniques to gain an encompassing perspective on personality development. Using variable-centered approaches, one can examine general trends in a population. With person-centered approaches, the way a set of traits is organized within a single person, the extent to which the organization of a set of traits changes across time, and individual differences in the organization of a set of traits can be examined. We will now first discuss our findings on personality development obtained with variable-centered approaches, and then move to a discussion of our findings obtained with person-centered approaches. 


\subsubsection{Variable-Centered Approaches to Personality Development}

Chapters 2 and 3 provide important insights in how the Big Five personality traits develop on average within a sample, and how this development relates to problem behavior symptoms. In chapter 2, mean-level increases in Extraversion, Agreeableness, and Openness to Experience for adolescent boys and girls, and increases in Emotional Stability for boys but not for girls, suggest a maturation process which could be described in terms of social investment (Roberts $\&$ Wood, 2006). Two of the traits in which we found changes have been shown to be related to social investment (i.e., Agreeableness and Emotional Stability; Lodi-Smith \& Roberts, 2007) whereas another one (i.e., Extraversion; Roberts et al., 2005) has been hypothesized to indicate social investment. Social investment refers to investment in and commitment to adult social developmental tasks, such as getting a job, establishing a romantic relationship, and starting a family. Thus, changes in Agreeableness, Emotional Stability, and Extraversion appear to be indicative of a maturation process in which adolescents become increasingly more ready to enter the adult social world. Openness is not associated with social investment, but has been shown to be strongly related to most advanced identity status: identity achievement (Crocetti, Rubini, \& Meeus, 2008; Luyckx et al., 2005). A well-developed sense of identity has, in turn, been theorized to be beneficial for resolving the subsequent developmental task: the development of intimacy (Erikson, 1950). Hence, Openness should at least have an indirect association with positive social adjustment.

Thus, an adolescent's mean levels of personality traits appear to change towards maturation as he/she grows older. In addition, a substantive increase of the rank-order coefficients from early to late adolescence indicates that inter-individual differences in personality traits became more set with age, as Costa \& McCrae (1994) already predicted. This suggests that the least agreeable, least conscientious, and least extraverted early adolescent could be among the most agreeable, most conscientious, and most extraverted individuals by middle adolescence. However, it is much less likely that such substantive relative personality changes take place after middle adolescence.

While we have already described how changes in adolescent personality traits could be related to (future) social adjustment, it is also important to note the associations between personality traits and problem behaviour symptoms. These associations were tested in our study described in chapter 3. Our findings suggested a transactional process in which problem behavior symptoms scar one's personality, and a scarred personality leaves adolescents vulnerable to the development of further problem behavior symptoms. Thus, both scar and vulnerability effects (Krueger \& Tackett, 2003; Tackett, 2006; Widiger et al., 1999) appear to 
be present. The corresponsive principle (Roberts et al., 2003; Roberts \& Caspi, 2003) could further explain these reciprocal relations between personality traits and problem behavior symptoms. According to this principle, personality development is guided by two mutually supportive processes: 'social selection' in which an individual selects an environment that matches his or her personality, and 'social influence' in which experiences related to one's chosen environment further shape one's personality. In the study described in chapter 3 , the corresponsive principle could be responsible for the reciprocal effect between Extraversion and Emotional Stability, and depressive symptoms. Individuals low on Extraversion and Emotional Stability are usually found to be prone to developing internalizing problem behavior symptoms (Akse et al., 2004), such as depression and anxiety (i.e., 'psychopathological selection'). These problems may cause them to avoid public places and other people, which may enhance their problem behavior symptoms, consequently deepening their introverted and neurotic personality characteristics (i.e., 'psychopathological influence').

In the studies described in chapter 2 and 3 personality is analyzed trait-by-trait. Even though this strategy provides an in-depth perspective on how specific parts of the adolescent personality develop and are related to problem behavior symptoms, Allport (1954) indicated that studies on personality studies should also attempt to examine how personality traits relate to one another within a single person. For that reason, we will now move to approaches that focus on particular patterns of personality traits within individuals. In other words, we will now discuss person-centered approaches to personality.

\subsubsection{Person-Centered Approaches to Personality Development}

In our study described in chapter 2, we examined how personality traits change on average within a sample. However, our results indicated that there were inter-individual differences in levels and changes of personality traits. These inter-individual differences can be captured by typological approaches. In the most the common one of these approaches, three personality types are distinguished: Resilients (who are generally well-adjusted), Undercontrollers (who have a tendency towards externalizing problems), and Overcontrollers (who have a tendency towards internalizing problem behavior) (Block \& Block 1980; Robins et al., 1996). Typological approaches tend to focus on how personality types manifest themselves on a particular point in time, whereas Block (1971) initially argued that personality types should represent how individuals represent themselves across longer periods of time. In our study described in chapter 4 we followed these recommendations and showed that Resilients, Undercontrollers, and Overcontrollers also appear as developmental trajectories of personality 


\section{General Discussion}

and that these developmental types were related to problem behavior symptoms in a similar way as has been found in cross-sectional studies. Thus, Resilients displayed little problem behavior symptoms, Undercontrollers had the highest levels of externalizing problem behavior symptoms (i.e., delinquency), and Overcontrollers had the highest levels of internalizing problem behavior symptoms (i.e., depression).

Typological approaches are very useful for capturing inter-individual differences in the patterning of personality traits. However, an important limitation of any typology is that boundaries between types are, at best, fuzzy (Asendorpf et al., 2001; Johnson et al., 2007). Therefore, it might be advisable to not use categorical person-centered approaches to personality, but use continuous person-centered measures. In the studies described in chapters 2, 4, 5, and 6 we have employed such measures.

In the study described in chapter 2 we briefly touched upon continuous personcentered measures of change and stability, as we examined changes in the stability of Big Five trait profiles within each participant across time. We demonstrated that individual's personality profiles become more stable, and hence more consistently organized as adolescents grow older. Increases in profile stability are thought to indicate maturation (Roberts et al., 2001), but this assertion is mainly theoretical. There are few studies relating personality profile stability to problem behavior, and most of these studies have only examined correlations and did not examine the direction of effects between these two constructs. For that reason, we set out to examine the longitudinal associations between personality profile stability and problem behavior symptoms in the study described in chapter 5.

After the publication of the study presented in chapter 2, a study by Furr (2008) was also published. He proposed that researchers should account for personality profile normativeness (i.e., the degree to which an individual's personality profile matches the normative profile (i.e., a profile base on mean trait scores in a sample)) when examining relations between personality profile stability and problem behavior symptoms, because it might very well be that associations between personality profile stability and problem behavior symptoms disappear when one would correct for this personality profile normativeness. We confirmed Furr's expectations, as our findings strongly suggested that it was not stability of personality that mattered for adjustment. Instead, it turned out that it was the degree to which one's personality profile matches the profile of the "average" person within a sample (i.e., personality profile normativeness) that was associated with adjustment. These associations between normativeness and adjustment could reflect a mechanism 
described by Roberts et al. (2006), in which compliance with social norms elicits social approval. Non-compliance (i.e., a non-normative personality profile) would be associated with withdrawal of social approval, which could lead to negative outcomes such as loosing one's job or one's friends. These losses could, in turn, lead to maladjustment.

All in all, the study described in chapter 5 provides an important new person-centered perspective on personality. Especially personality profile normativeness provides important new opportunities for person-centered personality research. For example, it is not only possible to calculate the similarity between a person's personality profile and a normative profile of same-aged peers, as one could also calculate the similarity of a person's personality profile and the normative profile of much younger or older individuals. In the study described in chapter 6 , we followed this approach by calculating hypermaturity and immaturity of personality. Because we had demonstrated in the study described in chapter 2 that there were substantive gender-differences in personality development, we calculated hypermaturity and immaturity for boys and girls separately. Indices of hypermaturity and immaturity of personality turned out to be strongly associated with problem behavior symptoms. Hypermaturity was associated with higher internalizing problems in boys and girls, and to higher levels of conflict with parents in girls, but not in boys. The stage-environment fit perspective (e.g., Eccles et al., 1993) could explain these findings. Hypermature adolescents are perhaps treated in a way they no longer perceive as age-inappropriate (i.e., childish). This could lead to despair (indicated by internalizing problem behavior symptoms in boys and girls) and frustrations (indicated by high levels of conflict with parents in girls).

Immaturity was associated with lower levels of internalizing problem behavior symptoms (i.e., anxiety) in both boys and girls. This could indicate some sort of fearlessness. In boys, this fearlessness had no adverse consequences, as they also reflected lower levels of depressive symptoms. For girls, the fearlessness associated with immaturity did have adverse consequences, as immature girls also reflected high levels of delinquency. The absence of adverse effects of immaturity in boys might be due to social acceptance of immaturity in men. Men tend to take up tasks of adult social life, such as marriage and starting a family, approximately three years later then women do (Statistics Netherlands, 2009a; 2009b). As such, it might be socially acceptable for men to behave in a somewhat immature way well into adulthood.

Overall, hypermaturity and immaturity of personality seem to be interesting new concepts in the study of personality development, given their strong relations with psychological adjustment in adolescent girls. Indices of off-time development are continuous 


\section{General Discussion}

person-centered measures. As such, they can be easily entered into a wide range of statistical analyses. This gives them an important advantage over categorical person-centered measures, such as the development personality types described in chapter 4. However, they still only provide a rough estimate of off-time personality development. Nevertheless, the study described in chapter 6 provides a first glimpse on what the consequences of off-time personality development could be.

\subsubsection{Identity Formation}

The second part of the current dissertation comprised three studies on adolescent identity formation (Chapter 7, 8, and 9). Identity formation takes place on different levels. It has been argued that one should distinguish among macro-levels (i.e., indicative of more general attitudes towards identity formation) and micro-levels (i.e., one's attitude towards identity formation during the last day) (Lichtwarck-Aschoff et al., 2008). Until now, few studies had examined developmental changes in identity formation at the macro-level with multiple measurement occasions (i.e., three or more measurement waves) covering the period from early to late adolescence, whereas even less studies had examined micro-level processes in identity formation. To shed more light on developmental changes in identity formation, the studies described in chapters 7 and 8 focused on the longitudinal dynamics of identity formation at the macro- and the micro-level, respectively. Identity formation has been shown to be related to problem behavior symptoms in the general adolescent population (Meeus et al., 1999). This would suggest that adolescents facing severe problem behavior symptoms (i.e., juvenile delinquents and clinically referred youth) also face problems with identity formation. To test whether this was the case, we compared identity formation in juvenile delinquents, clinically referred youth, and adolescents from the general population in the study described in chapter 9. We will now first discuss our studies on changes in identity formation, and then move to a discussion of identity formation in different groups of adolescents.

\subsubsection{The Course of Adolescent Identity Formation: Long- and Short-Term Change and}

\section{Stability}

In theoretical work (Erikson, 1950, 1968) identity formation has been described as a developmental process. It is therefore surprising that there is a limited amount of longitudinal studies on identity formation. In the study described in chapter 7 , we examined change and 
stability in the identity formation dimensions of commitment, in-depth exploration, and reconsideration from early to late adolescence. Our results suggested that early to middle adolescence is a period in which adolescent become more certain about their commitments, as their levels of reconsideration decreased. In other words, early to middle adolescence could be a period in which the commitment-formation cycle, described in a dual-cycle model of identity formation by Luyckx, Goossens, and Soenens (2006), is most active. In middle to late adolescence, we found increases in levels of in-depth exploration, which seems to reflect the second cycle in the dual-cycle model: the commitment-evaluation cycle in which adolescents actively think about their commitments. Levels of rank-order stability did not change from early to late adolescence. Thus, inter-individual differences in identity dimensions do not become more set in the period from early to late adolescence. A possible reason for this is that identity formation is initiated in adolescence, but a substantive amount of change takes place in young adulthood (e.g., Arnett, 2004; Luyckx, Goossens, \& Soenens, 2006). Thus, interindividual differences in identity formation may only become more set after adolescents have entered young adulthood. Finally, we also found evidence for small increases in profile stability, indicating that identity profiles become more consistently organized as adolescents grow older.

Overall, the findings of the study described in chapter 7 suggest that interesting changes in identity formation processes take place in adolescence. However, a limitation of this study is that it only focused on changes in dimensions of identity formation on a year-toyear basis. This is in agreement with other researchers such as Luyckx and colleagues (Luyckx, Goossens, \& Soenens, 2006; Luyckx, Schwartz, Goossens et al., 2008) who also focused on changes across longer periods of time (i.e., across 6-month intervals). However, because identity has been described as a dynamic and self-organizing system (Kunnen et al., 2001), Lichtwarck-Aschoff et al. (2008) recently recommended a focus on how identity formation processes operate on a micro-level (i.e., across shorter time-intervals). That is, they recommended a focus on day-to-day processes in identity formation. With the study described in chapter 8 we attempted to gain more insight in these short-term processes, by focusing on day-to-day influences between the two dimensions that seemed to be central to early adolescent identity formation (see chapter 7): commitment and reconsideration.

Our results indicated that commitment and reconsideration affected one another on a day-to-day basis, with reconsideration being a better predictor of commitment than the other way around. These results suggested that a search for new commitments may start with doubts (i.e., reconsideration) that weaken previous commitments, which, in turn, may lead to further 
doubts. Thus, we found evidence for a micro-level commitment-reconsideration dynamic, which had also been described by Meeus et al. (2010) from a macro-level perspective. Additionally, we demonstrated that day-to-day fluctuations in reconsideration predicted lower subsequent levels of commitment and higher subsequent levels of reconsideration. Fluctuations in commitment did not predict subsequent levels of commitment or reconsideration. Taken together, our findings on fluctuations in identity formation dimensions can be interpreted as support for Erikson's (1950) early conceptualization of identity formation as a search for sameness and continuity, as experiencing sameness and continuity with regard to identity (indicated by stable levels of reconsideration) was related to a stronger subsequent sense of identity (indicated by high levels of commitment and low levels of reconsideration). In sum, the study described in chapter 8 helps to demonstrate that identity formation indeed takes place on a micro (i.e., day-to-day) level, and that these microprocesses affect subsequent levels of identity commitment and reconsideration.

\subsubsection{Differences and Similarities in Identity Formation among juvenile delinquents,} clinically referred youth, and adolescents drawn from the general population The studies described in chapters 7 and 8 provide an interesting perspective on how identity processes develop on both the short term and the long term. However, both chapters 7 and 8 focused on generally well-adjusted individuals. Studies demonstrating linkages between identity formation and problem behavior symptoms in the general population (e.g., Crocetti, Rubini, \& Meeus, 2008; Crocetti, Rubini, Luyckx, \& Meeus, 2008; Luyckx et al., 2005) suggest that adolescents with severe behavioral problem symptoms could also display problems in identity formation. To further examine these issues, the study described in chapter 9 provides a comparison of identity formation in adolescent boys from the general adolescent population to identity formation processes in adolescent boys displaying severe problem behavior symptoms: juvenile delinquents and clinically referred youth.

From our results it appeared that both interpersonal and ideological identity formation were disturbed in juvenile delinquents, as they were largely overrepresented in maladaptive moratorium statuses (i.e., low levels of commitment and in-depth exploration, high levels of reconsideration), and underrepresented in adaptive achievement statuses (i.e., high levels of commitment and in-depth exploration, and low levels of reconsideration). These effects were not completely attributable to juvenile delinquents' institutionalization, because the institutionalized clinically referred youth displayed little problems with identity formation. Erikson (1950) asserted that problems with identity formation may lead to involvement in 
anti-social activities, such as delinquency. On the other hand, being in a harsh prison environment may also affect one's identity formation processes (Greve, 2001). Unfortunately, we could not examine cause and effect in this study because we only had cross-sectional data. All the same, our results show that juvenile delinquents face severe problems in both ideological and interpersonal identity formation.

\subsubsection{An Integrative Perspective on Identity Formation and Personality Development}

\section{in Adolescence}

The chapters in the current dissertation all focus on two key components of the self-concept in adolescence: personality and identity. Although all the studies described in the preceding chapters of this dissertation focus on either personality or identity, personality and identity are not examined in the same design. While this is an obvious limitation, the personality and identity studies of this dissertation do together provide a clear overall perspective on adolescent development of the self. To make this overall perspective more explicit, we will now discuss our findings on personality and identity in an integrative manner.

We found changes towards maturation, both in identity dimensions and in personality traits. In identity, these changes were apparent in decreasing doubts about commitments (i.e., reconsideration) in early to middle adolescence, and increasing reflection on current commitments (i.e., in-depth exploration) in middle to late adolescence. These changes were paralleled by increases in the personality traits Agreeableness, Emotional Stability, Extraversion, and Openness. The trait Openness to Experience contains assets such as intellect and curiosity, which have obvious linkages with a self-reflective and explorative process such as in-depth exploration. For that reason, strong associations have been found between in-depth exploration and Openness (Crocetti, Rubini, \& Meeus, 2008), which, in turn, explains why the increases we found in in-depth exploration (chapter 7) seem to be paralleled by increases in Openness (chapter 2).

Increases in Agreeableness, Emotional Stability, and Extraversion could, as we already indicated in Chapter 2, also be ascribed to adolescents getting ready to invest in and commit to social roles of adult life such as work and marriage (Roberts \& Wood, 2006; Lodi-Smith \& Roberts, 2007). Social investment theory basically focuses on what Freud once described as the two basic needs of human beings: "lieben und arbeiten" (love and work; see Erikson, 1950). In the study described in chapter 7 , we examined identity formation in two highly salient domains for adolescents: interpersonal relations (i.e., peer relations) and education. As 
commitment in peer relations has been shown to predict future commitment in romantic relations, adolescent interpersonal identity could be perceived as a precursor of adult interpersonal identity (i.e., romantic relations) (Meeus, Branje, van der Valk, \& de Wied, 2007; de Goede, 2009). When Bosma (1985) explored the saliency of commitments in a wide range of developmental domains relevant for adolescents, he found that many adolescents mentioned themes of education and future career orientation together. As such, commitment to education can definitely be considered as a developmental precursor of commitment to work. Taken together, investment and commitment in peer relations and education can be perceived as precursors of social investment in romantic relations (i.e., "lieben") and work (i.e., "arbeiten"). Because we already found increases in personality traits that have been associated with social investment in young adults (Agreeableness, Emotional Stability, and Extraversion; Roberts \& Wood, 2006; Lodi-Smith \& Roberts, 2007), our results suggest that social investment theory may also apply to adolescents.

Both boys and girls exhibit changes towards maturation in identity dimensions and personality traits, which suggests that they all get gradually more ready for entering adult social life. However, girls seem to be ahead on boys with regard to both identity and personality development. With regard to identity, girls had lower levels of reconsideration, higher levels of in-depth exploration, and more stable identity profiles than boys in early adolescence, but boys caught up with girls in many respects towards late adolescence. By late adolescence boys' identity profiles were equally stable as girls' profiles, and gender differences in levels of reconsideration also appeared to be smaller. For personality, gender differences in the timing of development were even more apparent. By middle adolescence, girls had already reached high levels of Agreeableness and Openness, and profile stability. Boys only reached such levels by late adolescence. The gender differences we found in the timing of personality development were in line with gender differences in pubertal timing (e.g., Beunen et al., 2000) and the timing of brain development (e.g., Lynn, 1994). Thus, girls appear to be ahead on boys in many aspects of adolescent development, including personality and identity.

Besides the striking similarities in changes and gender differences in these changes of identity and personality, both constructs also seem to be excellent markers for problem behavior symptoms. Previous studies had already shown that internalizing and externalizing problem behavior symptoms are related to identity formation (e.g., Crocetti, Rubini, \& Meeus, 2008), and in the study in chapter 9 we demonstrated that juvenile delinquents displayed much more problems with identity formation than clinically referred adolescents 
and adolescents from the general population. The studies in chapter 3, 4, 5, and 6 showed that problem behavior symptoms are also longitudinally related to personality traits, developmental personality types, the degree to which one's personality is similar to the personality profile of an "average" person (i.e., within-time normativeness), and to the timing of personality development. Our findings in chapter 3 even suggest that personality may play an important role in the etiology of problem behavior symptoms, as we found effects of personality on problem behavior symptoms and the other way around. Identity formation may have similar effects on problem behavior symptoms, and may also be affected by problem behavior symptoms. As such, identity formation may also play an important role in the etiology of problem behavior symptoms.

\subsection{Strengths and Limitations}

The studies comprising the current dissertation are characterized by several strengths. One important strength concerns the distinction between different aspects of change and stability (Caspi et al., 2005; Roberts et al., 2001) when examining development of personality (Chapter 2) and identity formation (Chapter 7). Distinguishing among three types of change and stability allowed us to show that development comprises much more than only changes in mean-levels of personality traits and identity dimensions. We also showed that interindividual differences become more set as rank-order stability increased, whereas increases in profile stability indicated that configurations of traits within individual adolescents become more consistently organized as they grow older. Considering mean-level change and rankorder stability alongside one another in the same study allowed us to conclude that the meanlevel personality changes that did occur in late adolescence should be perceived as normative changes that applied to a majority of individuals in our sample (Roberts \& DelVecchio, 2000). The usage of multiple measurement points when tracking development is another advantage of the studies presented in chapter 2 and 7. Five-annual wave two-cohort longitudinal data covering the entire period of adolescence allowed us to identify gender differences in the timing of personality development and identity formation that might otherwise have been overlooked. In addition, the more measurement occasions one uses, the more reliable the developmental trajectories one identifies become (Willett et al., 1998).

Other strengths included the employment of several state-of-the-art Structural Equation Modeling techniques that reduce biases due to measurement error. Among the techniques we employed are Latent Growth Models (Duncan et al., 1999; Chapters 2, 4, 6, 
and 7), Latent Class Growth Analysis (Nagin, 1999, 2005; Chapter 4), and cross-lagged panel models (Burkholder \& Harlow, 2003; Chapters 3, 5, and 8). These techniques should have lead to more reliable and conservative results in the studies of this dissertation, when compared to previous studies on personality and identity that employed less sophisticated techniques, such as Repeated Measures Analyis of Variance (ANOVA) or regression analyses.

In the studies described in chapters 5 and 6 we used very recent methodological refinements in the examination of personality profiles. Furr's (2008) recent distinction between overall personality profile stability and its subcomponents personality profile normativeness and distinctive personality profile stability allowed us to demonstrate that it is not the stability of a personality profile that is associated with psychological adjustment as such, but instead it is the degree to which one's personality profile matches the profile of the "average" person within a sample (personality profile normativeness) that is strongly related to psychological adjustment. In the study described in chapter 6 , we examined two interesting constructs related to personality profile normativeness that were briefly introduced by Furr (2008): hypermaturity (being ahead in personality development) and immaturity (lagging behind in personality development) of personality. These two concepts provided an interesting glimpse into the effects of off-time personality development.

Finally, in the study described in chapter 8 we used diary data on almost 600 adolescents that was collected on five consecutive days for three individual weeks during a year. Intensive data-collection on small groups of adolescents is becoming more common in the social sciences, but it is unusual to employ such an intensive design in a large-scale longitudinal study. These unique data allowed us to employ state-of-the-art longitudinal methodology to detect day-to-day processes in identity formation.

Besides these strengths, the studies in the current dissertation also have their limitations. As previously mentioned in paragraph 10.2.3., a first obvious limitation is that all the studies focus either on identity formation or on personality development despite important theoretical linkages between the two constructs. Unfortunately, longitudinal linkages between personality development and identity formation have until now only been examined in one study on emerging adulthood in women (Luyckx, Soenens, \& Goossens, 2006). Much more work is needed to uncover the longitudinal linkages between these two theoretically intertwined constructs.

Another limitation concerns our sole reliance on self-reported questionnaires. Identity formation concerns an internal and subjective process for which adolescents themselves are 
the best informants (Luyckx, Soenens, \& Goossens, 2006). However, in personality research, parental ratings of an adolescent's personality have also been employed (e.g., Selfhout, Denissen, Branje, \& Meeus, 2009). On the other hand, Soto et al. (2008) demonstrated that adolescent personality self-reports are reliable and valid from a psychometrical point-of-view. In addition, Robins et al. (2005) showed that individuals are well-able to judge how their personality has changed over a four-year period. Taken together, these studies suggest that self-reports on personality and identity are accurate methods for measuring these two intrapersonal constructs. Despite this, parental ratings of the adolescent's personality and identity formation may have provided us a fuller picture than our reliance on self-report alone.

Another limitation is the limited amount of attention we devoted to contextual factors, such as family and peer relations, and the adolescent's neighborhood, on the development of personality and identity. Family relations (Branje et al., 2004), peer relations (JensenCampbell et al., 2002), and the adolescent's neighborhood (Hart et al., 2008) have all been shown to be associated with personality. Identity formation has been shown to be associated with family relations (Beyers \& Goossens, 2008; Luyckx et al., 2007), but there is little research on other contextual factors that could have an impact on identity formation. This urged Bosma and Kunnen (2008) to recommend a focus on contextual factors when examining identity formation.

With our studies on identity formation (Chapter 7, 8 and 9), the strengths of one study can be perceived to be the limitations of the other studies. For example, the study described in chapter 7 provides a longitudinal perspective on changes in overall (i.e., macro-level) attitudes towards identity formation, but neglects the short-term (i.e., micro-level) processes in the study described in chapter 8 . The study described in chapter 8 , on the other hand, only employs one measurement occasion of macro-level identity formation. Combining short-term data with multiple measurement occasions of macro-level identity might be beneficial for our understanding of identity formation, because this would allow us how development on a macro-level is related to development on a micro-level.

Our use of two overlapping early to middle and middle to late adolescent cohorts to track longitudinal changes in personality (Chapter 2) and identity formation (Chapter 7), can also be perceived as a limitation. The two overlapping cohorts allowed for the use of accelerated Latent Growth Models (e.g., Duncan et al., 1999) in which one mean developmental trajectory for the entire period of adolescence can be estimated. In order to employ accelerated Latent Growth Models, the means of the cohorts at the point of overlap between these two cohorts (i.e., the last measurement occasion for the early to middle 


\section{General Discussion}

adolescent cohort and the first measurement occasion for the middle to late adolescent cohort) should be approximately equal. This appeared to be the case for identity dimensions (Chapter 7), but not for Big Five traits (Chapter 2). Thus, we were only able to employ accelerated Latent Growth Models in the study described in chapter 7. To obtain one mean developmental trajectory for adolescents it would be much better to follow the same individuals across the entire period of adolescence, and not rely on an accelerated design.

\subsection{Future Directions}

The just-mentioned strengths and limitations lead to several recommendations for future research. First of all, linkages between personality and identity formation need to be further examined, especially for the Social Investment Perspective (Lodi-Smith \& Roberts, 2007). The Social Investment Perspective explicitly states that personality traits and identity dimensions (especially commitment) in particular domains (i.e., romantic relations, occupation) should be related to one another. Although the Social Investment Perspective is mainly focused on young adulthood, we suggest in paragraph 10.2.3 that the perspective may also apply to adolescents. As such, future studies could examine the linkages between Big Five personality traits, and dimensions of adolescent identity domains such as friendships and education. Another direction of future research could be to examine parallels between identity and personality. It has been argued that Big Five personality traits should be perceived as stable core dimensions (Asendorpf \& van Aken, 2003), whereas identity dimensions have been described as surface traits that are more susceptible to change (Soenens et al., 2005). However, longitudinal studies have revealed that identity dimensions also tend to be remarkably stable across time (e.g., Chapter 7; Luyckx, Goossens, \& Soenens, 2006). This could suggest that identity dimensions are closer to the core of personality then has previously been assumed. To better explore this issue, future studies could examine the biological underpinnings (i.e., genetic factors, physiological factors such as the cortisol stress-hormone) of personality and identity to see whether identity and personality have similar biological origins. If biological factors account for a similar amount of variance in identity dimensions than in personality traits, identity dimensions should perhaps no longer be considered as surface personality traits.

The study described in chapter 8 provided a first glimpse on the nature of short-term processes in identity formation. We already found interesting short-term effects of commitment and reconsideration, but future studies could go much further with examining 
these short-term processes. For example, one could examine how psychological processes in significant others affect short-term identity formation processes, and how day-to-day changes in commitment and reconsideration are associated with psychological adjustment.

Finally, much more work is needed on the concepts of personality profile normativeness (Chapter 5) and personality profile immaturity and hypermaturity (Chapter 6). We already showed that these two concepts are interesting and strongly related to psychological adjustment, but future research needs to further explore these concepts. One could, for example, examine what drives an individual to obtain a normative personality profile, and what specific personal and contextual characteristics distinguish individuals with a normative personality profile from those with a non-normative profile. The concepts of immaturity and hypermaturity could perhaps be methodologically refined, because the indices we used in the study described in chapter 6 probably only provide rough estimates of off-time personality. With more refined hypermaturity and immaturity indices one could explore why girls seem to be more affected by immaturity when compared to boys, and whether hypermaturity and immaturity of personality have different effects at different ages.

\subsection{Concluding Remarks}

The aim of this dissertation was to disentangle the dynamics of personality development and identity formation in adolescence. First of all, changes in mean-levels of personality traits and identity dimensions were indicative of maturation. Inter-individual differences in personality traits became more set with age. Such changes were not evident in identity formation, but inter-individual differences in identity dimensions were rather stable across time. Personality and identity profiles also became more stable across time, suggesting that the indicators making up these constructs become more consistently organized within a person. We found gender differences in the timing of adolescent development, as early adolescent girls were ahead of early adolescent boys with regard to both personality development and identity formation. However, these gender differences declined as the adolescents grew older. With regard to identity, we also discovered active short-term influences between identity commitment and reconsideration, and that fluctuations in these processes affected subsequent levels of identity commitment and reconsideration of these commitments.

Both personality and identity appeared to be strongly related to problem behavior symptoms. With regard to personality, both internalizing and externalizing problem behavior symptoms were longitudinally associated with: (a) specific Big Five traits; (b) longitudinal 


\section{General Discussion}

trajectories of personality development; (c) the degree to which an individual's personality profile matches the profile of the "average" person within a sample (i.e., personality profile normativeness); (d) off-time personality development (i.e., hypermaturity and immaturity of personality). For identity formation, boys exhibiting severe externalizing problem behavior (i.e., juvenile delinquents) were more often classified in maladaptive identity statuses (i.e., moratorium), and less often in advantageous statuses (i.e., achievement) when compared to clinically referred boys and boys drawn from the general adolescent population.

Overall, adolescence is characterized by important changes towards maturation of personality and identity formation. The substantive associations between several aspects of personality and identity formation on the one hand, and problem behavior symptoms on the other hand, indicate the significance of personality and identity for psychological adjustment of adolescents. 


\section{References}

Achenbach, T. M., McConaughy, S. H., \& Howell, C. T. (1987). Child / adolescent behavioral and emotional problems: Implication of cross-informant correlations for situational specificity. Psychological Bulletin, 101,213-232.

Akse, J., Hale III, W. W., Engels, R. C. M. E., Raaijmakers, Q. A. W., \& Meeus, W. H. J. (2004). Personality, perceived parental rejection and problem behavior in adolescence. Social Psychiatry and Psychiatric Epidemiology, 39, 980-988.

Akse, J., Hale III, W. W., Engels, R. C. M. E., Raaijmakers, Q. A. W., \& Meeus, W. H. J. (2007a). Cooccurrence of depression and delinquency in personality types. European Journal of Personality, 21, 235-256.

Akse, J., Hale III, W. W., Engels, R. C. M. E., Raaijmakers, Q. A. W., \& Meeus, W. H. J. (2007b). Stability and change in personality type membership and anxiety in adolescence. Journal of Adolescence, 30, 813834.

Allport, G. W. (1954). Personality: A psychological interpretation (repr. ed.). New York: Henry Holt.

Arnett, J. J. (1999). Adolescent storm and stress, reconsidered. American Psychologist, 54 , 317-326.

Arnett, J. J. (2004). Emerging adulthood: The winding road from the late teens through the twenties. Oxford: University Press.

Asendorpf, J. B. (2003). Head-to-head comparison of the predictive validity of personality types and dimensions. European Journal of Personality, 17, 327-346.

Asendorpf, J. B., Borkenau, P., Ostendorf, F., \& van Aken, M. A. G. (2001). Carving personality description at its joints: Confirmation of three replicable personality prototypes for both children and adults. European Journal of Personality, 15, 169-198.

Asendorpf, J. B., \& van Aken, M. A. G. (1991). Correlates of the temporal consistency of personality patterns in childhood. Journal of Personality, 59, 689-703.

Asendorpf, J. B., \& van Aken, M. A. G. (1999). Resilient, overcontrolled, and undercontrolled personality prototypes in childhood: Replicability, predictive power, and the trait-type issue. Journal of Personality and Social Psychology, 77, 815-832.

Asendorpf, J. B., \& van Aken, M. A. G. (2003). Personality-relationship transaction in adolescence: Core versus surface personality characteristics. Journal of Personality, 71, 629-666.

Asendorpf, J. B., \& Denissen, J. J. A. (2006). Predictive validity of personality types versus personality dimensions from early childhood to adulthood: Implications for the distinction between core and surface traits. Merill-Palmer Quarterly, 52, 486-513.

Baerveldt, C. (2000). Pupil's networks in high schools: Network sampling, program and some results from a theory-oriented research project on petty crime of pupils. Paper presented at the $2^{\text {nd }}$ International Network Sampling Workshop, Maastricht, The Netherlands (March 2-4).

Baerveldt, C., van Rossem, R., \& Vermande, M. (2003). Pupils' delinquency and their social networks: A test of some network assumptions of the ability and inability models of delinquency. The Netherlands Journal of Social Sciences, 39, 107-125. 


\section{References}

Balistreri, E., Busch-Rossnagel, N. A., \& Geisinger, K. F. (1995). Development and preliminary validation of the ego identity process questionnaire. Journal of Adolescence, 18, 179-192.

Baltes, P. B., Reese, H. W., \& Lipsitt, L. P. (1980). Life-span developmental psychology. Annual Review of Psychology, 31, 65-110.

Baltes, P. B., Staudinger, U. M., \& Lindenberger, U. (1999). Lifespan psychology: Theory and application to intellectual functioning. Annual Review of Psychology, 50, 471-507.

Bennion, L. D., \& Adams, G. R. (1986). A revision of the extended version of the objective measure of ego identity status: An identity instrument for use with late adolescents. Journal of Adolescent Research, 1, 183-198.

Beunen, G., Thomis, M., Maes, H. H., Loos, R., Malina, R. M., Claessens, A. L., et al. (2000). Genetic variance of adolescent growth in stature. Annals of Human Biology, 27, 173-186.

Beyers, W., \& Goossens, L. (2008). Dynamics of perceived parenting and identity formation in late adolescence. Journal of Adolescence, 31, 165-184.

Biesanz, J. C., West, S. G., \& Kwok, O.-M. (2003). Personality over time: Methodological approaches to the study of short-term and long-term development and change. Journal of Personality, 71, 905-941.

Birmaher, B., Khetarpal, S., Brent, D., Cully, M., Balach, L., Kaufman, J., McKenzie Neer, S. (1997). The screen for child anxiety related emotional disorders (scared): Scale construction and psychometric characteristics. Journal of the American Academy of Child and Adolescent Psychiatry, 36, 545-553.

Björkqvist, K., Lagerspetz, K. M. J., \& Kaukiainen, A. (1992). Do girls manipulate and boys fight?:

Developmental trends in regard to direct and indirect aggression. Aggressive Behavior, 18, 117-127.

Block, J. (1971). Lives through time. Berkeley, CA: Bancroft books.

Block, J. H., \& Block, J. (1980). The role of ego-control and ego-resiliency in the organization of behavior. In W. A. Collins (Ed.), Development of cognition, affect, and social relations (Vol. 13, pp. 39-101). Hillsdale: Lawrence Erlbaum Associates.

Block, J., \& Robins, R. W. (1993). A longitudinal study of consistency and change in self-esteem from early adolescence to early adulthood. Child Development, 64, 909-923.

Bollen, K. (1989). Structural equations with latent variables. New York: Wiley.

Bosma, H. A. (1985). Identity Development in Adolescence: Coping with Commitments. Unpublished doctoral dissertation, University of Groningen.

Bosma, H. A., \& Kunnen, E. S. (2008). Identity-in-context is not yet identity development-in-context. Journal of Adolescence, 31, 281-289.

Branje, S. J. T., Frijns, T., Finkenauer, C., Engels, R., \& Meeus, W. (2007). You are my best friend: Commitment and stability in adolescents' same-sex friendships. Personal Relationships, 14, 587-603.

Branje, S. J. T., Van Lieshout, C. F. M., \& Gerris, J. R. M. (2007). Big five personality development in adolescence and adulthood. European Journal of Personality, 21, 45-62.

Branje, S. J. T., van Lieshout, C. F. M., \& van Aken, M. A. G. (2004). Relations between big five personality characteristics and perceived support in adolescents' families. Journal of Personality and Social Psychology, 86, 615-628.

Brooks-Gunn, J., Petersen, A.C., \& Eichorn, D. (1985). The study of maturational timing effects in adolescence. Journal of Youth and Adolescence, 14,149-161. 
Burkholder, G. J., \& Harlow, L. L. (2003). An illustration of a longitudinal cross-lagged design for larger structural equation models. Structural Equation Modeling, 10, 465-486.

Burwell, R. A., \& Shirk, S. R. (2007). Subtypes of rumination in adolescence: Associations between brooding, reflection, depressive symptoms, and coping. Journal Of Clinical Child And Adolescent Psychology, 36, 56-65.

Caldwell, D. F., \& Burger, J. M. (1998). Personality characteristics of job applicants and success in screening interviews. Personnel Psychology, 51, 119-136. 486.

Campbell, J. D. (1990). Self-esteem and clarity of the self-concept. Journal of Personality and Social Psychology, 59, 538-549.

Caprara, G. V., Barbaranelli, C., \& Zimbardo, P. G. (1996). Understanding the complexity of human aggression: Affective, cognitive, and social dimensions of individual differences in propensity toward aggression. European Journal of Personality, 10, 133-155.

Caspi, A., \& Roberts, B. W. (1999). Personality Continuity and Change Across the Life Course. In L. A. Pervin \& O. P. John (Eds.), Handbook of Personality: Theory and Research (2nd ed.). New York: Guilford Press.

Caspi, A., Roberts, B. W., \& Shiner, R. L. (2005). Personality development: Stability and change. Annual Review of Psychology, 56, 453-484.

Chen, F. F. (2007). Sensitivity of goodness of fit indexes to lack of measurement invariance. Structural Equation Modeling, 14, 464-504.

Cheung, G. W., \& Rensvold, R. B. (2002). Evaluating goodness-of-fit indexes for testing measurement invariance. Structural Equation Modeling, 9, 233-255.

Clark, D. B., Smith, M. G., Neighbors, B. D., Skerlec, L. M., \& Randall, J. (1994). Anxiety disorders in adolescence: Characteristics, prevalence, and comorbidities. Clinical Psychology Review, 14, 113-137.

Clark, L. A., Watson, D., \& Mineka, S. (1994). Temperament, personality, and the mood and anxiety disorders. Journal of Abnormal Psychology, 103, 103-116.

Clausen, J. S. (1991). Adolescent competence and the shaping of the life course. American Journal of Sociology, $96,805-842$.

Cohen, J. (1988). Statistical power analysis for the behavioral sciences (2nd ed.). Hillsdale, New Jersey: Erlbaum.

Cole, D. A., Martin, N. C., \& Steiger, J. H. (2005). Empirical and conceptual problems with longitudinal traitstate models: Introducing a trait-state-occasion model. Psychological Methods, 10, 3-20.

Cole, D. A., Nolen-Hoeksema, S., Girgus, J., \& Paul, G. (2006). Stress exposure and stress generation in child and adolescent depression: A laten trait-state-error approach to longitudinal analyses. Journal of Abnormal Psychology, 115, 40-51.

Colom, R., \& Lynn, R. (2004). Testing the developmental theory of sex differences in intelligence on 12-18 year olds. Personality and Individual Differences, 36, 75-82.

Compas, B. E., Malcarne, V. L., \& Fondacaro, K. M. (1988). Coping with stressful events in older children and young adolescents. Journal of Consulting and Clinical Psychology, 56, 405-411.

Connolly, J., Furman, W., \& Konarski, R. (2000). The role of peers in the emergence of 


\section{References}

heterosexual romantic relationships in adolescence. Child Development, 71, 1395-1408.

Costa, P. T., Jr., \& McCrae, R. R. (1992). Revised NEO Personality Inventory (NEO-PI-R) and the Five Factor Inventory (NEO-FFI): Professional manual. Odessa, FL: Psychological Assessment Resources.

Costa, P. T., Jr., \& McCrae, R. R. (1994). Set like plaster? Evidence for the stability of adult personality. In T. F. Heatherton \& J. L. Weinberger (Eds.), Can personality change? (pp. 21-40). Washington: American Psychological Association.

Côté, J. E., \& Levine, C. (1987). A formulation of erikson's theory of ego identity formation. Developmental Review, 7, 273-325.

Côté, J. E. \& Levine, C. (1988). A critical examination of the ego identity status paradigm. Developmental Review, 8, 147-184.

Craighead, W. E., Smucker, M. R., Craighead, L. W., \& Ilardi, S. S. (1998). Factor analysis of the children's depression inventory in a community sample. Psychological Assessment, 10, 156-165.

Crocetti, E., Rubini, M., Luyckx, K., \& Meeus, W. (2008). Identity formation in early and middle adolescents from various ethnic groups: From three dimensions to five statuses. Journal of Youth and Adolescence, 37, 983-996.

Crocetti, E., Rubini, M., \& Meeus, W. H. J. (2008). Capturing the dynamics of identity formation in various ethnic groups: Development and validation of a three-dimensional model. Journal of Adolescence, 31, 207-222.

Cui, M., Donnelan, M. B., \& Conger, R. D. (2007). Reciprocal influences between parents' marital problems and adolescent internalizing and externalizing behavior. Developmental Psychology, 43, 1544-1552.

De Fruyt, F., Bartels, M., Van Leeuwen, K. G., De Clercq, B., Decuyper, M., \& Mervielde, I. (2006). Five types of personality continuity in childhood and adolescence. Journal of Personality and Social Psychology, 91, 538-552.

De Fruyt, F., Mervielde, I., Hoekstra, H. A., \& Rolland, J. P. (2000). Assessing adolescents' personality with the neo pi-r. Assessment, 7, 329-345.

Degirmencioglu, S. M., Urberg, K. A., Tolson, J. M., \& Richard, P. (1998). Adolescent friendship networks: Continuity and change over the school year. Merrill-Palmer Quarterly, 44, 313337.

De Goede, I. H. A. (2009). Development of Adolescent Relationships. Doctoral Dissertation: Utrecht University, The Netherlands.

De Goede, I. H. A., Branje, S. J. T., Delsing, M. J. M. H., \& Meeus, W. H. J. (2009). Linkages over time between adolescents' relationships with their parents and friends. Journal of Youth and Adolescence (2009), doi: 10.1007/s10964-009-9403-2.

Denissen, J. J. A., Asendorpf, J. B., \& van Aken, M. A. G. (2008). Childhood personality predicts long-term trajectories of shyness and aggressiveness in the context of demographic transitions in emerging adulthood. Journal of Personality, 76, 67-99. 
Di Fabio, A., \& Busoni, L. (2007). Fluid intelligence, personality traits and scholastic success: Empirical evidence in a sample of italian high school students. Personality And Individual Differences, 43, 2095 2104.

Digman, J. M., \& Inouye, J. (1986). Further specification of the five robust factors of personality. Journal of Personality and Social Psychology, 50, 116-123.

Donnelan, M. B., Conger, R. D., \& Burzette, R. G. (2007). Personality development from late adolescence to young adulthood: Differential stability, normative maturity, and evidence for the maturity-stability hypothesis. Journal of Personality, 75, 237-264.

Dubas, J. S., Gerris, J. R. M., Janssens, J. M. A. M., \& Vermulst, A. A. (2002). Personality types of adolescents: Concurrent correlates, antecedents, and type x parenting interactions. Journal of Adolescence, 25, 7992.

Duncan, T. E., Duncan, S. C., Stryker, L. A., Li, F., \& Alpert, A. (1999). An introduction to latent variable growth curve modelling. Mahwah, NJ: Lawrence Erlbaum Associates, Inc.

Dutch Department of Justice [Ministerie van Justitie] (2008). Youth in the Juvenile Justice System [De Jeugdige in het Jeugdstraf(proces)recht]. Available from http://www.justitie.nl/onderwerpen/jeugd/jeugdcriminaliteit/

Eccles, J. S., Midgley, C., Wigfield, A., Buchanan, C. M., Reuman, D., Flanagan, C., \& Mac Iver, D. (1993). Development during adolescence: The impact of stage-environment fit on young adolescents' experience in schools and in families. American Psychologist, 48, 90-101.

Ehrler, D. J., Evans, J. G., \& McGhee, R. L. (1999). Extending Big-Five theory into childhood: A preliminary investigation into the relationship between the Big-Five personality traits and behavior problems in children. Psychology in the Schools, 36, 451-458.

Eichelsheim, V., Buist, K., Deković, M., Wissink, I., Frijns, T., van Lier, P., et al. (2009). Associations among the parent-adolescent relationship, aggression and delinquency in different ethnic groups: A replication across two Dutch samples. Social Psychiatry and Psychiatric Epidemiology (2009, doi 10.1007/s00127009-0071-z).

Erikson, E. H. (1950). Childhood and society. New York: Norton.

Erikson, E. H. (1968). Identity: Youth and crisis). New York: Norton.

Erikson, E. H. (1972). Childhood and society (Repr. ed.). Harmondsworth, Middlesex: Penguin Books.

Erikson, E. H. (1974). Identity: Youth and crisis (Repr. ed.). London: Faber and Faber.

Ferguson, E. (2000). Hypochondriacal concerns and the five factor model of personality. Journal of Personality, $68,705-724$.

Funder, D. C. (1995). On the accuracy of personality judgment: A realistic approach. Psychological Review, 102, 652-670.

Furman, W., \& Buhrmester, D. (1985). Children's perceptions of the personal relationships in their social networks. Developmental Psychology, 21, 1016-1024.

Furman, W., \& Buhrmester, D. (1992). Age and sex-differences in perceptions of networks of personal relationships. Child Development, 63, 103-115.

Furr, R. M. (2008). A framework for profile similarity: Integrating similarity, normativeness, and distinctiveness. Journal of Personality, 76, 1267-1316. 


\section{References}

Ge, X., \& Conger, R. D. (1999). Adjustment problems and emerging personality characteristics from early to late adolescence. American Journal of Community Psychology, 27, 429-459.

Geary, D. C., Byrd-Craven, J., Hoard, M. K., Vigil, J., \& Numtee, C. (2003). Evolution and development of boys' social behavior. Developmental Review, 23, 444-470.

Gerris, J. R. M., Houtmans, M. J. M., Kwaaitaal-Roosen, E. M. G., Schipper, J. C., Vermulst, A. A., \& Janssens, J. M. A. M. (1998). Parents, adolescents and young adults in dutch families: A longitudinal study. Nijmegen: Institute of Familiy Studies University of Nijmegen.

Geuzaine, C., Debry, M., \& Liesens, V. (2000). Separation from parents in late adolescence: The same for boys and girls? Journal of Youth and Adolescence, 29, 79-91.

Gibbs, J. C., Potter, G. B., Barriga, A. Q., \& Liau, A. K. (1996). Developing the helping skills and prosocial motivation of aggressive adolescents in peer group programs. Aggression and Violent Behavior, 1, 283-305.

Giedd, J. N., Blumenthal, J., Jeffries, N. O., Castellanos, F. X., Liu, H., Zijdenbos, A., et al. (1999). Brain development during childhood and adolescence: A longitudinal mri study. Nature Neuroscience, 2, 861863.

Gjerde, P. F., Block, J., \& Block, J. H. (1988). Depressive symptoms and personality during late adolescence: Gender differences in the externalization - internalization of symptom expression. Journal of Abnormal Psychology, 97, 475-486.

Goldberg, L. R. (1992). The development of markers for the big-five factor structure. Psychological Assessment, 4, 26-42.

Goossens, L. (2001). Global versus domain-specific statuses in identity research: A comparison of two selfreport measures. Journal of Adolescence, 24, 681-699.

Gore, P. A. Jr. (2000). Cluster analysis. In H. E. A. Tinsley \& S. D. Brown (Eds.), Handbook of applied multivariate statistics and mathematical modeling (pp. 297-321). San Diego, CA: Academic Press.

Graber, J. A. (2003). Puberty in context. In: Gender Differences at Puberty. Hayward, C. (ed.). New York: Cambridge University Press.

Graber, J. A., Lewinsohn, P. M., Seeley, J. R., \& Brooks-Gunn, J. (1997). Is psychopathology associated with the timing of pubertal development? Journal of the American Academy of Child and Adolescent Psychiatry, 36, 1768-1776.

Graziano, W. G., \& Ward, D. (1992). Probing the big five in adolescence: Personality and adjustment during a developmental transition. Journal of personality, 60, 425-439.

Greve, W. (2001). Imprisonment of juveniles and adolescents: Deficits and demands for developmental research. Applied Developmental Science, 5, 21-36.

Grotevant, H. D. (1987). Toward a process model of identity formation. Journal of Adolescent Research, 2, 203-222.

Grotevant. H. D., \& Adams, G. R. (1984). Development of an objective measure to assess ego identity in adolescence: Validation and replication. Journal of Youth and Adolescence, 13, 419-438.

Grotevant, H. D., \& Cooper, C. R. (1985). Patterns of interaction in family relationships and the development of identity exploration in adolescence. Child Development, 56, 415-428. 


\section{References}

Grotevant, H. D., Thorbecke, W., \& Meyer, M. L. (1982). An extension of Marcia’s identity status interview into the interpersonal domain. Journal of Youth and Adolescence, 11, 33-47.

Hale III, W. W., Raaijmakers, Q. A. W., Muris, P., \& Meeus, W. H. J. (2005). Psychometric properties of the screen for child anxiety related emotional disorders in the general adolescent population. Journal of the American Academy of Child and Adolescent Psychiatry, 44, 283-290.

Hale, W. W., III, Raaijmakers, Q., Muris, P., Van Hoof, A., \& Meeus, W. (2008). Developmental trajectories of adolescent anxiety disorder symptoms: A 5-year prospective community study. Journal of the American Academy of Child and Adolescent Psychiatry, 47, 556-564.

Hale III, W. W., VanderValk, I., Akse, J., \& Meeus, W. (2008). The interplay of early adolescents' depressive symptoms, aggression and perceived parental rejection: A four-year community study. Journal of Youth and Adolescence, 37, 928-940.

Hart, D., Atkins, R., \& Fegley, S. (2003). Personality and development in childhood: A person-centered approach. Monographs of the Society for Research on Child Development, 68, 1-109.

Hart, D., Atkins, R., \& Matsuba, M. K. (2008). The association of neighborhood poverty with personality change in childhood. Journal of Personality and Social Psychology, 94, 1048-1061.

Harter, S. (1990). Developmental differences in the nature of self-representations: Implications for the understanding, assessment, and treatment of maladaptive behavior. Cognitive Therapy and Research, 12, 113-142.

Heise, D. R. (1969). Separating reliability and stability in test-retest correlation. American Sociological Review, 34, 93-101.

Helson, R., Kwan, V. S. Y., John, O. P., \& Jones, C. (2002). The growing evidence for personality change in adulthood: Findings from research with personality inventories. Journal of Research in Personality, 36, 287.

Hepburn, L. M., \& Hemenway, D. (2004). Firearm availability and homicide: A review of the literature. Aggression and Violent Behavior, 9, 417-440.

Hix-Small, H., Duncan, T. E., Duncan, S. C., \& Okut, H. (2004). A multivariate associative finite growth mixture modeling approach examining adolescent alcohol and marijuana use. Journal of Psychopathology and Behavioral Assessment, 26, 255-270.

Hodges, K. (1990). Depression and anxiety in children: A comparison of self-report questionnaires to clinical interview. Psychological Assessment, 2, 367-381.

Hoekstra, H. A., Ormel, J., \& De Fruyt, F. (1996). NEO persoonlijkheids vragenlijsten: NEOPI-R, NEO-FFI. Handleiding [NEO personality questionnaires: NEO-PI-R and NEO-FFI-Manual]. Lisse, The Netherlands: Swets \& Zeitlinger.

Hu, L., \& Bentler, P. M. (1999). Cutoff criteria for fit indexes in covariance structure analysis: Conventional criteria versus new alternatives. Structural Equation Modeling, 6, 1-55.

Huey Jr., S. J., \& Weisz, J. R. (1997). Ego control, ego resiliency, and the five-factor model as predictors of behavioral and emotional problems in clinic-referred children and adolescents. Journal of Abnormal Psychology, 106, 404-415.

Hyde, J. S., Mezulis, A. H., \& Abramson, L. Y. (2008). The ABCs of depression: Integrating 


\section{References}

affective, biological, and cognitive models to explain the emergence of the gender difference in depression. Psychological Review, 115, 291-313.

Jensen-Campbell, L. A., Adams, R., Perry, D. G., Workman, K. A., Furdella, J. Q., \& Egan, S. K. (2002). Agreeableness, extraversion, and peer relations in early adolescence: Winning friends and deflecting aggression. Journal of Research in Personality, 36, 224-251.

Jensen-Campbell, L. A., \& Malcolm, K. T. (2007). The importance of conscientiousness in adolescent interpersonal relationships. Personality and Social Psychology Bulletin, 33, 368-383.

John, O. P., Caspi, A., Robins, R. W., Moffitt, T. E., \& Stouthamer-Loeber, M. (1994). The "little five": Exploring the nomological network of the five-factor model of personality in adolescent boys. Child Development, 65, 160-178.

Johnson, W., Hicks, B. M., McGue, M., \& Iacono, W. G. (2007). Most of the girls are alright, but some aren't: Personality trajectory groups from ages 14 to 24 and some associations with outcomes. Journal of Personality and Social Psychology, 93, 266-284.

Kasen, S., Chen, H., Sneed, J., Crawford, T., \& Cohen, P. (2006). Social role and birth cohort influences on gender-linked personality traits in women: A 20-year longitudinal analysis. Journal of Personality and Social Psychology, 91, 944-958.

Kasen, S., Cohen, P., Skodol, A. E., Johnson, J. G., Smailes, E., \& Brook, J. S. (2001). Childhood depression and adult personality disorder: Alternative pathways to continuity. Archives of General Psychiatry, 58, 231-236.

Keijsers, L., Frijns, T., Branje, S. J. T., Finkenauer, C., \& Meeus, W. (2010). Gender differences in keeping secrets from parents in adolescence. Developmental Psychology, 46, 293-298.

Keijsers, L., Frijns, T., Branje, S. J. T., \& Meeus, W. (2009). Developmental links of adolescent disclosure parental solicitation and control with delinquency: Moderation by parental support. Developmental Psychology, 45, 1314-1327.

Kernis, M. H., Grannemann, B. D., \& Barclay, L. C. (1989). Stability and level of self-esteem as predictors of anger arousal and hostility. Journal of Personality and Social Psychology, 56, 10131022.

Kernis, M. H., Grannemann, B. D., \& Barclay, L. C. (1992). Stability of self-esteem: assessment, correlates, and excuse making. Journal of Personality, 60, 621-644.

Kerpelman, J. L., \& Lamke, L. K. (1997). Anticipation of future identities: A control theory approach to identity development within the context of serious dating relationships. Personal Relationships, 4, 47-62.

Kerpelman, J. L., Pittman, J. F., \& Lamke, L. K. (1997). Toward a microprocess perspective on adolescent identity development: an identity control theory approach. Journal of Adolescent Research, 12, 325-346.

Kerpelman, J. L., \& Pittman, J. F. (2001). The instability of possible selves: Identity processes within late adolescents' close peer relationships. Journal of Adolescence, 24, 491-512.

Kerr, M., Tremblay, R. E., Pagani, L., \& Vitaro, F. (1997). Boys' behavioral inhibition and the risk of later delinquency. Archives of General Psychiatry, 54, 809-816. 


\section{References}

Kim, K., \& Smith, P. K. (1998). Childhood stress, behavioral symptoms and mother-daughter pubertal development. Journal of Adolescence, 21, 231-240.

Klimstra, T. A., Crocetti, E., Hale, W. W., Kolman, A. I. M, Fortanier, E. L., \& Meeus, W. H.

J. (2009) Identity Formation in Juvenile Delinquents, Clinically Referred Youth, and Adolescents from the General Population. Manuscript submitted for publication.

Klimstra, T. A., Hale III, W. W., Raaijmakers, Q. A. W., Branje, S. J. T., \& Meeus, W. H. J. (2009). Maturation of personality in adolescence. Journal of Personality and Social Psychology, 96, 898-912.

Klimstra, T. A., Hale III, W. W., Raaijmakers, Q. A. W., Branje, S. J. T., \& Meeus, W. H. J. (2010). Identity Formation in Adolescence: Change or Stability? Journal of Youth and Adolescence, 39 , 150-162.

Klimstra, T. A., Luyckx, K., Hale III, W. W., Goossens, L., \& Meeus, W. H. J. (in press). Longitudinal associations between personality profile stability and adjustment in college students: Distinguishing among overall stability, distinctive stability, and within-time normativeness. Journal of Personality.

Kline, R. B. (1998). Principles and practice of structural equation modeling. London: The Guilford Press.

Kovacs, M. (1985). The children's depression, inventory (cdi). Psychopharmacology Bulletin, 21, 995-998.

Kroger, J. (1997). Gender and Identity: The intersection of Structure, Content, and Context. Sex Roles, 36, 747770.

Kroger, J. (2007). Presidential address: The status of identity. Paper presented at the 14th Annual Conference of the Society for Research on Identity Formation, George Washington University, Ashburn, Virginia.

Kroger, J., \& Haslett, S. J. (1988). Separation-individuation and ego identity status in late adolescence: A twoyear longitudinal study. Journal of Youth and Adolescence, 17, 59-79.

Krueger, R. F. (1999). Personality traits in late adolescence predict mental disorders in early adulthood: A prospective-epidemiological study. Journal of Personality, 67, 39-65.

Krueger, R. F., \& Tackett, J. L. (2003). Personality and psychopathology: Working toward the bigger picture. Journal of Personality Disorders, 17, 109-128.

Kunnen, E. S., Bosma, H. A., Van Halen, C. P. M., \& Van der Meulen, M. (2001). A selforganizational approach to identity and emotions: And overview and implications. In H. A. Bosma \& E. S. Kunnen (Eds.), Identity and emotion. Cambridge: University Press.

Laursen, B. (1993). The perceived impact of conflict on adolescent relationships. MerrillPalmer Quarterly, 39, 535-550.

Laursen, B. (1995). Conflict and social interaction in adolescent relationships. Journal of Research on Adolescence, 5, 55-70.

Lehnart, J., \& Neyer, F. J. (2006). Should I stay or should I go?: Attachment and personality in stable and instable romantic relationships. European Journal of Personality, 20, 475-495.

Lichtwarck-Aschoff, A., van Geert, P. L. C., Bosma, H. A., \& Kunnen, E. S. (2008). Time and identity: A framework for research and theory formation. Developmental Review, 28, 370-400.

Little, R. (1988). A test of missing completely at random for multivariate data with missing values. Journal of the American Statistical Association, 83, 1198-1202. 


\section{References}

Little, R. J. A., \& Rubin, D. B. (1987). Statistical analysis with missing data. New York: Wiley.

Lo, Y., Mendell, N. R., \& Rubin, D. B. (2001). Testing the number of components in a normal mixture. Biometrika, 88, 767-778.

Lodi-Smith, J., \& Roberts, B. W. (2007). Social investment and personality: A meta-analysis of the relationship of personality traits to investment in work, family, religion, and volunteerism. Personality and Social Psychology Review, 11, 68-86.

Lönnqvist, J. E.., Mäkinen, S., Paunonen, S. V., Henriksson, M., \& Verkasalo, M. (2008). Psychosocial functioning in young men predicts their personality stability over 15 years. Journal of Research in Personality, 42, 599-621.

Lounsbury, J. W., Steel, R. P., Loveland, J. L., \& Gibson, L. W. (2004). An investigation of personality traits in relation to adolescent school absenteism. Journal of Youth and Adolescence, 33, 457-466.

Lounsbury, J. W., Sunstrom, E., Loveland, J. L., \& Gibson, L. W. (2003). Broad versus narrow personality traits in predicting academic performance in adolescents. Learning and Individual Differences, 14, 67-77.

Luyckx, K., Goossens, L., \& Soenens, B. (2006). A developmental contextual perspective on identity construction in emerging adulthood: Change dynamics in commitment formation and commitment evaluation. Developmental Psychology, 42, 366-380.

Luyckx, K., Goossens, L., Soenens, B., \& Beyers, W. (2006). Unpacking commitment and exploration: Preliminary validation of an integrative model of late adolescent identity formation. Journal of Adolescence, 29, 361-378.

Luyckx, K., Goossens, L., Soenens, B., Beyers, W., \& Vansteenkiste, M. (2005). Identity statuses based upon four rather than two identity dimensions: Extending and refining Marcia's paradigm. Journal of Youth and Adolescence, 34, 605-618.

Luyckx, K., Schwartz, S. J., Berzonsky, M. D., Soenens, B., Vansteenkiste, M., Smits, I., \& Goossens, L. (2008). Capturing ruminative exploration: Extending the four-dimensional model of identity formation in late adolescence. Journal of Research in Personality, 42, 58-82.

Luyckx, K., Schwartz, S. J., Goossens, L., Soenens, B., \& Beyers, W. (2008). Developmental typologies of identity formation and adjustment in female emerging adults: A latent class growth analysis approach Journal of Research on Adolescence, 18, 595-619.

Luyckx, K., Soenens, B., \& Goossens, L. (2006). The personality-identity interplay in emerging adult women: Convergent findings from complementary analyses. European Journal of Personality, 20, 195-215.

Luyckx, K., Soenens, B., Vansteenkiste, M., Goossens, L., \& Berzonsky, M. D. (2007). Parental psychological control and dimensions of identity formation in emerging adulthood. Journal of Family Psychology, 21, 546-550.

Lynn, R. (1994). Sex differences in intelligence and brain size: A paradox resolved. Personality and Individual Differences, 17, 257-271.

Marcia, J. E. (1966). Development and validation of ego-identity status. Journal of Personality and Social Psychology, 3, 551-558.

Marcia, J. E. (1980). Identity in adolescence. In J. Adelson (Ed.), Handbook of adolescent psychology. New York: Wiley.

Marcia, J. E. (1993). The ego identity status approach to ego identity. In J. E. Marcia, A. S. 
Waterman, D. R. Matteson, S. L. Archer, \& J. L. Orlofsky (Eds.), Ego Identity: A Handbook for Psychosocial Research. (pp. 1-21). New York: Springer-Verlag.

Marcia, J. E. (2007). Theory and measure: The identity status interview. In M. Watzlawik \& A. Born (Eds.), Capturing identity: Quantitative and qualitative methods (pp. 1-14). Lanham, MD: University Press of America.

Matteson, D. R. (1977). Exploration and commitment: Sex differences and methodological problems in the use of identity status categories. Journal of Youth and Adolescence, 6, 353-374.

McCrae, R. R. (2008). A note on some measures of profile agreement. Journal of Personality Assessment, 90, 105-109.

McCrae, R. R., \& Costa, P. T. (1987). Validation of the five-factor model of personality across instruments and observers. Journal of Personality and Social Psychology, 52, 81-90.

McCrae, R. R., \& Costa, P. T. (1999). A five-factor theory of personality. In L. Pervin, \& O.

P. John (Eds.), Handbook of personality: Theory and research (2nd ed., pp. 139-153). New York: Guilford Press.

McCrae, R. R., Costa, P. T., Jr., Terracciano, A., Parker, W. D., Mills, C. J., De Fruyt, F., et al. (2002). Personality trait development from age 12 to age 18: Longitudinal, cross-sectional and cross-cultural analyses. Journal of Personality and Social Psychology, 83, 1456-1468.

Meeus, W. H. J. (1996). Studies on identity development in adolescence: An overview of research and some new data. Journal of Youth and Adolescence, 25, 569-598.

Meeus, W. H. J., Akse, J., Branje, S. J. T., Ter Bogt, T. F. M., Crommelin, P. M., Delsing, M. J. M. H., et al. (2006). Codebook of the research project conflict and management of relationships (conamore).Unpublished manuscript, Utrecht University, The Netherlands, 2006.

Meeus, W., Branje, S., van der Valk, I., \& de Wied, M. (2007). Relationships with intimate partner, best friend, and parents in adolescence and early adulthood: A study of the saliency of the intimate partnership. International Journal of Behavioral Development, 31, 449-460.

Meeus, W. H. J., Iedema, J., Helsen, M., \& Vollebergh, W. (1999). Patterns of adolescent identity development: Review of literature and longitudinal analysis. Developmental Review, 19, 419-461.

Meeus, W., Iedema, J., Maassen, G.H. (2002). Commitment and exploration as mechanisms of identity formation. Psychological Reports, 90, 711-785.

Meeus, W. H. J., Iedema, J., Maassen, G., \& Engels, R. (2005). Separation-individuation revisited: On the interplay of parent-adolescent relations, identity and emotional adjustment in adolescence. Journal of Adolescence, 28, 89-106.

Meeus, W., van de Schoot, R., Keijsers, L., Schwartz, S. J., \& Branje, S. (2010). On the Progression and Stability of Adolescent Identity Formation: A Five-Wave Longitudinal Study in Earlyto-middle and Middle-to-late Adolescence. Child Development, 81.

Mehta, P. D., \& West, S. G. (2000). Putting the individual back into individual growth curves. Psychological Methods, 5, 23-43.

Morizot, J., \& Le Blanc, M. (2003). Searching for a developmental typology of personality and its relations to antisocial behaviour: A longitudinal study of an adjudicated men sample. Criminal Behaviour and Mental Health, 13, 241-277. 


\section{References}

Morizot, J., \& Le Blanc, M. (2005). Searching for a developmental typology of personality and its relations to antisocial behavior: A longitudinal study of a representative sample of men. Journal of Personality, 73, 139-182.

Muris, P., Meesters, C., \& Blijlevens, P. (2007). Self-reported reactive and regulative temperament in early adolescence: Relations to internalizing and externalizing problem behavior and 'Big Three' personality factors. Journal of Adolescence, 30, 1035-1049.

Muris, P., Merckelbach, H., Van Brakel, A., \& Mayer, B. (1999). The revised version of the screen for child anxiety related emotional disorders (SCARED-R): Further evidence for its reliability and validity. Anxiety, Stress and Coping, 12, 411-425.

Muris, P., \& Steerneman, P. (2001). The revised version of the screen for child anxiety related emotional disorders (SCARED-R): First evidence for its reliability and validity in a clinical sample. British Journal of Clinical Psychology, 40, 35-44.

Muthén, B. O., \& Muthén, L. K. (2000). Integrating person-centered and variable-centered analyses: Growth mixture modeling with latent trajectory classes. Alcoholism: Clinical and Experimental Research, 24, 882-891.

Muthén, L. K., \& Muthén, B. O. (2007). Mplus user's guide (Fourth ed.). Los Angeles, CA: Muthén \& Muthén.

Muthén, L. K., \& Muthén, B. O. (2007). Mplus user's guide (Fifth ed.). Los Angeles, CA: Muthén \& Muthén.

Nagin, D. S. (1999). Analyzing developmental trajectories: A semi-parametric group-based approach. Psychological Methods, 4, 139-157.

Nagin, D. S. (2005). Group-based Modeling of Development. Cambridge, MA: Harvard University Press.

Neyer, F. J., \& Asendorpf, J. B. (2001). Personality-relationship transaction in young adulthood. Journal of Personality and Social Psychology, 81, 1190-1204.

Nigg, J. T. (2006). Temperament and developmental psychopathology. Journal of Child Psychology and Psychiatry, 47, 395-422

Noftle, E. E., \& Robins, R. W. (2007). Personality predictors of academic outcomes: Big five correlates of gpa and sat scores. Journal of Personality and Social Psychology, 93, 116.

Orth, U., Robins, R. W., \& Roberts, B. W. (2008). Low self-esteem prospectively predicts depression in adolescence and young adulthood. Journal of Personality and Social Psychology, 95, 695-708.

Ozer, D. J., \& Benet-Martínez, V. (2006). Personality and the prediction of consequential outcomes. Annual Review of Psychology, 57, 401-421.

Ozer, D. J., \& Gjerde, P. F. (1989). Patterns of personality consistency and change from childhood through adolescence. Journal of Personality, 57, 483-507.

Paikoff, R. L., \& Brooks-Gunn, J. (1991). Do Parent-Child Relationships Change During Puberty? Psychological Bulletin, 110, 47-66.

Paunonen, S. V. (1998). Hierarchical organization of personality and prediction of behavior. Journal of Personality and Social Psychology, 74, 538-556.

Petersen, A. C., \& Crockett, L. (1985). Pubertal timing and grade effects on adjustment. Journal of Youth and Adolescence, 14, 191-206.

Petersen, A. C., Crockett, L., Richards, M., \& Boxer, A. (1988). A self-report measure of pubertal status reliability, validity, and initial norms. Journal Of Youth And Adolescence, 17, 117-133. 


\section{References}

Phinney, J. S., \& Ong, A. D. (2007). Conceptualization and measurement of ethnic identity:

Current status and future directions. Journal of Counseling Psychology, 54, 271-281.

Pullmann, H., Raudsepp, L., \& Allik, J. (2006). Stability and change in adolescents' personality: A longitudinal study. European Journal of Personality, 20, 447-459.

Prinzie, P., Onghena, P., \& Hellinckx, W. (2005). Parent and child personality traits and children's externalizing problem behavior from age 4 to 9 years: A cohort-sequential latent growth curve analysis. MerrillPalmer Quarterly 51, 335-366.

Radloff, L. S. (1977). The Center for Epidemiologic Studies-Depression Scale: A self-report depression scale for research in the general population. Journal of Applied Psychological Measurement, $1,185-401$.

Roberts, B. W., (1997). Plaster or plasticity: Are adult work experiences associated with personality change in women? Journal of Personality, 65, 205-232.

Roberts, B. W. \& Caspi, A. (2003). The cumulative continuity model of personality development: Striking a balance between continuity and change. In R. M. Staudinger \& U. Lindenberger (Eds.): Understanding Human Development: Life Span Psychology in Exchange with Other Disciplines (pp. 183-214). Dordrecht, The Netherlands: Kluwer Academic.

Roberts, B. W., Caspi, A., \& Moffitt, T. E. (2001). The kids are alright: Growth and stability in personality development from adolescence to adulthood. Journal of Personality and Social Psychology, 81, 670683.

Roberts, B. W., Caspi, A., \& Moffitt, T. E. (2003). Work experiences and personality development in young adulthood. Journal of Personality and Social Psychology, 84, 582-593.

Roberts, B. W., \& DelVecchio, W. F. (2000). The rank-order consistency of personality traits from childhood to old age: A quantitative review of longitudinal studies. Psychological Bulletin, 126, 3-25.

Roberts, B. W., Walton, K. E., \& Viechtbauer, W. (2006). Patterns of mean-level change in personality traits across the life course: A meta-analysis of longitudinal studies. Psychological Bulletin, 132, 1-25.

Roberts, B. W., \& Wood, D. (2006). Personality development in the context of the neo-socioanalytic model of personality. In D. K. Mroczek \& T. D. Little (Eds.), Handbook of personality development. Mahwah, NJ: Lawrence Erlbaum.

Roberts, B. W., Wood, D., \& Smith, J. L. (2005). Evaluating five factor theory and social investment perspectives on personality trait development. Journal of Research in Personality, 39, 166-184.

Robins, R. W., Fraley, R. C., Roberts, B. W., \& Trzesniewski, K. H. (2001). A longitudinal study of personality change in young adulthood. Journal of Personality, 69, 617-640.

Robins, R. W., Hendin, H. M., \& Trzesniewski, K. H. (2001). Measuring Global Self-Esteem: Construct Validation of a Single-Item Measure and the Rosenberg Self-Esteem Scale. Personality and Social Psychology Bulletin, 27, 151-161.

Robins, R. W., John, O. P., Caspi, A., Moffitt, T. E., \& Stouthamer-Loeber, M. (1996). Resilient, overcontrolled, and undercontrolled boys: Three replicable personality types. Journal of Personality and Social Psychology, 70, 157-171. 


\section{References}

Robins, R. W., Noftle, E. E., Trzesniewski, K. H., \& Roberts, B. W. (2005). Do people know how their personality has changed? Correlates of perceived and actual personality change in young adulthood. Journal of Personality, 73, 489-522.

Rosenberg, M. (1965). Society and the adolescent self-image. Princeton, NJ: Princeton University Press.

Ruble, D. N., Martin, C. L., \& Berenbaum, S. (2006). Gender development. In D. Kuhn \& R. Siegler (Eds.), Handbook of child psychology: Cognition, perception and language (6th ed., Vol. 3, pp. 858-932). New York: Wiley.

Salgado, J. F. (1997). The five factor model of personality and job performance in the european community. Journal of Applied Psychology, 82, 30-43.

Satorra, A., \& Bentler, P. M. (1994). Corrections to test statistics and standard errors in covariance structure analysis. In A. Von Eye \& C. C. Clogg (Eds.), Latent variables analysis: Applications for developmental research (pp. 399-419). Thousand Oaks, CA: Sage Publications.

Satorra, A., \& Bentler, P. M. (2001). A scaled difference chi-square test statistic for moment structure analysis. Psychometrika, 66, 507-514.

Schafer, J. L., \& Graham, J. W. (2002). Missing data: Our view of the state of the art. Psychological Methods, 7, $147-177$.

Shaw, D. S., Gilliom, M., Ingoldsby, E. M., \& Nagin, D. S. (2003). Trajectories leading to school-age conduct problems. Developmental Psychology, 39, 189-200.

Scher, S. J., \& Osterman, N. M. (2002). Procrastination, conscientiousness, anxiety, and goals: Exploring the measurement and correlates of procrastination among school-aged children. Psychology in the Schools, 39, 385-398.

Scholte, R. H., van Aken, M. A. G., \& van Lieshout, C. F. M. (1997). Adolescent personality factors in selfratings and peer nominations and their prediction of peer acceptance and peer rejection. Journal of Personality Assessment, 69, 534 - 554.

Schwartz, S. J. (2001). The evolution of eriksonian and neo-eriksonian identity theory and research: A review and integration. Identity, 1, 7-58.

Schwarz, G. (1978). Estimating the dimension of a model. The Annals of Statistics, 6, 461-464.

Selfhout, M., Denissen, J., Branje, S., \& Meeus, W. (2009). In the eye of the beholder: Perceived, actual, and peer-rated similarity in personality, communication, and friendship intensity during the acquaintanceship process. Journal of Personality and Social Psychology, 96, 1152-1165.

Shiner, R., \& Caspi, A. (2003). Personality differences in childhood and adolescence: Measurement, development, and consequences. Journal of Child Psychology and Psychiatry, 44, 2-32.

Shiner, R. L., Masten, A. S., \& Tellegen, A. (2002). A developmental perspective on personality in emerging adulthood: Childhood antecedents and concurrent adaptation. Journal of Personality and Social Psychology, 83, 1165-1177.

Shulman, S., Laursen, B., Kalman, Z., \& Karpovsky, S. (1997). Adolescent intimacy revisited. Journal of Youth and Adolescence, 26, 597-617.

Smits, D. J. M., \& Boeck, P. D. (2006). From BIS/BAS to the Big Five. European Journal of Personality, 20, 255-270.

Soenens, B., Berzonsky, M. D., Vansteenkiste, M., Beyers, W., \& Goossens, L. (2005). 
Identity styles and causality orientations: In search of the motivational underpinnings of the identity exploration process. European Journal of Personality, 19, 427-442.

Soto, C. J., John, O. P., Gosling, S. D., \& Potter, J. (2008). The developmental psychometrics of big five selfreports: Acquiescence, factor structure, coherence, and differentiation from ages 10 to 20. Journal of Personality and Social Psychology, 94, 718-737.

Statistics Netherlands (2003). Jeugd 2003, cijfers en feiten [Dutch youth 2003, numbers and facts]. Voorburg, the Netherlands: Statistics Netherlands.

Statistics Netherlands. (2008a). Statline: Bevolking naar Herkomst en Generatie. Voorburg, The Netherlands: Statististics Netherlands.

Statistics Netherlands. (2008b). Statline: Mbo; leerlingen en geslaagden. Voorburg, The Netherlands: Statististics Netherlands.

Statistics Netherlands. (2008c). Statline: Vo; leerlingen en geslaagden. Voorburg, The Netherlands: Statistics Netherlands.

Statistics Netherlands. (2009a). Statline: Huwen en Partnerschapsregistraties; Kerncijfers. [Statline: Marriage and Partnership Registrations]. Available from: http://www.cbs.nl/nl$\mathrm{NL} / \mathrm{menu} /$ themas/bevolking/cijfers/incidenteel/maatwerk/2388-wm.htm.

Statistics Netherlands. (2009b). Leeftijd Ouders bij Geboorte Kind, 1996 en 2000-2006. [Age of Parents at Birth of Child; 1996 and 2000-2006]. Available from: http://statline.cbs.nl/StatWeb/publication/?VW=T\&DM=SLNL\&PA=37772ned\&D1=a\&D2=0,10,20,30 ,40,50,(1-1)-1\&HD=090812-1411\&HDR=G1\&STB=T.

Steiger, J. H., Shapiro, A., \& Browne, M.W. (1985). On the multivariate asymptotic distribution of sequential chi-square statistics. Psychometrika, 50, 253-264.

Tackett, J. L. (2006). Evaluating models of the personality-psychopathology relationship in children and adolescents. Clinical Psychology Review, 26, 584-599.

Trapnell, P. D., \& Campbell, J. D. (1999). Private Self-Consciousness and the Five-Factor Model of Personality: Distinguishing Rumination From Reflection. Journal of Personality and Social Psychology, 76, 284304.

Twenge, J. M. (2000). The age of anxiety? Birth cohort change in anxiety and neuroticism, 1952-1993. Journal Of Personality And Social Psychology, 79, 1007-1021.

Twenge, J. M. (2001). Birth cohort changes in extraversion: A cross-temporal meta-analysis, 1966-1993. Personality And Individual Differences, 30, 735-748.

van Aken, M. A. G., \& Dubas, J.-S. (2004). Personality type, social relationships, and problem behaviour in adolescence. European Journal of Developmental Psychology, 1, 331-348.

van Aken, M. A. G., Van Lieshout, C. F. M., Scholte, R. H. J., \& Haselager, G. J. T. (2002). Personality types in childhood and adolescence: Main effects and person-relationship transactions. In L. Pulkkinen \& A. Caspi (Eds.), Paths to successful development: Personality in the life course. Cambridge: University Press.

Vandenberg, R. J., \& Lance, C. E. (2000). A review and synthesis of the measurement invariance literature: Suggestions, practices, and recommendations for organizational research. Organizational Research Methods, 3, 4-70. 


\section{References}

van Doorn, M. D., Branje, S. J. T., \& Meeus, W. H. J. (2008). Conflict resolution in parent-adolescent relationships and adolescent delinquency. Journal of Early Adolescence, 28, 503-527.

van Hoof, A. (1999). The identity status field re-reviewed: An update of unresolved and neglected issues with a view on some alternative approaches. Developmental Review, 19, 497-55

Van Leeuwen, K. G., Mervielde, I., Braet, C., \& Bosmans, G. (2004). Child personality and parental behavior as moderators of problem behavior: Variable- and person-centered approaches. Developmental Psychology, 40, 1028-1046.

Van Leeuwen, K., Mervielde, I., De Clercq, B., \& De Fruyt, F. (2007). Extending the spectrum idea: Child personality, parenting and psychopathology. European Journal of Personality, 21, 63-89.

van Lieshout, C. F. M. (2000). Lifespan personality development: Self-organising goal-oriented agents and developmental outcome. International Journal of Behavioral Development, 24, 276-288.

Vollebergh, W. A. M., Iedema, J., \& Raaijmakers, Q. A. W. (2001). Intergenerational transmission and the formation of cultural orientations in adolescence and young adulthood. Journal Of Marriage And The Family, 63, 1185-1198.

Von Bertalanffy, L. (1968). General System Theory. New York: George Braziller, Inc.

Warner, M. B., Morey, L. C., Finch, J. F., Gunderson, J. G., Skodol, A. E., Sanislow, C. A., et al. (2004). The longitudinal relationship of personality traits and disorders. Journal of Abnormal Psychology, 113, 217-227.

Waterman, A. S. (1982). Identity development from adolescence to adulthood: An extension of theory and a review of research. Developmental Psychology, 18, 342-358.

Waterman, A. S. (1993). Developmental perspectives on identity formation: From adolescence to adulthood. In J. E. Marcia, A. S. Waterman, D. R. Matteson, S. Archer \& J. L. Orlofsky (Eds.), Ego identity. New York: Springer Verlag.

Waterman, A. S. (1999). Identity, the identity statuses, and identity status development: A contemporary statement. Developmental Review, 19, 591-621.

Weinmann, L. L. (1992). Patterns of change in middle adolescent adjustment: The role of relationships with parents and peers. Dissertation Abstracts International, 53, 2565B-2566B. (University Microfilms No. DA92-18128).

Widiger, T. A., \& Trull, T. J. (1992). Personality and psychopathology: An application of the five-factor model. Journal of Personality, 60, 363-393.

Widiger, T. A., Verheul, R., \& van den Brink, W. (1999). Personality and psychopathology. In L. A. Pervin \& O. P. Johns (Eds.), Handbook of Personality: Theory and Research. New York: Guilford.

Willett, J .B., Singer, J. D., Martin, N. C. (1998). The design and analysis of longitudinal studies of development and psychopathology in context: Statistical models and methodological recommendations. Development and Psychopathology, 10, 395-426. 


\section{Summary}

The current dissertation focused on adolescent identity formation and personality development. We focused on how these aspects of individuality develop through adolescence, and how individual differences in (the development of) personality and identity relate to problem behavior. For that purpose, we applied: a) variable-centered approaches focusing on how particular traits or dimensions change on average in a group of individuals, and whether individual differences in traits or dimensions are stable or fluctuate across time; and b) person-centered approaches focusing on how a configuration of traits is organized within an individual.

Data were used from three longitudinal projects: CONAMORE, RADAR, and LTIDES. In addition, data from a cross-sectional project involving juvenile delinquents and clinically referred youth was used. Participants in these projects filled out questionnaires on personality, identity formation, conflicts with parents, friendship quality, academic adjustment, and problem behavior.

Variable-centered approaches to personality revealed that adolescent's personalities matured, as they became more agreeable, extraverted, and open to experience when they grew older. In addition, inter-individual differences became more set, and traits became more consistently organized within individuals. For all aspects of development, girls were ahead on boys. Development of personality traits was strongly interrelated with development of problem behavior symptoms, as specific personality characteristics seemed to put adolescents at risk for developing problem behavior symptoms, but problem behavior symptoms also seemed to affect adolescent personality traits.

Person-centered approaches to personality revealed that three classic personality types (Resilients, Undercontrollers, and Overcontrollers) were replicable as trajectories of personality development. With another person-centered approach to personality, we showed that it is not the stability of a profile that is linked to psychological adjustment, but the degree to which an individual's personality profile matches the profile of the average person within a sample. From a developmental perspective, an individual's personality profile can diverge from the profile of the average person in two ways: one can either be ahead (i.e., hypermaturity) or lag behind (i.e., immaturity) in development. We showed that hypermaturity is related to internalizing problems in boys and girls. Immaturity is related to externalizing problems, but only in girls.

With regard to identity formation, early adolescents became increasingly more certain about their current commitments as they exhibited decreases in reconsideration. Middle and 
Summary

late adolescents were involved in identity evaluation, as they exhibited increases in in-depth exploration. As with personality development, girls also seemed to be ahead on boys in identity formation. By tracking early adolescents with daily internet assessments, we demonstrated that identity formation is guided by a certainty-uncertainty (commitmentreconsideration) dynamic that operates on day-to-day level. Moreover, fluctuations in reconsideration predicted a weaker sense of identity in general. Finally, we demonstrated that identity formation is more strongly related to severe forms of delinquency than to other types of severe problem behavior, as juvenile delinquents displayed much more problems with identity formation than clinically referred youth did.

In sum, we demonstrated important development changes in personality and identity. Individual differences in (changes in) personality and identity were found to be strongly related to problem behavior. 


\section{Samenvatting}

In deze dissertatie werden identiteits- en persoonlijkheidsontwikkeling tijdens de adolescentie onderzocht. We hebben hierbij onderzocht hoe deze aspecten van individualiteit zich ontwikkelden in de adolescentie en hoe inter-individuele verschillen in (de ontwikkeling van) persoonlijkheid en identiteit samenhingen met probleemgedrag. Hiervoor hebben we gebruik gemaakt van: a) variabele-gerichte benaderingen die zich richten op veranderingen in trekken of dimensies op groepsniveau, en stabiliteit of fluctuaties in inter-individuele verschillen in trekken of dimensies; en b) persoonsgerichte benaderingen die zich richten op de wijze waarop een configuratie van trekken of dimensies georganiseerd is binnen een persoon.

Er is gebruik gemaakt van data uit drie longitudinale projecten: CONAMORE, RADAR, en L-TIDES. Daarnaast hebben we gebruik gemaakt van cross-sectionele data over delinquente jongeren en jongeren uit een klinische instelling. De deelnemers aan deze projecten hebben vragenlijsten ingevuld over persoonlijkheid, identiteit, conflicten met ouders, vriendschapkwaliteit, school, en probleemgedrag.

Met behulp van variabele-gerichte benaderingen toonden we aan dat jongeren een meer volwassen persoonlijkheid ontwikkelen, aangezien ze vriendelijker, meer extravert en meer open voor nieuwe ideeën werden naarmate ze ouder werden. Bovendien kwamen interindividuele verschillen in persoonlijkheidstrekken en het persoonlijkheidprofiel van jongeren steeds meer vast te liggen. Voor al deze aspecten van ontwikkeling leken meisjes voor te liggen op jongens. De ontwikkeling van persoonlijkheidstrekken was sterk gerelateerd aan probleemgedrag, aangezien jongeren met specifieke persoonlijkheidstrekken meer risico liepen op het ontwikkelen van probleemgedrag, terwijl probleemgedrag ook leidde tot veranderingen in persoonlijkheidstrekken.

Met persoonsgerichte benaderingen toonden we ten eerste aan dat de drie klassieke persoonlijkheidtypes (Resilients, Undercontrollers en Overcontrollers) gerepliceerd kunnen worden als ontwikkelingstrajecten van persoonlijkheid. Met een tweede persoonsgerichte studie toonden we aan dat niet de stabiliteit van persoonlijkheidsprofielen, maar de mate waarin het persoonlijkheidsprofiel van een individu lijkt op het persoonlijkheidprofiel van een gemiddeld persoon, gerelateerd is aan probleemgedrag. Vanuit een ontwikkelingsperspectief kan een persoonlijkheidsprofiel van een individu op twee manieren afwijken van het gemiddelde: het kan er op wijzen dat een persoon in zijn of haar ontwikkeling voorligt (te volwassen) of achterligt (onvolwassen). Wij hebben aangetoond dat een te volwassen persoonlijkheidsprofiel samenhangt met internaliserende problemen in jongens en meisjes. 


\section{Samenvatting}

Een onvolwassen persoonlijkheid was gerelateerd aan externalizerende problemen bij meisjes, maar niet bij jongens.

Met betrekking tot identiteitsontwikkeling werden vroege adolescenten steeds zekerder van hun huidige bindingen, aangezien ze deze steeds minder gingen heroverwegen. Midden tot late adolescenten hielden zich in toenemende mate bezig met identiteitsevaluatie processen, wat bleek uit een toename van exploratie van de huidige bindingen. Net zoals bij persoonlijkheidsontwikkeling, leken meisjes ook iets voor te liggen in de identiteitsontwikkeling. Hiernaast konden we, door vroege adolescenten te volgen met internetmetingen, aantonen dat identiteitsontwikkeling verloopt aan de hand van een dynamisch proces van zekerheids tegenover onzekerheid (ofwel binding tegenover heroverweging). Bovendien leiden fluctuaties in het niveau van heroverweging tot een zwakkere identiteit in het algemeen. Als laatste toonden we aan dat identiteitsontwikkeling sterker lijkt te samenhangen met delinquentie dan met andere zware vormen van probleemgedrag, aangezien delinquente jongeren meer problemen met identiteitsontwikkeling ondervonden dan jongeren uit een klinische instelling.

Samengevat hebben we aangetoond dat de identiteit en persoonlijkheid van jongeren zich sterk ontwikkelen tijdens de adolescentie. Individuele verschillen in (veranderingen van) persoonlijkheid en identiteit zijn sterk gerelateerd aan probleemgedrag. 
Dit proefschrift was zonder de inbreng van een aantal mensen nooit tot stand gekomen. Deze mensen wil ik hier graag bedanken.

Allereerst wil ik mijn promotor en co-promotoren bedanken. Wim, zonder je scherpe, maar stimulerende feedback en de fantastische datasets waarmee je me liet werken was dit proefschrift nooit in deze vorm tot stand gekomen. Hierbij heb je bovendien veel vrijheid gegeven om mijn eigen ideeën uit te werken, ook als je het niet helemaal met die ideeën (normatieve ontwikkeling en persoonlijkheidstypes) eens was. Daarnaast vond ik het erg leuk om af en toe even over wielrennen te kunnen praten met iemand die er ook echt verstand van heeft. Bill, voordat ik begon als aio dacht ik dat ik al aardig kon schrijven, maar jij hebt me echt wetenschappelijk leren schrijven. Als ik weer eens in statistisch gewauwel bleef hangen, bleef jij net zo lang "what do you mean" bij mijn teksten zetten tot het er in normale bewoordingen stond. Bovendien heb je me gestimuleerd om zo veel mogelijk verschillende ervaringen op te doen, zowel op het gebied van onderzoek als onderwijs. Iets wat ik ook erg waardeer is dat je in het afgelopen jaar af en toe de rollen omdraaide en mij feedback op jou papers liet geven. Daarnaast kon ik ook altijd bij je terecht als me iets dwars zat. Thanks, man! Quinten, we hebben misschien niet heel veel hoeven te overleggen, maar jou inbreng was cruciaal. Als we compleet vast liepen met de statistiek had jij altijd weer een simpele maar doeltreffende oplossing. Vooral het feit dat je complexe zaken op zo'n simpele manier uit kunt leggen maakt samenwerking met jou geweldig!

Tijdens mij aio-schap heb ik ook het geluk gehad om drie maanden in Leuven door te kunnen brengen. Luc, bedankt voor je inzit om dit allemaal geregeld te krijgen. Je was een geweldige gastheer. De twee weken bij jou de kamer waren bijzonder gezellig, ondanks dat je me zeker drie keer per ongeluk op hebt gesloten. Koen, de samenwerking met jou was echt super! Je was altijd bereid om even te overleggen en daardoor hebben we in drie maanden tijd de basis kunnen leggen voor twee van mijn beste papers. Hopelijk kan ik vanaf dit najaar voor langere tijd naar Leuven komen, zodat we deze goede samenwerking verder uit kunnen bouwen.

Dan zijn er mijn co-auteurs. Ook jullie hebben een enorme bijdrage geleverd aan dit proefschrift. Ik wil er twee in het bijzonder noemen. Susan, ik heb me altijd verbaasd over hoe je naast de dagelijkse begeleiding van 4 aio's, een hoop onderwijs en nog allemaal andere dingen toch nog tijd kon vinden om ook uitgebreid feedback te kunnen geven op drie van mijn papers. Bedankt hiervoor! Elisabetta, it has been a great pleasure working with you! 
During your visits to the Netherlands we were able to exchange a lot of idea's which has been a very stimulating experience for me. Hopefully, we can continue our collaboration in the future!

Minstens zo belangrijk als co-auteurs, promotors en co-promotors zijn natuurlijk alle collega's van pedagogiek! Ik wil toch weer een paar mensen in het bijzonder noemen. Sophie en Karlijn, jullie waren echt super-roomies! Met jullie kon ik ook altijd alles bespreken, bedankt daarvoor! Ik heb jullie natuurlijk ook niet voor niks als paranimfen gekozen, want met wie kun je beter je promotie afwachten in het "zweetkamertje" dan met de mensen met wie je al bijna 4 jaar in een naar archief ruikende kamer zit ;-).

Natuurlijk wil ik ook Amaranta bedanken! Dankzij jou (en in eerste instantie ook dankzij Hanneke) is de aio-vrijdagmiddag borrel ingevoerd en het maakt niet uit dat ik de enige lijk te zijn die daarvan op de hoogte is ;-). Zonder jou was het de afgelopen jaren echt stukken minder gezellig geweest!

Ook wil ik mijn mede Gutenberg stamgasten Tom, Loes en Maarten bedanken. Behalve het altijd gezellige koffie drinken hebben we de afgelopen jaren verder ook echt allemaal superleuke dingen kunnen doen. Zo ben ik er achter gekomen dat Southpark en The Simpsons kijken toch leuker is met een beamer van IDC. Loes, de culturele uitwisselingen met jou waren ook erg leuk: jij mee te wadlopen, ik mee naar carnaval. Verder waren er natuurlijk de vakanties die we aan de congressen vast plakten! Chicago met Jerry Springer, Sponge Bob 4D, The Pogues, NOFX en Tom's burning sensations... Later onze roadtrip door Colorado met Amaranta! Volgens mij is het echt zeldzaam dat je collega's treft met wie je zo relaxed zoveel leuke dingen kunt doen! Jullie zijn echt super-collega's!

Dan wol ik no noch wat Fryzen bedanke. Allerearst Freerk en Folkert. Ik kin jim al jieren (folgens myn berekeningen 23 en 16 jier) en jim binne altiid hiele goeie freonen west! Zelfs no we toch wol in stuk fjierder van elkoar wenje hoarde we gelukkig noch regelmatig kontakt. Fan de letste jieren wie foaral de Amstel Gold Race (en foaral de jûne der foar) in hichtepunt! Fjierder fiin ik it erg leuk dat jim ek echt snappe wer ik sa ongevear my bezig bin!

As letste wol ik natuurliik Mem en Rennie bedanke! Foaral toen ik krekt wy begûn, sieten we yn in minne tiid. Troch dat ik der my jim altiid goed oer praatte koe, bin ik der goed trochhinne kam en haw ik de ôfronne jirren kinne dwaan wat ik dien ha: dit proefskrift skriuwe. Derom is dit proefskrift ek foaral foar jim. Dankewol. 


\section{Curriculum Vitae}

Theo Klimstra was born on the $8^{\text {th }}$ of July 1982 in Harlingen, the Netherlands. He completed Senior General Secondary Education (HAVO) at the Dockinga College in Dokkum in 1999, and completed University Preparatory Education (VWO) at the same school in 2001. From 2001 to 2005 he attended the University of Groningen and earned a Master's degree in Psychology. From 2005 to 2006 he worked as a research assistant at the TRAILS-CC project at Accare Groningen. In 2006 he started his PhD-project at the Research Centre Adolescent Development of Utrecht University. From September 2006 to February 2010 he worked on his dissertation on adolescent personality development and identity formation and presented his work at several national and international conferences. During this period of three years and six months, he wrote eight papers and a book chapter as a first author, and co-authored four other papers. He also supervised bachelor- and mastertheses, and worked as a workgroup teacher in an undergraduate course. In 2008, he stayed in Leuven, Belgium for three months for a work visit to Koen Luyckx and Luc Goossens at the Catholic University Leuven. In 2010, he was involved in teaching at and the organization of an intensive two-week Erasmus course on identity formation in the context of globalization in Vilnius, Lithuania, for undergraduate students from Italy, Lithuania, Portugal, Turkey, and the Netherlands. 


\section{Publications}

\section{This dissertation:}

Klimstra, T. A., Akse, J., Hale, W. W., Raaijmakers, Q. A. W., \& Meeus, W. H. J. (in press). Longitudinal associations between Big Five personality traits and problem behavior in adolescence. Journal of Research in Personality.

Klimstra, T. A., Crocetti, E., Hale, W. W., Kolman, A. I. M, Fortanier, E. L., \& Meeus, W. H. J. (2009). ). Identity formation in juvenile delinquents, clinically referred youth, and adolescents from the general population. Manuscript submitted for publication

Klimstra, T. A., Hale, W. W., Raaijmakers, Q. A. W., Branje, S. J. T., \& Meeus, W. H. J. (2009). Maturation of personality in adolescence. Journal of Personality and Social Psychology, 96, 898-912.

Klimstra, T. A., Hale, W. W., Raaijmakers, Q. A. W., Branje, S. J. T., \& Meeus, W. H. J. (2010). Identity formation in adolescence: Change or stability? Journal of Youth and Adolescence, 39, 150-162.

Klimstra, T. A., Hale, W. W., Raaijmakers, Q. A. W., \& Meeus, W. H. J. (2010). Too early or too late: Hypermaturity and immaturity of personality in adolescent boys and girls. Manuscript submitted for publication.

Klimstra, T. A., Hale, W. W., Raaijmakers, Q. A. W., Branje, S. J. T., \& Meeus, W. H. J. (in press). A developmental typology of adolescent personality. European Journal of Personality.

Klimstra, T. A., Hale, W. W., Raaijmakers, Q. A. W., Branje, S. J. T., \& Meeus, W. H. J. (in press). Personality assessment and maturation. In R. J. R. Levesque (Ed.), Encyclopedia of adolescence. Heidelberg, Germany: Springer-Verlag.

Klimstra, T. A., Luyckx, K., Hale, W. W., Frijns, T., Van Lier, P. A. C., \& Meeus, W. H. J. (in press). Short-term fluctuations in identity: Introducing a micro-level approach to identity formation. Journal of Personality and Social Psychology.

Klimstra, T. A., Luyckx, K., Hale, W. W., Goossens, L., \& Meeus, W. H. J. (in press). Longitudinal associations between personality profile stability and adjustment in college students: Distinguishing among overall stability, distinctive stability, and within-time normativeness. Journal of Personality. 


\section{Other publications:}

Crocetti, E., Schwartz, S. J., Fermani, A., Klimstra, T., \& Meeus, W. (2010). A cross-cultural comparison of identity statuses between two European countries. Manuscript submitted for publication.

Crocetti, E., Klimstra, T., Keijsers, L., Hale, W. W., \& Meeus, W. (2009). Anxiety trajectory classes and identity development in adolescence: A five-wave longitudinal study. Journal of Youth and Adolescence, 38, 839-849.

Hale, W. W., Klimstra, T. A., \& Meeus, W. H. J. (in press). Is the generalized anxiety disorder symptom of worry just another form of neuroticism?: A five-year longitudinal study of adolescents from the general population. Journal of Clinical Psychiatry.

Hale, W. W., Klimstra, T. A., Wijsbroek, S. A. M., Raaijmakers, Q. A. W., Muris, P., Van Hoof, A., \& Meeus, W. H. J. (2009). Ontwikkelingstrajecten van angstsymptomen: Een vijfjarig prospectief onderzoek onder adolescenten uit de algemene populatie. Tijdschrift voor Psychiatrie, 51, 21-30. 